\title{
Flow Electrosynthesis of Sulfoxides, Sulfones and Sulfoximines without Supporting Electrolytes
}

\author{
Nasser Amri and Thomas Wirth* \\ School of Chemistry, Cardiff University, Main Building, Park Place, Cardiff CF10 3AT, U.K. \\ Email: wirth@cf.ac.uk
}

Supporting Information 


\section{Table of Contents}

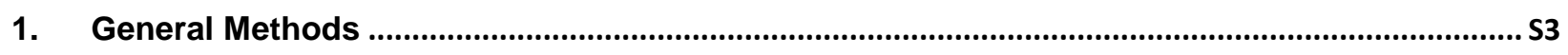

2. Automated flow and electrochemical reactor setup ................................................................. 4

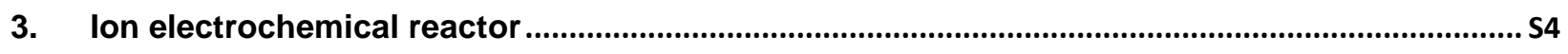

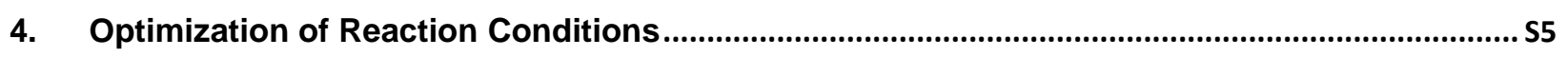

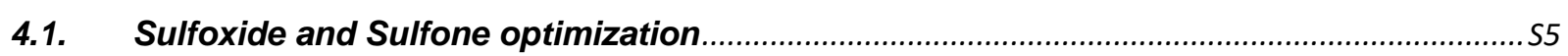

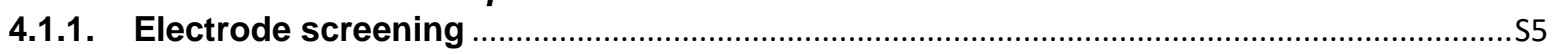

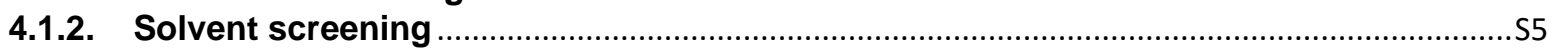

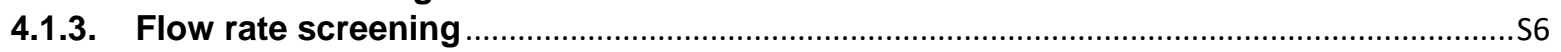

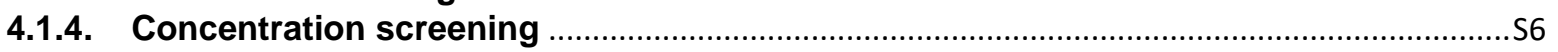

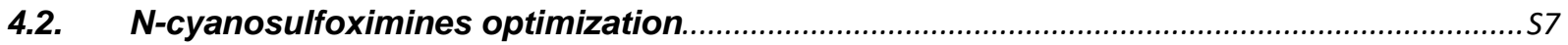

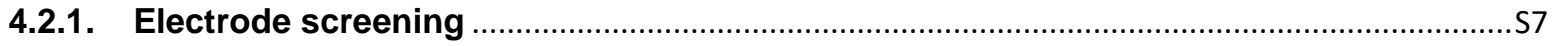

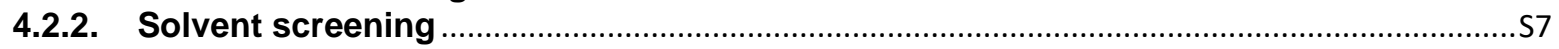

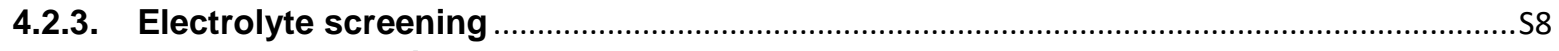

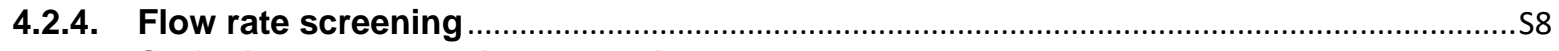

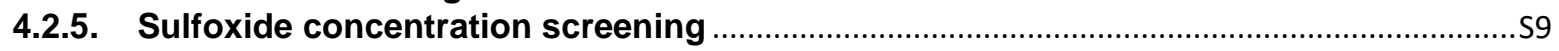

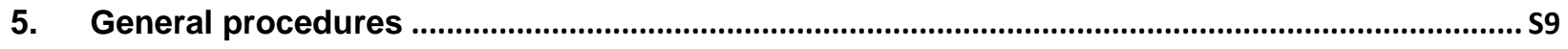

5.1. General procedure for the electrochemical oxidation of sulfides to sulfoxides (GP1):....S9

5.2. General procedure for the electrochemical oxidation of sulfides to sulfones (GP2):.......S9

5.3. General procedure for the electrochemical imination of sulfoxides (GP3):.....................S10

5.4. General procedure for the reduction of $\mathrm{N}$-cyanosulfoximine (GP4): :...............................S10

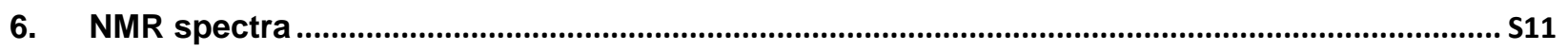




\section{General Methods}

All solvents and reagents were used as received without purification or drying. Thin-layer chromatography (TLC) was performed on pre-coated aluminium sheets of Merck silica gel 60 F254 $(0.20 \mathrm{~mm})$ and visualized by UV radiation $(254 \mathrm{~nm})$. Automated column chromatography was performed on a Biotage ${ }^{\circledR}$ Isolera Four using Biotage ${ }^{\circledR}$ cartridges SNAP Ultra 10 g. ${ }^{1} \mathrm{H}$ NMR, ${ }^{13} \mathrm{C}$ NMR and ${ }^{19} \mathrm{~F}$ NMR spectra were measured on Bruker DPX 400 or 500 apparatus and were referenced to the residual proton solvent peak ( ${ }^{1} \mathrm{H}: \mathrm{CDCl}_{3}, \delta 7.26 \mathrm{ppm}$; DMSO- $\left.\mathrm{d}_{6}, \delta 2.50 \mathrm{ppm}\right)$ and solvent ${ }^{13} \mathrm{C}$ signal $\left(\mathrm{CDCl}_{3}, \delta 77.2 \mathrm{ppm}\right.$, DMSO- $\left.\mathrm{d}_{6}, \delta 39.5\right)$. Chemical shifts $\delta$ were reported in ppm, multiplicity of the signals was declared as followed: $s=$ singlet, $d=$ doublet, $t=$ triplet, $q=$ quartet, quin = quintet, sex = sextet, hep $=$ septet, $d d=$ doublet of doublets, $m=$ multiplet, $b=$ broad; and coupling constants (J) in Hertz. IR spectra were recorded on a Shimadzu FTIR Affinity$1 S$ apparatus. Wavenumbers are quoted in $\mathrm{cm}^{-1}$. All compounds were measured neat directly on the crystal of the IR machine. Mass spectrometric measurements were performed by R. Jenkins, R. Hick, T. Williams and S. Waller at Cardiff University on a Water LCR Premier XEtof. lons were generated by Electron Ionisation (EI) and Electron Spray (ES). The molecular ion peaks values quoted for either molecular ion $[\mathrm{M}]^{+}$, molecular ion plus hydrogen $[\mathrm{M}+\mathrm{H}]^{+}$or molecular ion plus sodium $[\mathrm{M}+\mathrm{Na}]^{+}$. Melting points were measured using a Gallenkamp variable heater with samples in open capillary tubes. The electrochemical reactions were carried out in a galvanostatic mode using a Vapourtec lon Electrochemical flow reactor powered up by an lon electrochemical power supply. The cyclic voltammogram studies were performed using an Orygalys OGF500 Potentiostat / Galvanostat with OGFPWR power supply. 


\section{Automated flow and electrochemical reactor setup}

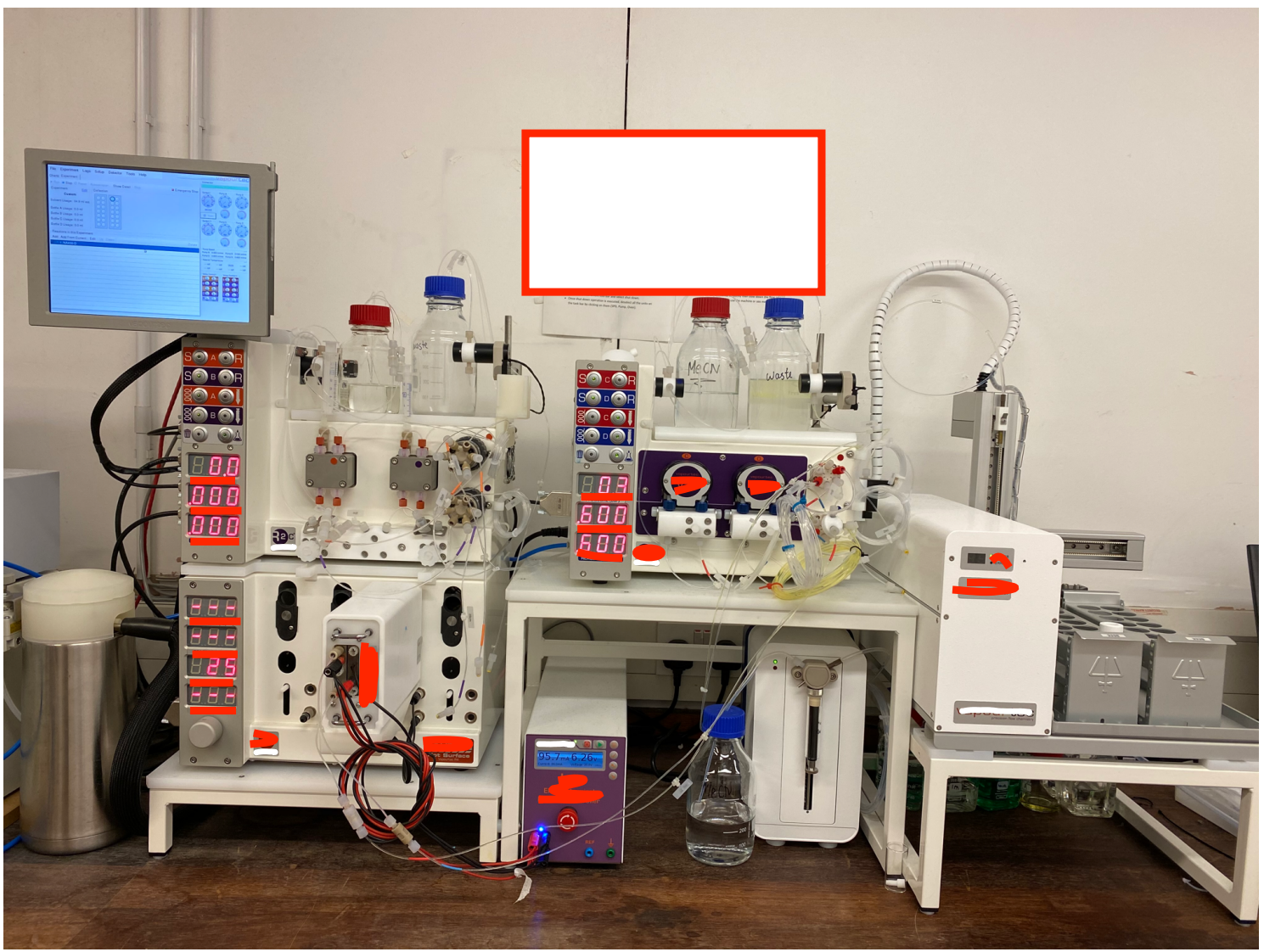

Figure S1. Actual flow set-up of the Ion electrochemical reactor combined with an $\mathrm{R}^{4}$-Series Vapourtec system. (picture: N. Amri)

3. Ion electrochemical reactor

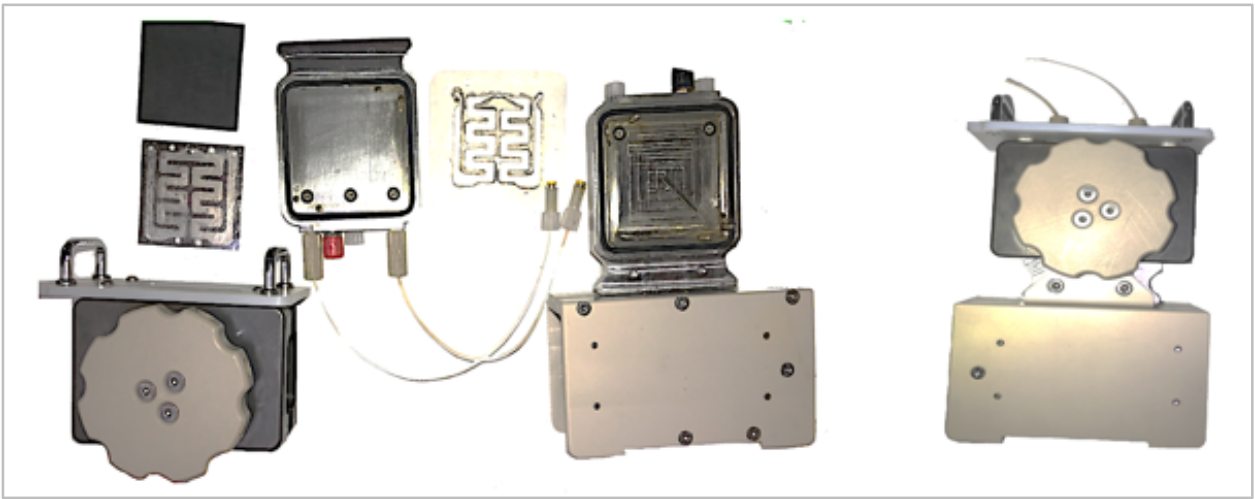

Figure S2. Ion electrochemical reactor. (picture: N. Amri) 


\section{Optimization of Reaction Conditions}

\subsection{Sulfoxide and Sulfone optimization}

\subsubsection{Electrode screening}

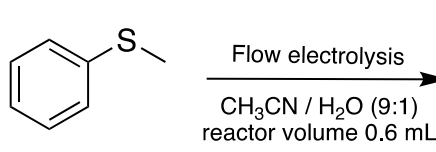

$1 a$<smiles>CS(=O)c1ccccc1</smiles>

$2 a$

$3 a$

\begin{tabular}{|c|c|c|c|c|c|c|c|}
\hline Entry & Anode & Cathode & $\begin{array}{l}\text { Flow rate } \\
{\left[\mathrm{mL} \mathrm{min}^{-1}\right]}\end{array}$ & $\begin{array}{c}\text { Current } \\
\text { [mA] }\end{array}$ & Conversion (\%) & $\% \mathbf{2} \mathbf{a}^{\mathbf{a}}$ & $\% 3 a^{a}$ \\
\hline 1 & $\mathbf{P t}$ & $\mathbf{P t}$ & 0.2 & 64 & 90 & 83 & 7 \\
\hline 2 & Pt & Pt on $\mathrm{Ti}$ & 0.2 & 64 & 95 & 92 & 3 \\
\hline 3 & $\mathbf{P t}$ & $\mathrm{Gr}$ & 0.2 & 64 & 98 & 86 & 12 \\
\hline 4 & Pt on $\mathrm{Ti}$ & $\mathbf{P t}$ & 0.2 & 64 & 90 & 90 & 0 \\
\hline 5 & Gr & Gr & 0.2 & 64 & 96 & 96 & 0 \\
\hline 6 & Gr & Pt & 0.2 & 64 & 98 & 98 & 0 \\
\hline 7 & $\mathbf{G r}$ & $\mathrm{Fe}$ & 0.2 & 64 & 98 & 98 & 0 \\
\hline 8 & GC & $\mathbf{P t}$ & 0.2 & 64 & 98 & 98 & 0 \\
\hline 9 & $\mathbf{F e}$ & $\mathrm{Fe}$ & 0.2 & 64 & 48 & 48 & 0 \\
\hline 10 & Gr & $\mathrm{Fe}$ & 0.2 & 128 & 100 & 27 & 73 \\
\hline 11 & Pt & $\mathrm{Fe}$ & 0.2 & 128 & 98 & 40 & 58 \\
\hline 12 & Pt & $\mathrm{Fe}$ & 0.2 & 160 & 98 & 11 & 87 \\
\hline 13 & Pt & $\mathrm{Fe}$ & 0.2 & 192 & 99 & 12 & 87 \\
\hline
\end{tabular}

Table S1. ${ }^{a}$ The yield determined using ${ }^{1} \mathrm{H}$ NMR with 1,3,5-trimethoxybenzene as internal standard

\subsubsection{Solvent screening}

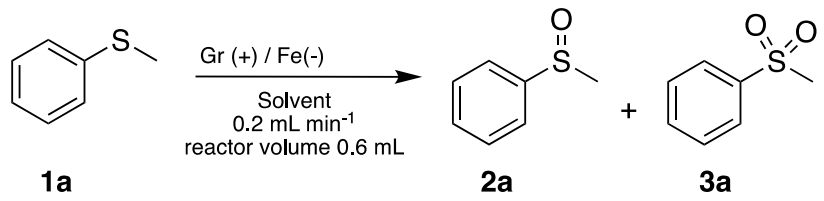

\begin{tabular}{|c|c|c|c|c|c|}
\hline Entry & Solvent & Current [mA] & Conversion (\%) & $\% 2 a^{a}$ & $\% 3 a^{a}$ \\
\hline 1 & $\mathrm{MeCN} / \mathrm{H}_{2} \mathrm{O}(9: 1)$ & 64 & 98 & 98 & 0 \\
\hline 2 & $\mathrm{MeOH} / \mathrm{H}_{2} \mathrm{O}(9: 1)$ & 64 & 95 & 95 & 0 \\
\hline 3 & $\begin{array}{c}\mathrm{MeCN} / \mathrm{MeOH} / \mathrm{H}_{2} \mathrm{O} \\
(8: 1: 1)\end{array}$ & 64 & 88 & 88 & 0 \\
\hline 4 & $\begin{array}{c}\mathrm{MeCN} / \mathrm{HFIP} / \mathrm{H}_{2} \mathrm{O} \\
(8: 1: 1)\end{array}$ & 64 & 93 & 84 & 9 \\
\hline 5 & $\begin{array}{c}\mathrm{MeCN} / \mathrm{MeOH} / \mathrm{H}_{2} \mathrm{O} \\
(4.5: 4.5: 1)\end{array}$ & 64 & 88 & 88 & 0 \\
\hline
\end{tabular}




\subsubsection{Flow rate screening}

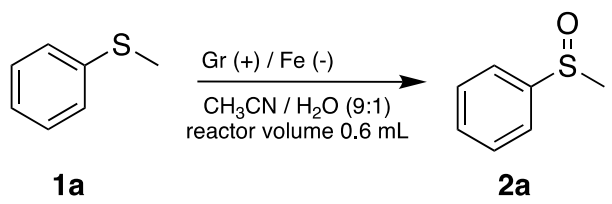

\begin{tabular}{ccccc} 
Entry & Flow rate $\left[\mathbf{m L} \mathbf{~ m i n}^{-1}\right]$ & Current $[\mathbf{m A}]$ & Residence time (min) & $\% \mathbf{~ 2}^{\mathbf{a}}$ \\
\hline 1 & 0.1 & 32 & 6 & 98 \\
2 & 0.15 & 48 & 4 & 98 \\
3 & 0.2 & 64 & 3 & 98 \\
4 & 0.25 & 80 & 2.4 & 92 \\
5 & 0.3 & 96 & 2 & 89
\end{tabular}

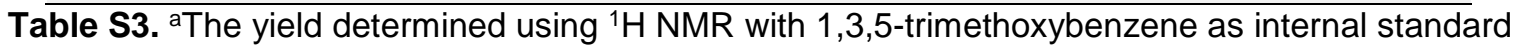

\subsubsection{Concentration screening}<smiles>CSc1ccccc1</smiles>

$1 a$

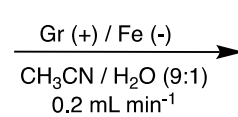

reactor volume $0.6 \mathrm{~mL}$<smiles>CS(=O)c1ccccc1</smiles>

2a<smiles>CS(=O)(=O)c1ccccc1</smiles>

$3 a$

\begin{tabular}{cccccc} 
Entry & $\begin{array}{c}\text { Concentration } \\
(\mathbf{M})\end{array}$ & $\begin{array}{c}\text { Current } \\
{[\mathbf{m A}]}\end{array}$ & Conversion (\%) & $\mathbf{\%} \mathbf{2}^{\mathbf{a}}$ & $\% \mathbf{3}^{\mathbf{a}}$ \\
\hline 1 & 0.1 & 64 & 98 & $>95$ & 0 \\
2 & 0.15 & 96 & 95 & 89 & 6 \\
3 & 0.2 & 128 & 94 & 88 & 6 \\
\hline
\end{tabular}

Table S4. ${ }^{2}$ The yield determined using ${ }^{1} \mathrm{H}$ NMR with 1,3,5-trimethoxybenzene as internal standard 


\section{2. $N$-cyanosulfoximines optimization}

\subsubsection{Electrode screening}

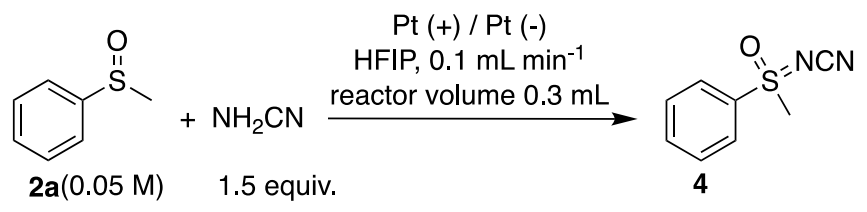

\begin{tabular}{cccc} 
Entry & Anode/Cathode & Current (mA) & Conversion (\%) \\
\hline $\mathbf{1}$ & $\mathrm{C} / \mathrm{Pt}$ & $24(3 \mathrm{~F})$ & $\mathrm{NR}$ \\
$\mathbf{2}$ & $\mathrm{C} / \mathrm{C}$ & $24(3 \mathrm{~F})$ & $\mathrm{NR}$ \\
$\mathbf{3}$ & $\mathrm{C} / \mathrm{Fe}$ & $24(3 \mathrm{~F})$ & $\mathrm{NR}$ \\
$\mathbf{4}$ & $\mathrm{Pt} / \mathrm{C}$ & $24(3 \mathrm{~F})$ & 8 \\
$\mathbf{5}$ & $\mathrm{Pt} / \mathrm{Pt}$ & $24(3 \mathrm{~F})$ & 25 \\
\hline
\end{tabular}

Table S5. The conversion determined using ${ }^{1} \mathrm{H}$ NMR spectroscopy

$\mathrm{NR}=$ No reaction $=$ Starting materials recovered

\subsubsection{Solvent screening}

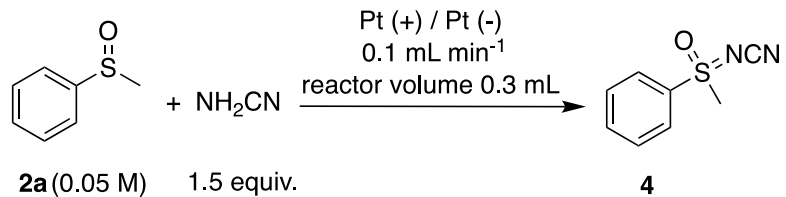

\begin{tabular}{ccccc} 
Entry & Solvent & Current $(\mathbf{m A})$ & Electrolyte & Conversion (\%) \\
\hline $\mathbf{1}$ & $\mathrm{MeCN}$ & $24(3 \mathrm{~F})$ & $\mathrm{Et}_{4} \mathrm{NCl}$ & 18 \\
$\mathbf{2}$ & $\mathrm{MeCN}_{\mathbf{3}}$ & $40(5 \mathrm{~F})$ & $\mathrm{Et}_{4} \mathrm{NCl}$ & $\mathrm{NR}^{* *}$ \\
$\mathbf{3}$ & $\mathrm{CHCl}_{3}$ & $24(3 \mathrm{~F})$ & $\mathrm{Et}_{4} \mathrm{NCl}$ & 11 \\
$\mathbf{4}$ & $\mathrm{DCM}$ & $24(3 \mathrm{~F})$ & $\mathrm{Et}_{4} \mathrm{NCl}$ & $\mathrm{NR}$ \\
$\mathbf{5}$ & $\mathrm{MeOH}$ & $24(3 \mathrm{~F})$ & $\mathrm{Et}{ }_{4} \mathrm{NCl}$ & 4 \\
$\mathbf{6}$ & $\mathrm{MeOH}$ & $24(3 \mathrm{~F})$ & $\mathrm{KOtBu}-\mathrm{Base}$ & $\mathrm{NR}$ \\
$\mathbf{7}$ & $\mathrm{TFE}$ & $24(3 \mathrm{~F})$ & Et & 6 \\
$\mathbf{8}$ & $\mathrm{HFIP}$ & $24(3 \mathrm{~F})$ & Et $\mathrm{NCl}$ & 25 \\
$\mathbf{9}$ & $\mathrm{HFIP}$ & $24(3 \mathrm{~F})$ & Without supporting & 63 \\
& & electrolyte & \\
\hline
\end{tabular}

Table S6. The conversion determined using ${ }^{1} \mathrm{H}$ NMR spectroscopy

$\mathrm{NR}=$ No reaction $=$ Starting materials recovered

${ }^{* *}$ precipitated inside the reactor 


\subsubsection{Electrolyte screening}

\begin{tabular}{|c|c|c|c|c|}
\hline \multirow[b]{2}{*}{ Entry } & \multicolumn{3}{|c|}{$\begin{array}{c}\mathrm{Pt}(+) / \mathrm{Pt}(-) \\
\mathrm{HFIP}, 0.1 \mathrm{~mL} \mathrm{~min}^{-1} \\
\text { reactor volume } 0.3 \mathrm{~mL}\end{array}$} & $\mathrm{O}_{1}=\mathrm{NCN}$ \\
\hline & \multicolumn{2}{|c|}{ Electrolyte } & Current (mA) & Conversion (\%) \\
\hline 1 & \multicolumn{2}{|l|}{$\mathrm{Et}_{4} \mathrm{NI}$} & $24(1.5 \mathrm{~F})$ & $\mathrm{NR}$ \\
\hline 2 & \multicolumn{2}{|c|}{$\mathrm{Et}_{4} \mathrm{NBr}$} & $24(1.5 \mathrm{~F})$ & NR \\
\hline 3 & \multicolumn{2}{|c|}{$\mathrm{n}-\mathrm{Bu}{ }_{4} \mathrm{NBr}$} & $24(1.5 \mathrm{~F})$ & NR \\
\hline
\end{tabular}

Table S7. The conversion determined using ${ }^{1} \mathrm{H}$ NMR spectroscopy

$\mathrm{NR}=$ No reaction $=$ Starting materials recovered

\subsubsection{Flow rate screening}

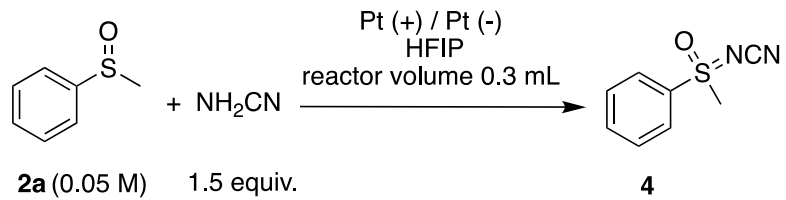

\begin{tabular}{cccc} 
Entry & Flow Rate $(\mathbf{m l} / \mathbf{m i n})$ & Current $(\mathbf{m A})$ & Conversion $(\%)$ \\
\hline $\mathbf{1}$ & 0.075 & $18(3 \mathrm{~F})$ & 33 \\
$\mathbf{2}$ & 0.075 & $24(4 \mathrm{~F})$ & 48 \\
$\mathbf{3}$ & 0.075 & $36(6 \mathrm{~F})$ & 62 \\
$\mathbf{4}$ & 0.1 & $24(3 \mathrm{~F})$ & 34 \\
$\mathbf{5}$ & 0.1 & $32(4 \mathrm{~F})$ & 41 \\
$\mathbf{6}$ & 0.1 & $48(6 \mathrm{~F})$ & 63 \\
$\mathbf{7}$ & 0.15 & $36(3 \mathrm{~F})$ & 33 \\
$\mathbf{8}$ & 0.15 & $48(4 \mathrm{~F})$ & 48 \\
$\mathbf{9}$ & 0.15 & $72(6 \mathrm{~F})$ & 68 \\
$\mathbf{1 0}$ & 0.2 & $48(3 \mathrm{~F})$ & 17 \\
$\mathbf{1 1}$ & 0.2 & $64(4 \mathrm{~F})$ & 42 \\
$\mathbf{1 2}$ & 0.2 & $96(6 \mathrm{~F})$ & 62 \\
\hline
\end{tabular}

Table S8. The conversion determined using ${ }^{1} \mathrm{H}$ NMR spectroscopy 


\subsubsection{Sulfoxide concentration screening}

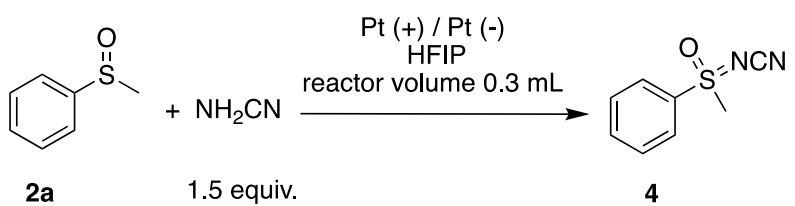

\begin{tabular}{ccccc} 
Entry & Concentration (M) & Current $(\mathbf{m A})$ & Voltage (V) & Conversion (\%) \\
\hline 1 & 0.025 & 36 & $11-13$ & 29 \\
2 & 0.05 & 72 & $13-15$ & 68 \\
3 & 0.1 & 144 & $33-35$ & $62^{* *}$
\end{tabular}

Table S8. The conversion determined using ${ }^{1} \mathrm{H}$ NMR spectroscopy

** The voltage was high and the current was unstable

\section{General procedures}

\subsection{General procedure for the electrochemical oxidation of sulfides to sulfoxides (GP1):}

The electrolysis was performed in an undivided cell using a Vapourtec Ion Electrochemical Flow Reactor (reactor volume $=0.6 \mathrm{~mL}$, spacer $0.5 \mathrm{~mm}$ ) using a graphite $(\mathrm{Gr}$ ) electrode as the anode and a stainless steel ( $F e$ ) electrode as the cathode (active surface area: $A=12 \mathrm{~cm}^{2}$ ). A solution of sulfide $(0.1 \mathrm{M})$ in mixture of $\mathrm{CH}_{3} \mathrm{CN}$ and $\mathrm{H}_{2} \mathrm{O}(9: 1)$ placed in $20 \mathrm{~mL}$ vial screw cap with hole with PTFE/silicone septum. The reaction mixture was injected to $18 \mathrm{~mL}$ sample loop. After that, the reactor temperature was set at room temperature with the flow rate $0.2 \mathrm{~mL} / \mathrm{min}$ and the current was set at $64 \mathrm{~mA}$ turn on automatically. Then, solutions were pumped into the electrochemical reactor. After reaching a steady state, the solution $(15 \mathrm{~mL})$ was collected automatically into a glass vial. The solvent was removed under vacuum. The crude product was purified by column chromatography (EtOAc/cyclohexane).

\subsection{General procedure for the electrochemical oxidation of sulfides to sulfones (GP2):}

The electrolysis was performed in an undivided cell using a Vapourtec lon Electrochemical Flow Reactor (reactor volume $=0.6 \mathrm{~mL}$, spacer $0.5 \mathrm{~mm}$ ) using a graphite $(\mathrm{Pt}$ ) electrode as the anode and a stainless steel ( $\mathrm{Fe}$ ) electrode as the cathode (active surface area: $A=12 \mathrm{~cm}^{2}$ ). A solution of sulfide $(0.1 \mathrm{M})$ in mixture of $\mathrm{CH}_{3} \mathrm{CN}$ and $\mathrm{H}_{2} \mathrm{O}(9: 1)$ placed in $20 \mathrm{~mL}$ vial screw cap with hole 
with PTFE/silicone septum. The reaction mixture was injected to the $18 \mathrm{~mL}$ sample loop. After that, the reactor temperature was set at room temperature with the flow rate $0.2 \mathrm{~mL} / \mathrm{min}$ and the current was set at $160 \mathrm{~mA}$ turn on automatically. Then, solutions were pumped into the electrochemical rector. After reaching a steady state, the solution $(15 \mathrm{~mL})$ was collected automatically into a glass vial. The solvent was removed under vacuum. The crude product was purified by column chromatography (EtOAc/cyclohexane).

\subsection{General procedure for the electrochemical imination of sulfoxides (GP3):}

The electrolysis was performed in an undivided cell using a Vapourtec lon Electrochemical Flow Reactor (reactor volume $=0.3 \mathrm{~mL}$, spacer $0.3 \mathrm{~mm}$ ) using a platinum $(\mathrm{Pt}$ ) electrode as anode and cathode (active surface area: $\left.A=12 \mathrm{~cm}^{2}\right)$. A solution of sulfoxide $(0.05 \mathrm{M})$ and cyanamide (1.5 equiv.) in HFIP (12 mL) was placed in a $20 \mathrm{~mL}$ vial screw cap with hole with PTFE/silicone septum. The reaction mixture was injected to the $12 \mathrm{~mL}$ sample loop. After that, the reactor temperature was set at room temperature with the flow rate $0.15 \mathrm{~mL} / \mathrm{min}$ and the current was set at $72 \mathrm{~mA}$ turn on automatically. Then, solutions were pumped into the electrochemical rector. After reaching a steady state, the solution $(10 \mathrm{~mL})$ was collected automatically into a glass vial. The solvent was removed under vacuum. The crude product was purified by column chromatography (EtOAc/cyclohexane).

\subsection{General procedure for the reduction of $\mathbf{N}$-cyanosulfoximine (GP4):}

To a mixture solution of $\mathrm{N}$-cyanosulfoximine $(0.5 \mathrm{mmol})$ in $\mathrm{CH}_{2} \mathrm{Cl}_{2}(10 \mathrm{~mL})$ at $0{ }^{\circ} \mathrm{C}$, TFAA $(1.5 \mathrm{mmol})$ was added. Then, the reaction mixture was allowed to stirrer at room temperature until the starting material was consumed (monitored by TLC). The mixture was quenched with water $(10 \mathrm{~mL})$. The aqueous layer was extracted with $\mathrm{CH} 2 \mathrm{Cl} 2(3 \times 10 \mathrm{~mL})$. The combined organic layers were dried over anhydrous $\mathrm{MgSO} 4$, filtered and evaporated. The residue was purified by column chromatography (EtOAc/ Cyclohexane). 
6. NMR spectra

${ }^{1} \mathrm{H}$ NMR $\left(500 \mathrm{MHz}, \mathrm{CDCl}_{3}, 298 \mathrm{~K}\right)$

(methylsulfinyl)benzene (2a)

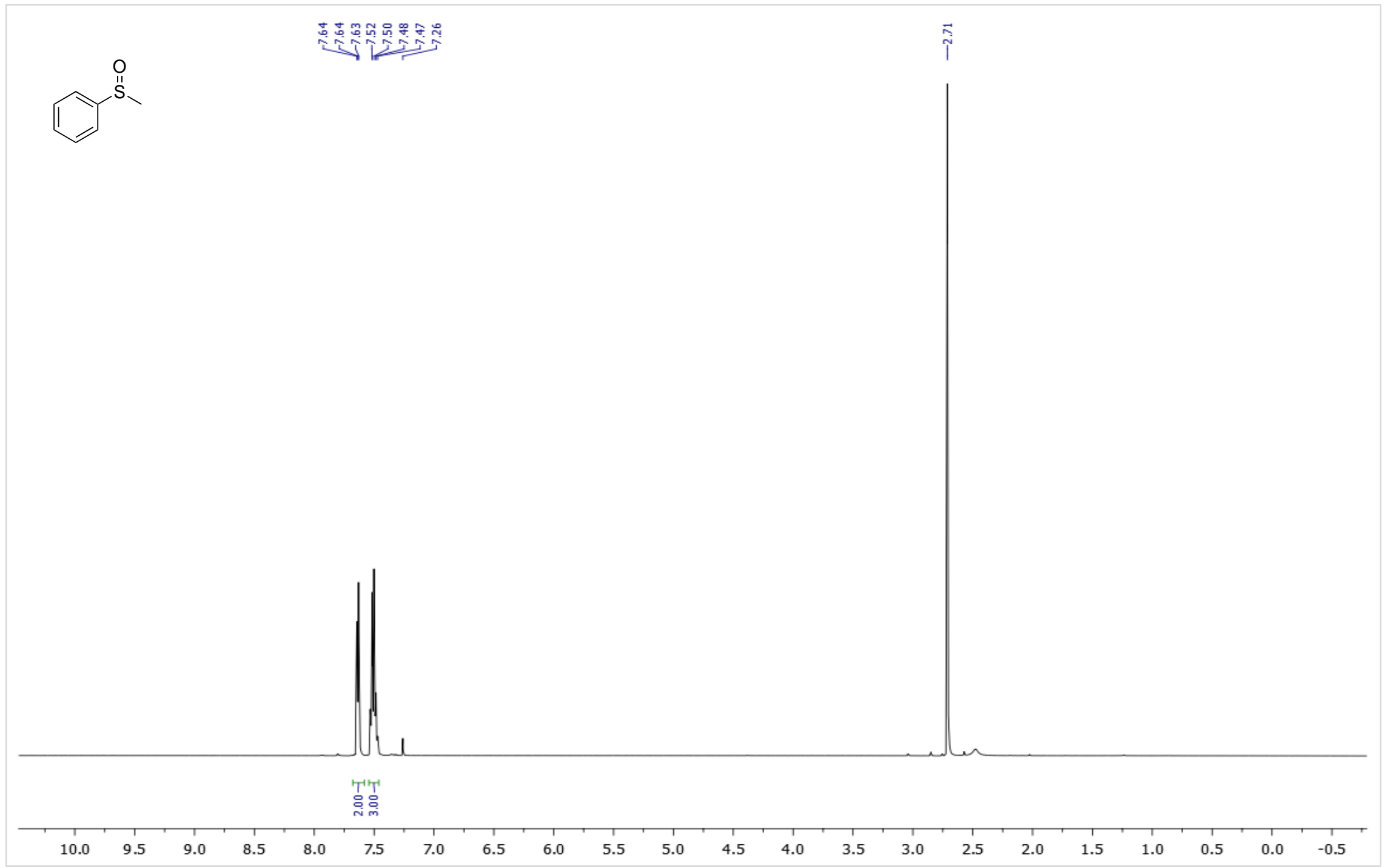


${ }^{13} \mathrm{C}\left\{{ }^{1} \mathrm{H}\right\}$ NMR $\left(126 \mathrm{MHz}, \mathrm{CDCl}_{3}, 298 \mathrm{~K}\right)$

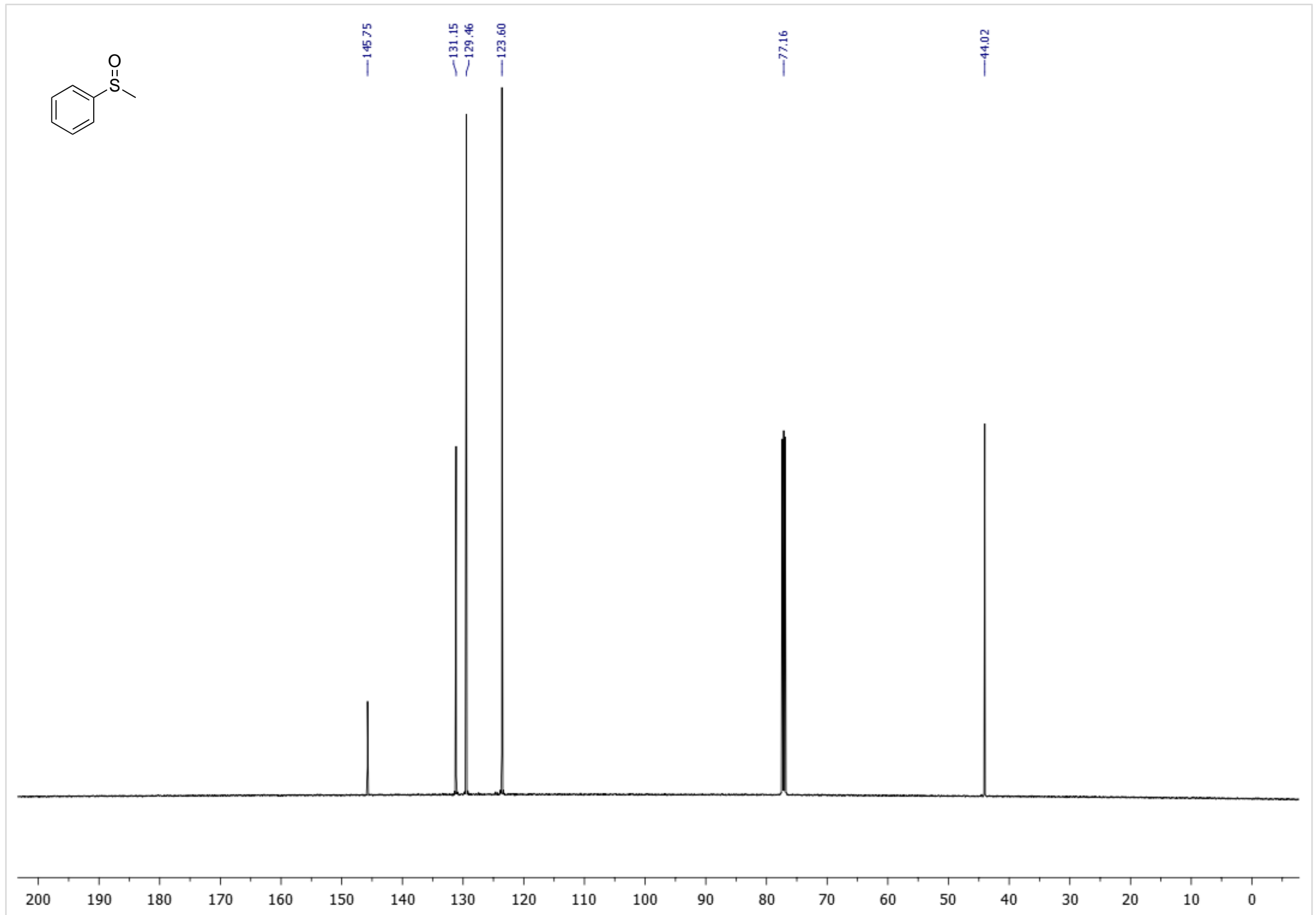


1-methyl-4-(methylsulfinyl)benzene (2b)

${ }^{1} \mathbf{H}$ NMR $\left(500 \mathrm{MHz}, \mathrm{CDCl}_{3}, 298 \mathrm{~K}\right)$

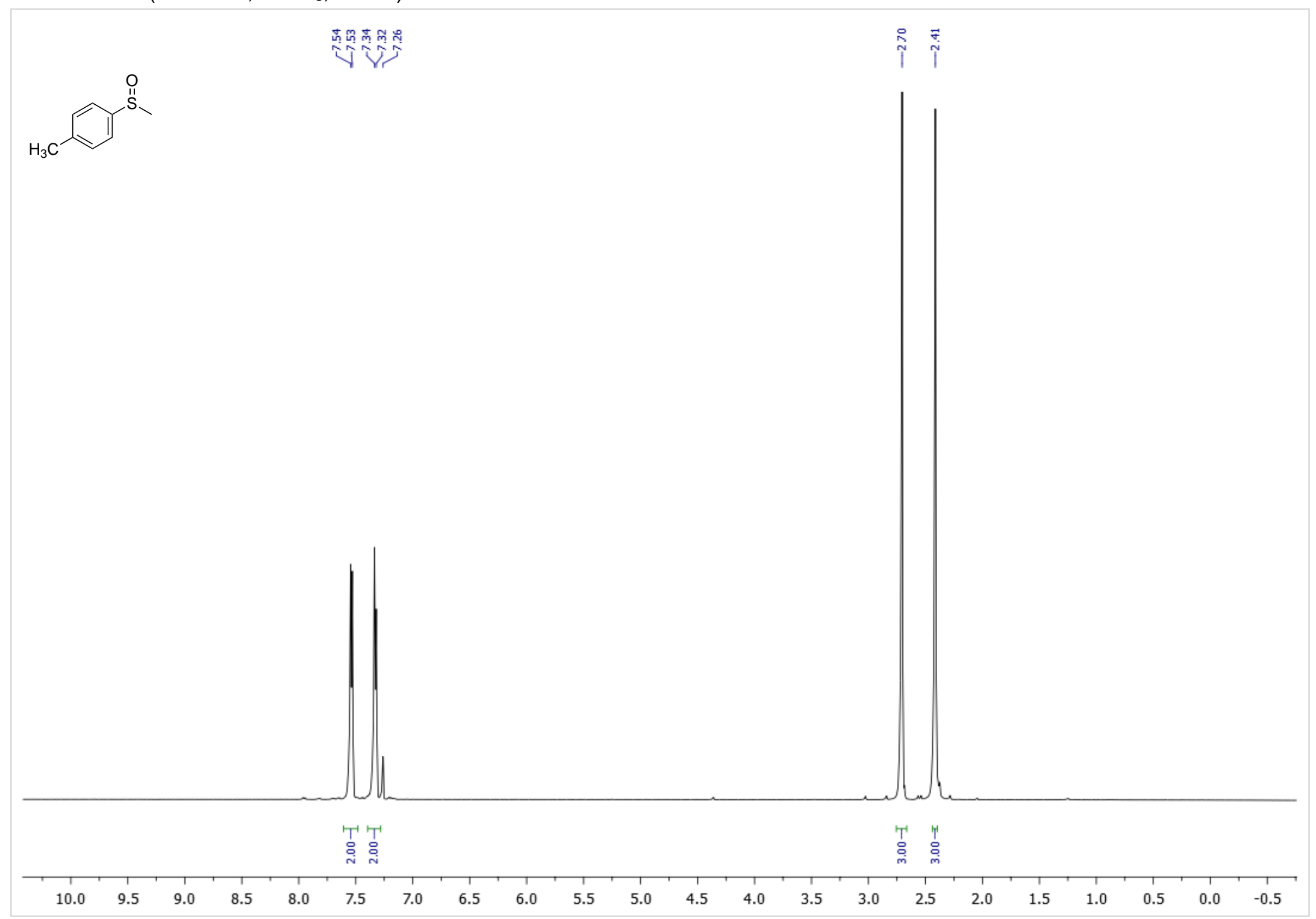


${ }^{13} \mathrm{C}\left\{{ }^{1} \mathrm{H}\right\}$ NMR $\left(126 \mathrm{MHz}, \mathrm{CDCl}_{3}, 298 \mathrm{~K}\right)$

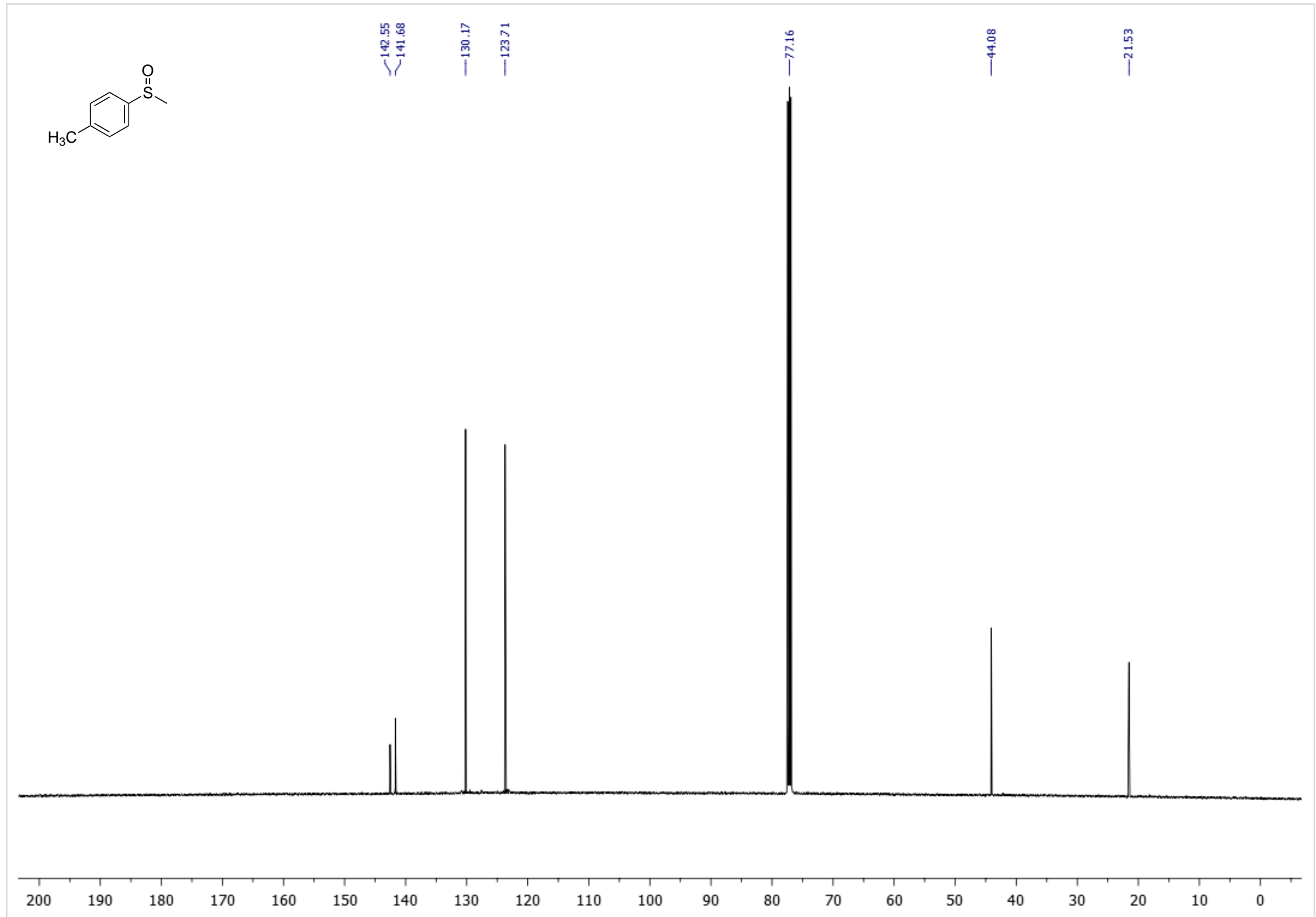


1-bromo-4-(methylsulfinyl)benzene (2c)

${ }^{1} \mathbf{H}$ NMR $\left(500 \mathrm{MHz}, \mathrm{CDCl}_{3}, 298 \mathrm{~K}\right)$

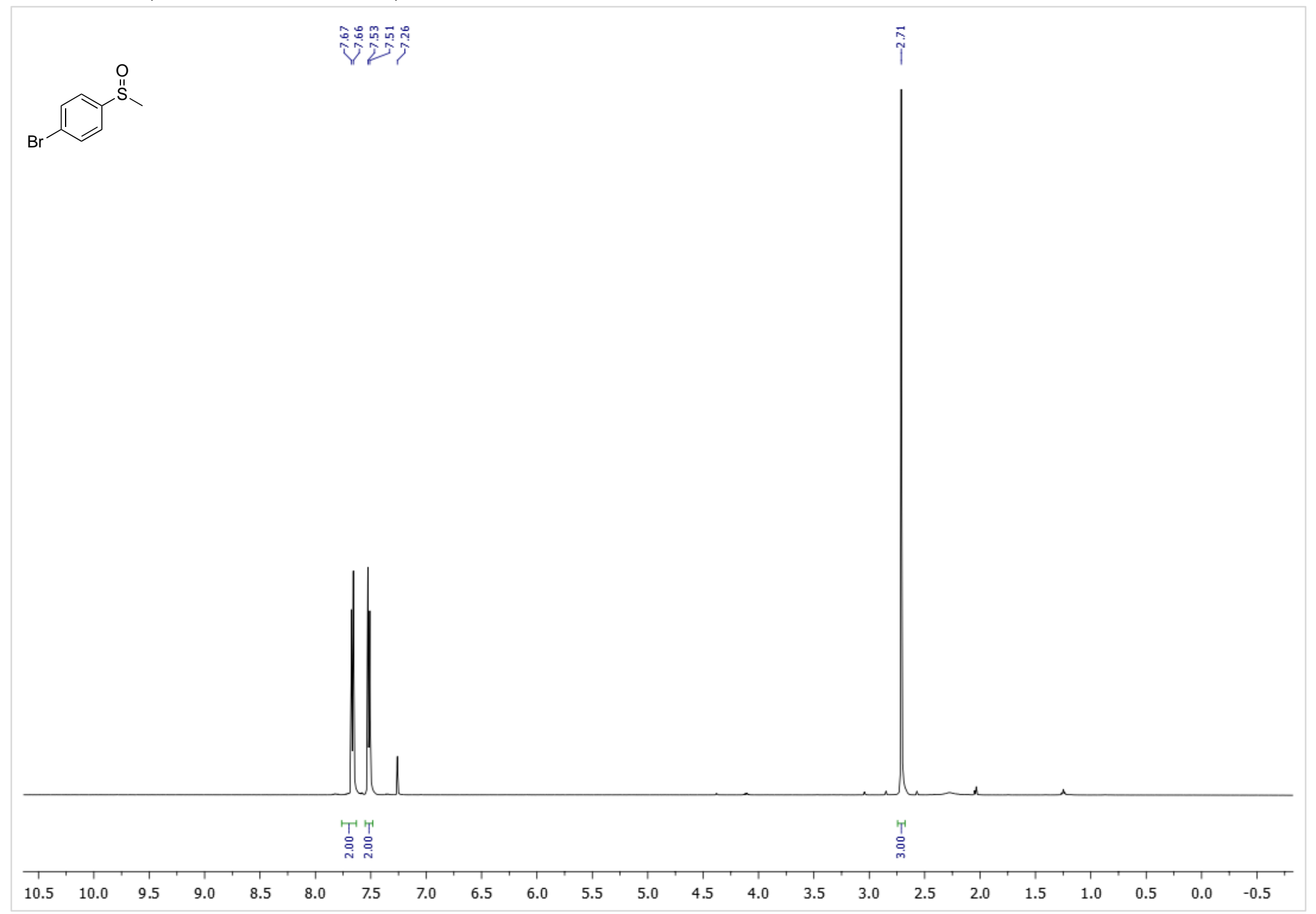


${ }^{13} \mathbf{C}\left\{{ }^{1} \mathrm{H}\right\}$ NMR $\left(126 \mathrm{MHz}, \mathrm{CDCl}_{3}, 298 \mathrm{~K}\right)$

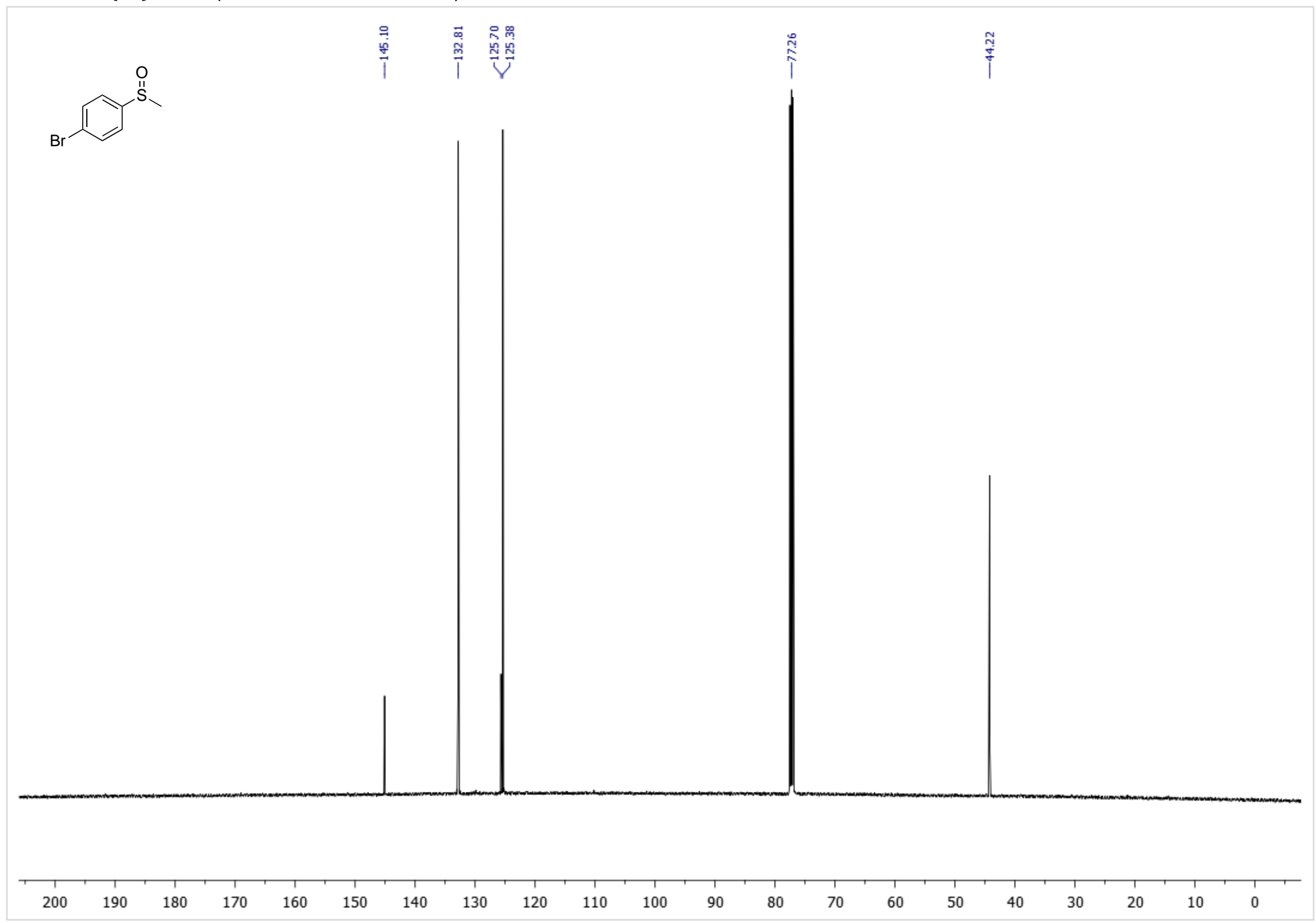

S16 
1-chloro-4-(methylsulfinyl)benzene (2d)

${ }^{1} \mathbf{H}$ NMR $\left(500 \mathrm{MHz}, \mathrm{CDCl}_{3}, 298 \mathrm{~K}\right)$

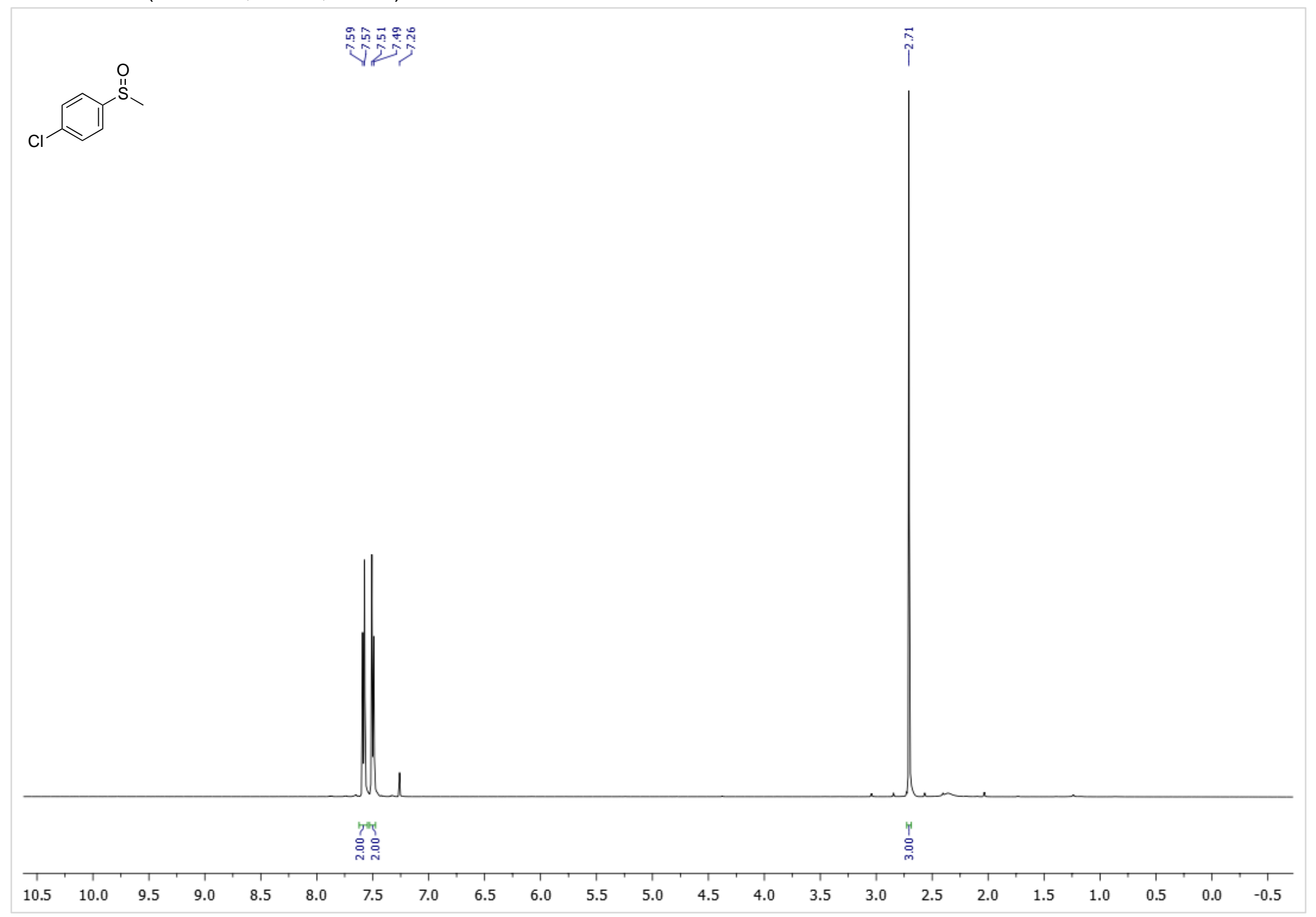


${ }^{13} \mathbf{C}\left\{{ }^{1} \mathrm{H}\right\}$ NMR $\left(126 \mathrm{MHz}, \mathrm{CDCl}_{3}, 298 \mathrm{~K}\right)$

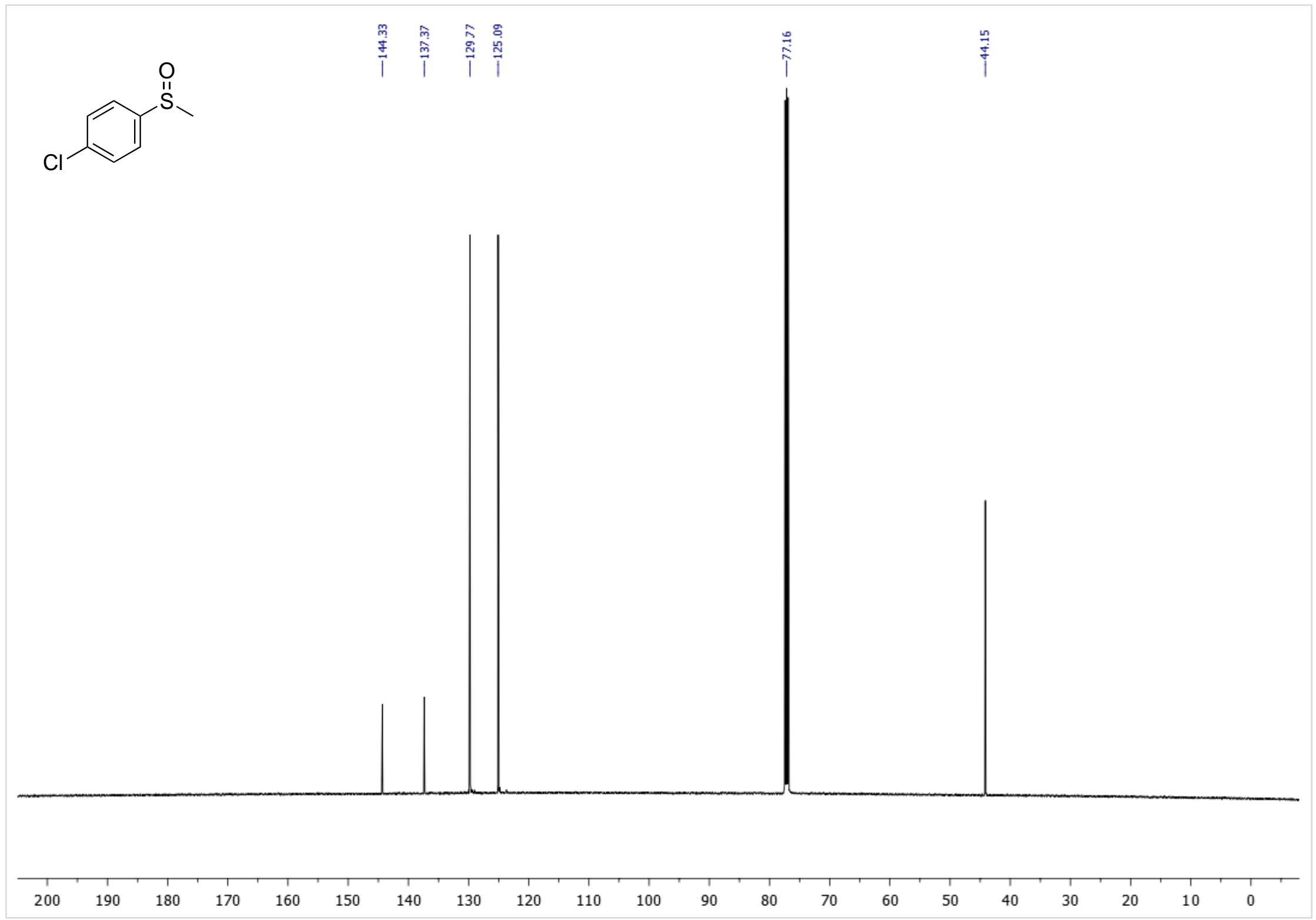

S18 
1-fluoro-4-(methylsulfinyl)benzene (2e)

${ }^{1} \mathbf{H}$ NMR $\left(500 \mathrm{MHz}, \mathrm{CDCl}_{3}, 298 \mathrm{~K}\right)$

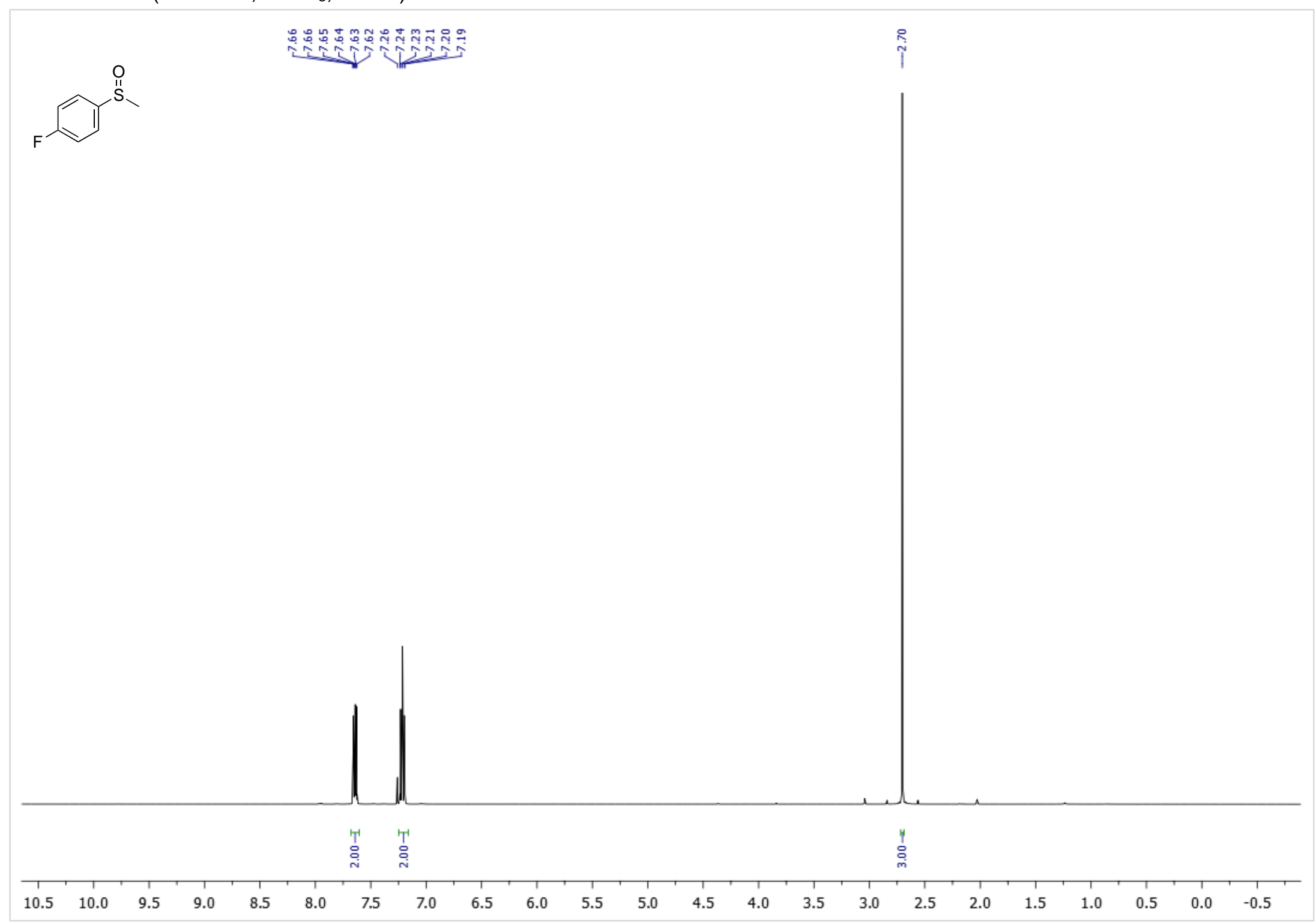


${ }^{13} \mathrm{C}\left\{{ }^{1} \mathrm{H}\right\}$ NMR $\left(126 \mathrm{MHz}, \mathrm{CDCl}_{3}, 298 \mathrm{~K}\right)$

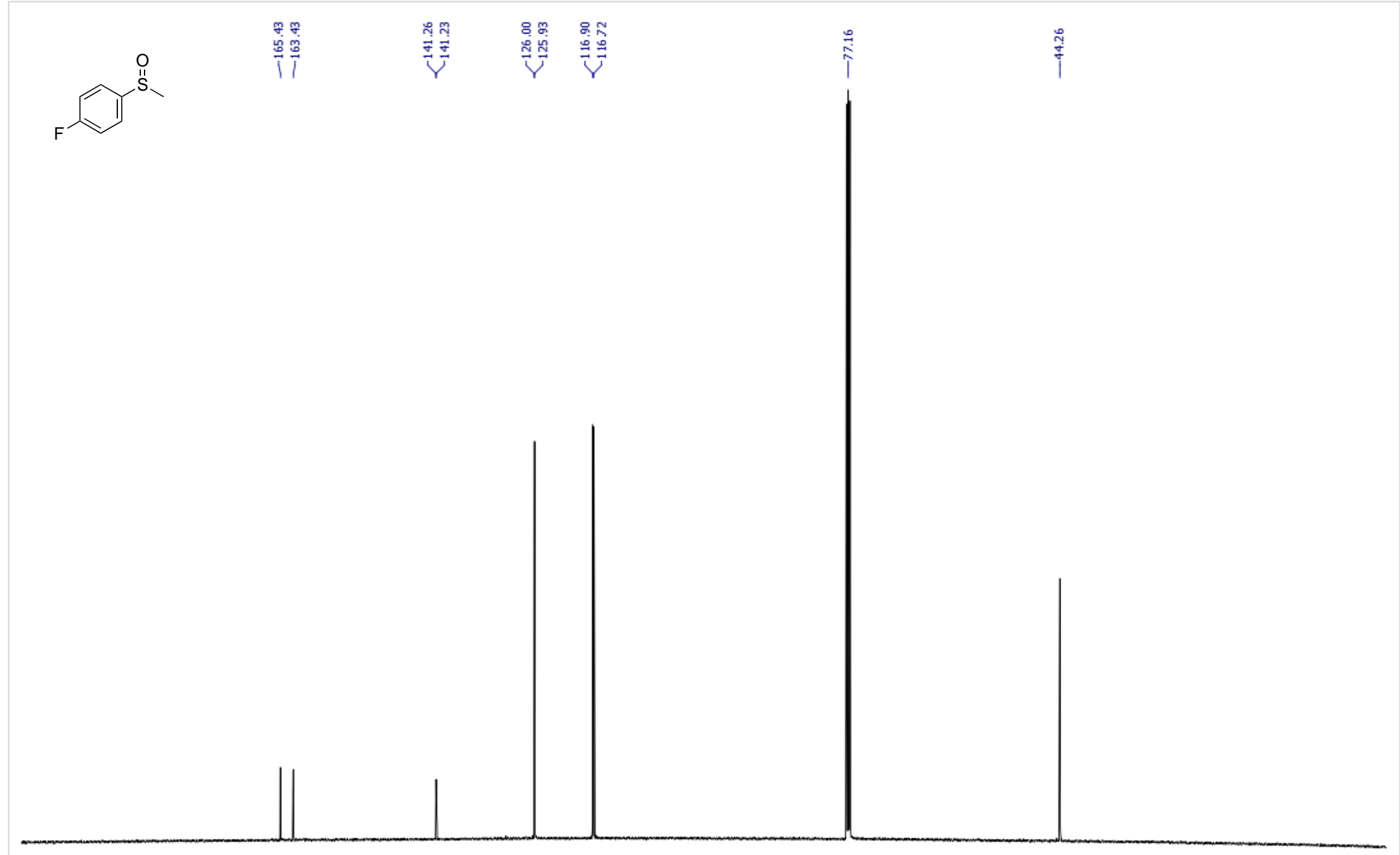

$$
200
$$


${ }^{19} \mathrm{~F}\left\{{ }^{1} \mathrm{H}\right\} \mathbf{N M R}\left(471 \mathrm{MHz}, \mathrm{CDCl}_{3}, 298 \mathrm{~K}\right)$

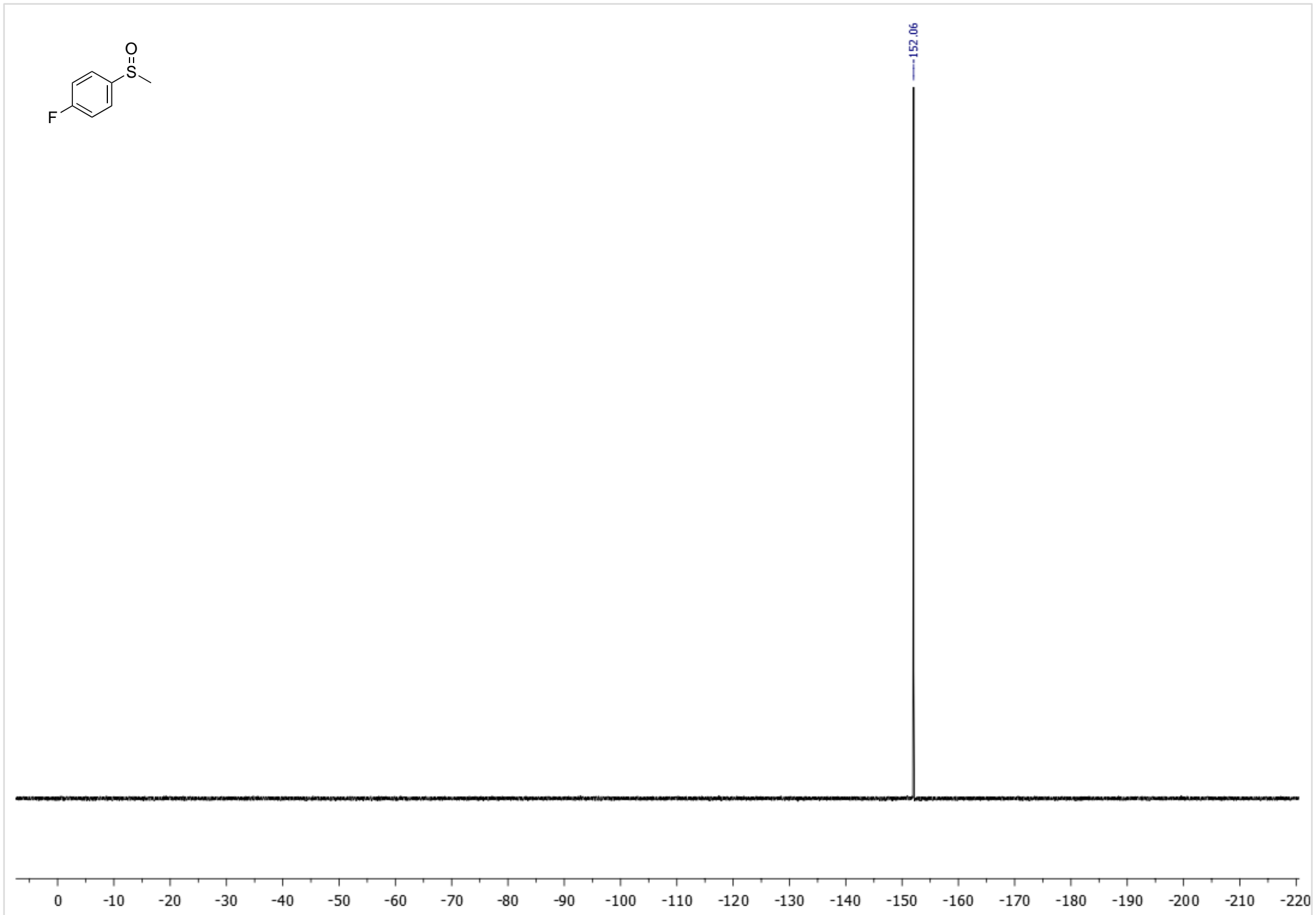


4-(methylsulfinyl)benzonitrile (2f)

${ }^{1} \mathrm{H}$ NMR $\left(500 \mathrm{MHz}, \mathrm{CDCl}_{3}, 298 \mathrm{~K}\right)$

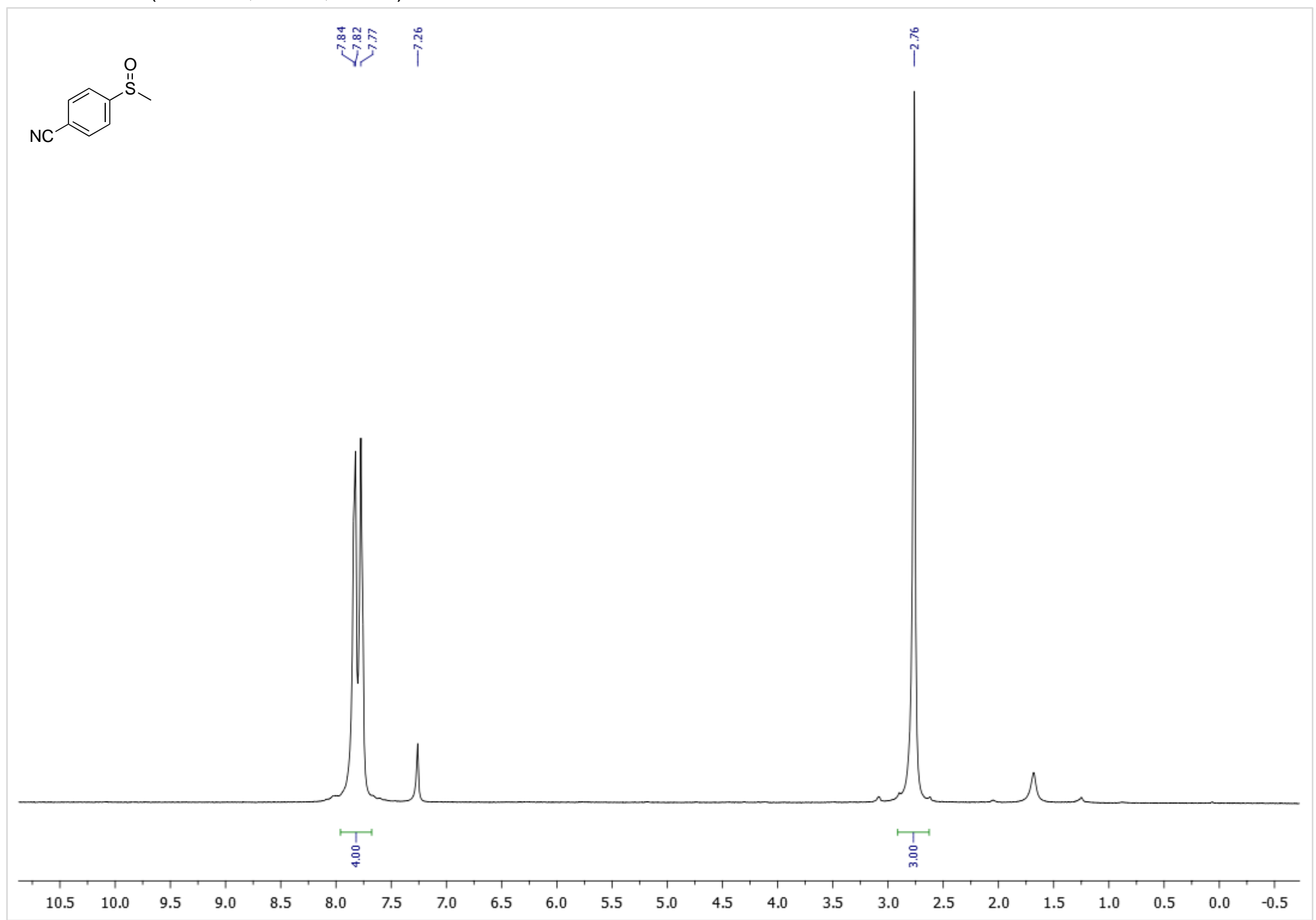


${ }^{13} \mathrm{C}\left\{{ }^{1} \mathrm{H}\right\}$ NMR $\left(126 \mathrm{MHz}, \mathrm{CDCl}_{3}, 298 \mathrm{~K}\right)$
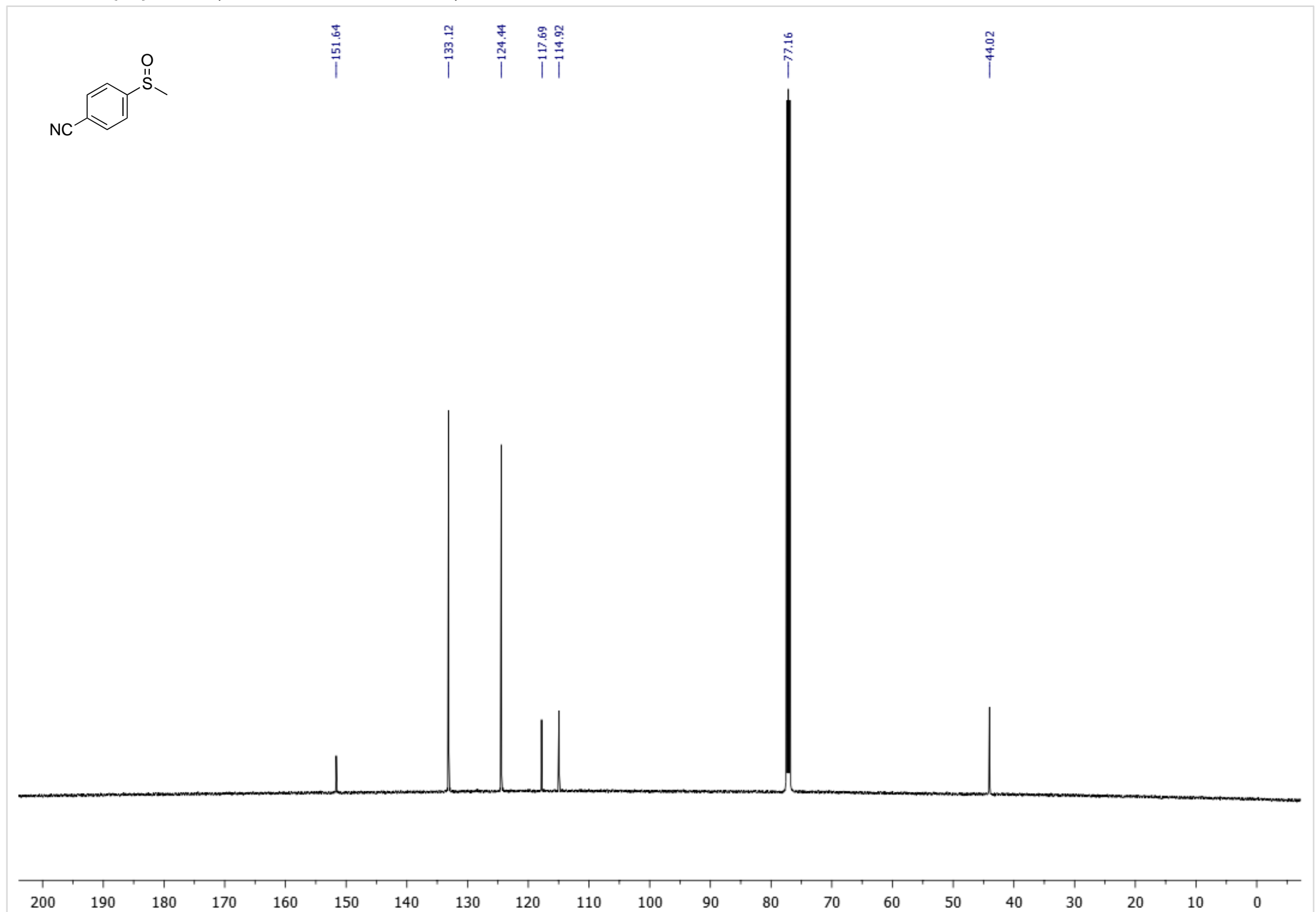
1-methoxy-4-(methylsulfinyl)benzene (2g)

${ }^{1} \mathbf{H}$ NMR $\left(400 \mathrm{MHz}, \mathrm{CDCl}_{3}, 298 \mathrm{~K}\right)$

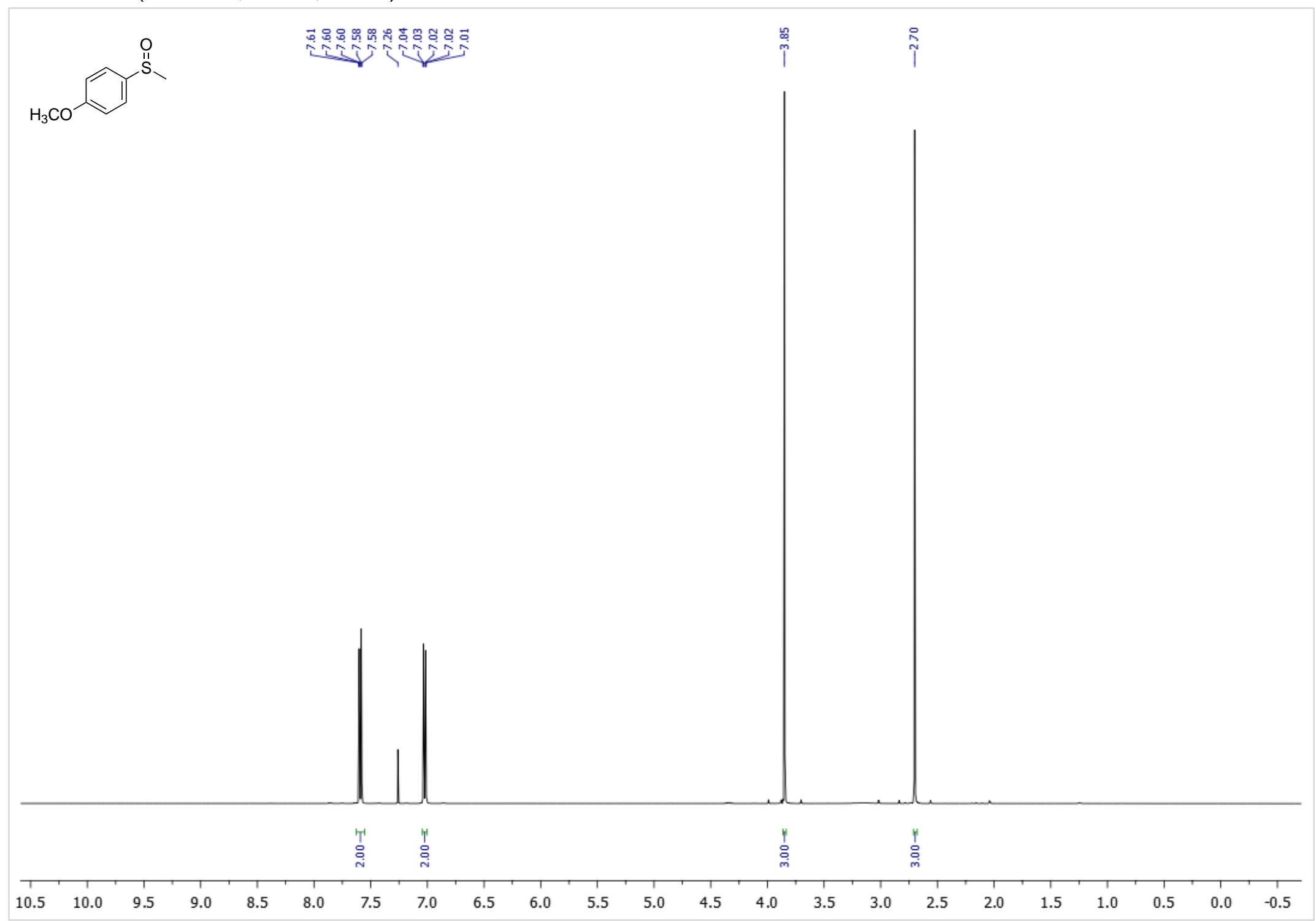


${ }^{13} \mathbf{C}\left\{{ }^{1} \mathrm{H}\right\}$ NMR $\left(101 \mathrm{MHz}, \mathrm{CDCl}_{3}, 298 \mathrm{~K}\right)$

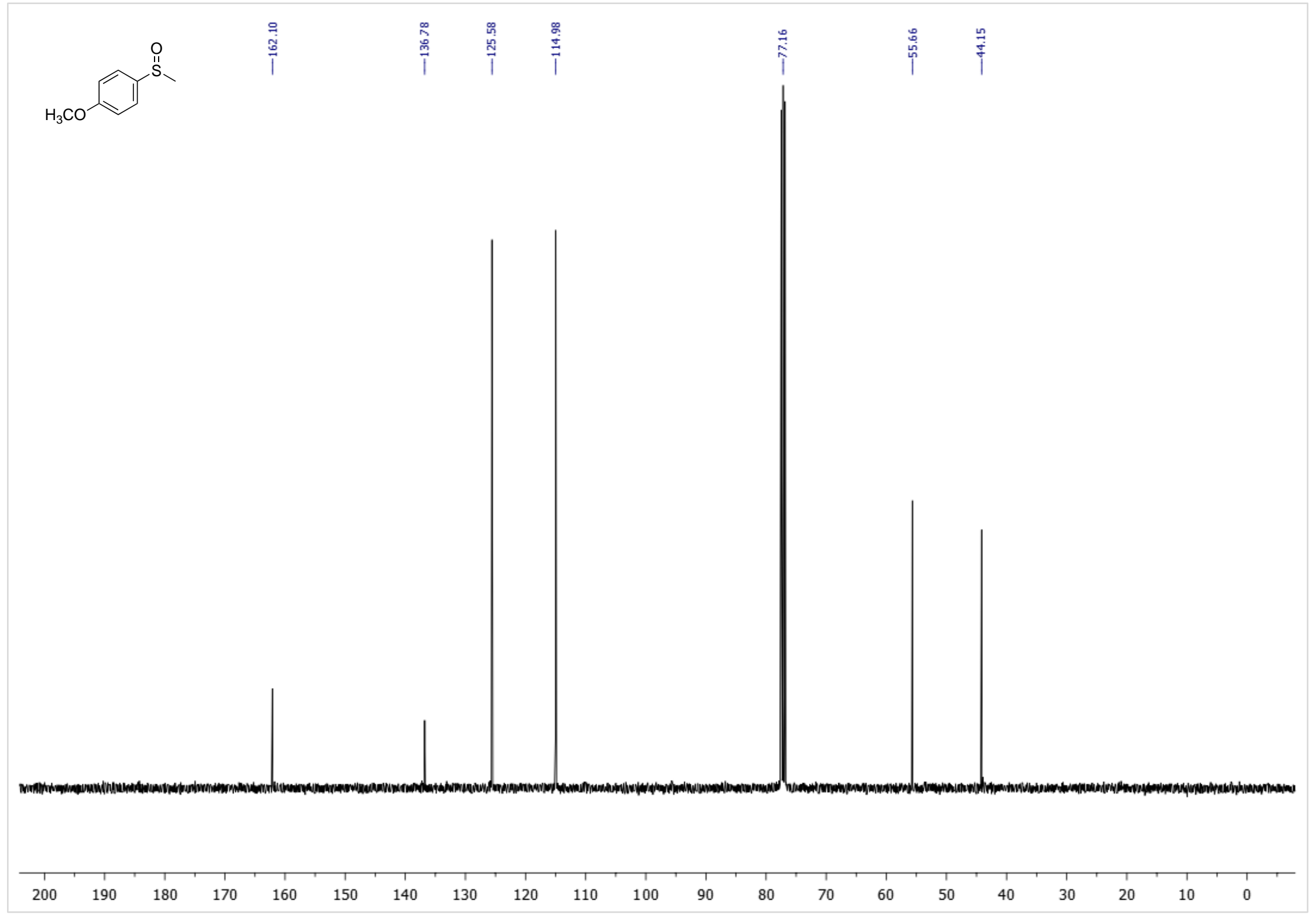

S25 
4-(methylsulfinyl)benzaldehyde (2h)

${ }^{\mathbf{1}} \mathbf{H}$ NMR $\left(500 \mathrm{MHz}, \mathrm{CDCl}_{3}, 298 \mathrm{~K}\right)$

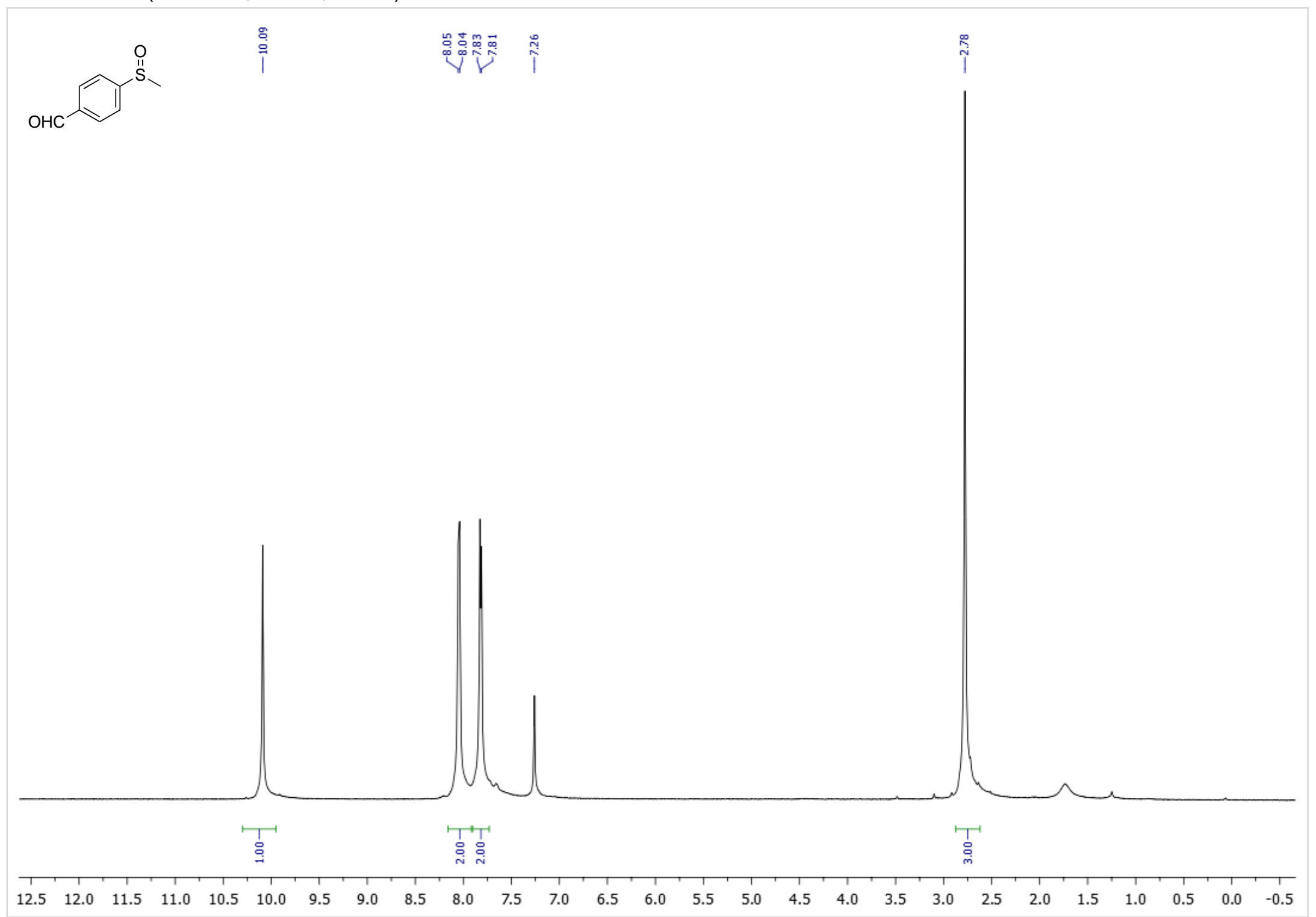


${ }^{13} \mathrm{C}\left\{{ }^{1} \mathrm{H}\right\}$ NMR $\left(126 \mathrm{MHz}, \mathrm{CDCl}_{3}, 298 \mathrm{~K}\right)$

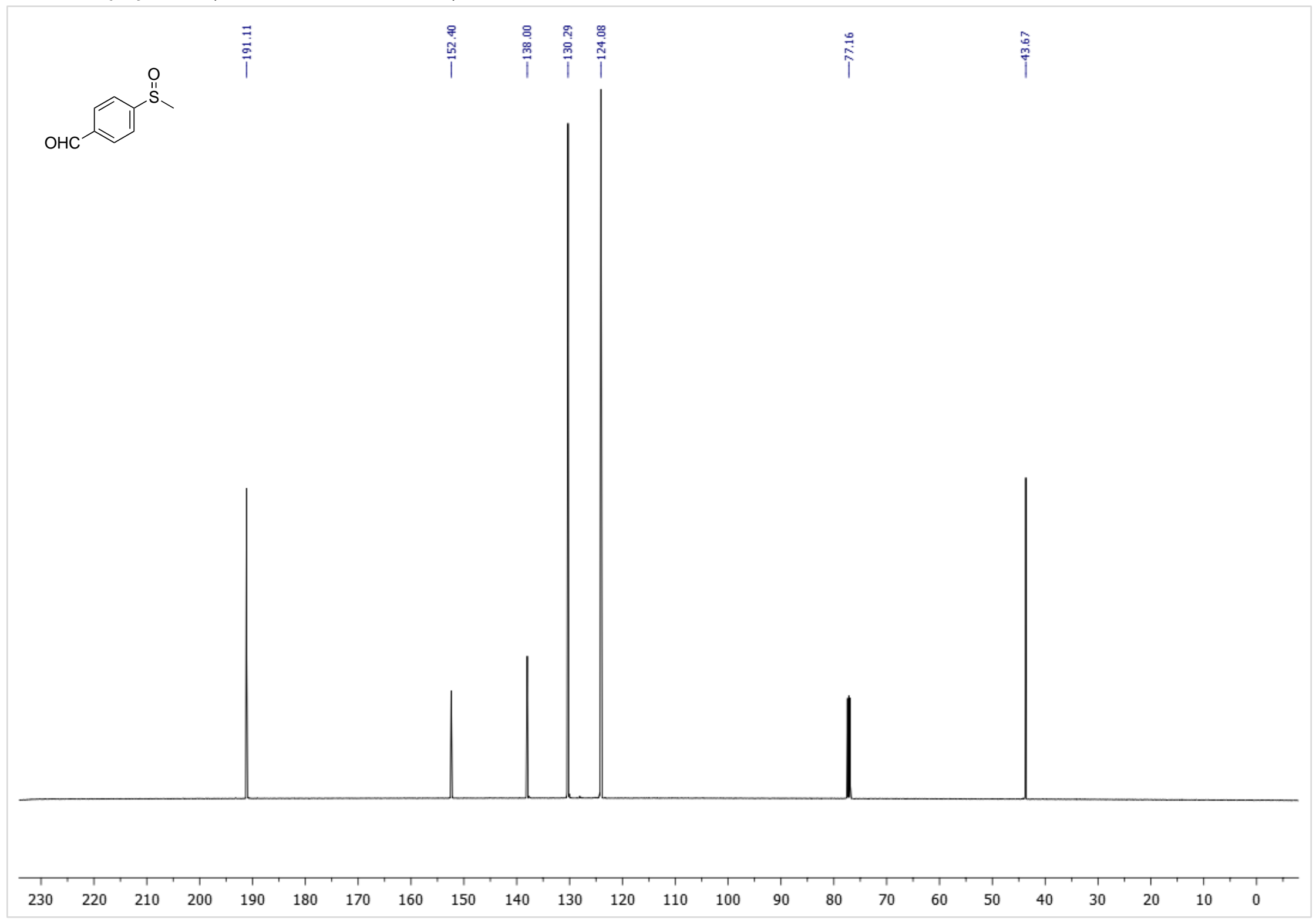

S27 
1-(methylsulfinyl)-4-nitrobenzene (2i)

${ }^{1} \mathrm{H}$ NMR $\left(500 \mathrm{MHz}, \mathrm{CDCl}_{3}, 298 \mathrm{~K}\right)$

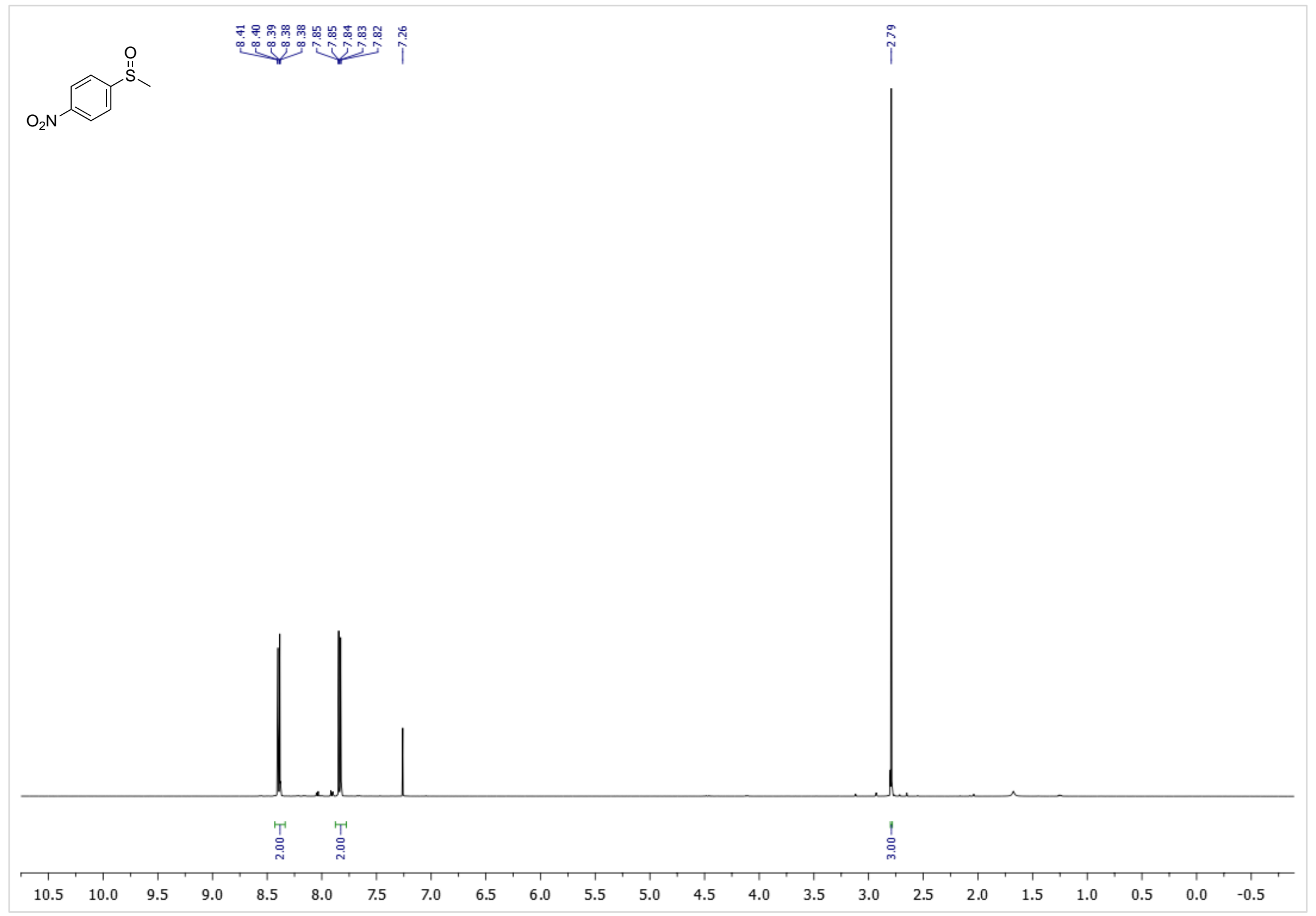


${ }^{13} \mathrm{C}\left\{{ }^{1} \mathrm{H}\right\}$ NMR $\left(126 \mathrm{MHz}, \mathrm{CDCl}_{3}, 298 \mathrm{~K}\right)$

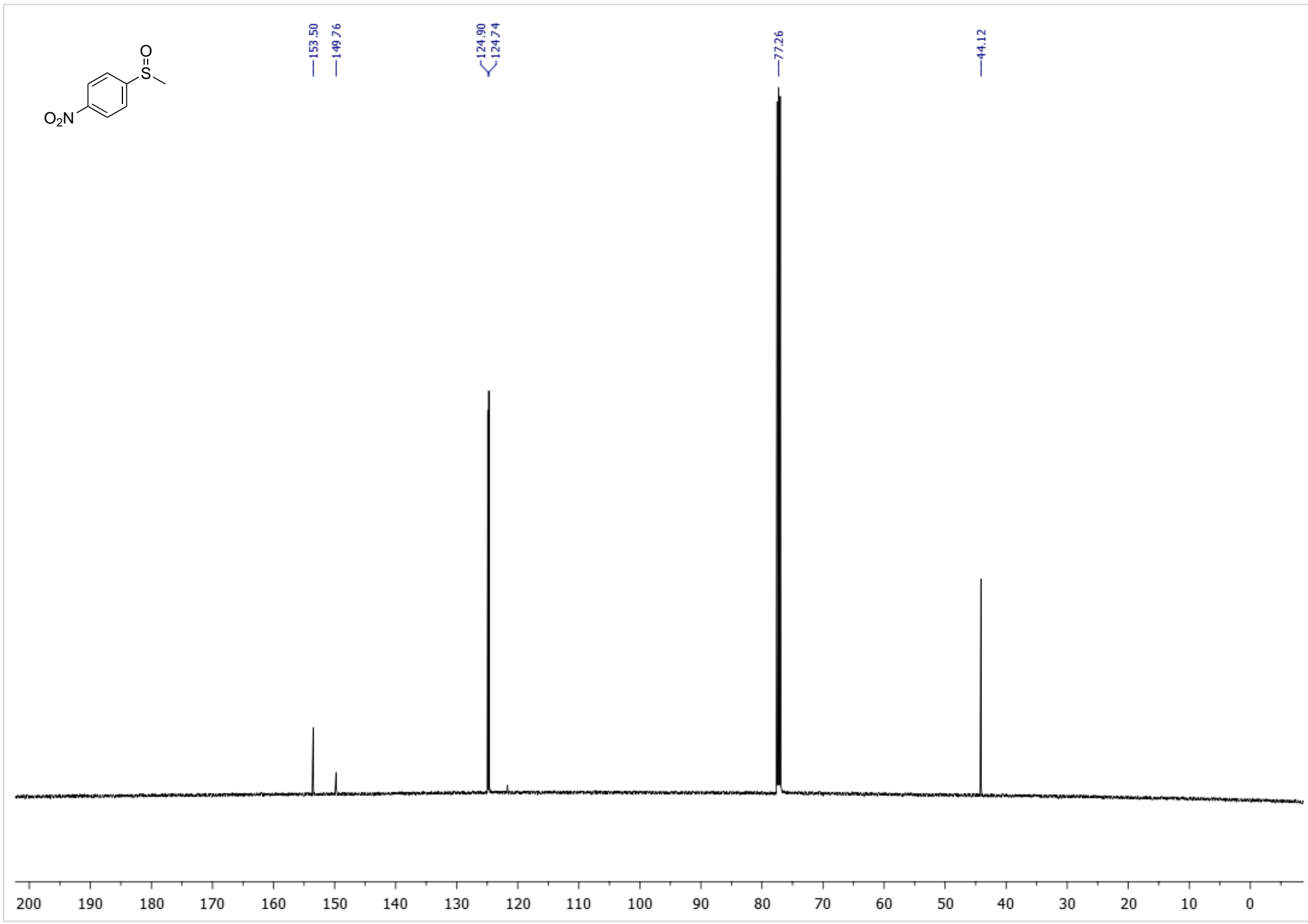


1-methyl-3-(methylsulfinyl)benzene (2j)

${ }^{1} \mathbf{H}$ NMR $\left(500 \mathrm{MHz}, \mathrm{CDCl}_{3}, 298 \mathrm{~K}\right)$

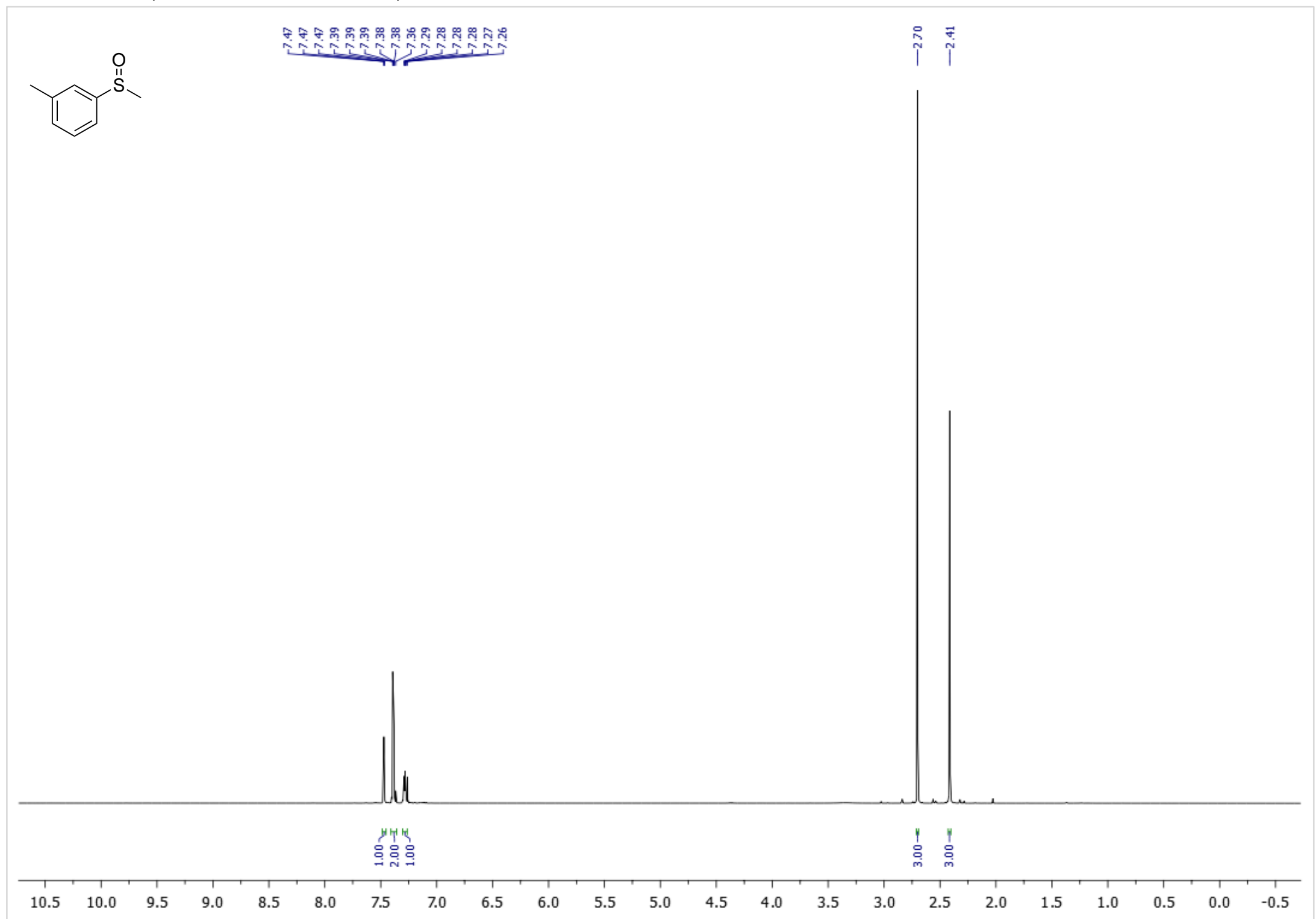


${ }^{13} \mathrm{C}\left\{{ }^{1} \mathrm{H}\right\}$ NMR $\left(126 \mathrm{MHz}, \mathrm{CDCl}_{3}, 298 \mathrm{~K}\right)$

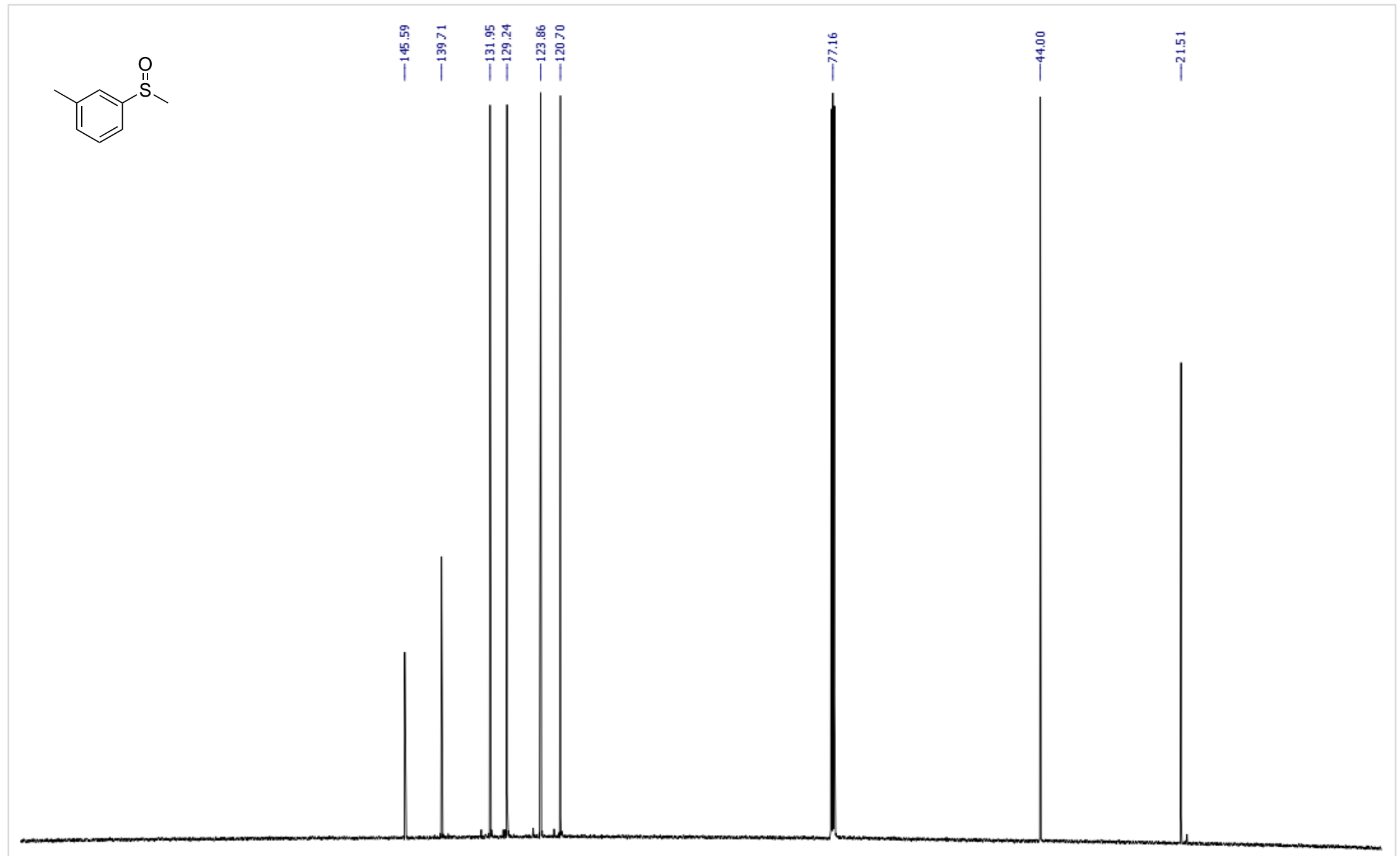


1-bromo-3-(methylsulfinyl)benzene (2k)

${ }^{1} \mathbf{H}$ NMR (500 MHz, $\left.\mathrm{CDCl}_{3}, 298 \mathrm{~K}\right)$

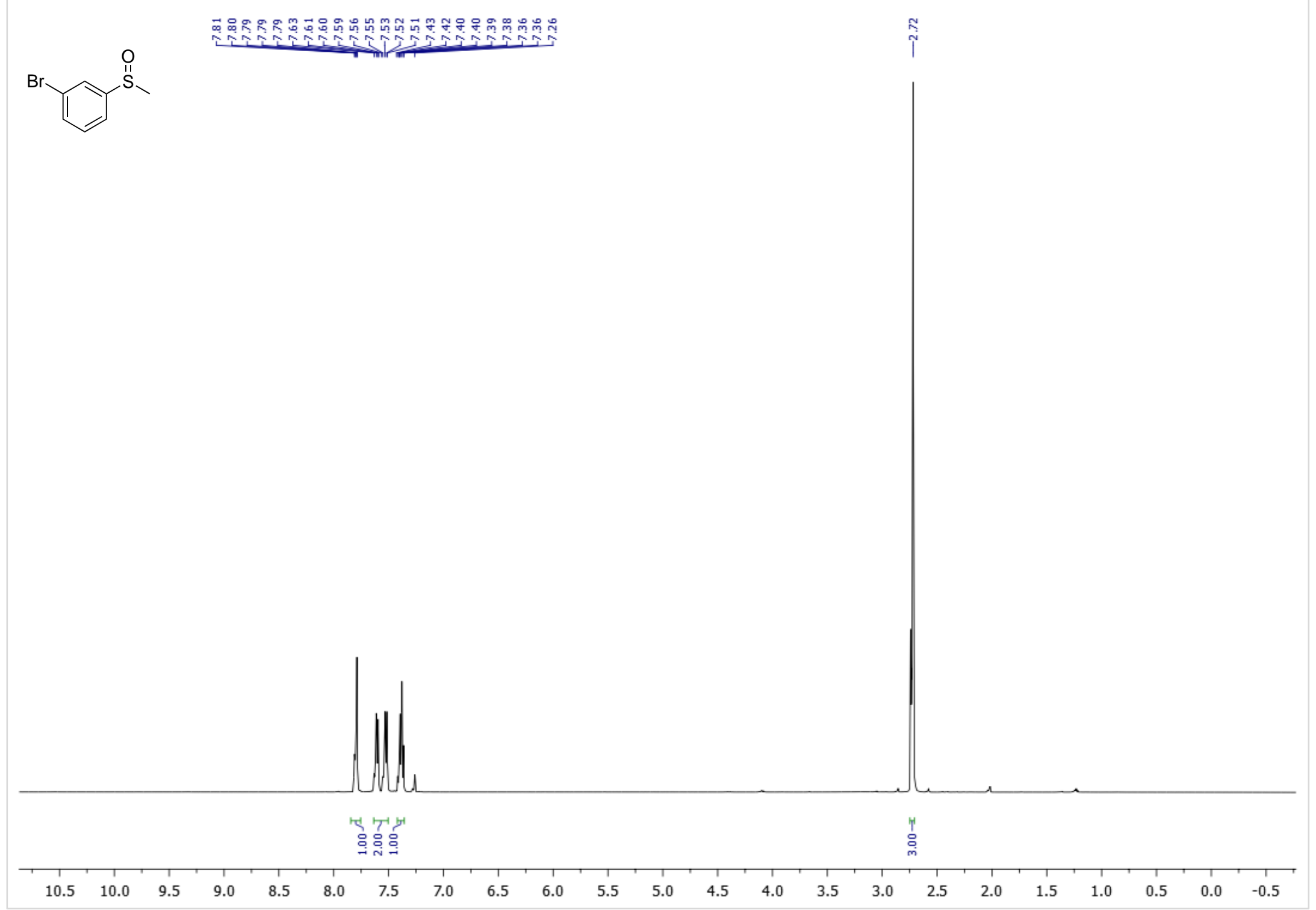


${ }^{13} \mathrm{C}\left\{{ }^{1} \mathrm{H}\right\}$ NMR $\left(126 \mathrm{MHz}, \mathrm{CDCl}_{3}, 298 \mathrm{~K}\right)$

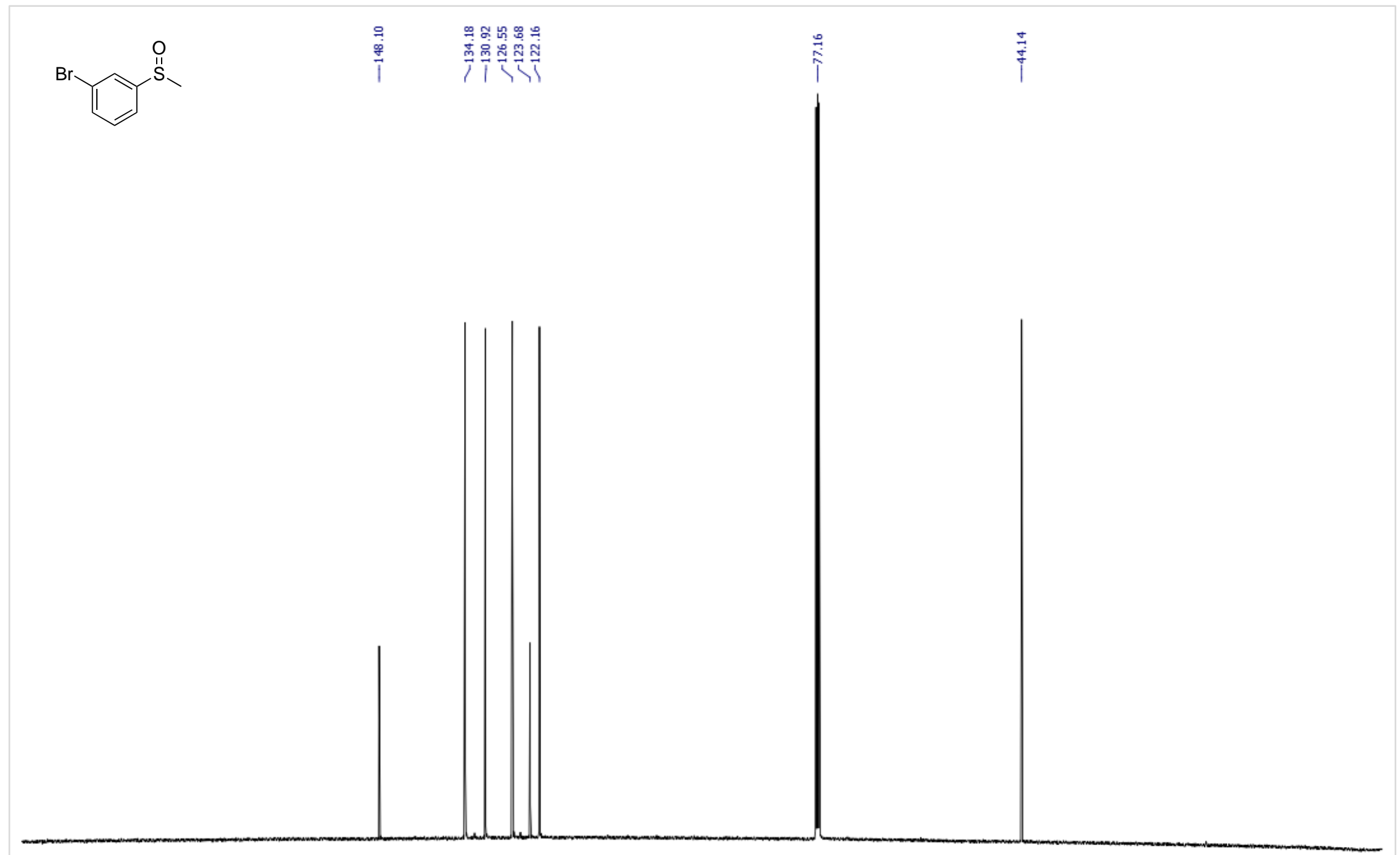


1-chloro-3-(methylsulfinyl)benzene (2I)

${ }^{1} \mathbf{H}$ NMR $\left(500 \mathrm{MHz}, \mathrm{CDCl}_{3}, 298 \mathrm{~K}\right)$

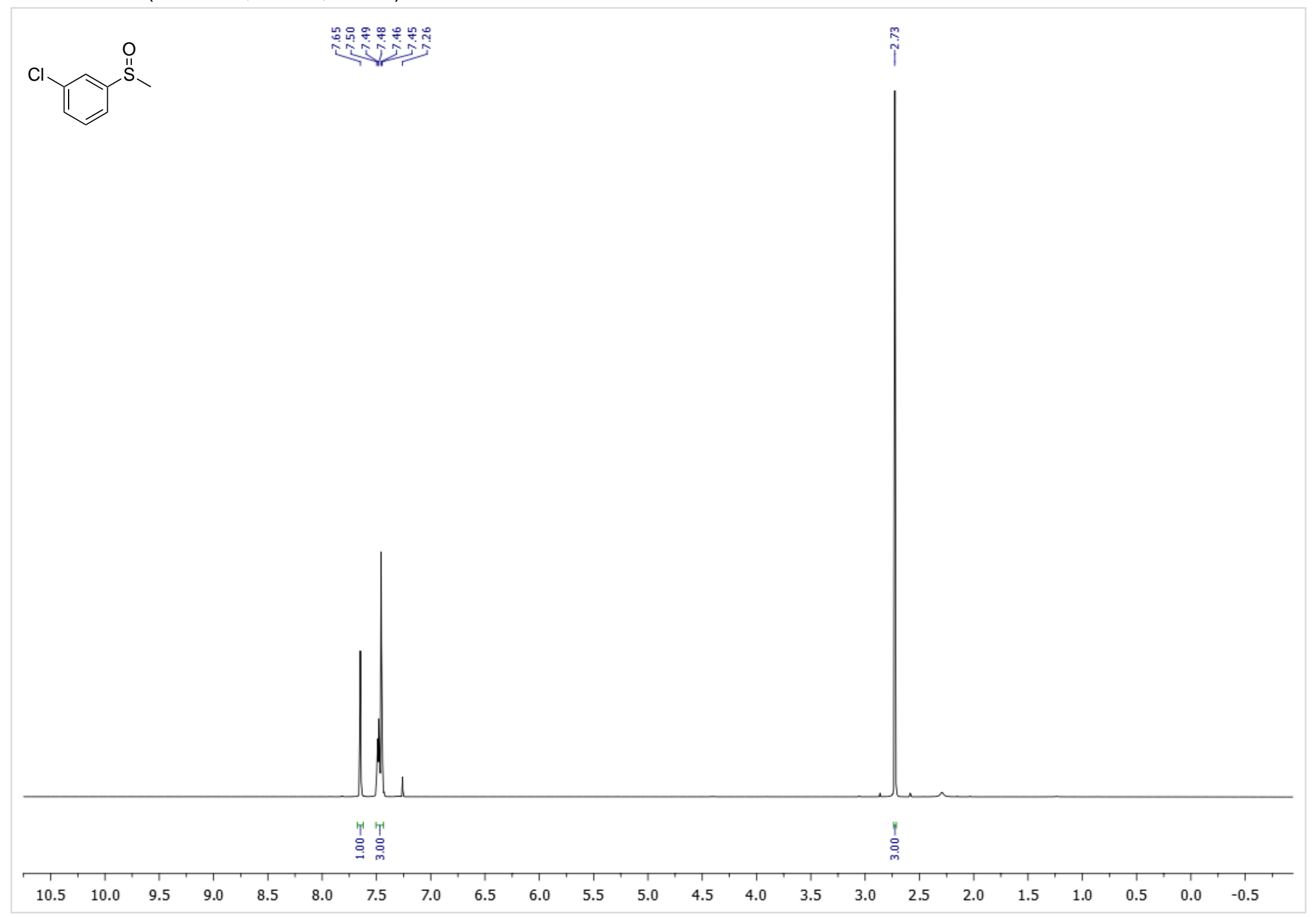


${ }^{13} \mathrm{C}\left\{{ }^{1} \mathrm{H}\right\}$ NMR $\left(126 \mathrm{MHz}, \mathrm{CDCl}_{3}, 298 \mathrm{~K}\right)$

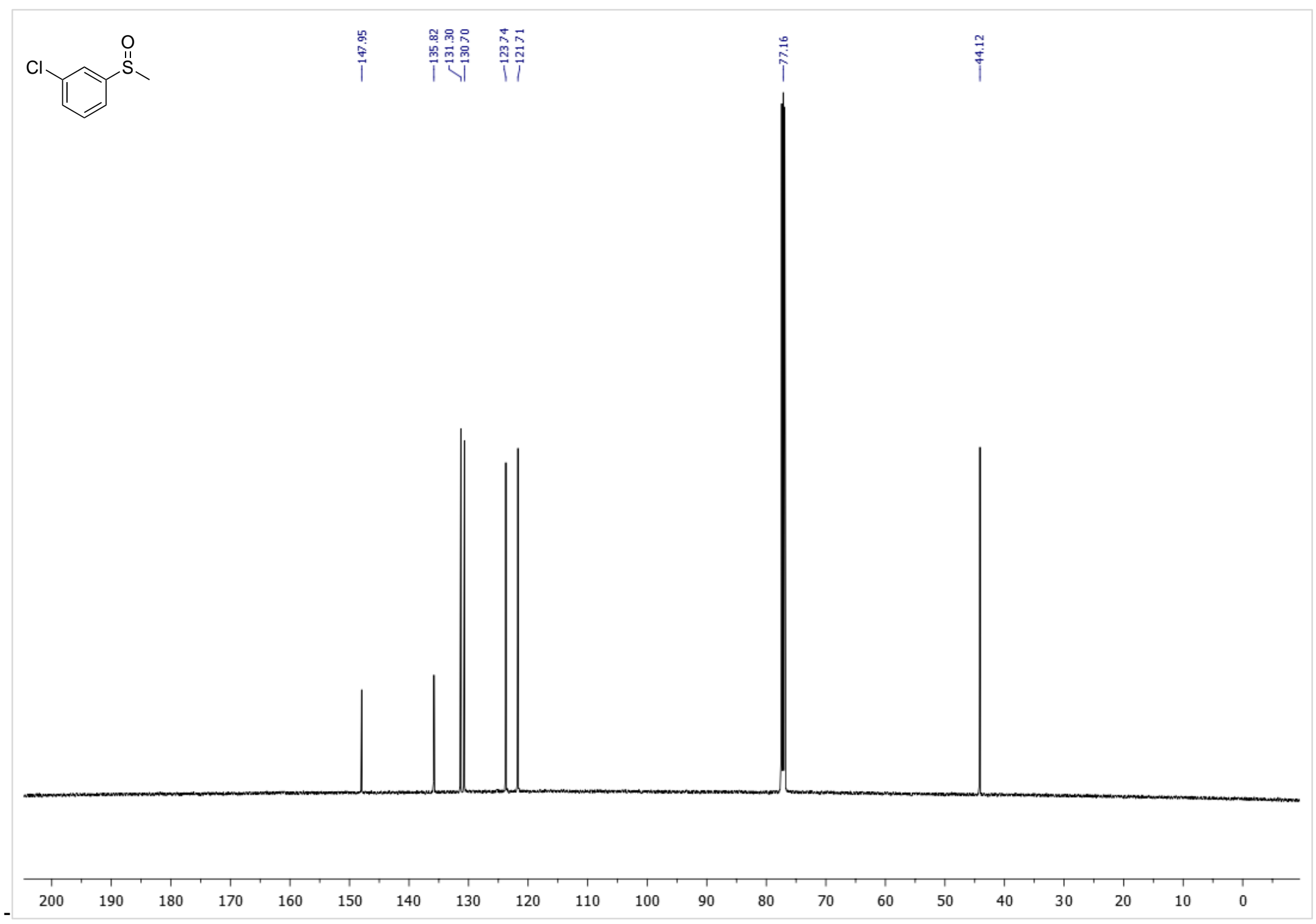

S35 
1-bromo-2-(methylsulfinyl)benzene (2m)

${ }^{1} \mathrm{H}$ NMR $\left(500 \mathrm{MHz}, \mathrm{CDCl}_{3}, 298 \mathrm{~K}\right)$

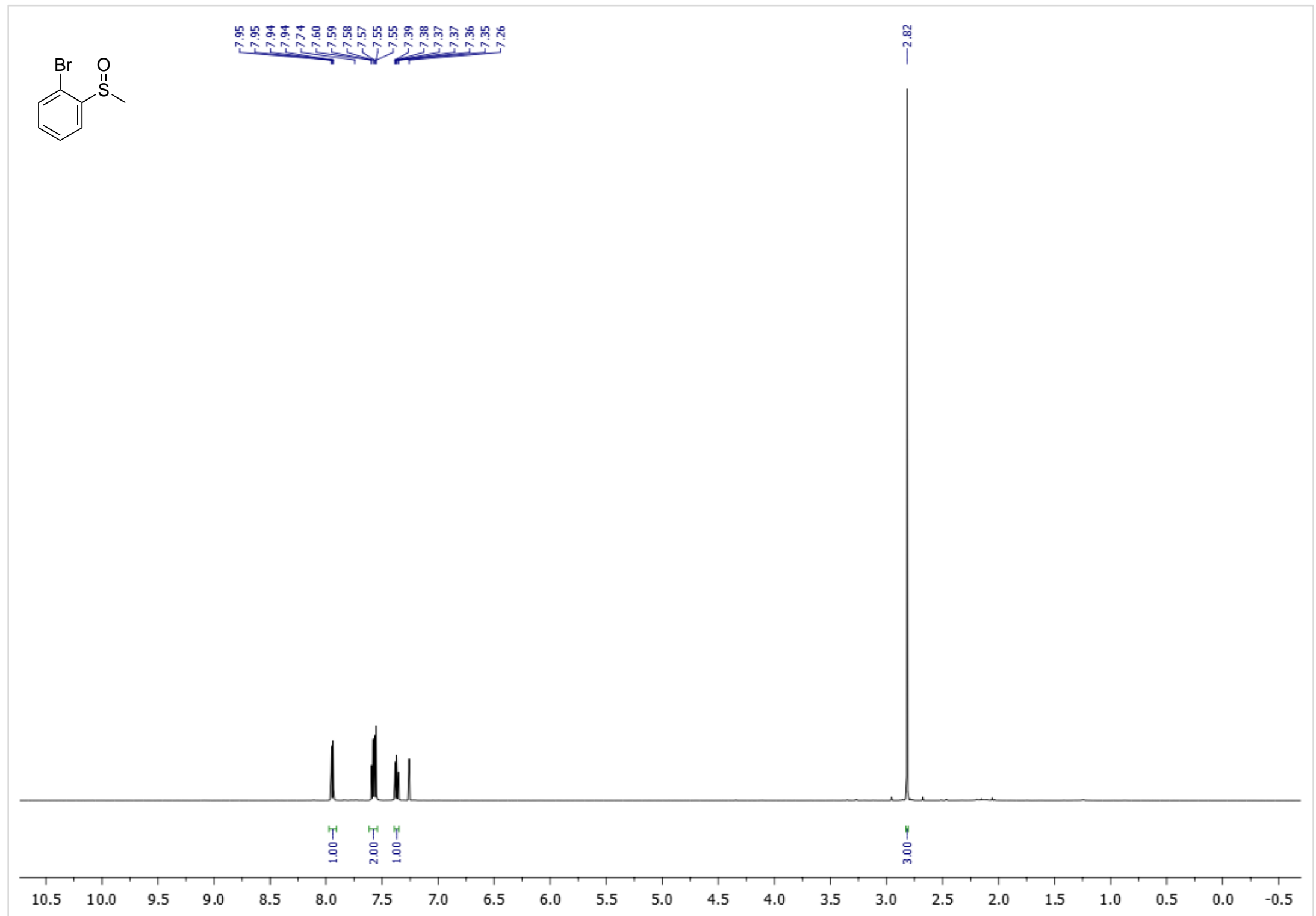


${ }^{13} \mathrm{C}\left\{{ }^{1} \mathrm{H}\right\}$ NMR $\left(126 \mathrm{MHz}, \mathrm{CDCl}_{3}, 298 \mathrm{~K}\right)$

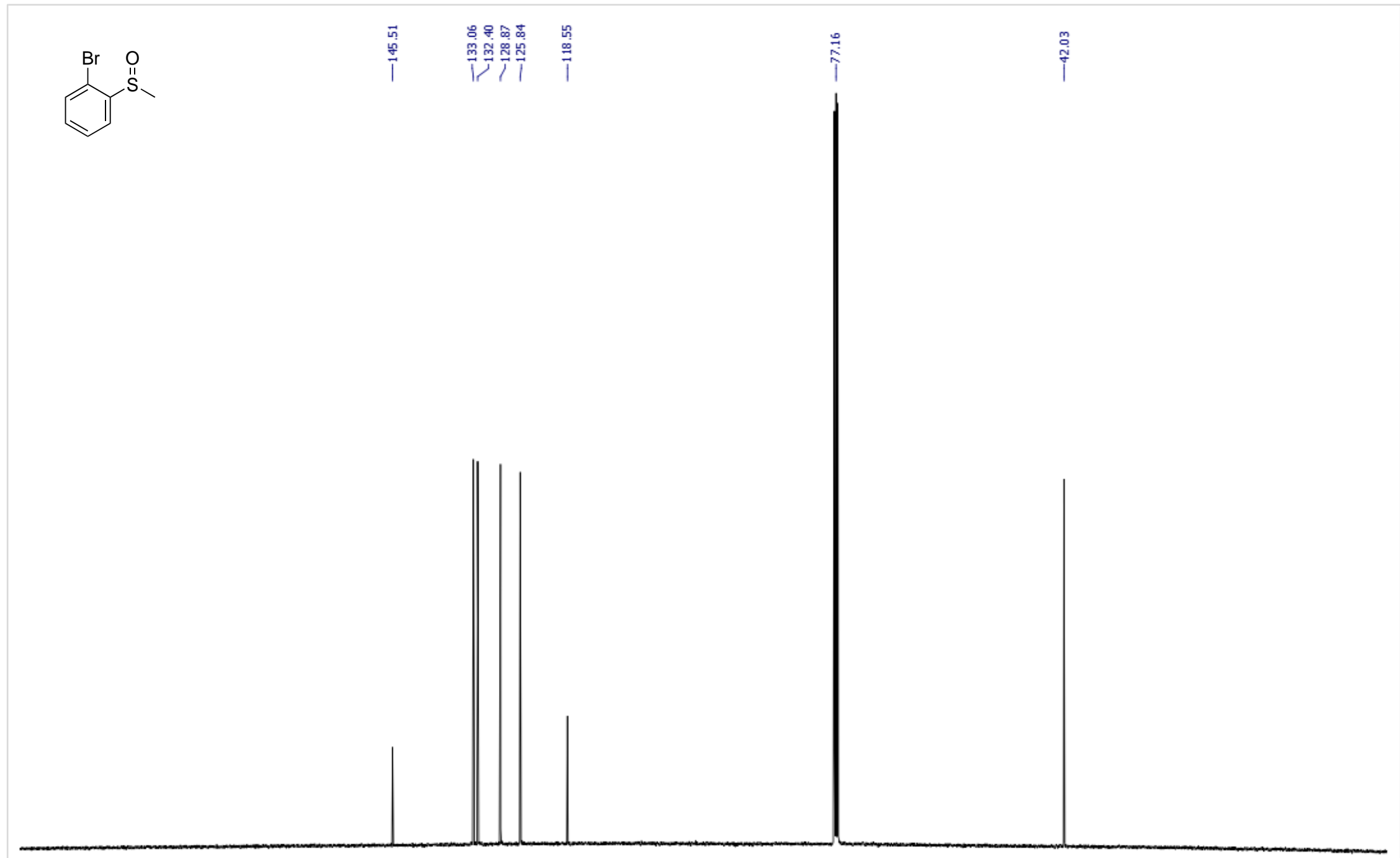

$$
200
$$


1-fluoro-2-(methylsulfinyl)benzene (2n)

${ }^{1} \mathbf{H}$ NMR $\left(500 \mathrm{MHz}, \mathrm{CDCl}_{3}, 298 \mathrm{~K}\right)$

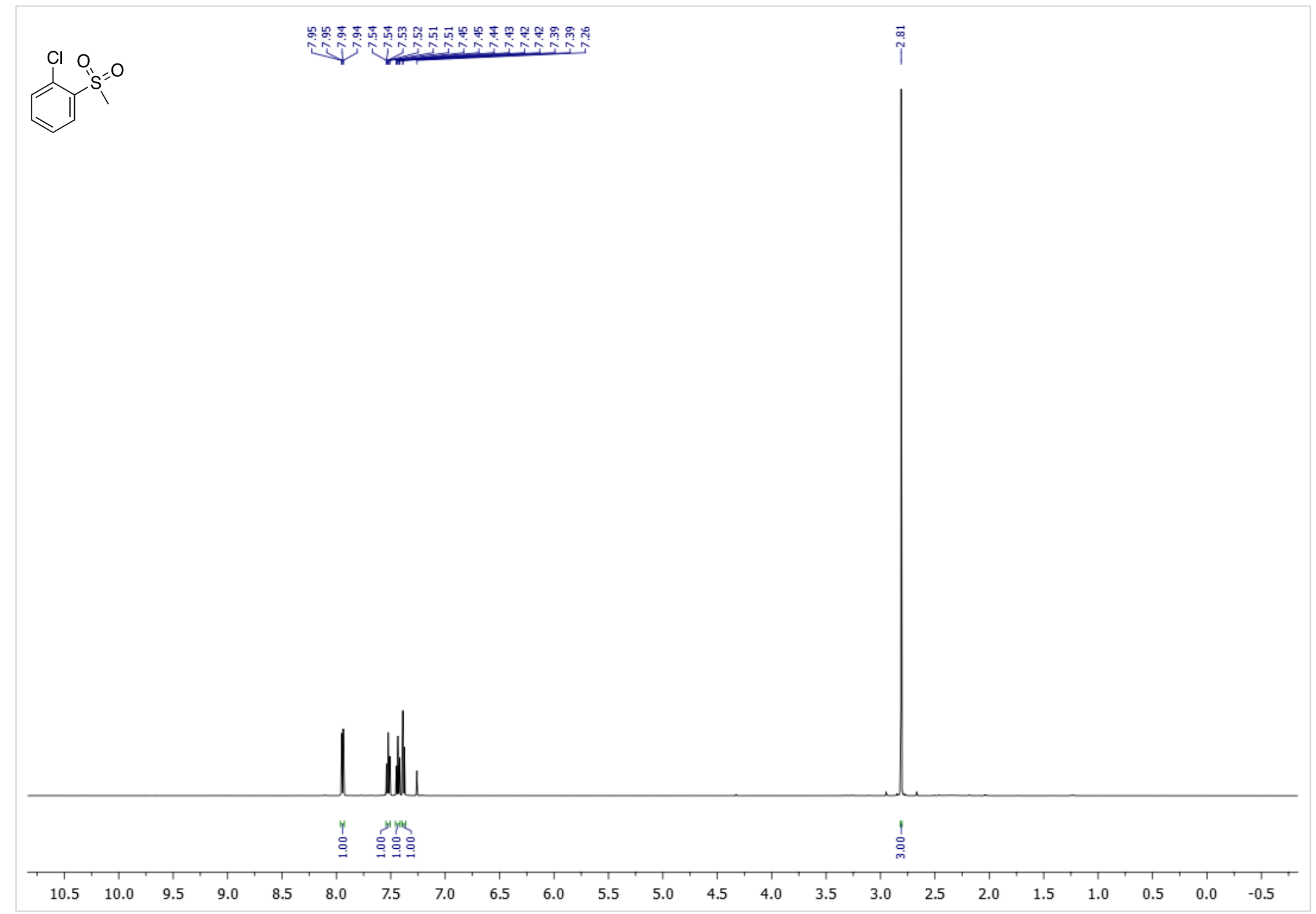

S38 
${ }^{13} \mathrm{C}\left\{{ }^{1} \mathrm{H}\right\}$ NMR $\left(126 \mathrm{MHz}, \mathrm{CDCl}_{3}, 298 \mathrm{~K}\right)$

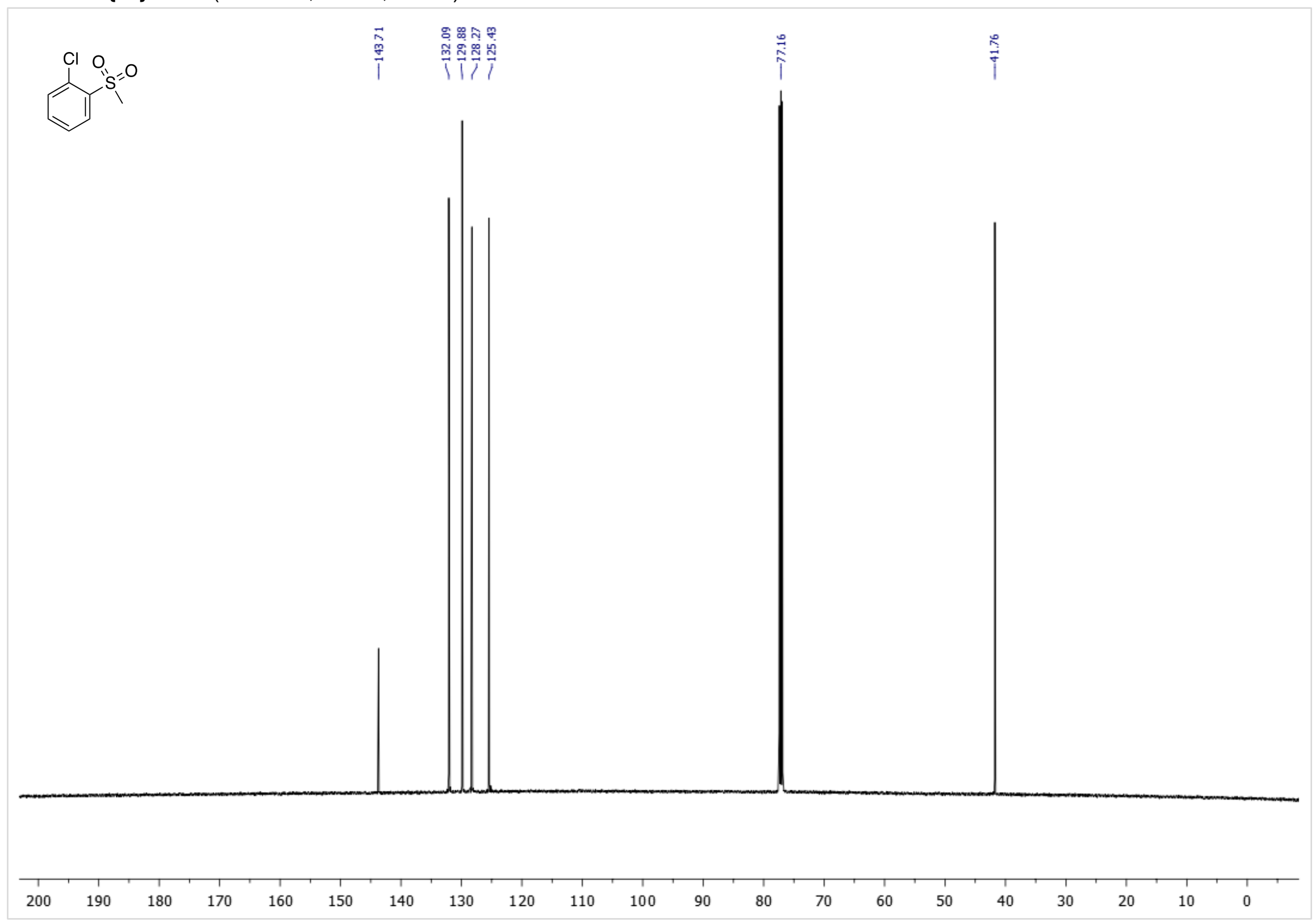


1-iodo-2-(methylsulfinyl)benzene (2o)

${ }^{1} \mathbf{H}$ NMR $\left(500 \mathrm{MHz}, \mathrm{CDCl}_{3}, 298 \mathrm{~K}\right)$

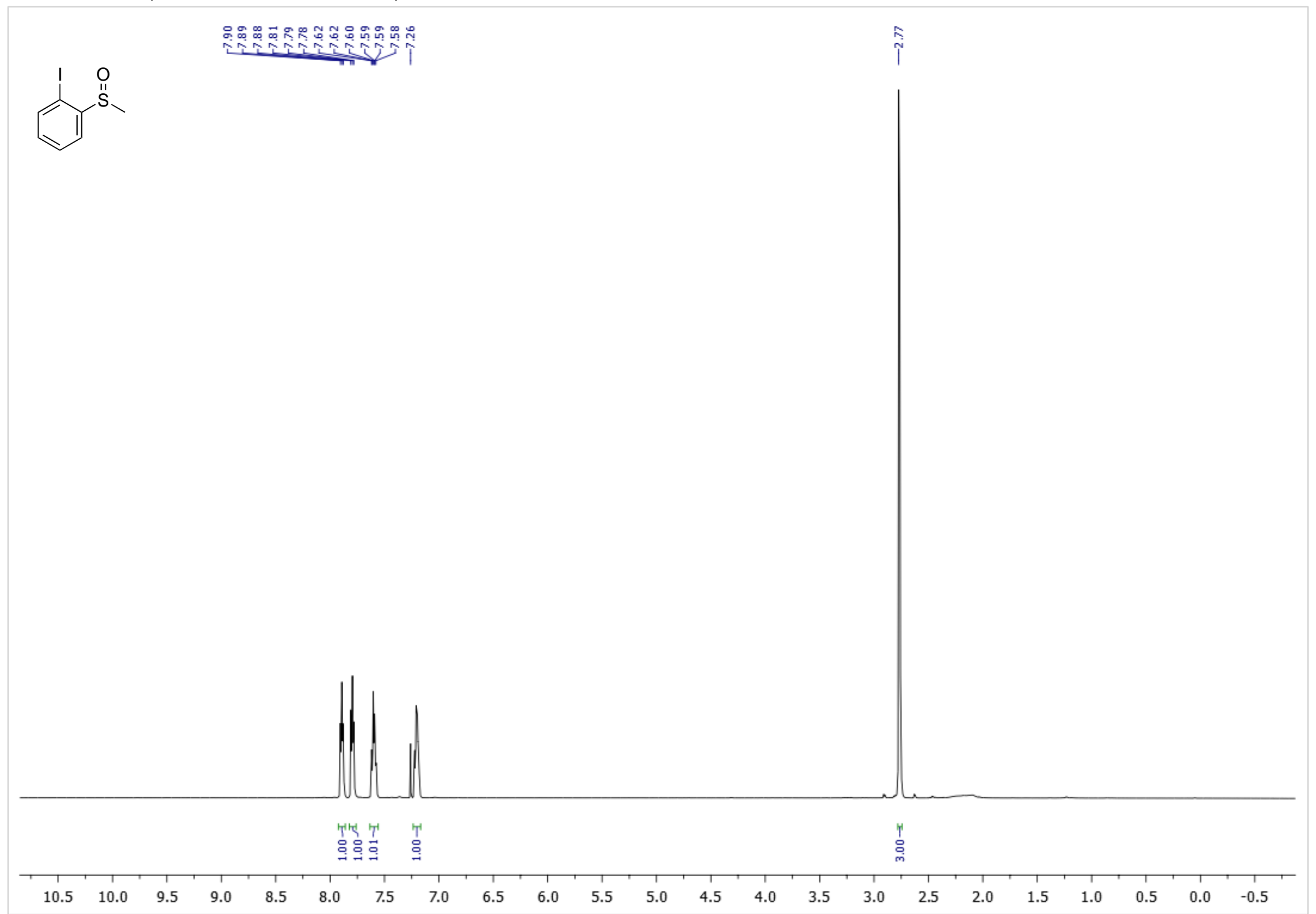


${ }^{13} \mathrm{C}\left\{{ }^{1} \mathrm{H}\right\}$ NMR $\left(126 \mathrm{MHz}, \mathrm{CDCl}_{3}, 298 \mathrm{~K}\right)$

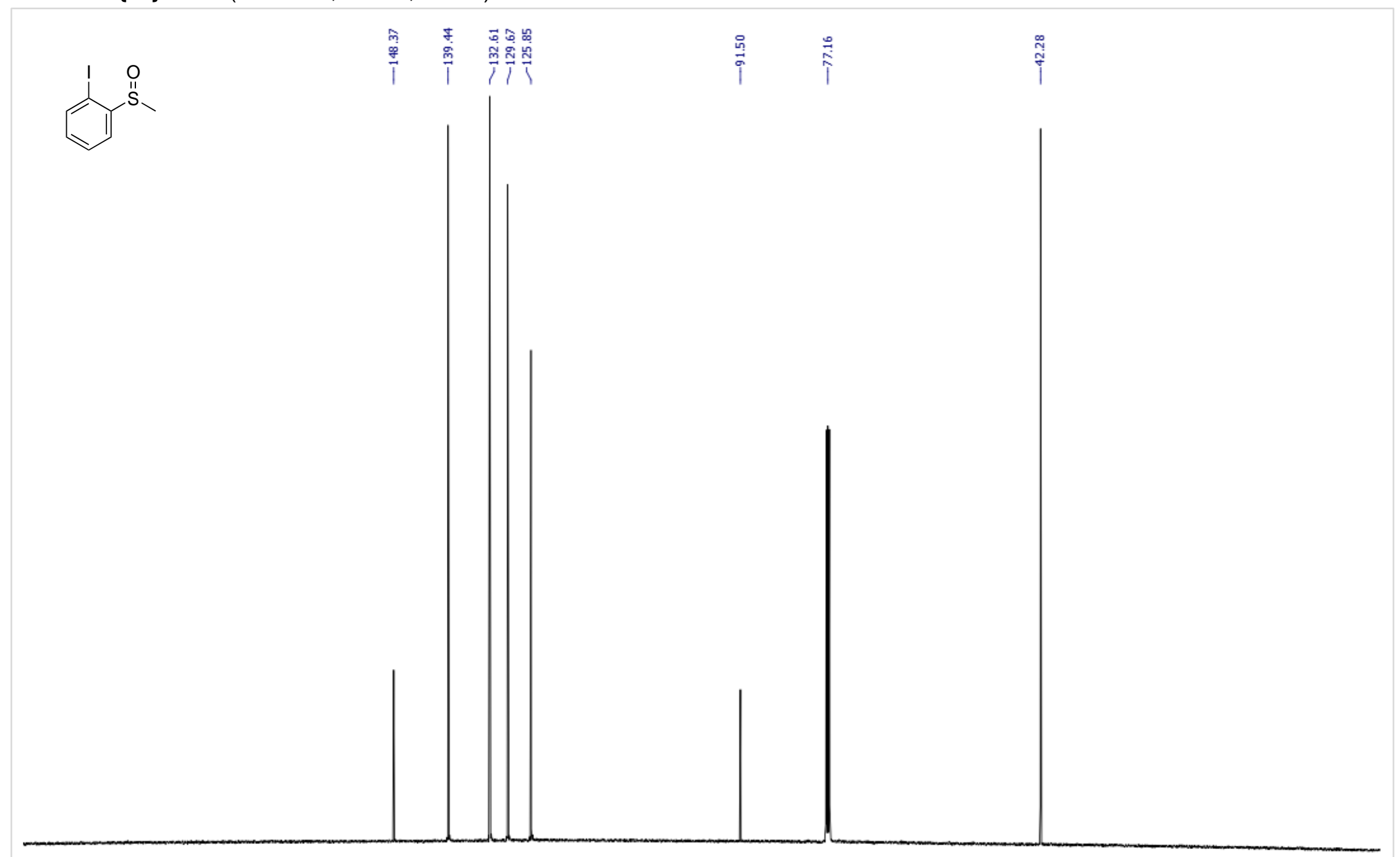


1,3-dichloro-5-(methylsulfinyl)benzene (2p)

${ }^{1} \mathrm{H}$ NMR $\left(500 \mathrm{MHz}, \mathrm{CDCl}_{3}, 298 \mathrm{~K}\right)$

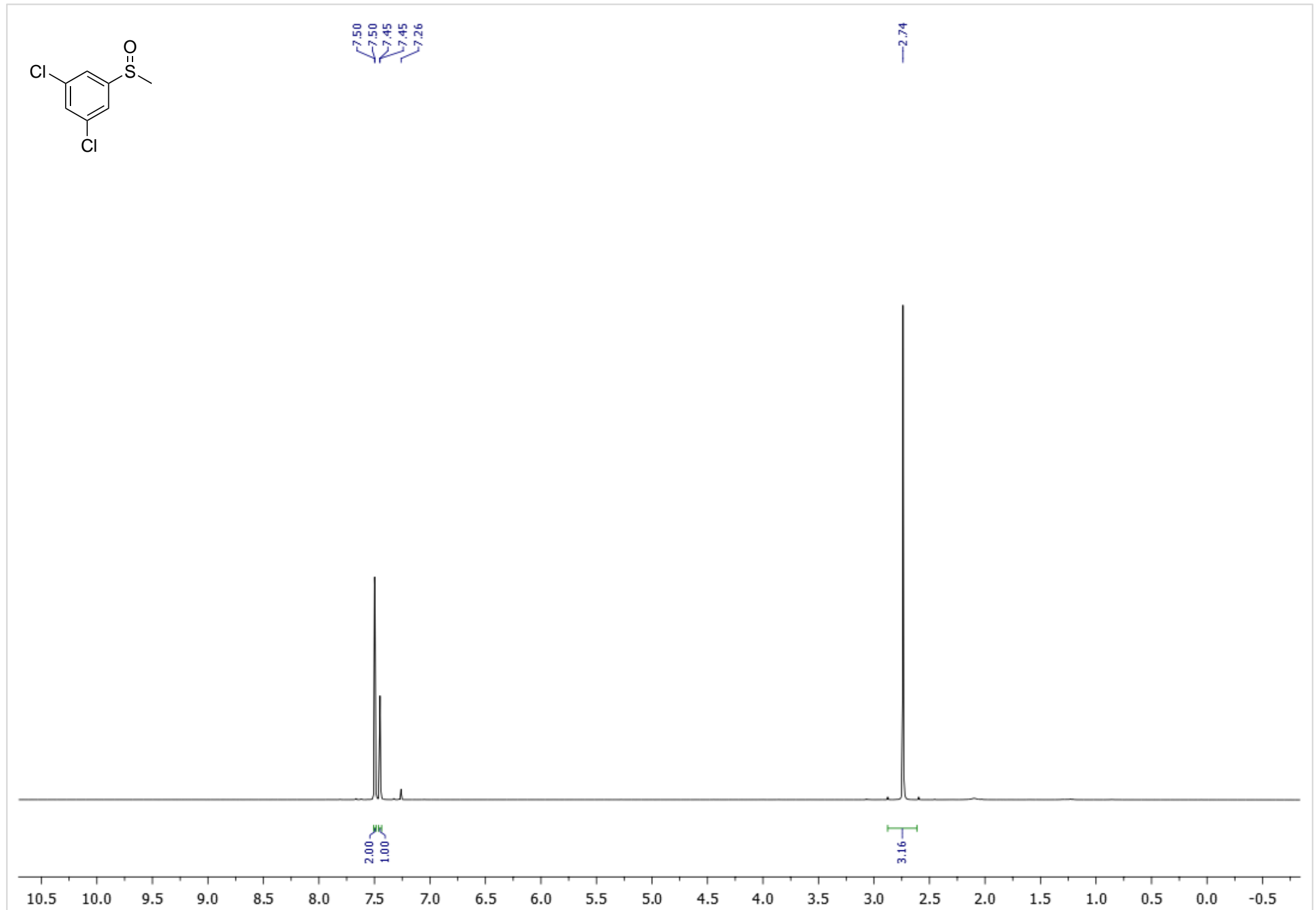


${ }^{13} \mathbf{C}\left\{{ }^{1} \mathrm{H}\right\}$ NMR $\left(126 \mathrm{MHz}, \mathrm{CDCl}_{3}, 298 \mathrm{~K}\right)$

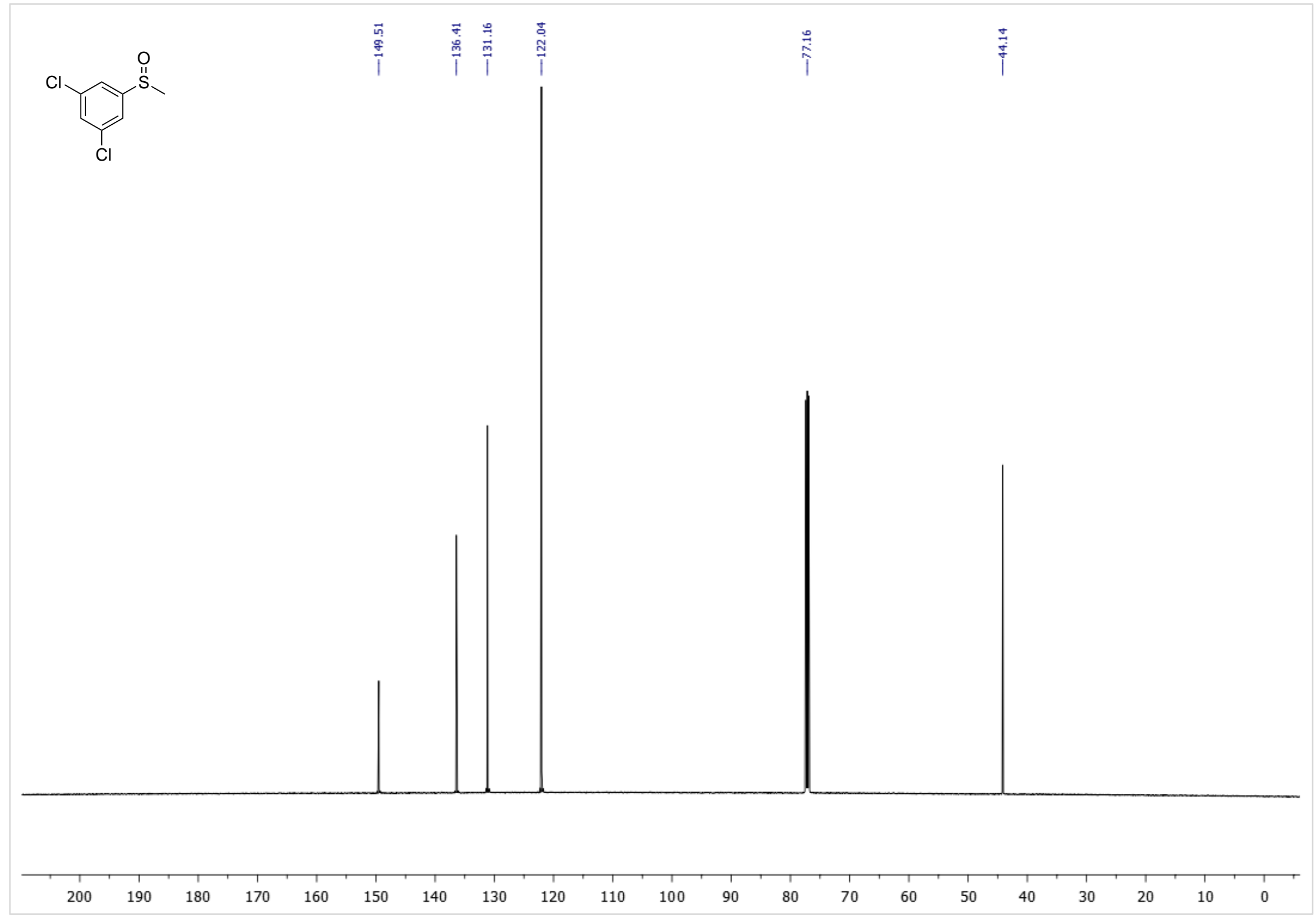

S43 
${ }^{1} \mathbf{H}$ NMR (500 MHz, $\left.\mathrm{CDCl}_{3}, 298 \mathrm{~K}\right)$

(ethylsulfinyl)benzene (2q)

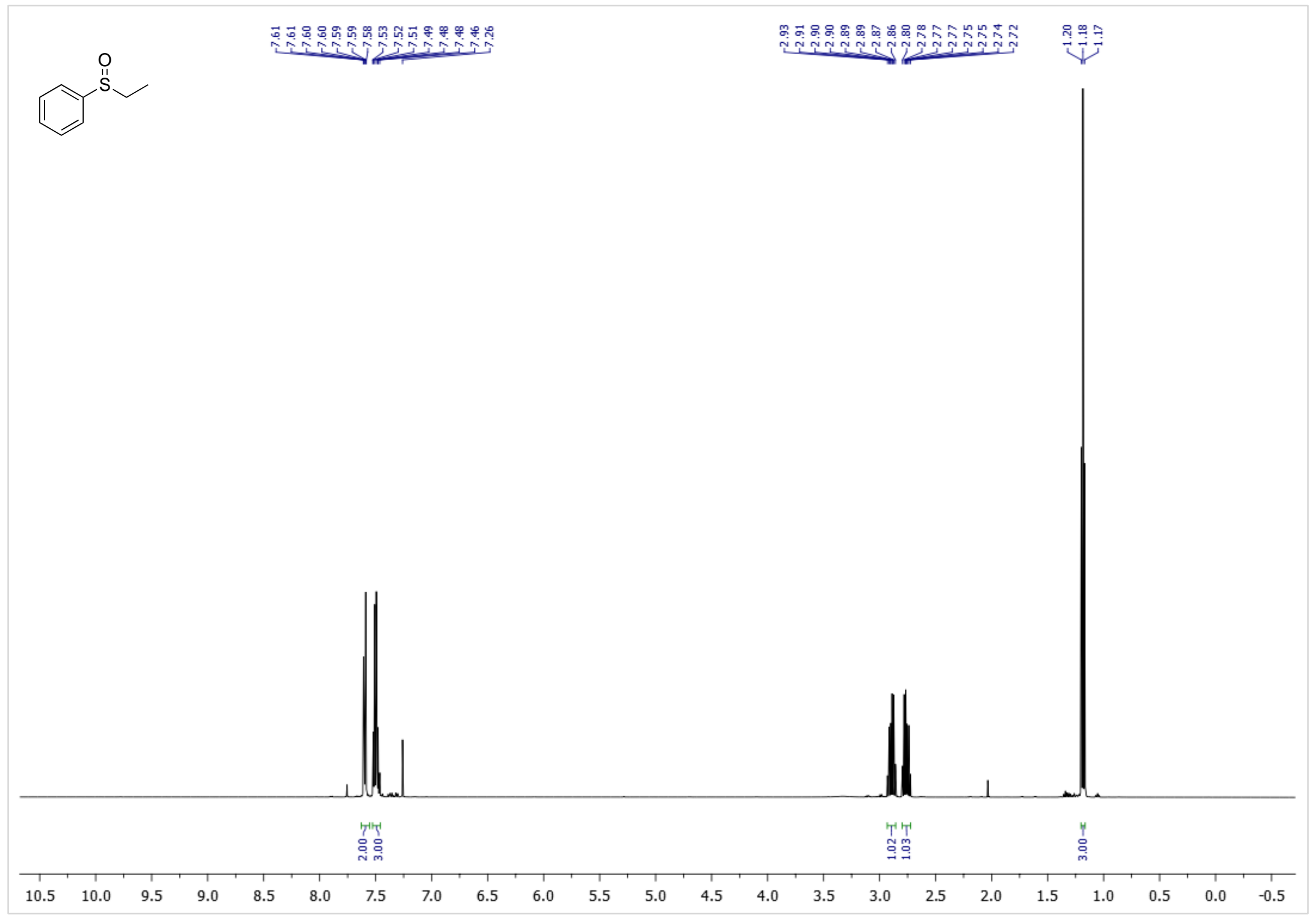


${ }^{13} \mathbf{C}\left\{{ }^{1} \mathrm{H}\right\}$ NMR $\left(126 \mathrm{MHz}, \mathrm{CDCl}_{3}, 298 \mathrm{~K}\right)$

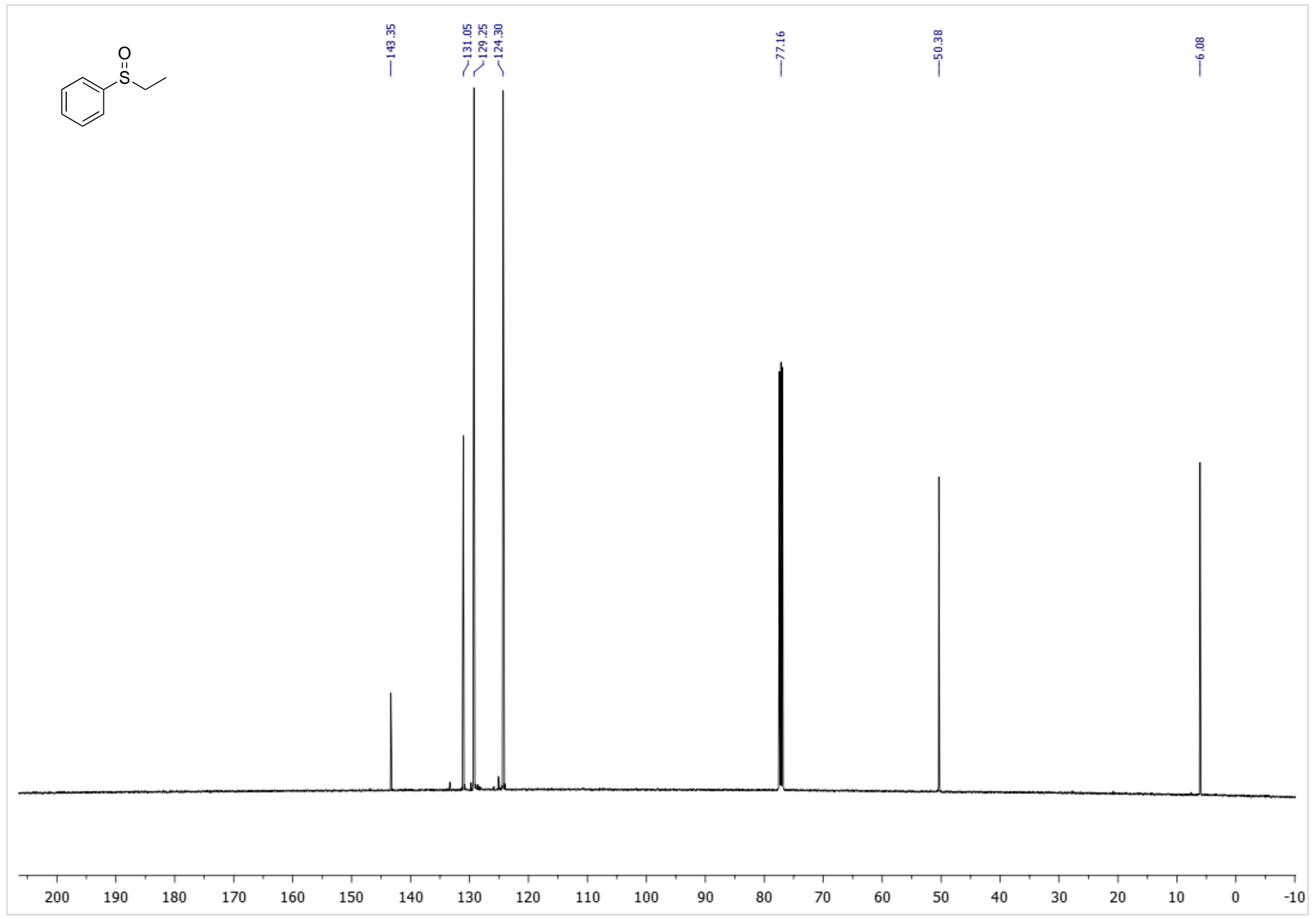

S45 
${ }^{1} \mathbf{H}$ NMR $\left(500 \mathrm{MHz}, \mathrm{CDCl}_{3}, 298 \mathrm{~K}\right)$

propylsulfinyl)benzene (2r)

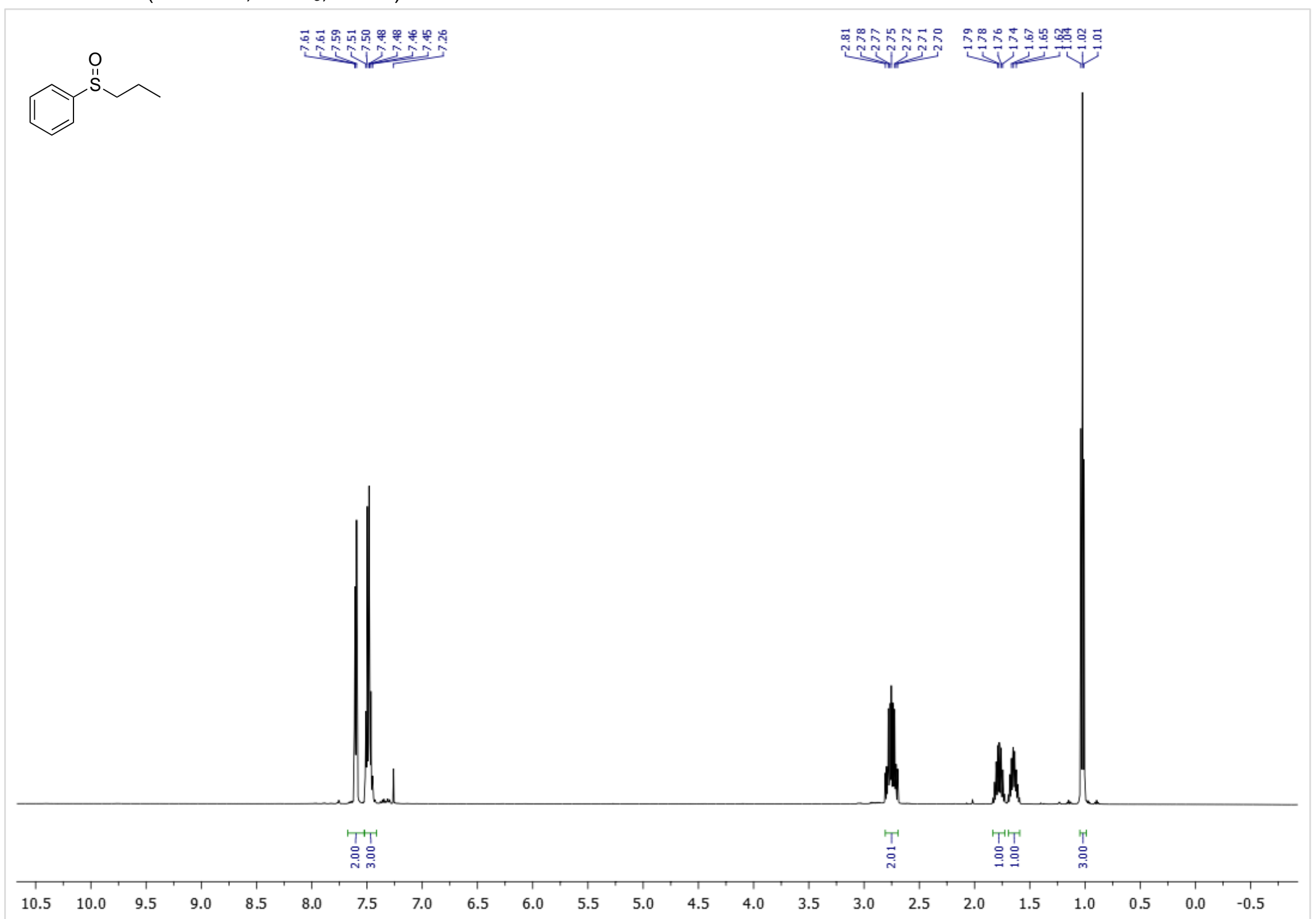


${ }^{13} \mathbf{C}\left\{{ }^{1} \mathrm{H}\right\}$ NMR $\left(126 \mathrm{MHz}, \mathrm{CDCl}_{3}, 298 \mathrm{~K}\right)$

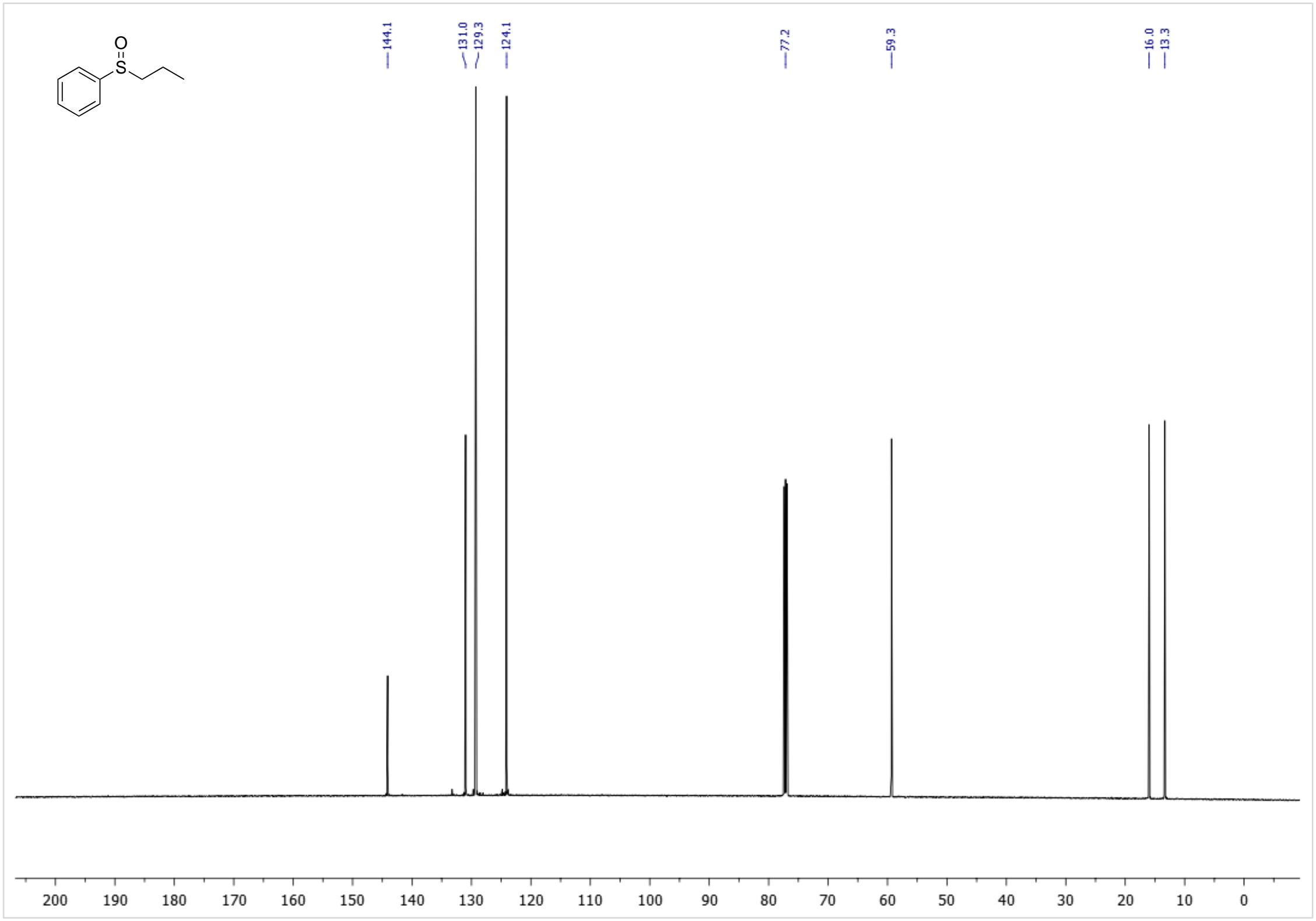

S47 
${ }^{1} \mathbf{H}$ NMR (500 MHz, $\left.\mathrm{CDCl}_{3}, 298 \mathrm{~K}\right)$

(cyclopropylsulfinyl)benzene (2s)

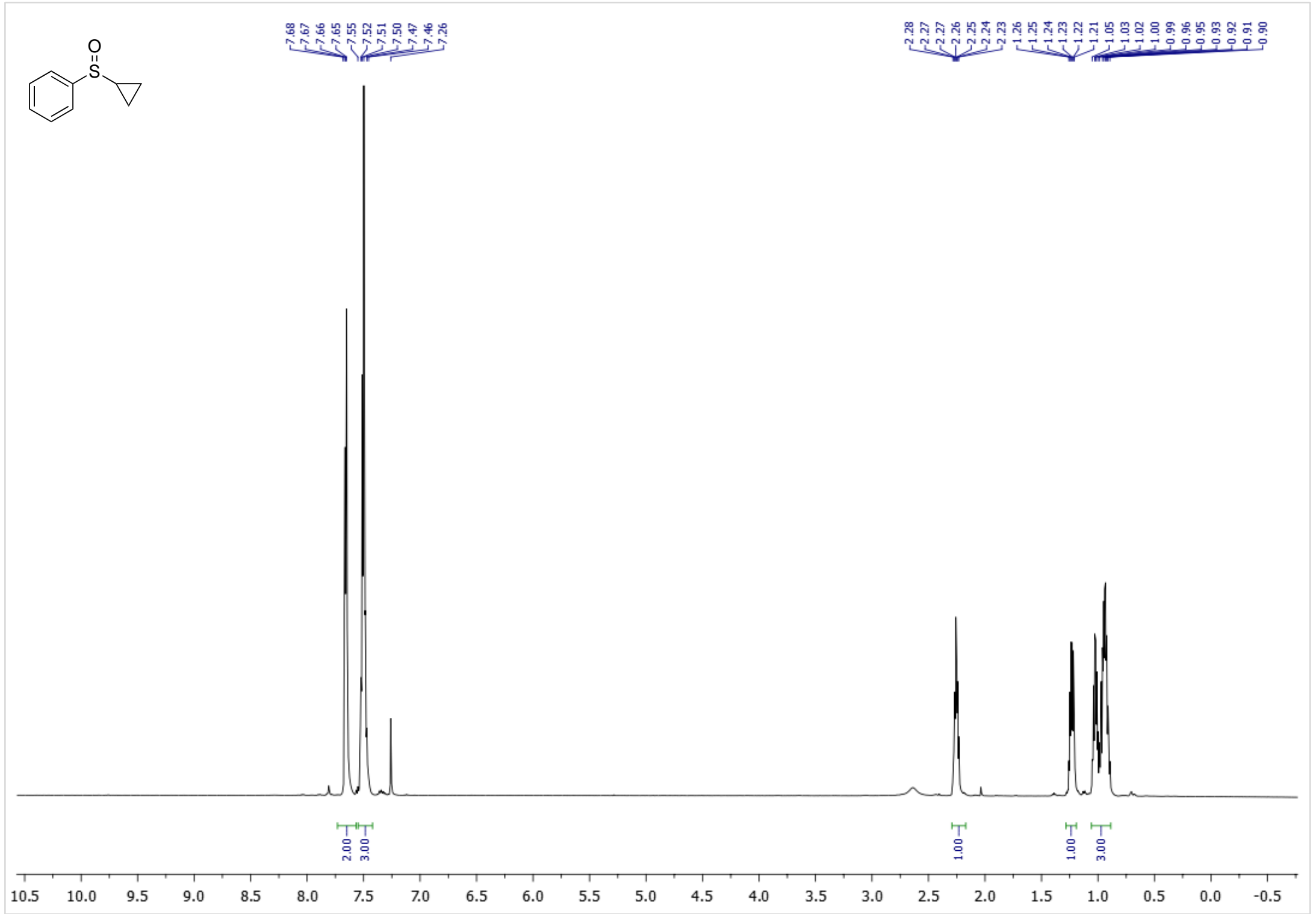


${ }^{13} \mathrm{C}\left\{{ }^{1} \mathrm{H}\right\}$ NMR $\left(126 \mathrm{MHz}, \mathrm{CDCl}_{3}, 298 \mathrm{~K}\right)$

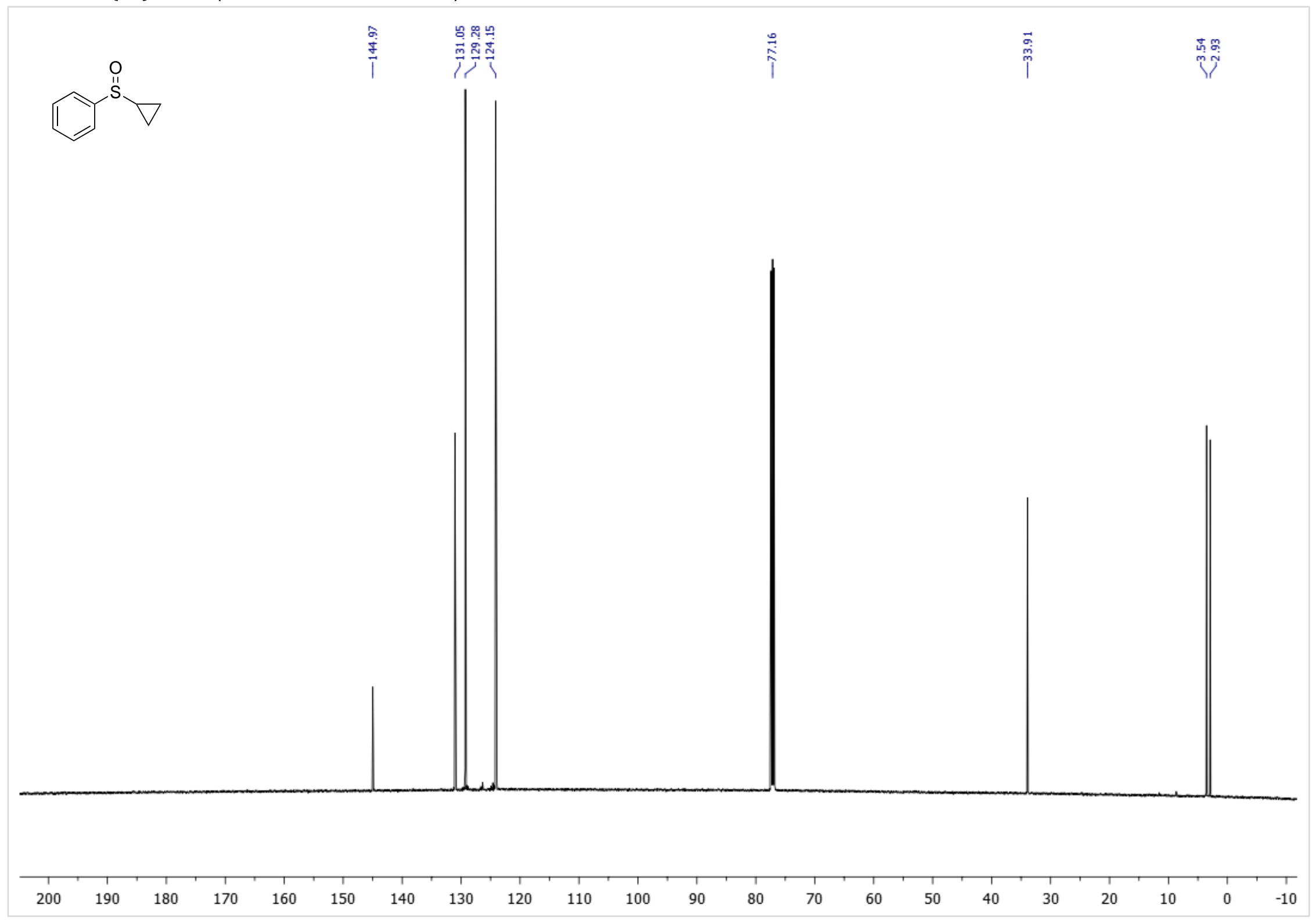

S49 
${ }^{\mathbf{1}} \mathbf{H}$ NMR $\left(500 \mathrm{MHz}, \mathrm{CDCl}_{3}, 298 \mathrm{~K}\right)$

(allylsulfinyl)benzene (2t)

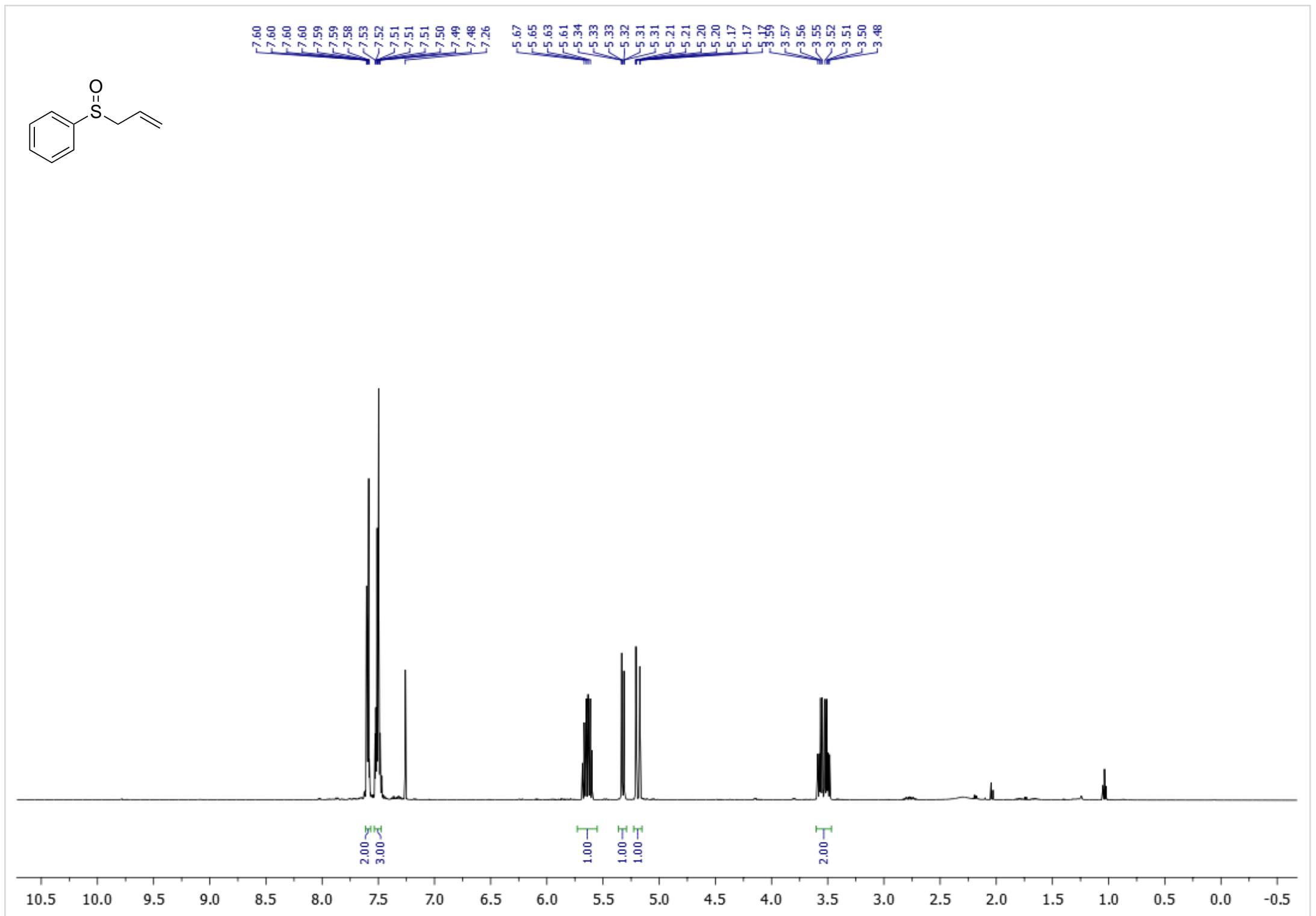


${ }^{13} \mathrm{C}\left\{{ }^{1} \mathrm{H}\right\}$ NMR $\left(126 \mathrm{MHz}, \mathrm{CDCl}_{3}, 298 \mathrm{~K}\right)$

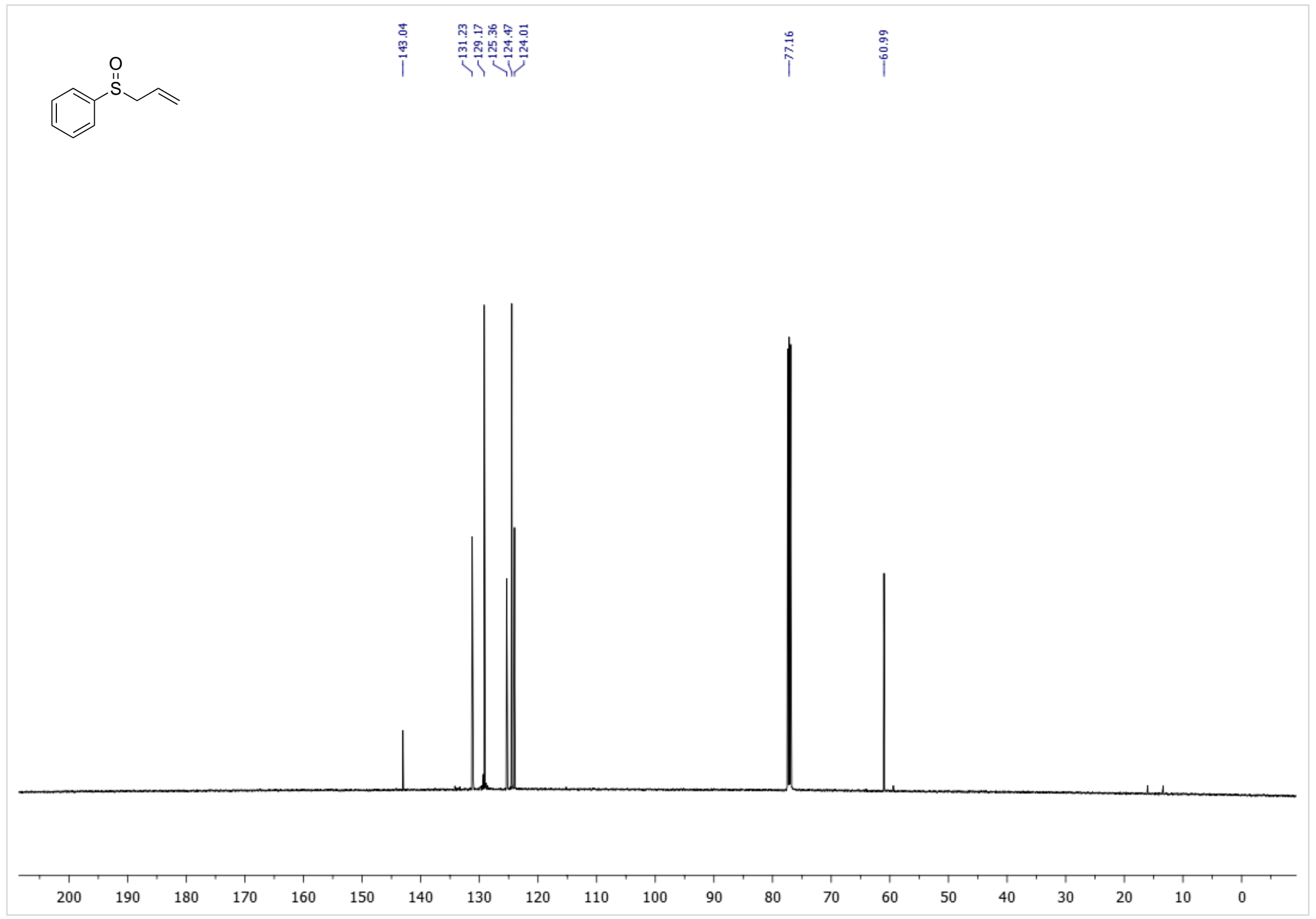

S51 
${ }^{\mathbf{1}} \mathbf{H}$ NMR $\left(500 \mathrm{MHz}, \mathrm{CDCl}_{3}, 298 \mathrm{~K}\right)$

(benzylsulfinyl)benzene (2u)

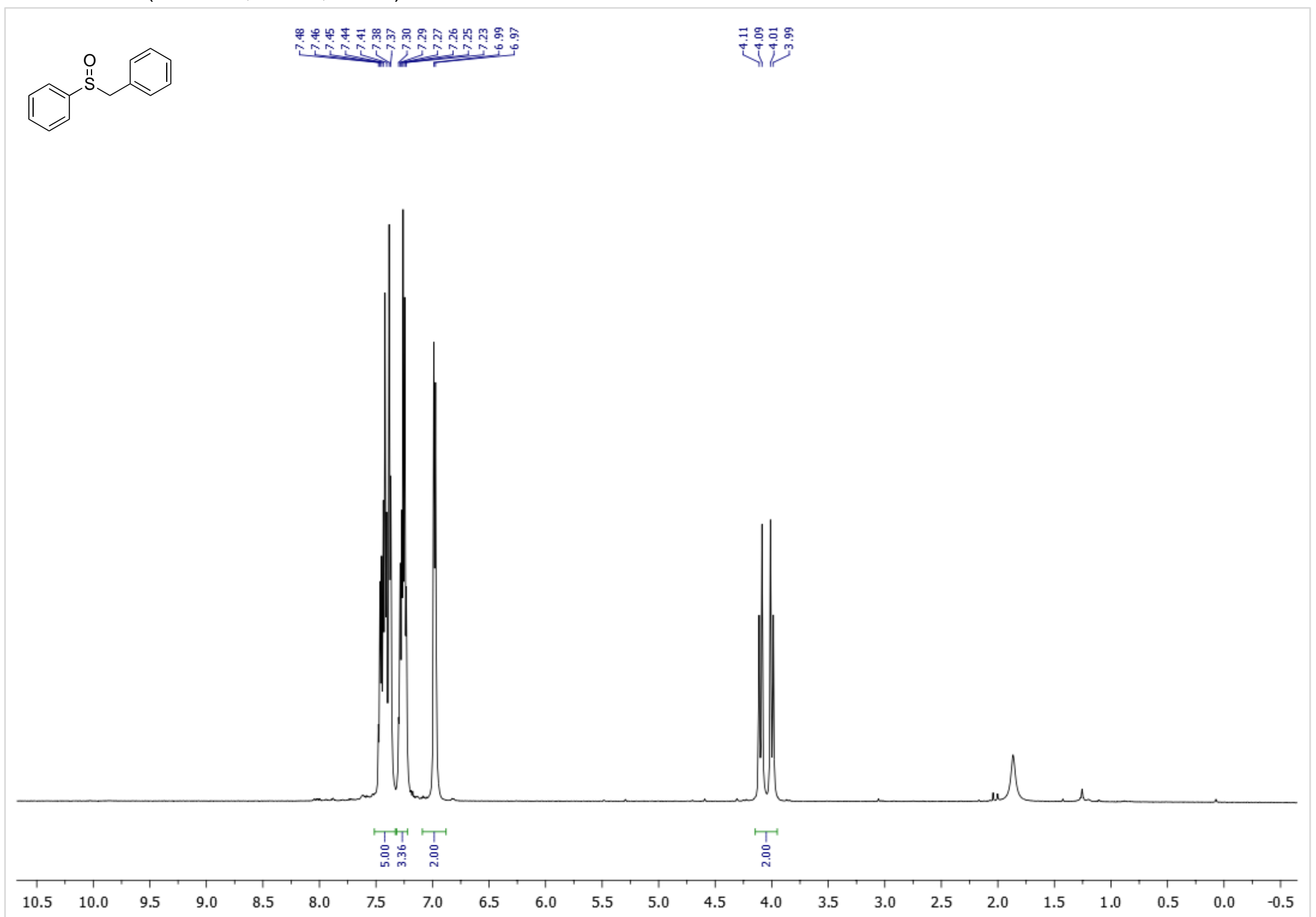


${ }^{13} \mathrm{C}\left\{{ }^{1} \mathrm{H}\right\}$ NMR $\left(126 \mathrm{MHz}, \mathrm{CDCl}_{3}, 298 \mathrm{~K}\right)$

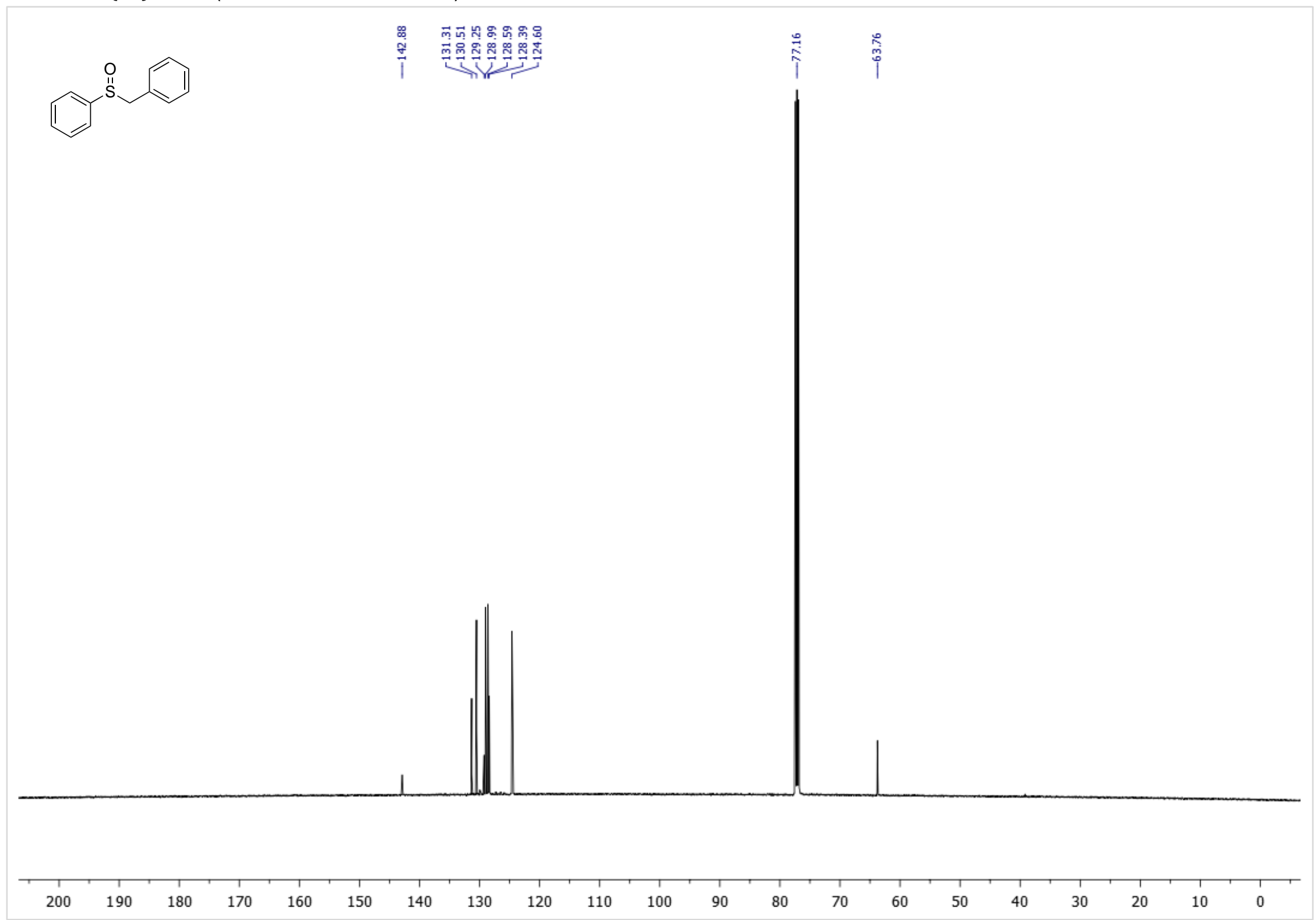


${ }^{1} \mathbf{H}$ NMR (500 MHz, $\left.\mathrm{CDCl}_{3}, 298 \mathrm{~K}\right)$

sulfinyldibenzene (2v)

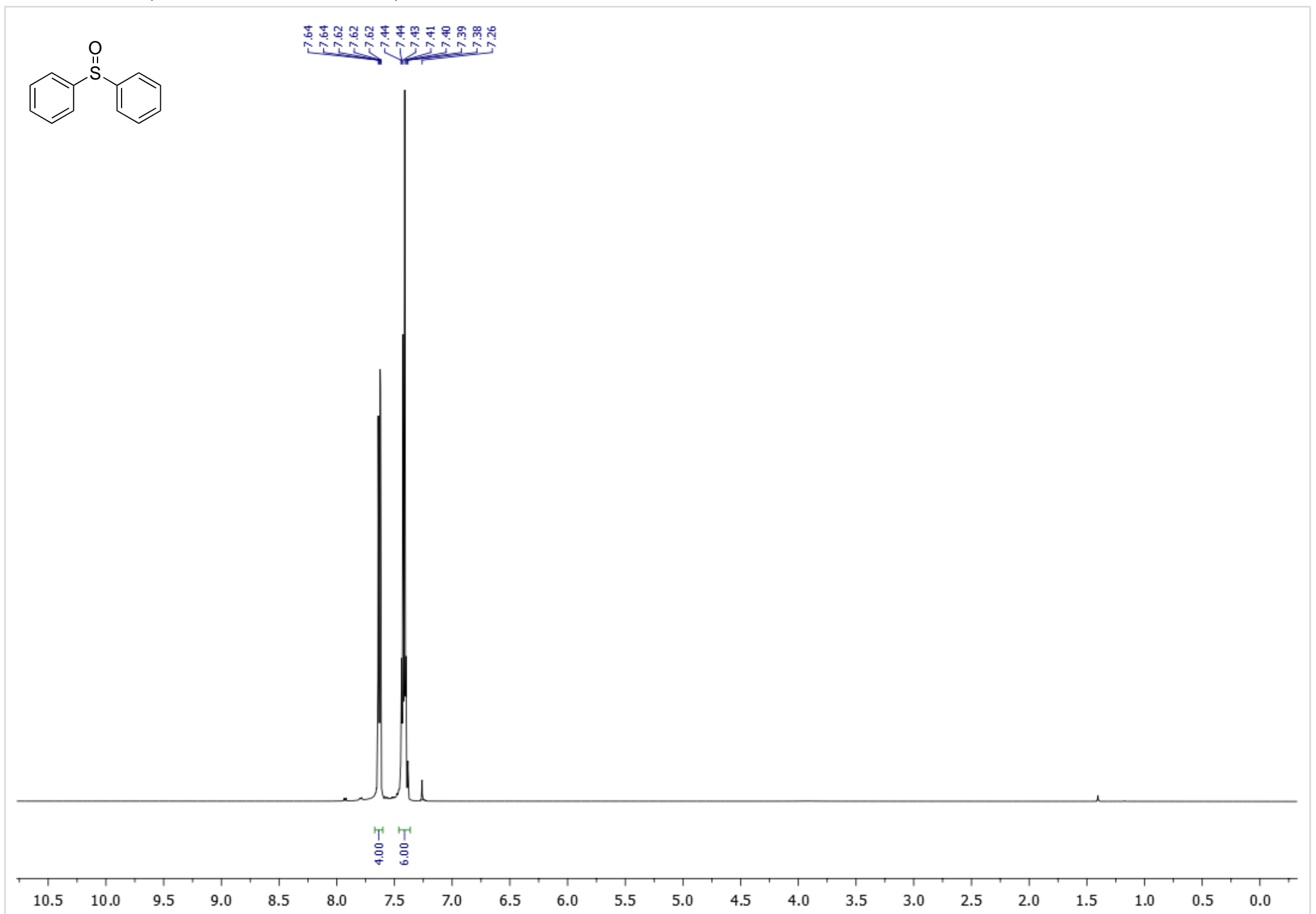


${ }^{13} \mathrm{C}\left\{{ }^{1} \mathrm{H}\right\}$ NMR $\left(126 \mathrm{MHz}, \mathrm{CDCl}_{3}, 298 \mathrm{~K}\right)$

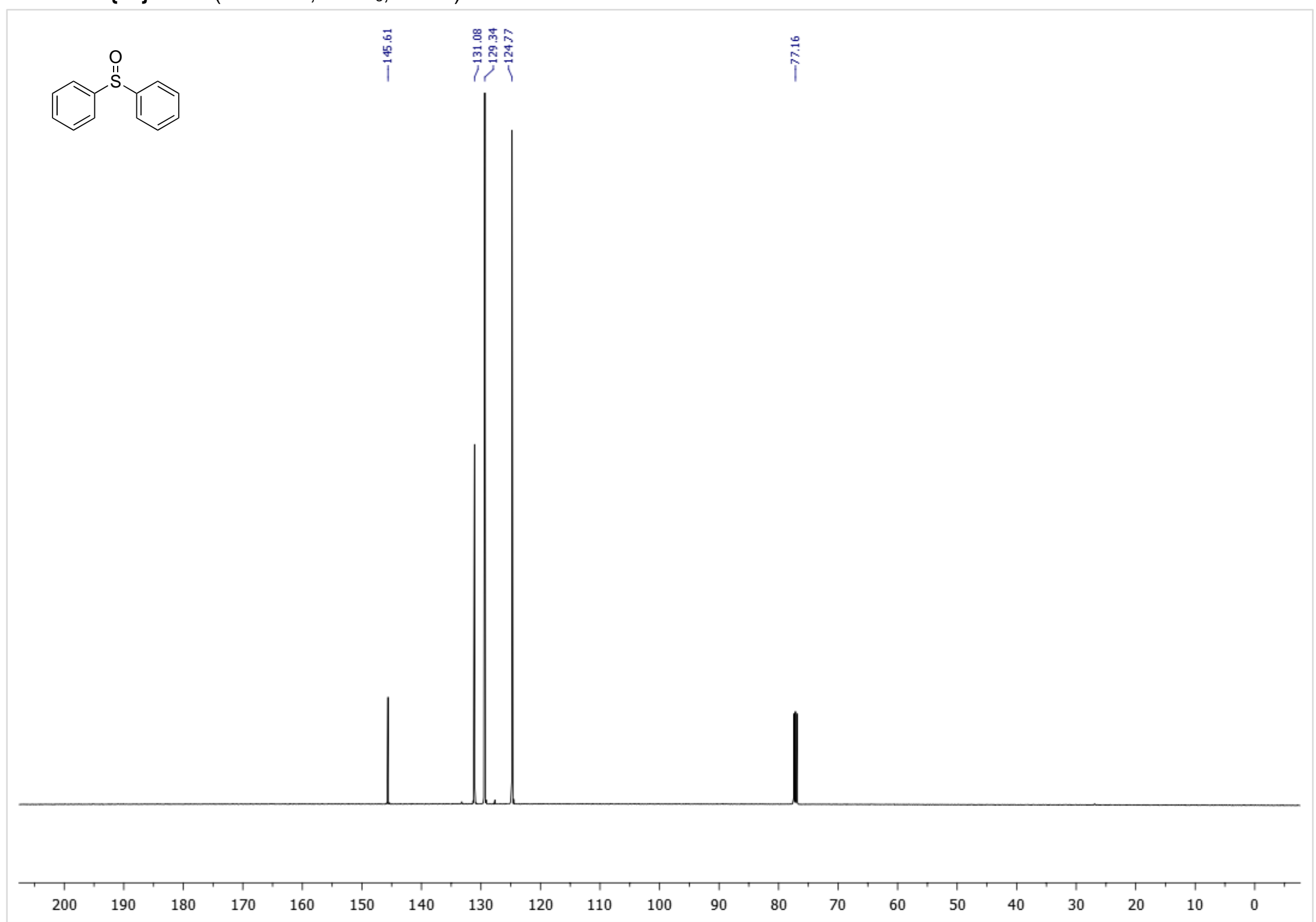


${ }^{1} \mathbf{H}$ NMR $\left(500 \mathrm{MHz}, \mathrm{CDCl}_{3}, 298 \mathrm{~K}\right)$

thiochroman-4-one 1-oxide (2w)

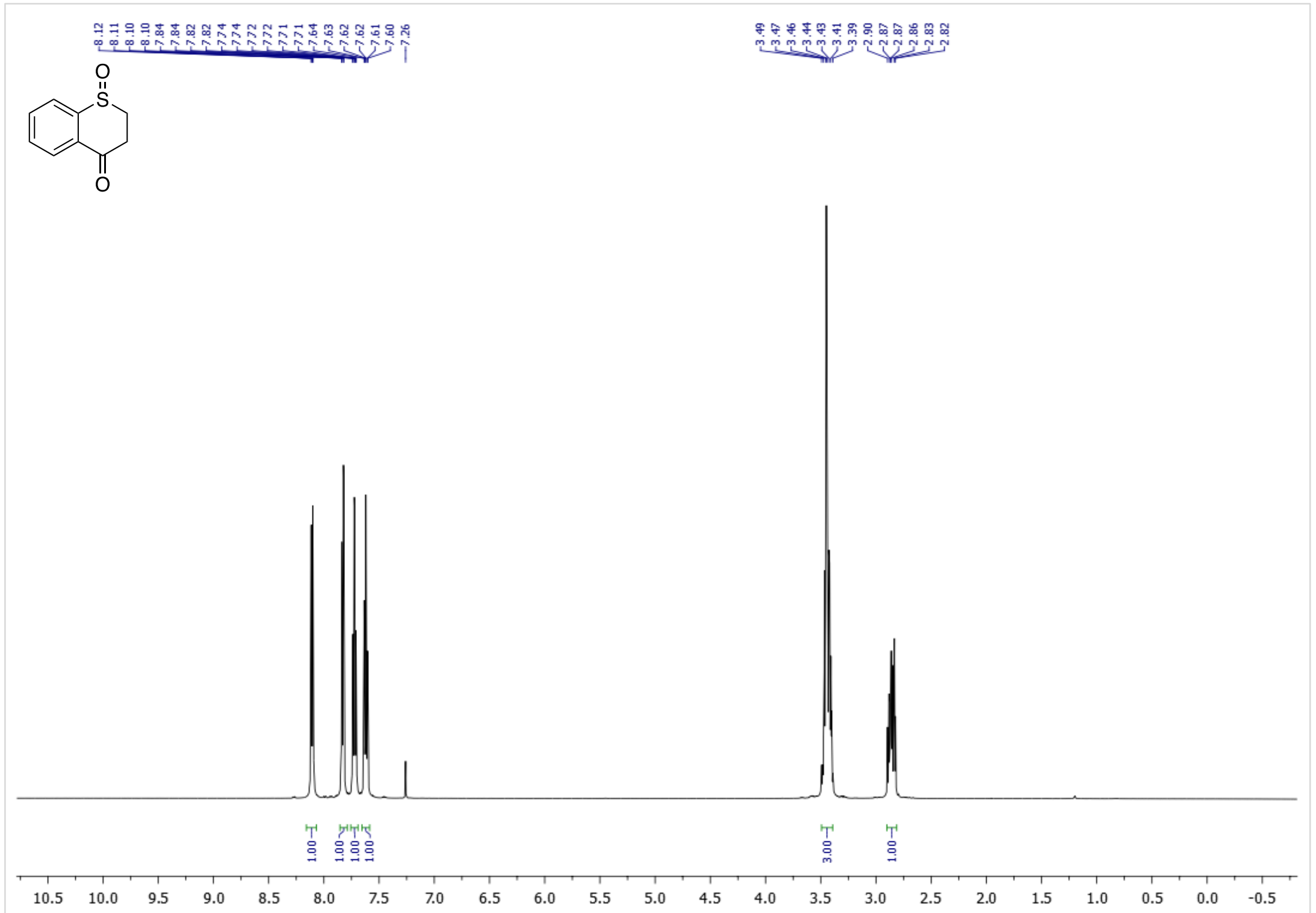


${ }^{13} \mathbf{C}\left\{{ }^{1} \mathrm{H}\right\}$ NMR $\left(126 \mathrm{MHz}, \mathrm{CDCl}_{3}, 298 \mathrm{~K}\right)$

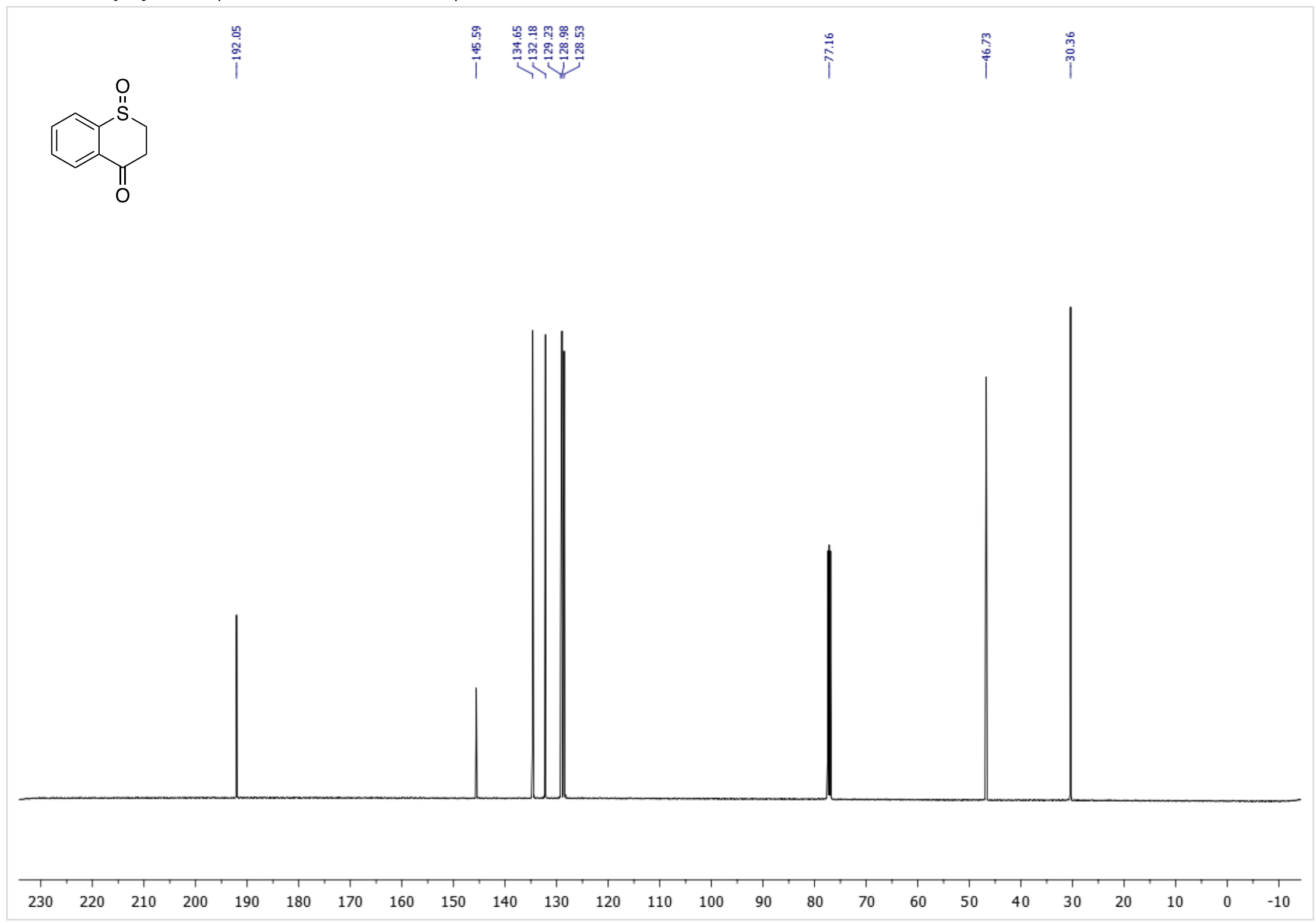

S57 
2-(methylsulfinyl)pyridine (2x)

${ }^{1} \mathbf{H}$ NMR (500 MHz, $\left.\mathrm{CDCl}_{3}, 298 \mathrm{~K}\right)$

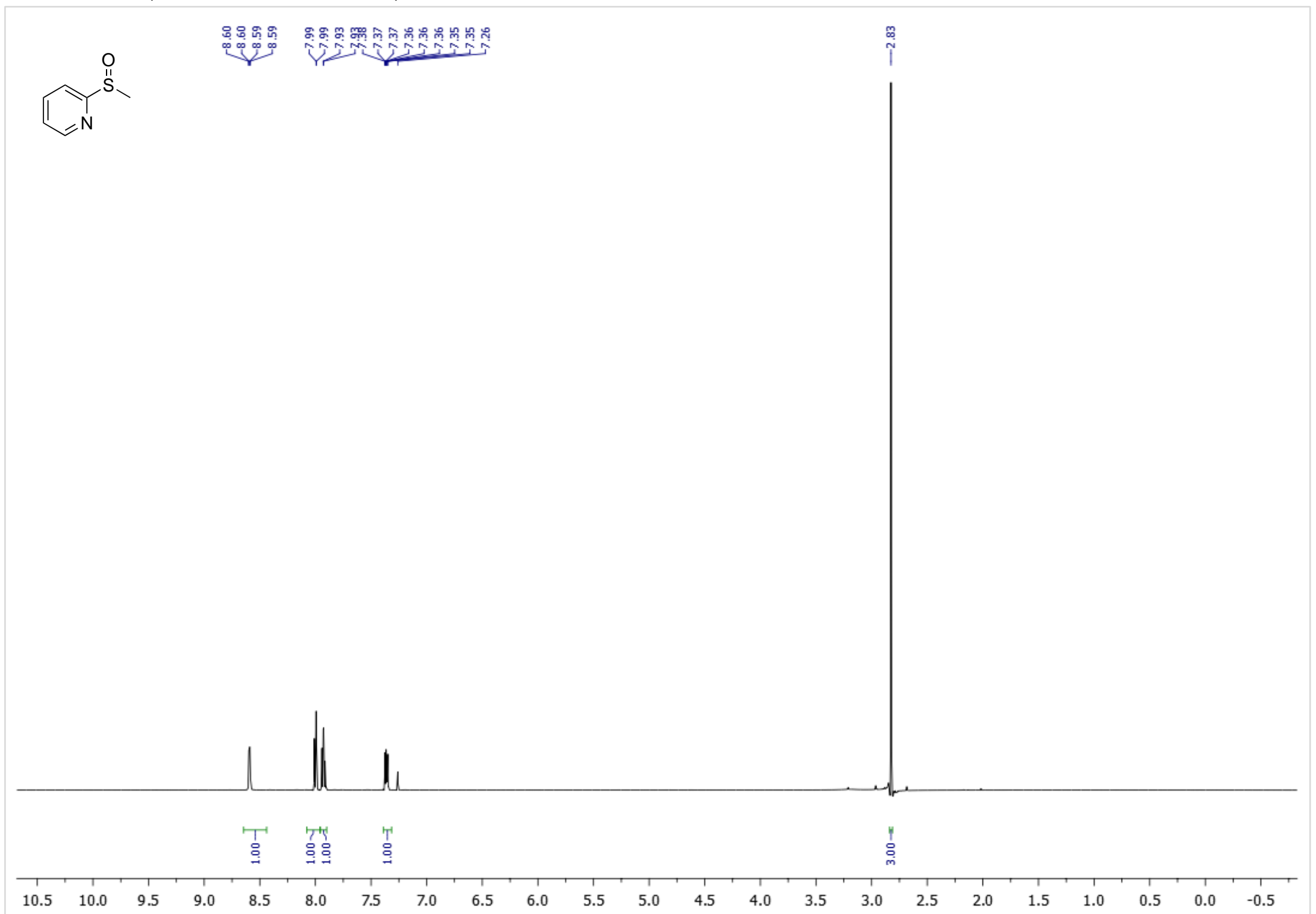


${ }^{13} \mathrm{C}\left\{{ }^{1} \mathrm{H}\right\}$ NMR $\left(126 \mathrm{MHz}, \mathrm{CDCl}_{3}, 298 \mathrm{~K}\right)$

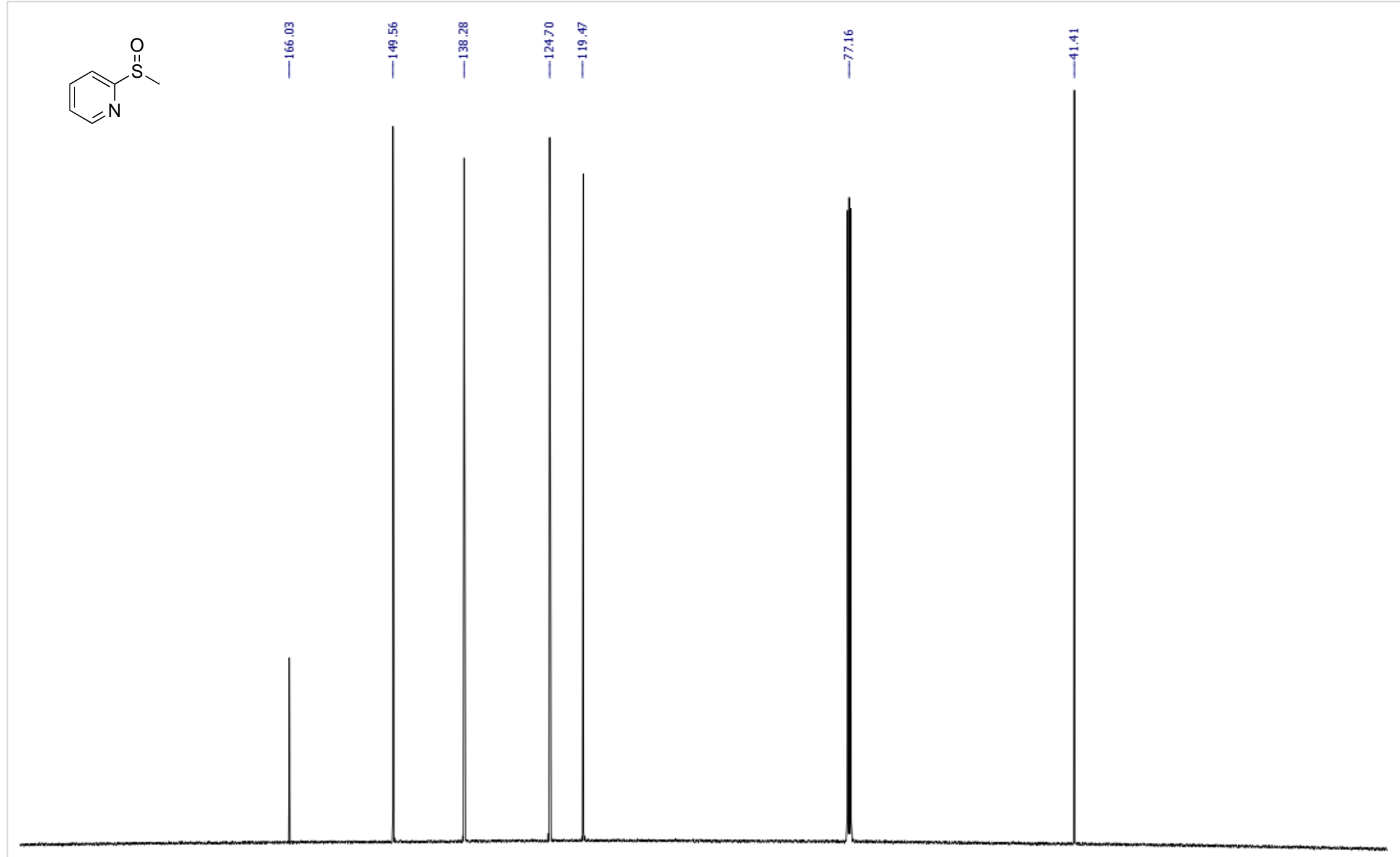

$\begin{array}{lllll}200 & 190 & 180 & 170 & 160\end{array}$

150

130

120

100

90 
${ }^{\mathbf{1}} \mathbf{H}$ NMR $\left(500 \mathrm{MHz}, \mathrm{CDCl}_{3}, 298 \mathrm{~K}\right)$

1-(butylsulfinyl)butane (2y)

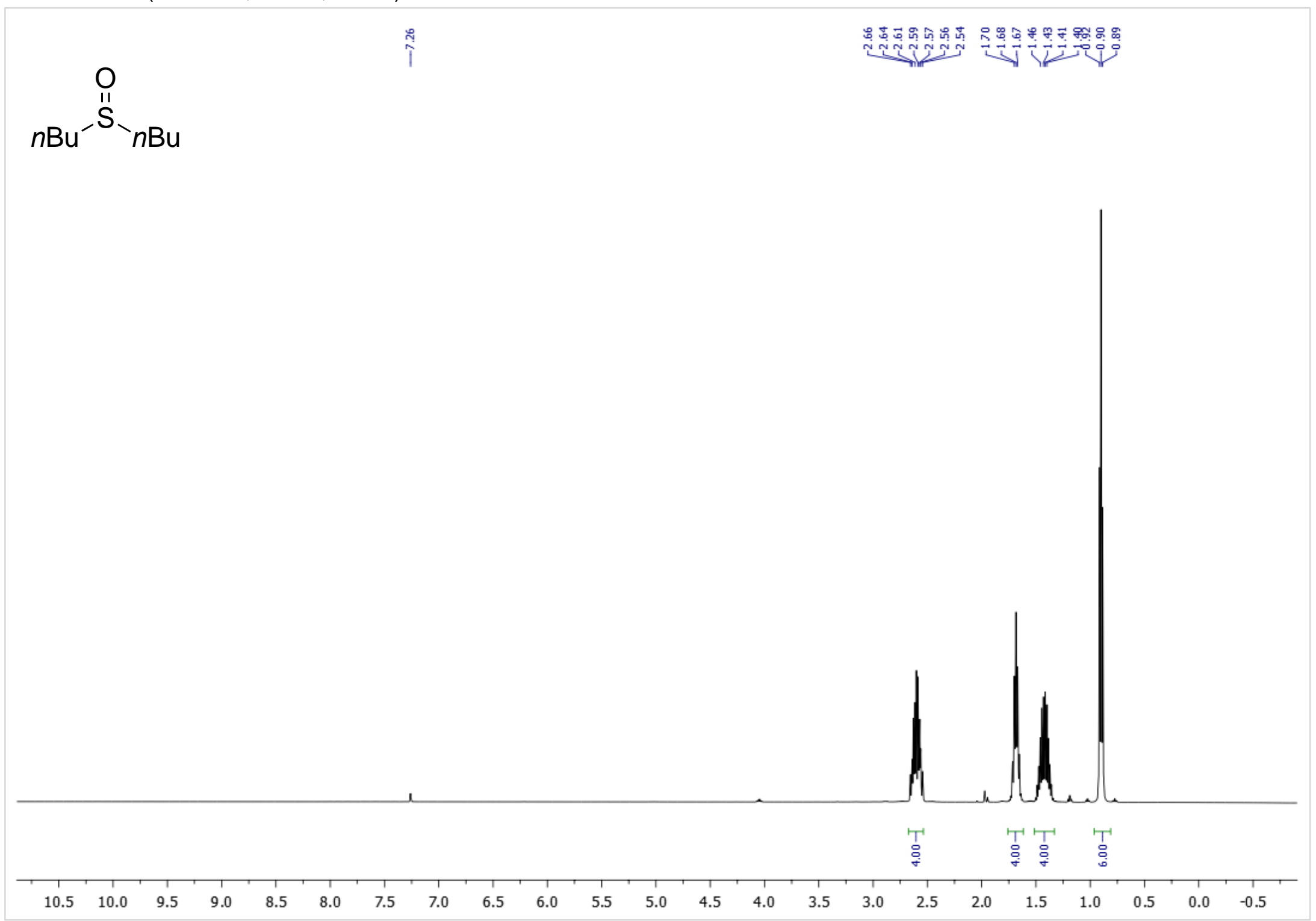


${ }^{13} \mathrm{C}\left\{{ }^{1} \mathrm{H}\right\}$ NMR $\left(101 \mathrm{MHz}, \mathrm{CDCl}_{3}, 298 \mathrm{~K}\right)$

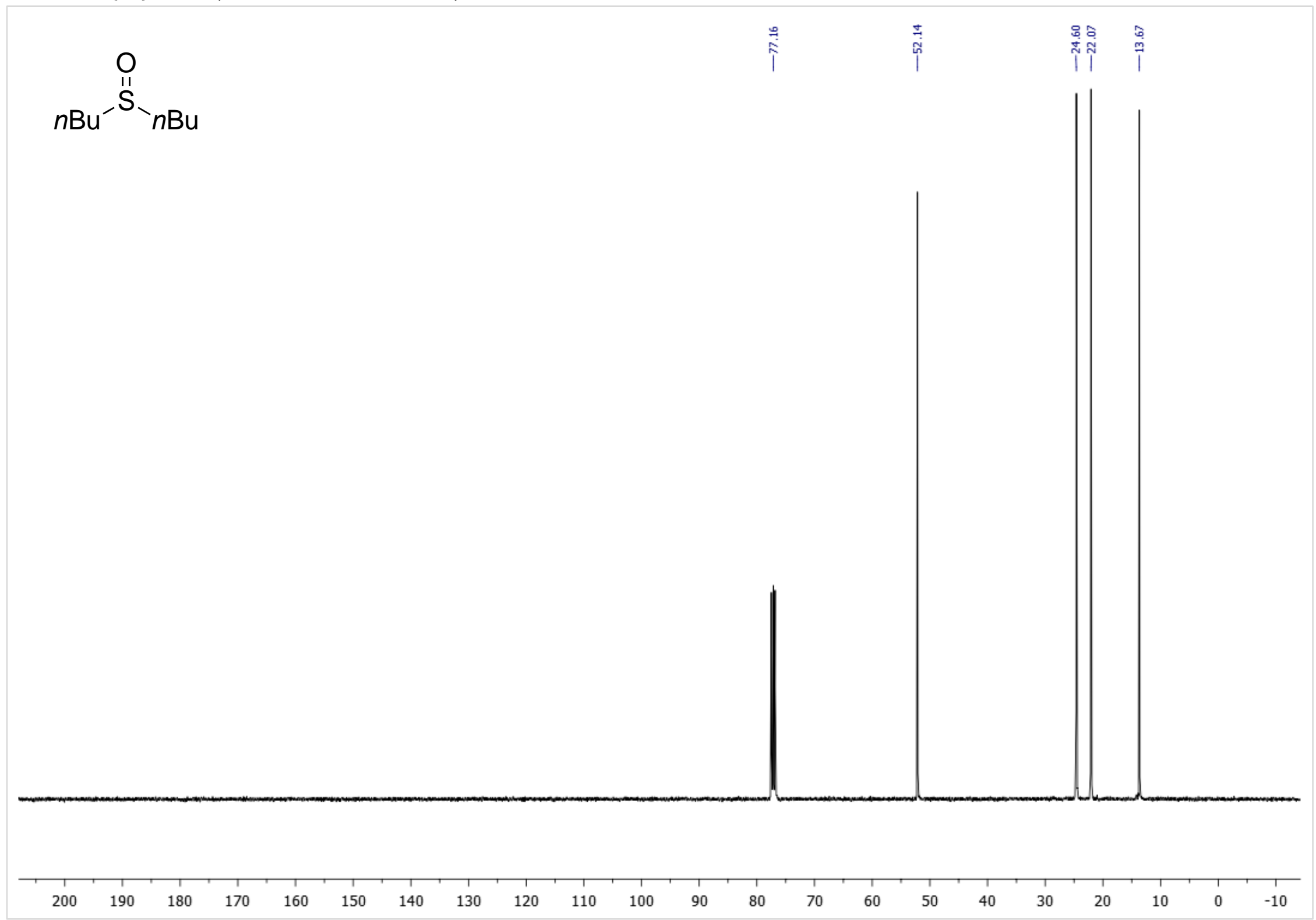


tetrahydro-2H-thiopyran 1-oxide (2z)

${ }^{1} \mathbf{H}$ NMR $\left(500 \mathrm{MHz}, \mathrm{CDCl}_{3}, 298 \mathrm{~K}\right)$

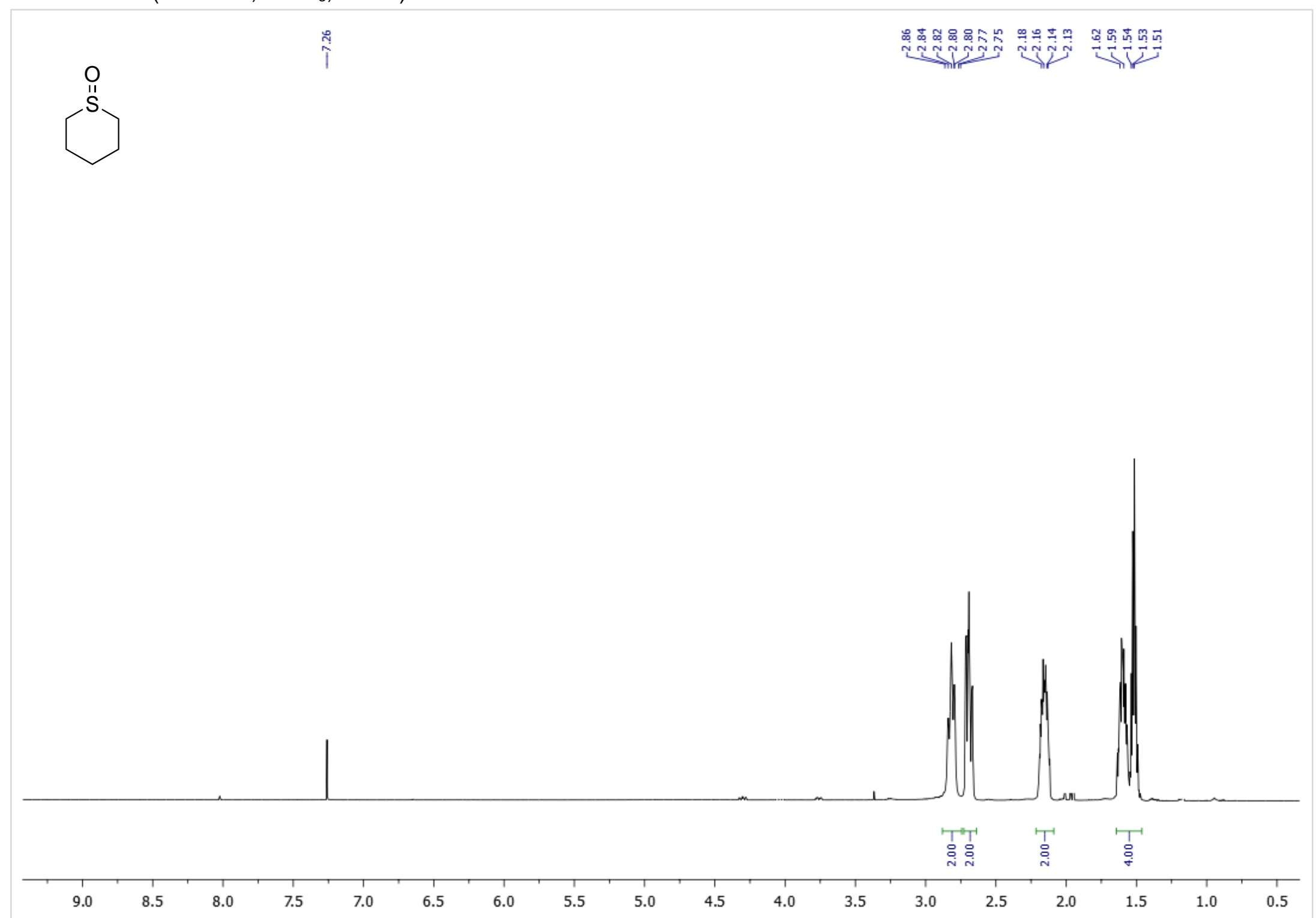


${ }^{13} \mathrm{C}\left\{{ }^{1} \mathrm{H}\right\}$ NMR $\left(126 \mathrm{MHz}, \mathrm{CDCl}_{3}, 298 \mathrm{~K}\right)$

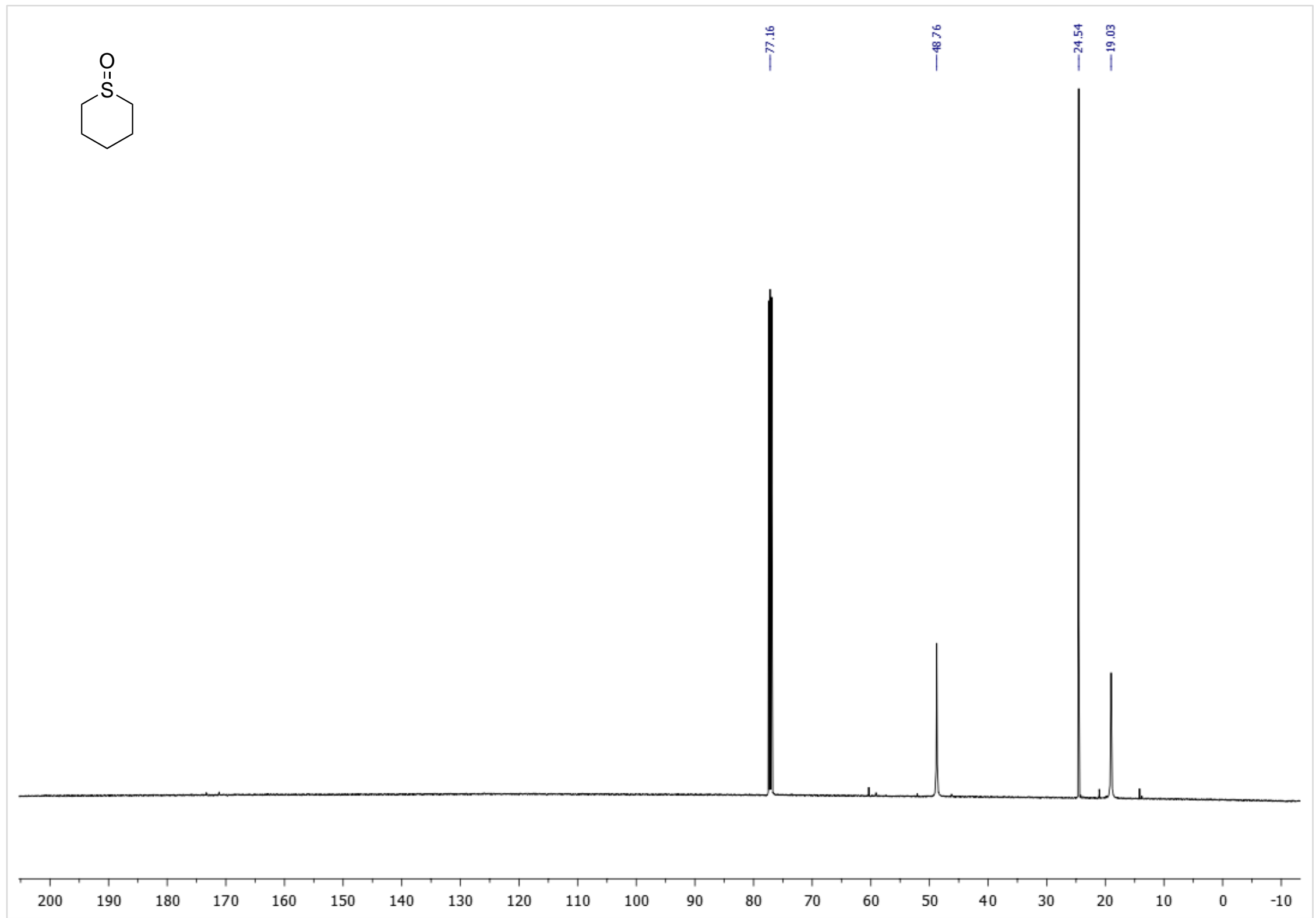


${ }^{1} \mathbf{H}$ NMR $\left(500 \mathrm{MHz}, \mathrm{CDCl}_{3}, 298 \mathrm{~K}\right)$

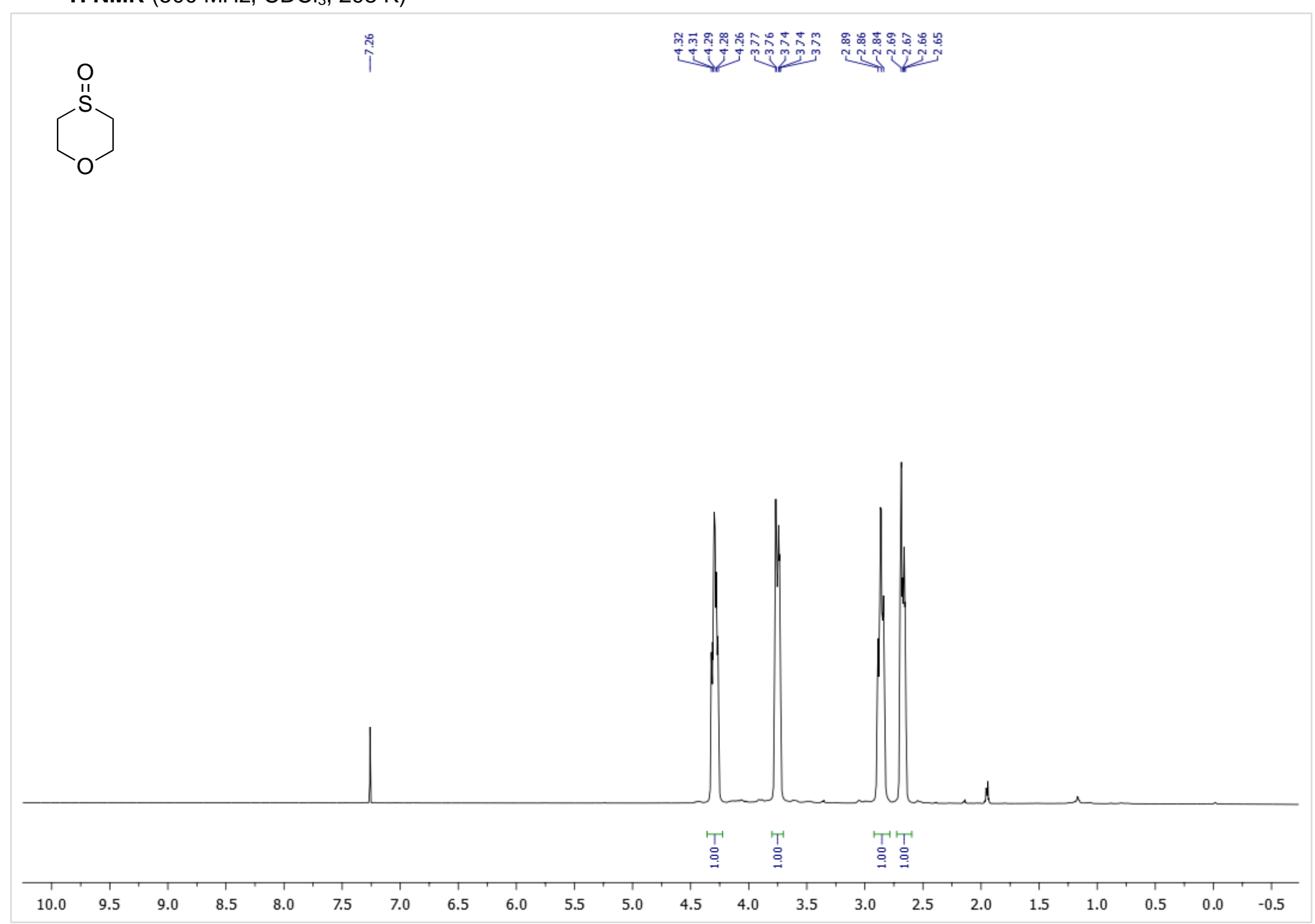


${ }^{13} \mathrm{C}\left\{{ }^{1} \mathrm{H}\right\}$ NMR $\left(126 \mathrm{MHz}, \mathrm{CDCl}_{3}, 298 \mathrm{~K}\right)$

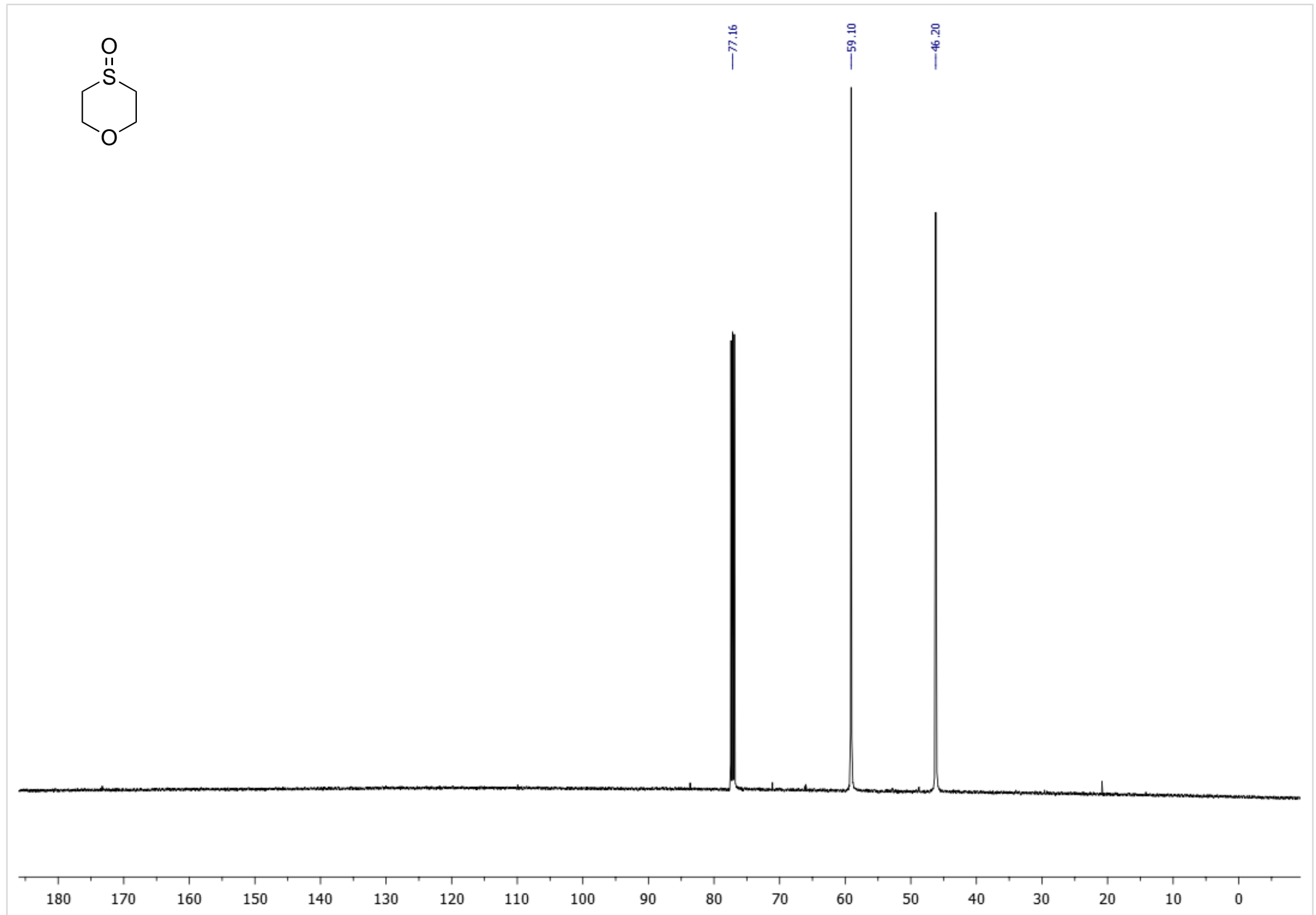


${ }^{1} \mathbf{H}$ NMR $\left(400 \mathrm{MHz}, \mathrm{CDCl}_{3}, 298 \mathrm{~K}\right)$

(methylsulfonyl)benzene (3a)

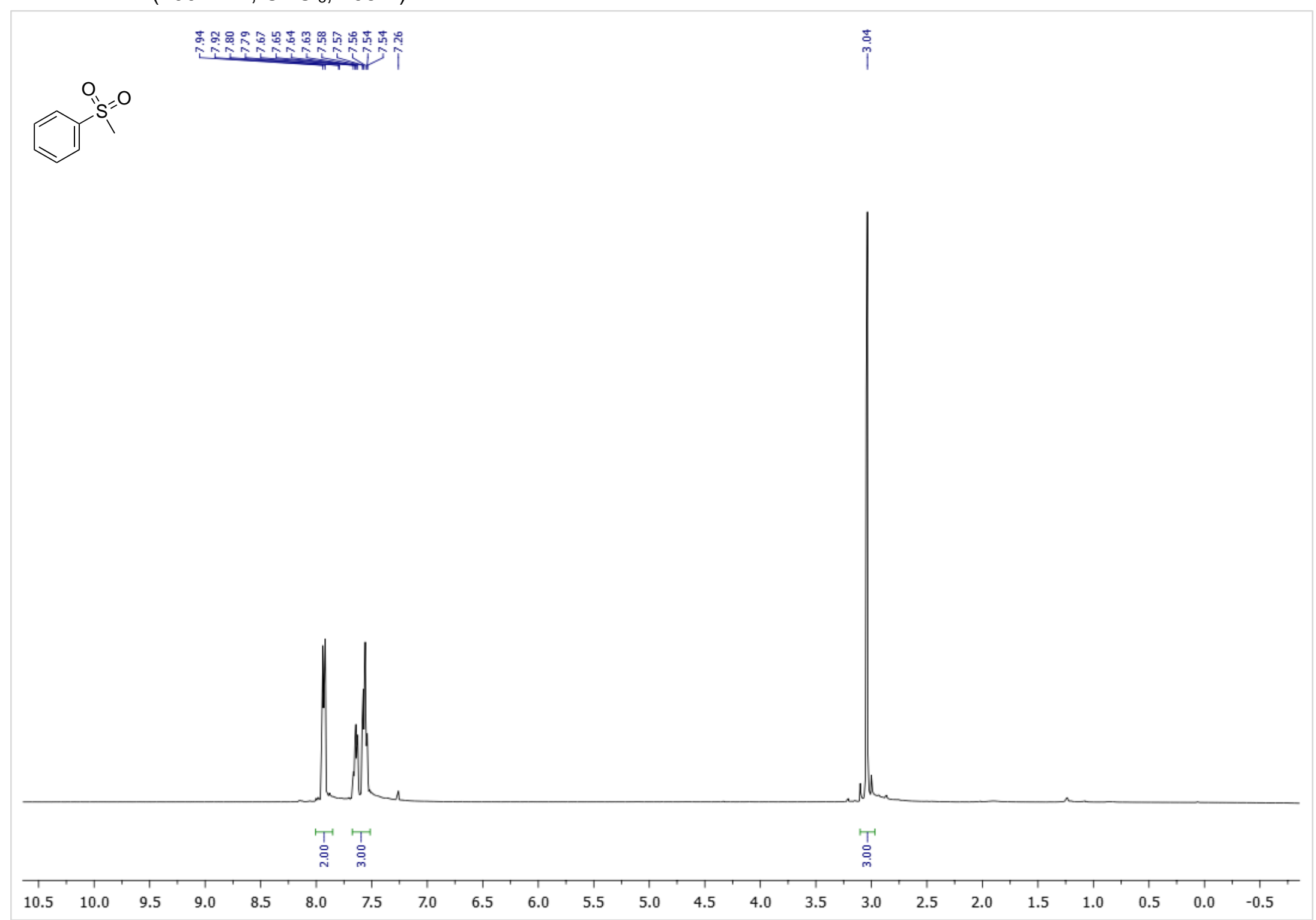


${ }^{13} \mathrm{C}\left\{{ }^{1} \mathrm{H}\right\}$ NMR $\left(101 \mathrm{MHz}, \mathrm{CDCl}_{3}, 298 \mathrm{~K}\right)$

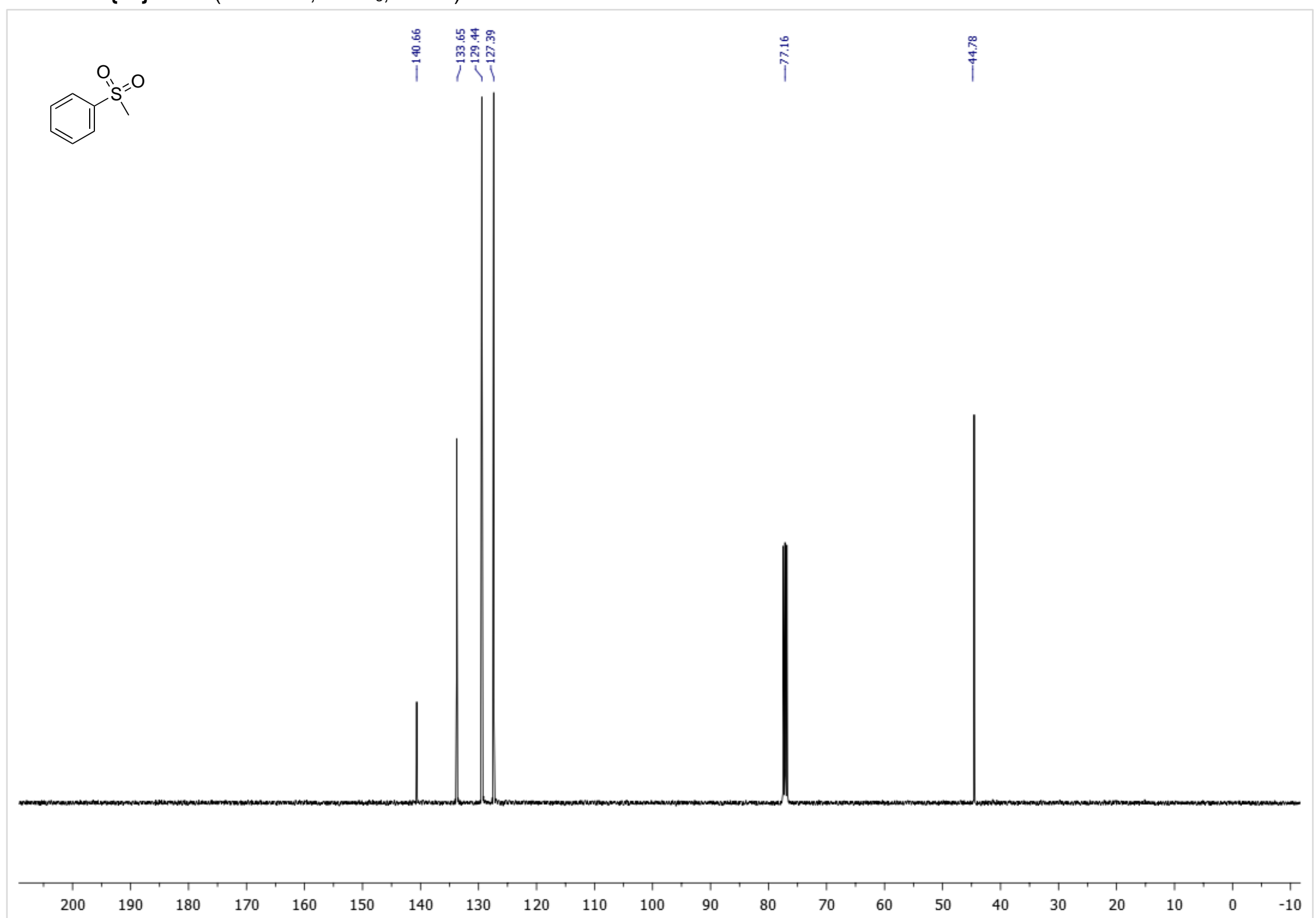


1-methyl-4-(methylsulfonyl)benzene (3b)

${ }^{1} \mathbf{H}$ NMR $\left(400 \mathrm{MHz}, \mathrm{CDCl}_{3}, 298 \mathrm{~K}\right)$

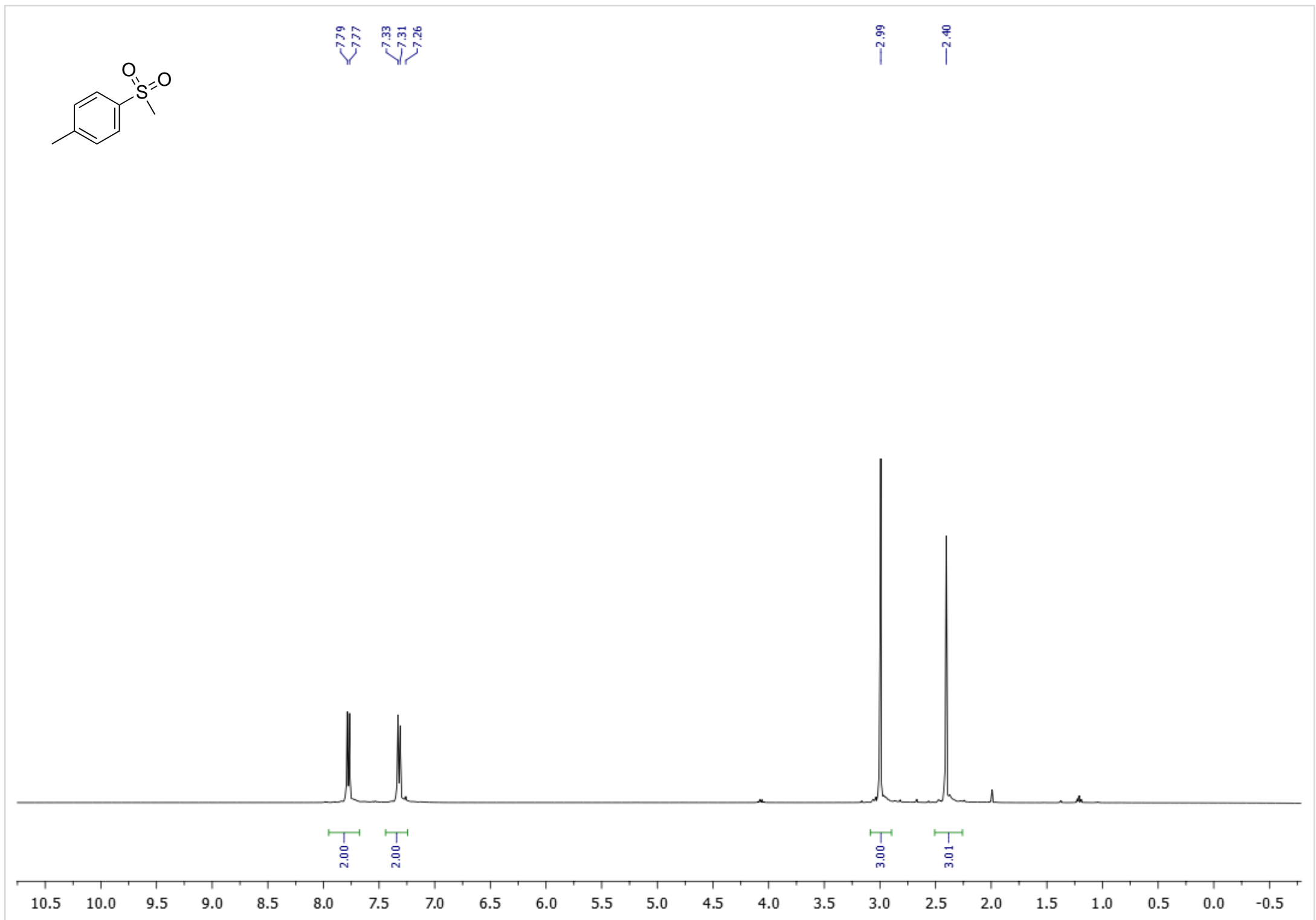


${ }^{13} \mathrm{C}\left\{{ }^{1} \mathrm{H}\right\}$ NMR $\left(101 \mathrm{MHz}, \mathrm{CDCl}_{3}, 298 \mathrm{~K}\right)$

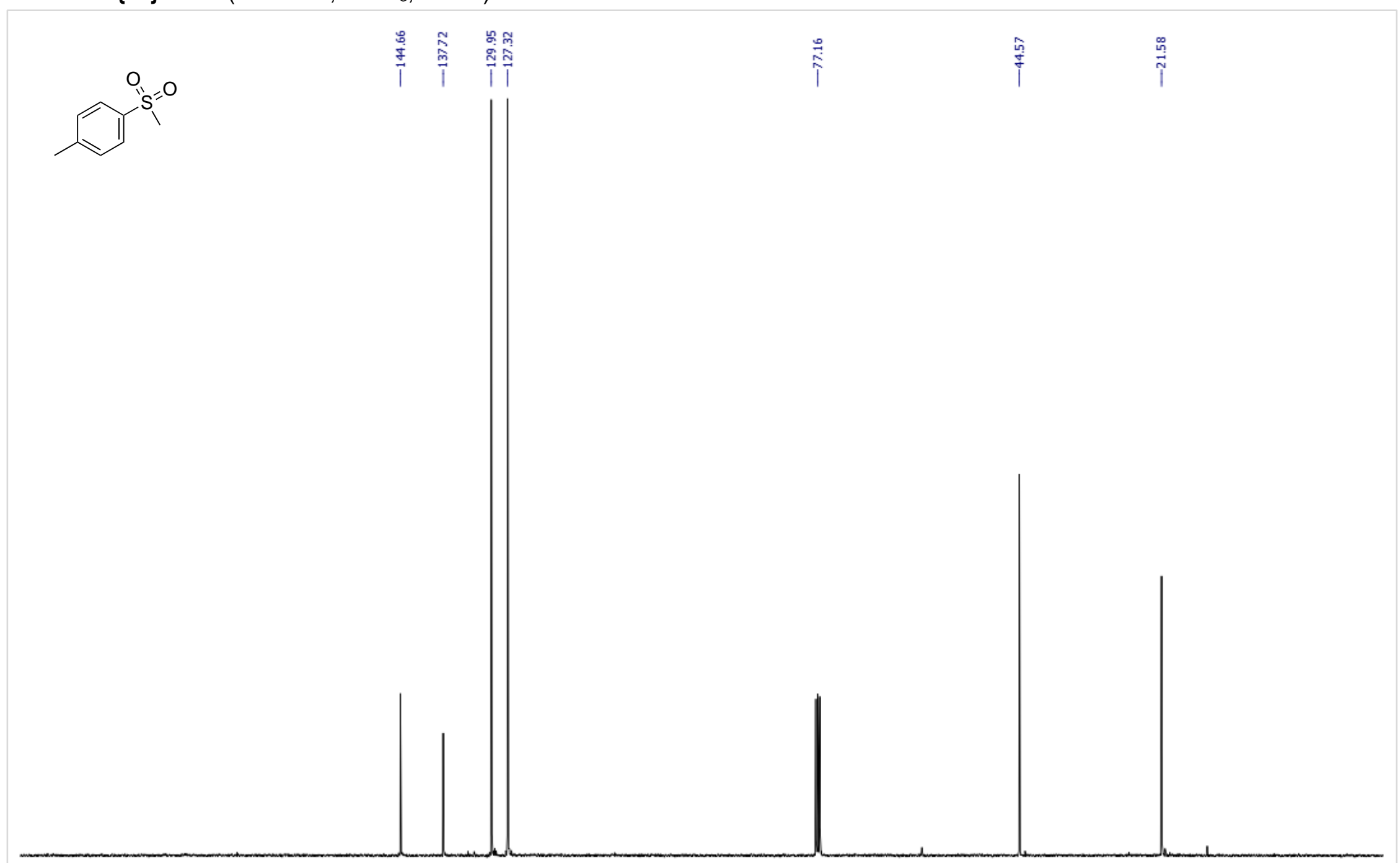


1-bromo-4-(methylsulfonyl)benzene (3c)

${ }^{1} \mathrm{H}$ NMR $\left(400 \mathrm{MHz}, \mathrm{CDCl}_{3}, 298 \mathrm{~K}\right)$

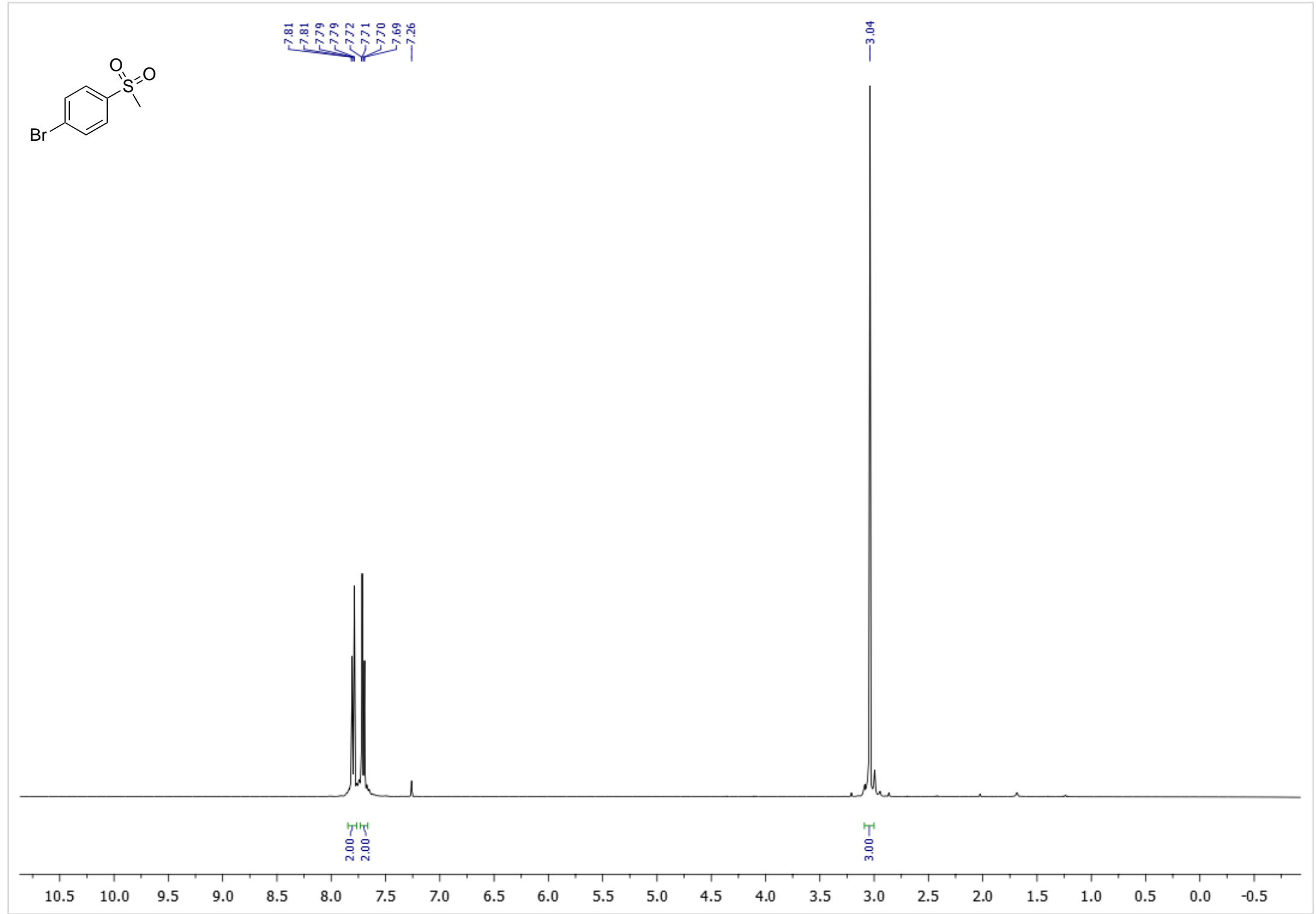


${ }^{13} \mathrm{C}\left\{{ }^{1} \mathrm{H}\right\}$ NMR $\left(101 \mathrm{MHz}, \mathrm{CDCl}_{3}, 298 \mathrm{~K}\right)$

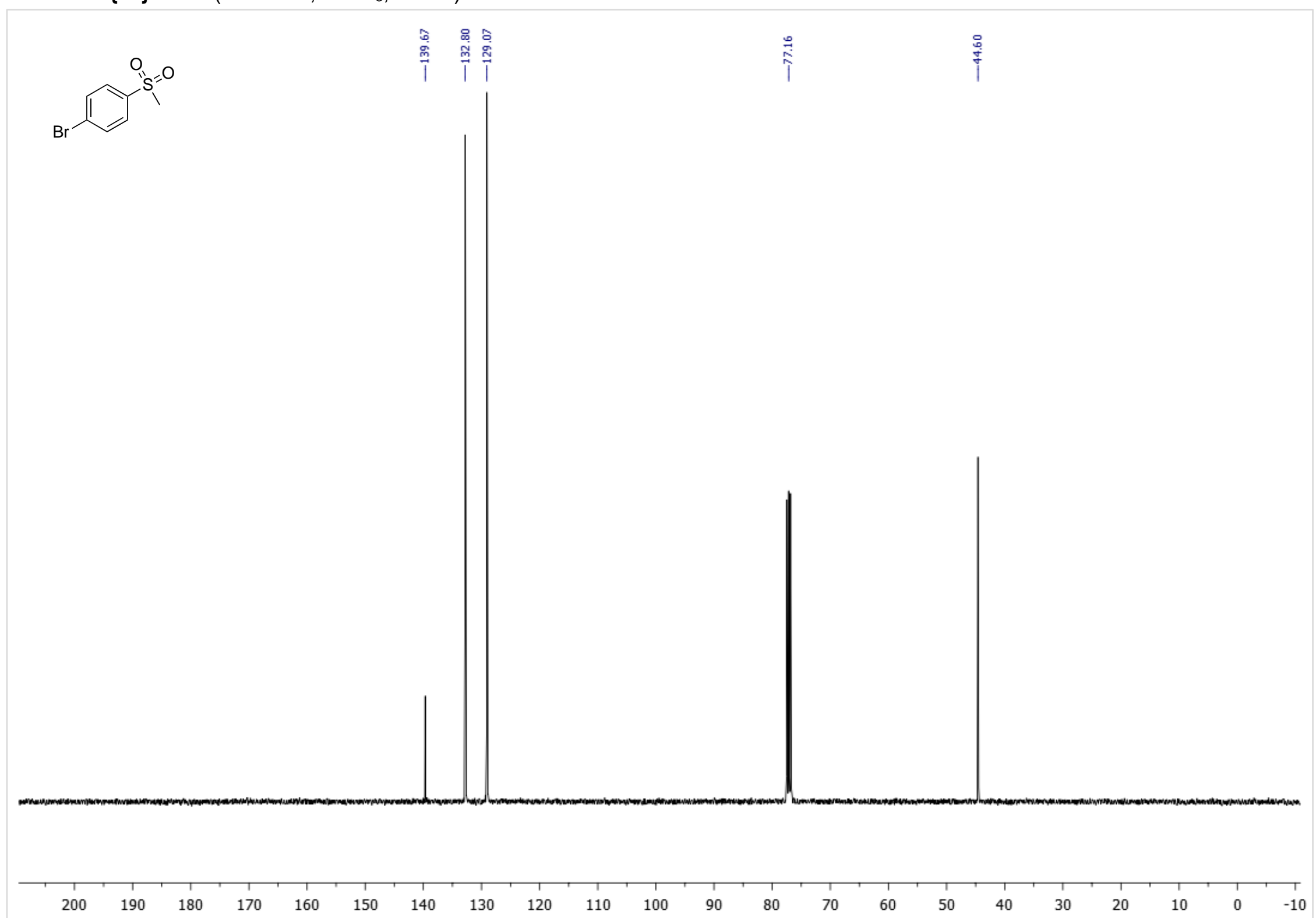


1-chloro-4-(methylsulfonyl)benzene (3d)

${ }^{1} \mathbf{H}$ NMR $\left(400 \mathrm{MHz}, \mathrm{CDCl}_{3}, 298 \mathrm{~K}\right)$

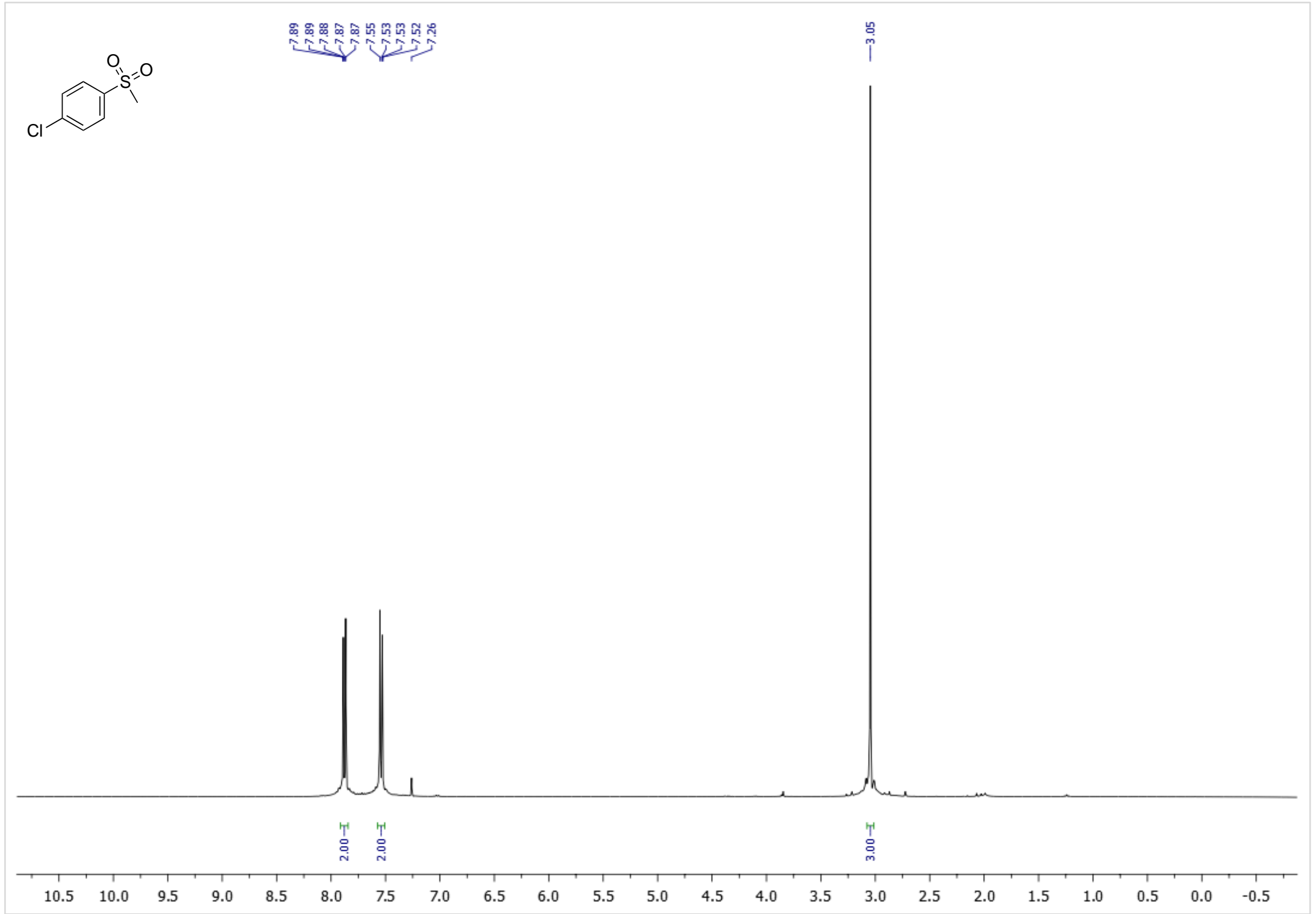


${ }^{13} \mathrm{C}\left\{{ }^{1} \mathrm{H}\right\}$ NMR $\left(101 \mathrm{MHz}, \mathrm{CDCl}_{3}, 298 \mathrm{~K}\right)$

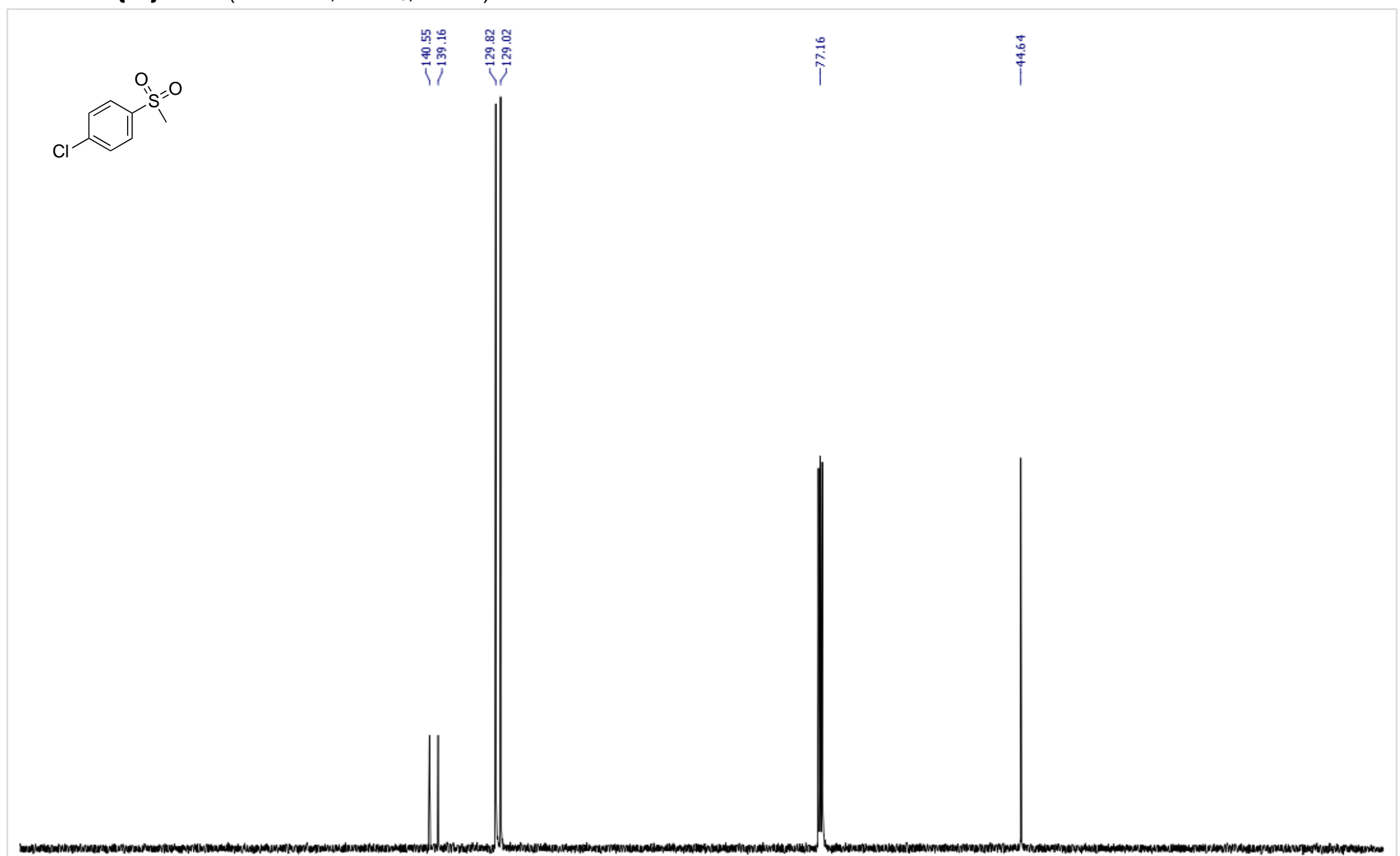

$200 \quad 190$

170

150

130

120

$110 \quad 100$

90

80

70

60

50

40 
1-fluoro-4-(methylsulfonyl)benzene (3e)

${ }^{1} \mathrm{H}$ NMR $\left(500 \mathrm{MHz}, \mathrm{CDCl}_{3}, 298 \mathrm{~K}\right)$

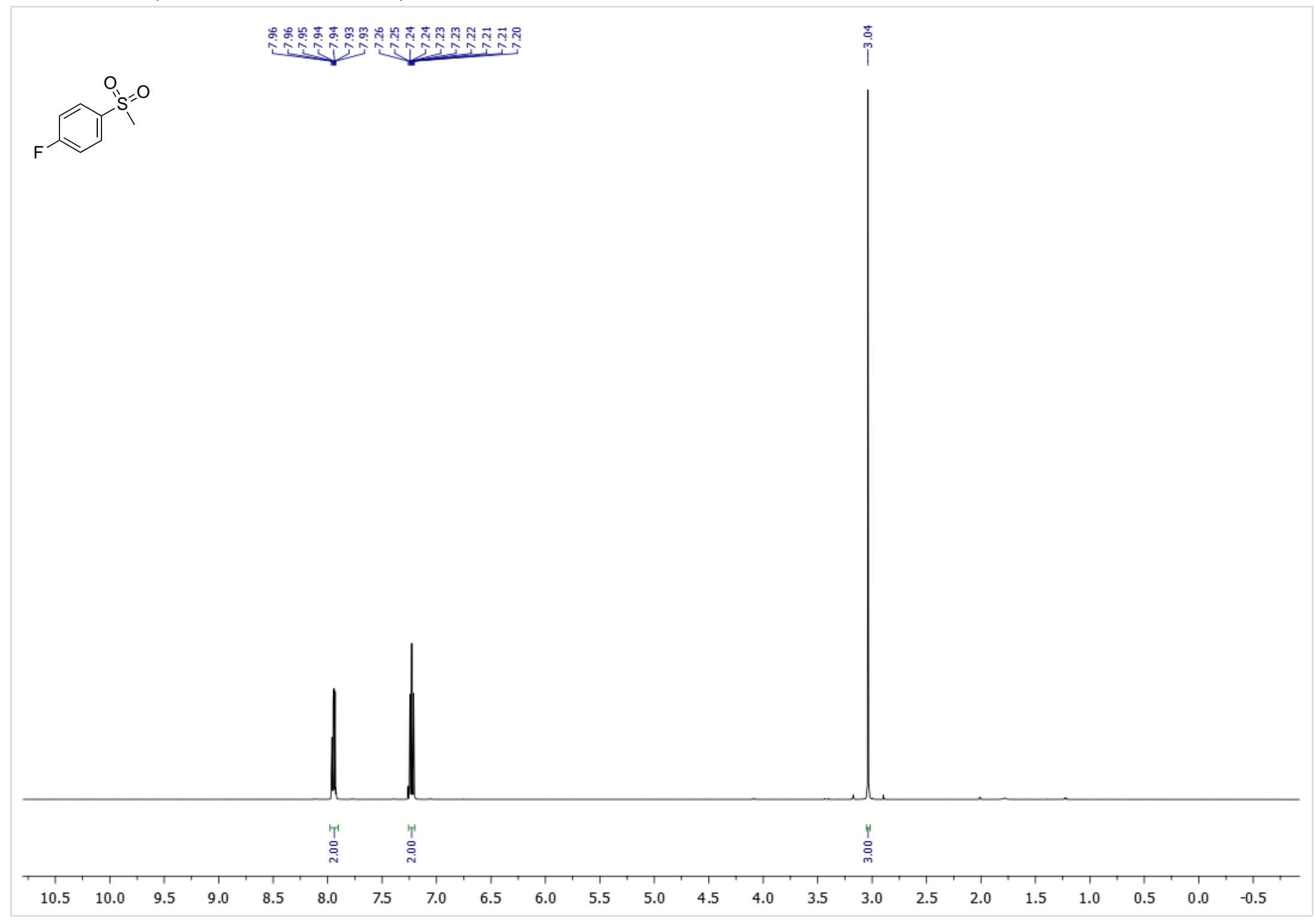


${ }^{13} \mathrm{C}\left\{{ }^{1} \mathrm{H}\right\}$ NMR $\left(101 \mathrm{MHz}, \mathrm{CDCl}_{3}, 298 \mathrm{~K}\right)$

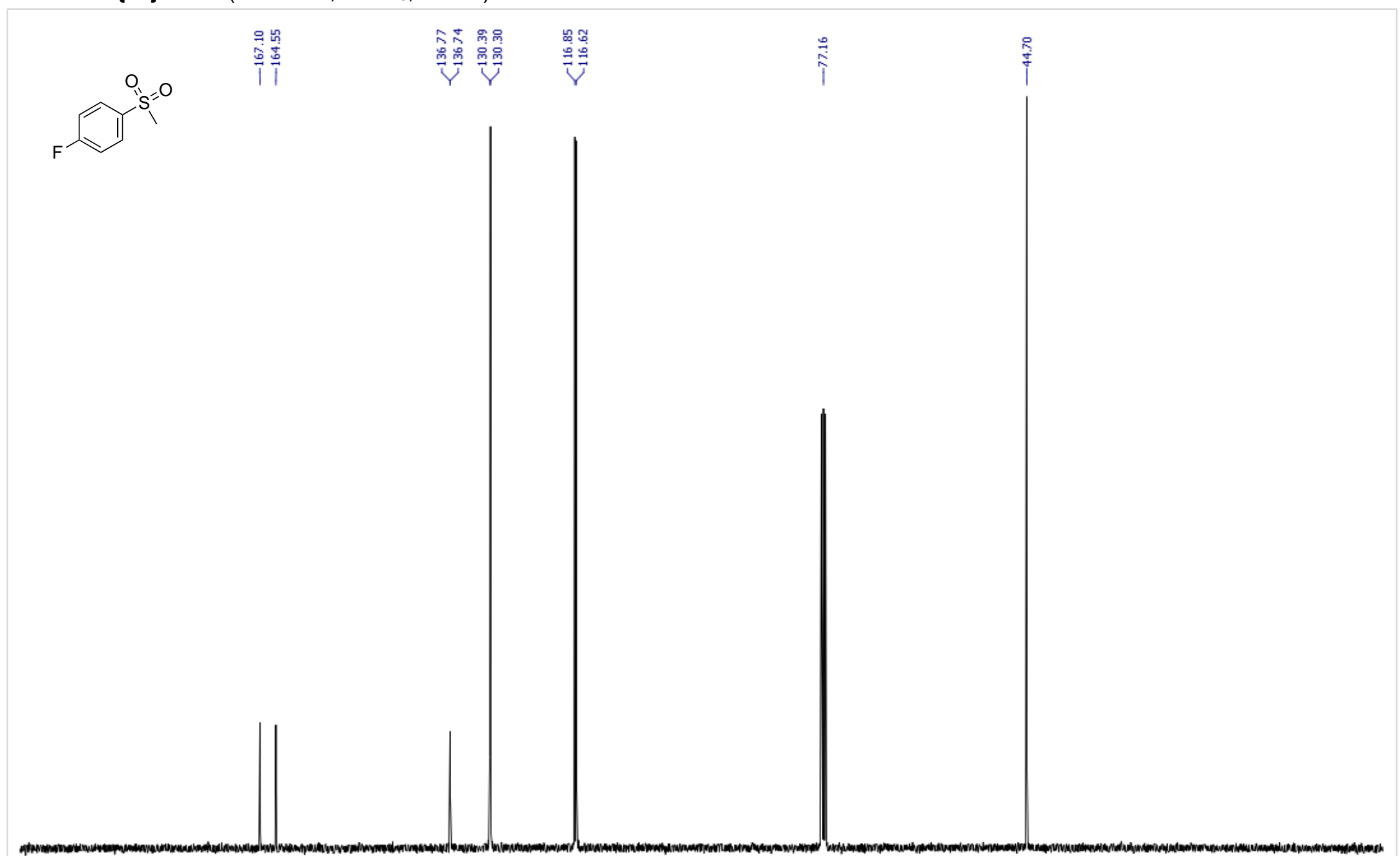


${ }^{19} \mathrm{~F}\left\{{ }^{1} \mathrm{H}\right\} \operatorname{NMR}\left(471 \mathrm{MHz}, \mathrm{CDCl}_{3}, 298 \mathrm{~K}\right)$

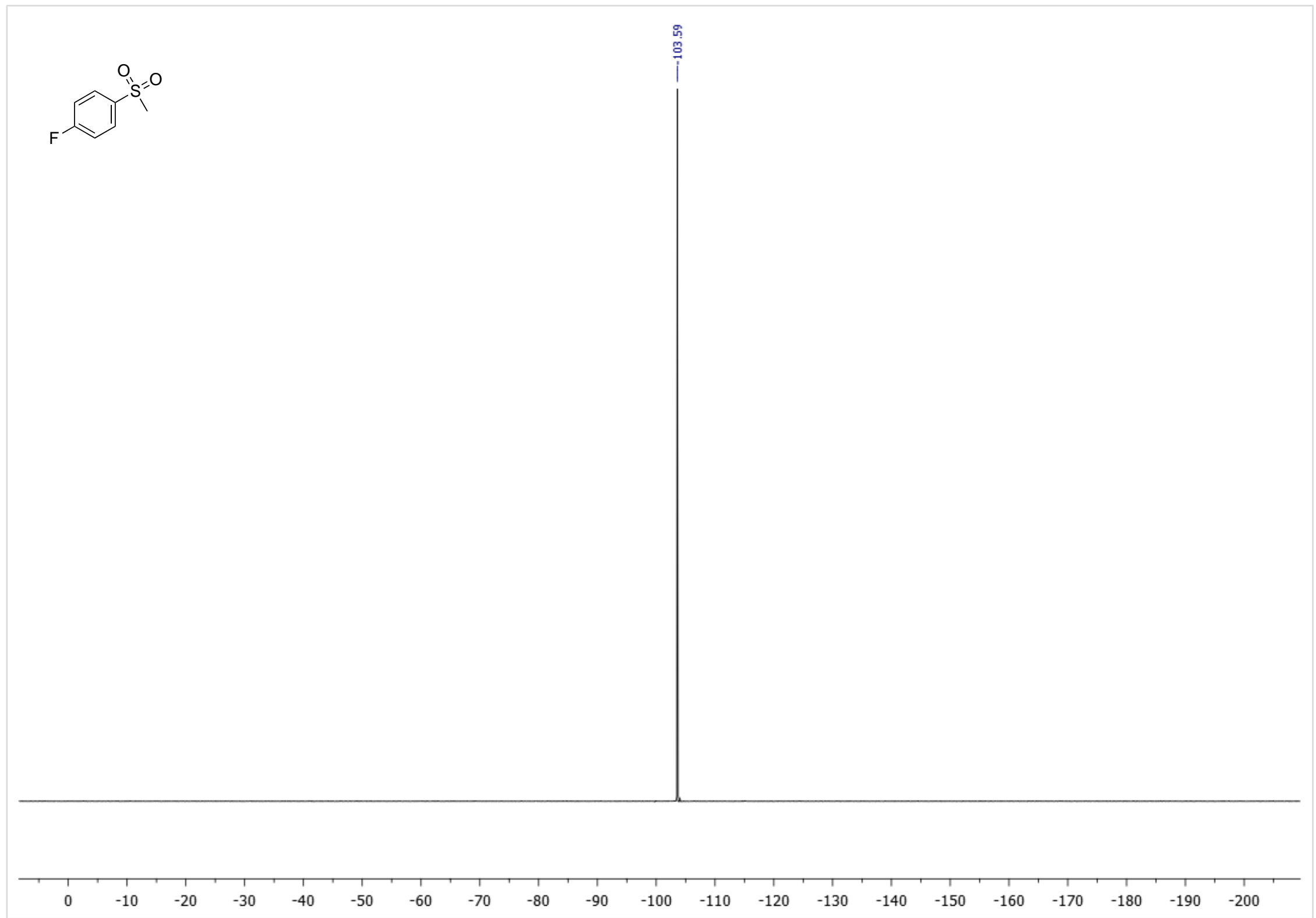


4-(methylsulfonyl)benzonitrile (3f)

${ }^{1} \mathrm{H}$ NMR $\left(500 \mathrm{MHz}, \mathrm{CDCl}_{3}, 298 \mathrm{~K}\right)$

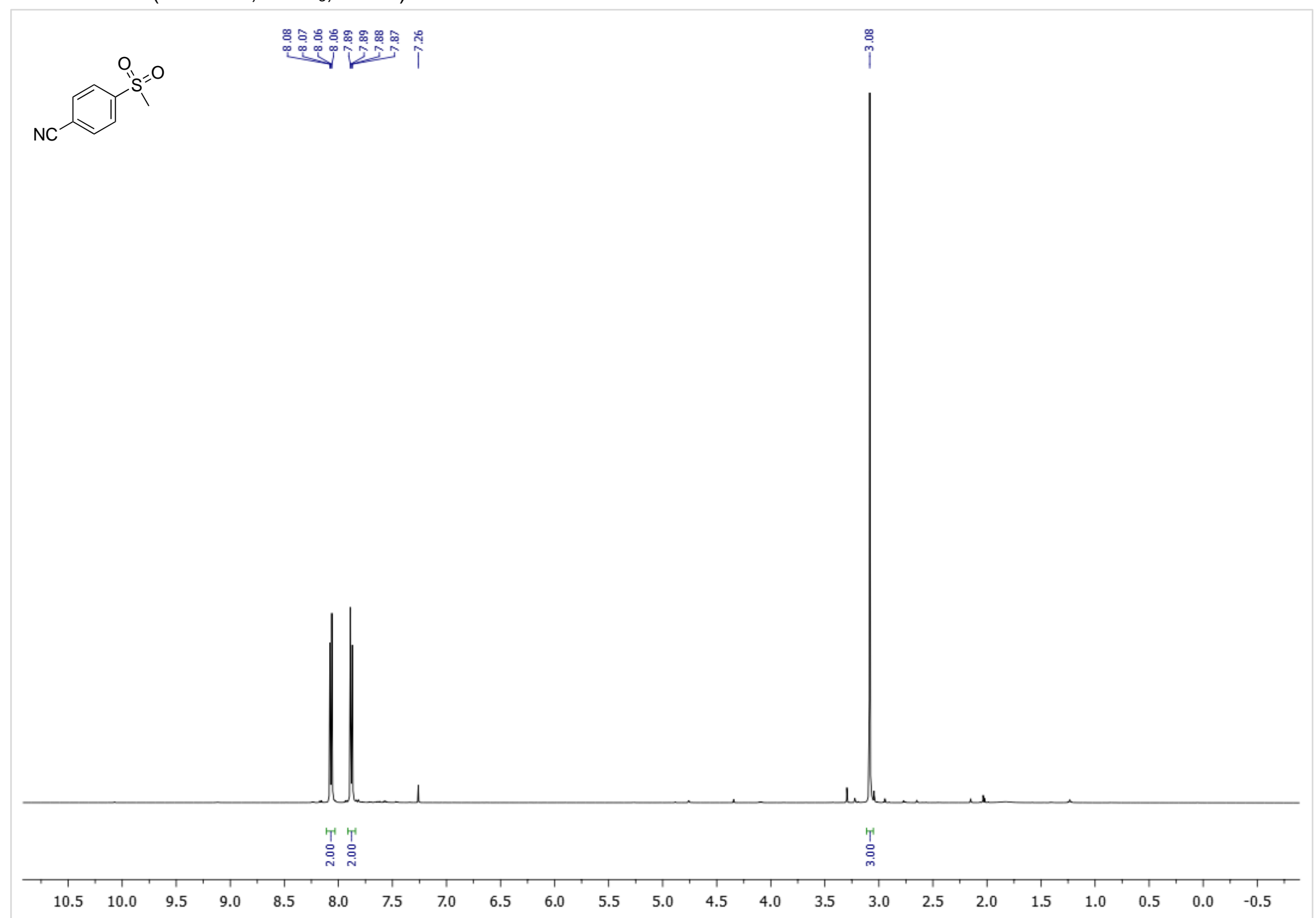


${ }^{13} \mathrm{C}\left\{{ }^{1} \mathrm{H}\right\}$ NMR $\left(101 \mathrm{MHz}, \mathrm{CDCl}_{3}, 298 \mathrm{~K}\right)$

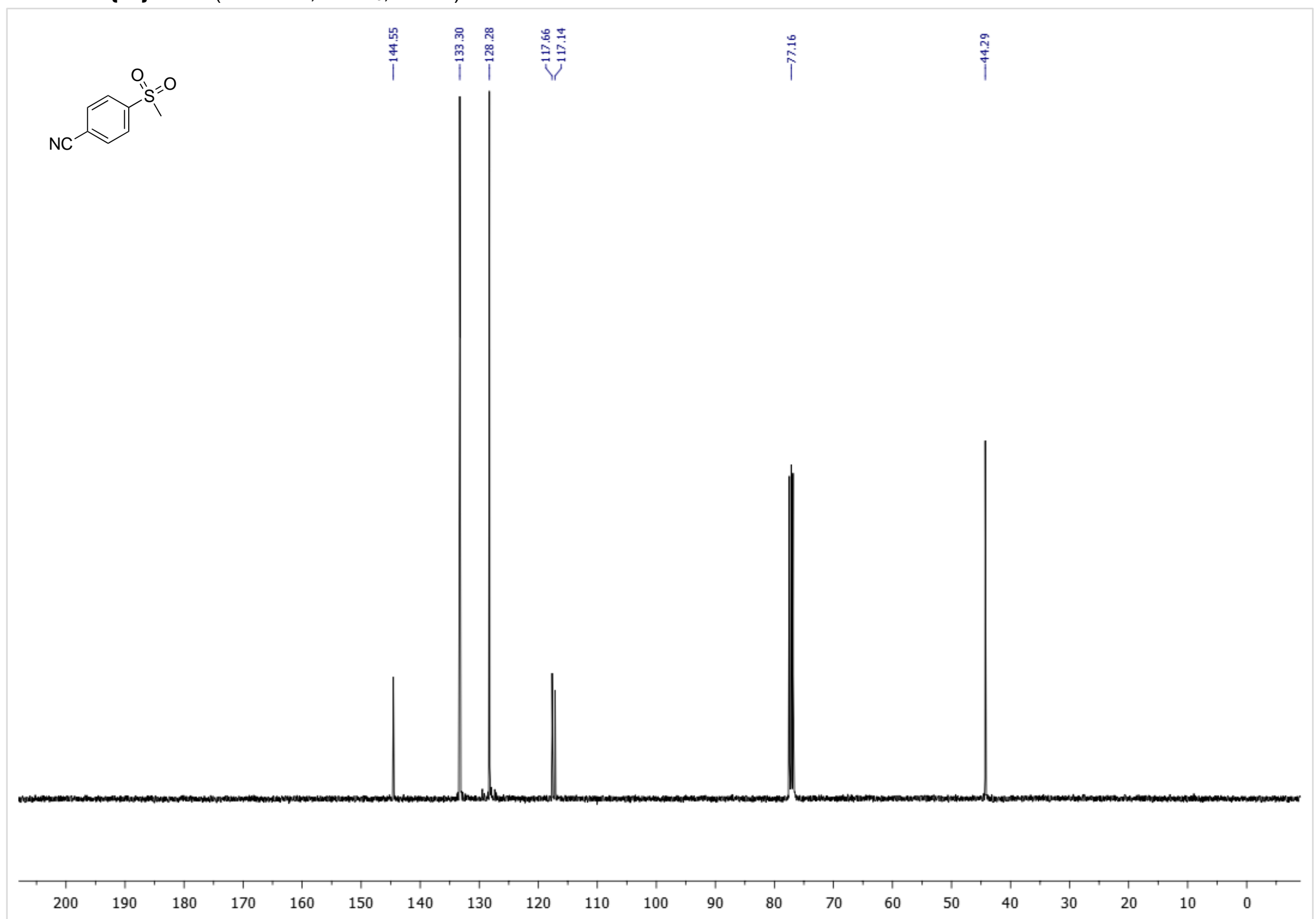


1-methoxy-4-(methylsulfonyl)benzene (3g)

${ }^{1} \mathrm{H}$ NMR $\left(400 \mathrm{MHz}, \mathrm{CDCl}_{3}, 298 \mathrm{~K}\right)$

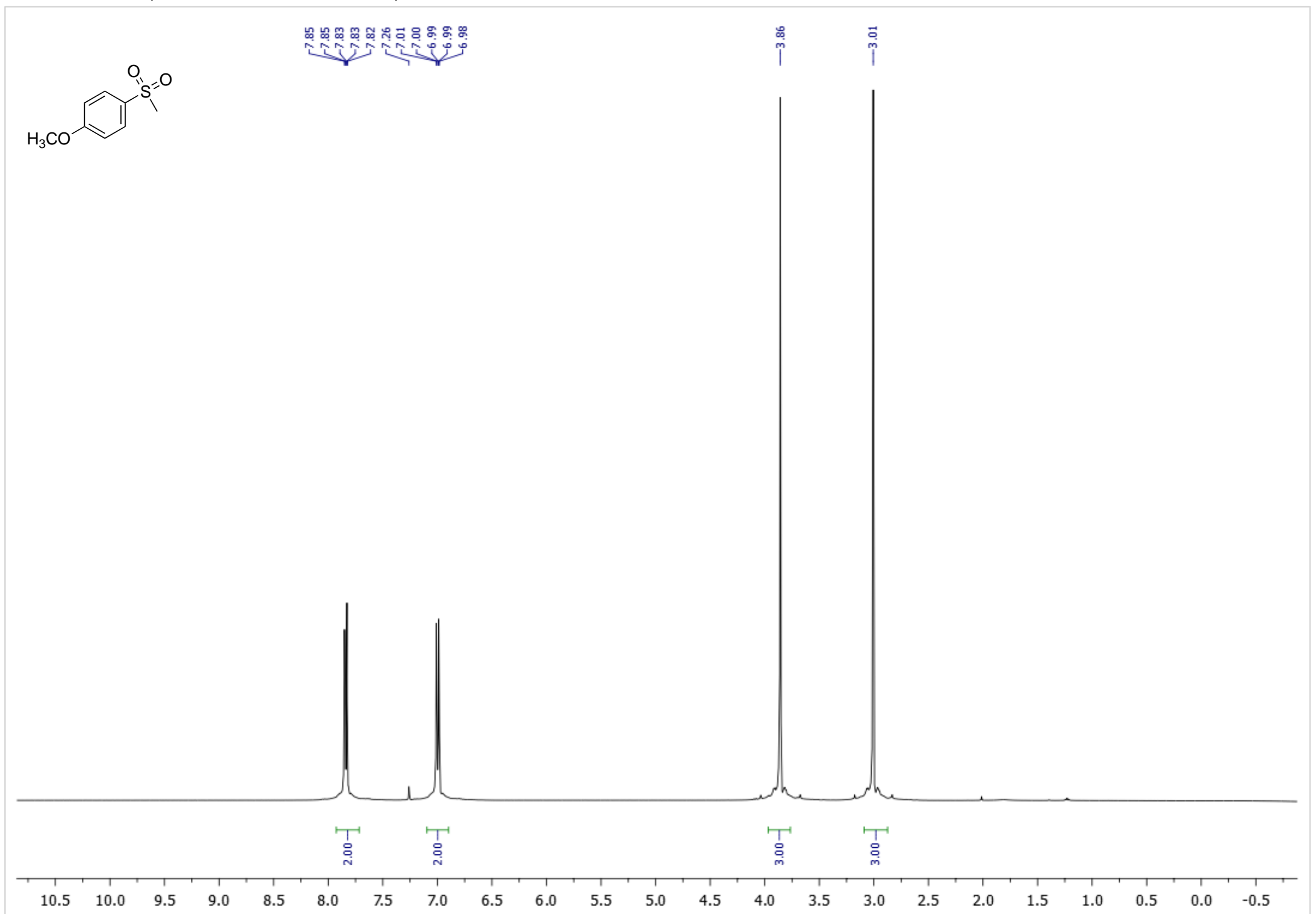


${ }^{13} \mathrm{C}\left\{{ }^{1} \mathrm{H}\right\}$ NMR $\left(101 \mathrm{MHz}, \mathrm{CDCl}_{3}, 298 \mathrm{~K}\right)$

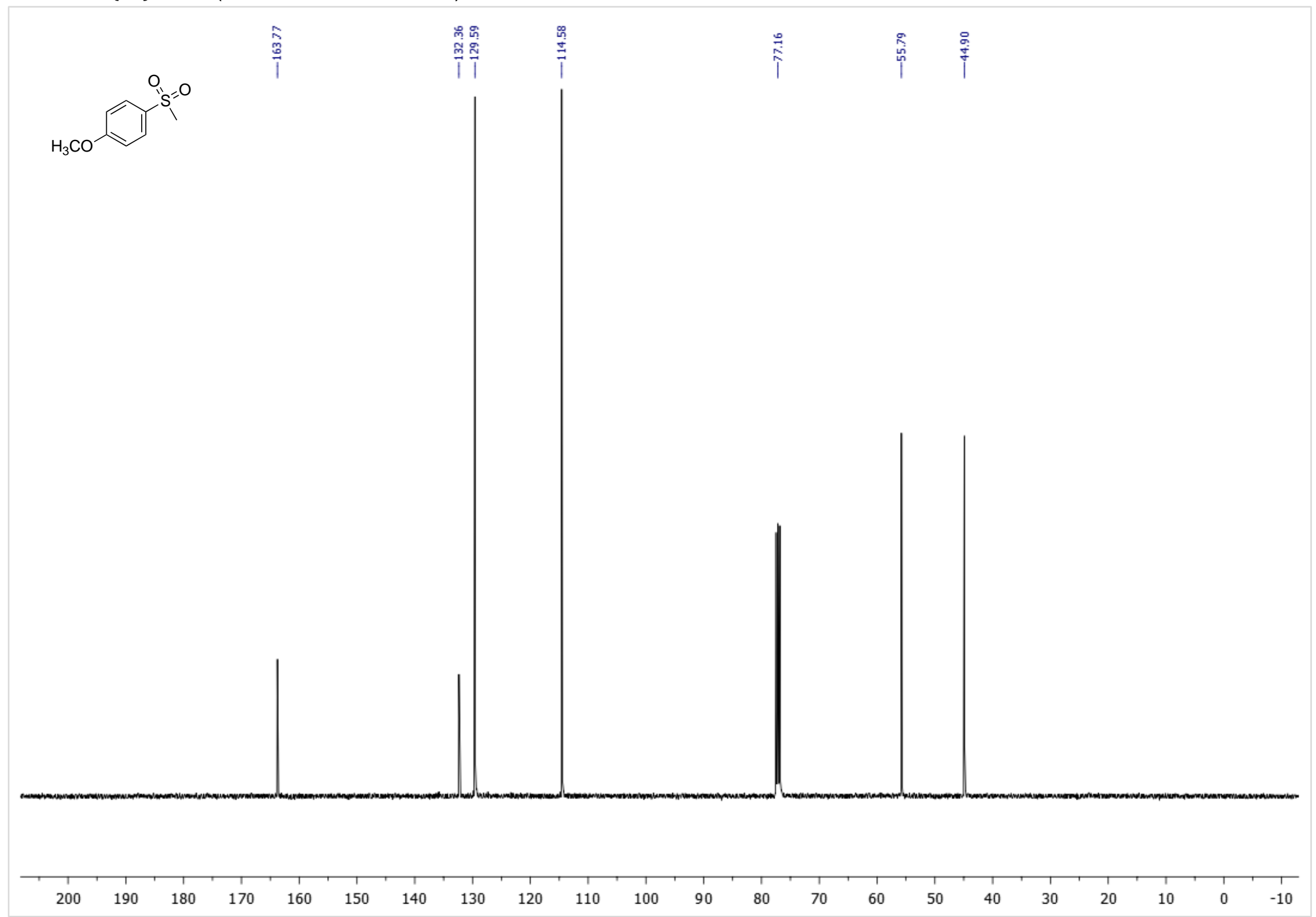


4-(methylsulfonyl)benzaldehyde (3h)

${ }^{1} \mathbf{H}$ NMR $\left(400 \mathrm{MHz}, \mathrm{CDCl}_{3}, 298 \mathrm{~K}\right)$

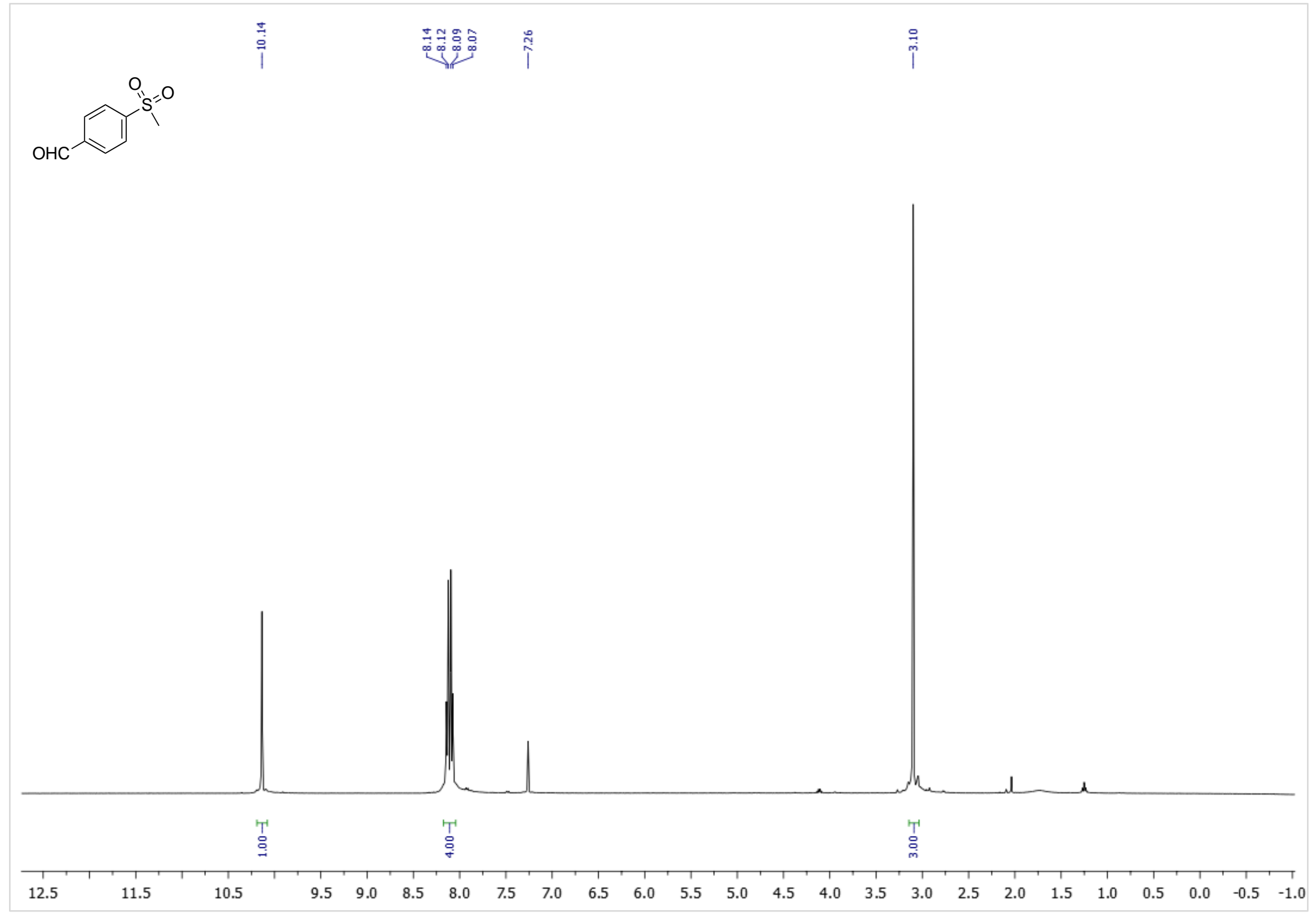

S81 
${ }^{13} \mathrm{C}\left\{{ }^{1} \mathrm{H}\right\}$ NMR $\left(101 \mathrm{MHz}, \mathrm{CDCl}_{3}, 298 \mathrm{~K}\right)$

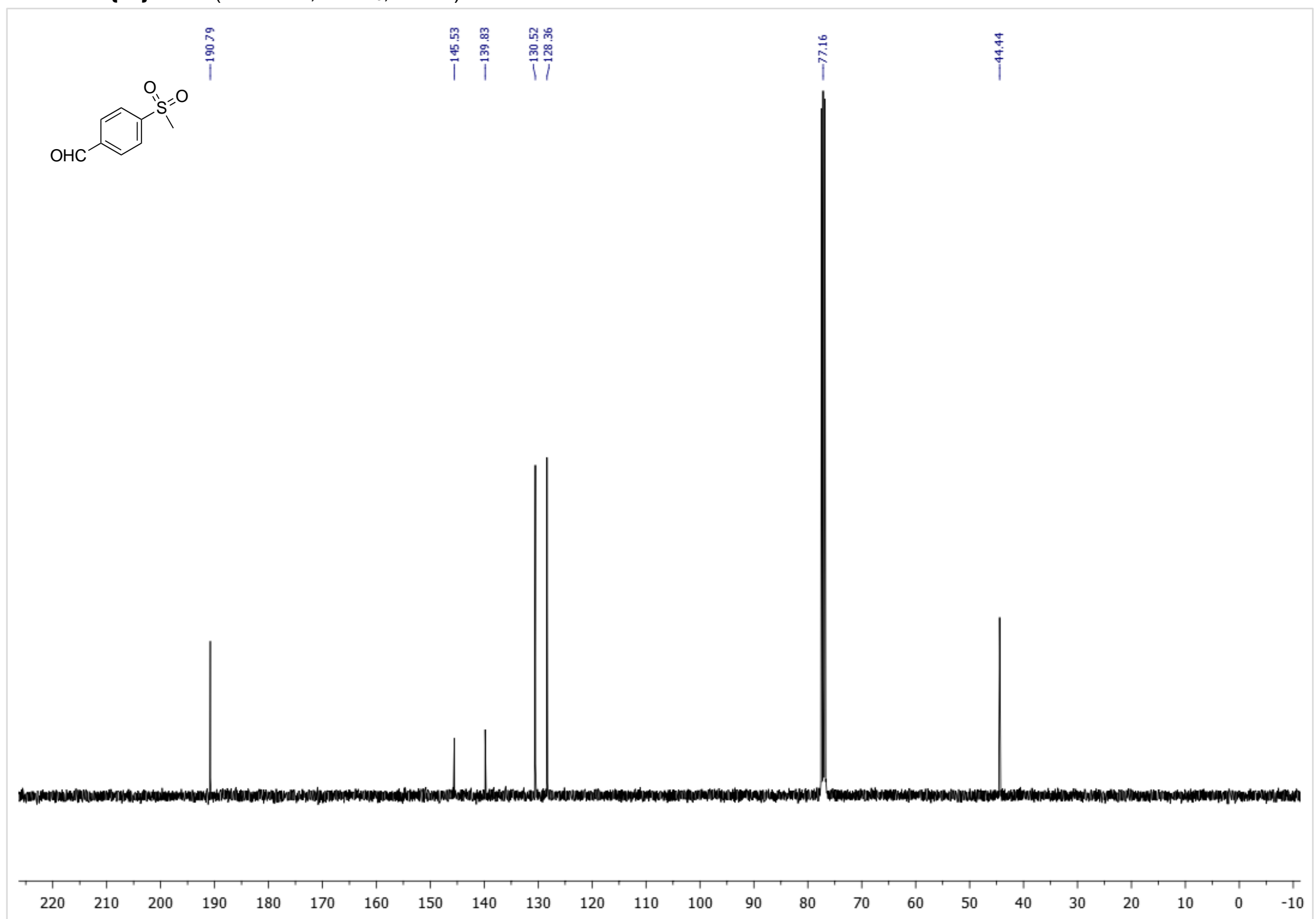


1-(methylsulfonyl)-4-nitrobenzene (3i)

${ }^{1} \mathbf{H}$ NMR $\left(500 \mathrm{MHz}, \mathrm{CDCl}_{3}, 298 \mathrm{~K}\right)$

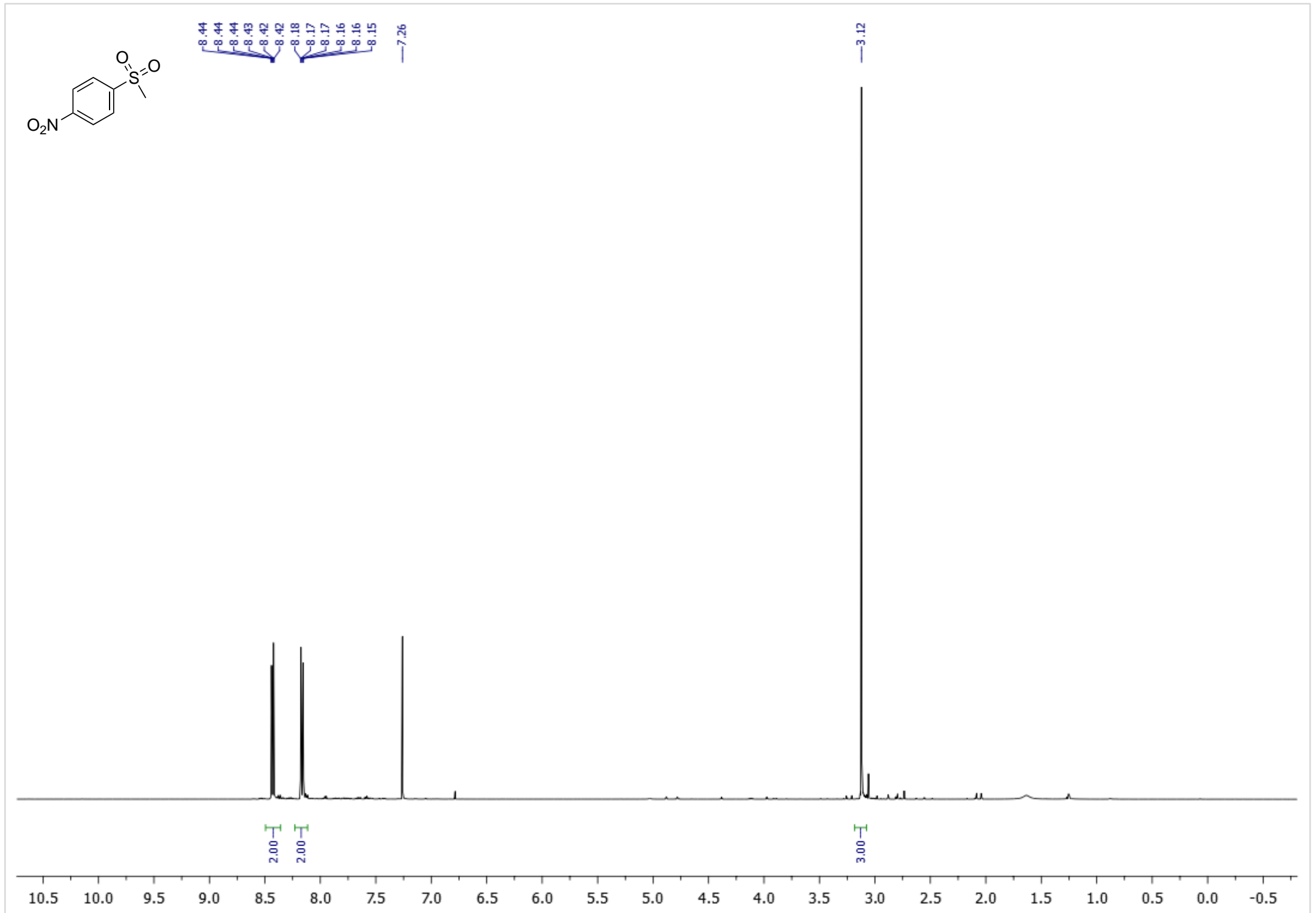


${ }^{13} \mathrm{C}\left\{{ }^{1} \mathrm{H}\right\}$ NMR $\left(126 \mathrm{MHz}, \mathrm{CDCl}_{3}, 298 \mathrm{~K}\right)$

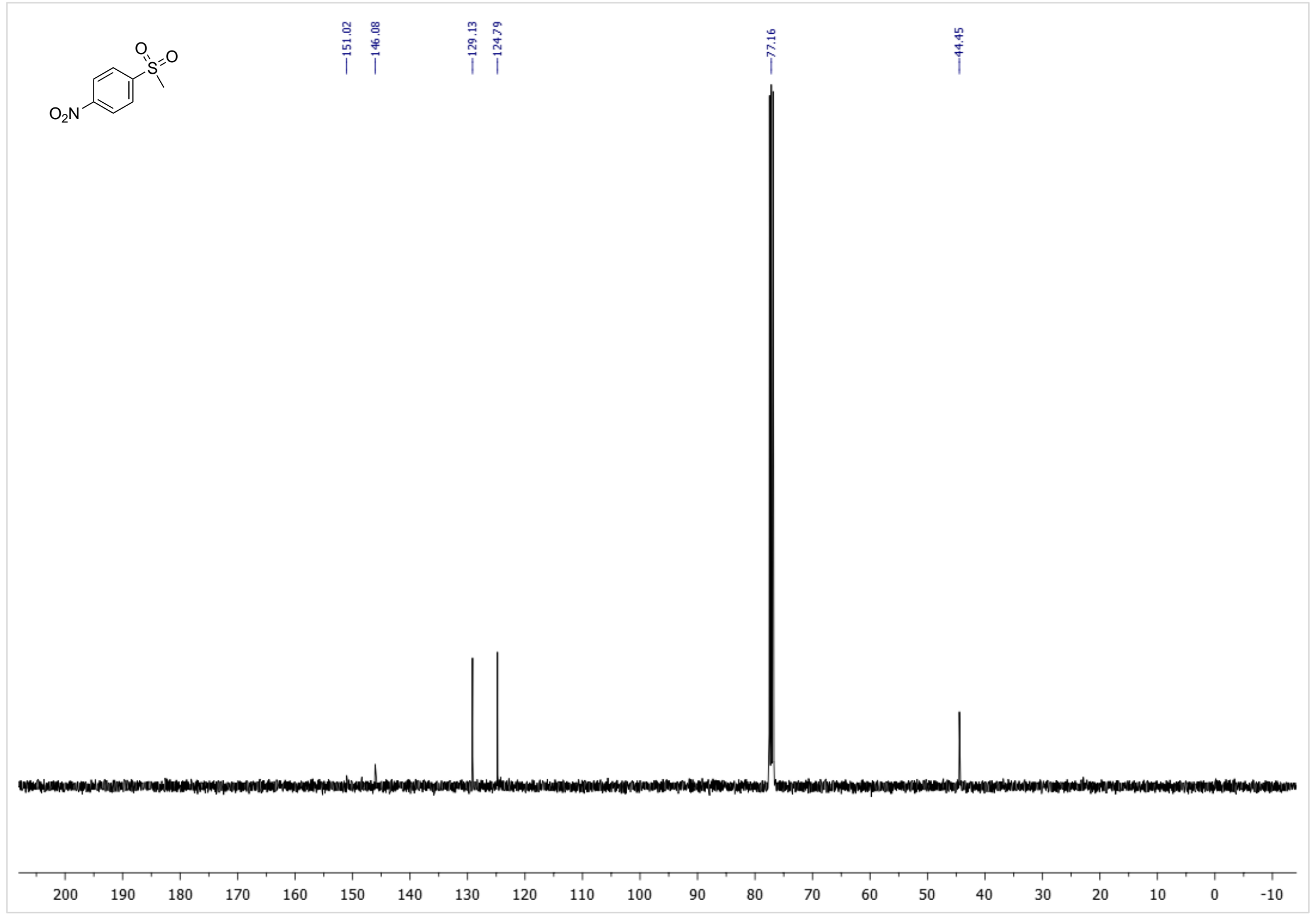

S84 
1-methyl-3-(methylsulfonyl)benzene (3j)

${ }^{1} \mathrm{H}$ NMR $\left(500 \mathrm{MHz}, \mathrm{CDCl}_{3}, 298 \mathrm{~K}\right)$

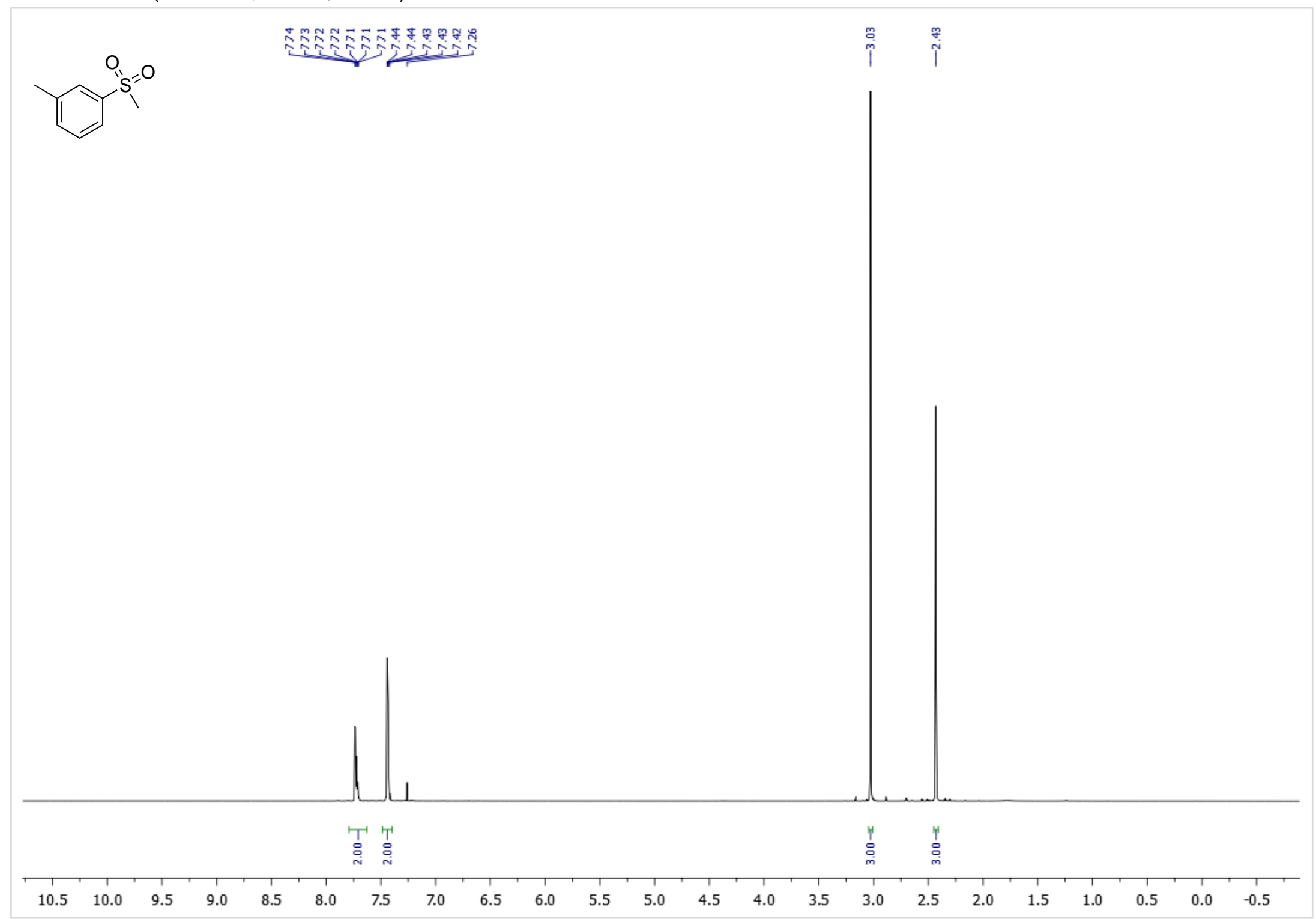


${ }^{13} \mathrm{C}\left\{{ }^{1} \mathrm{H}\right\}$ NMR $\left(126 \mathrm{MHz}, \mathrm{CDCl}_{3}, 298 \mathrm{~K}\right)$

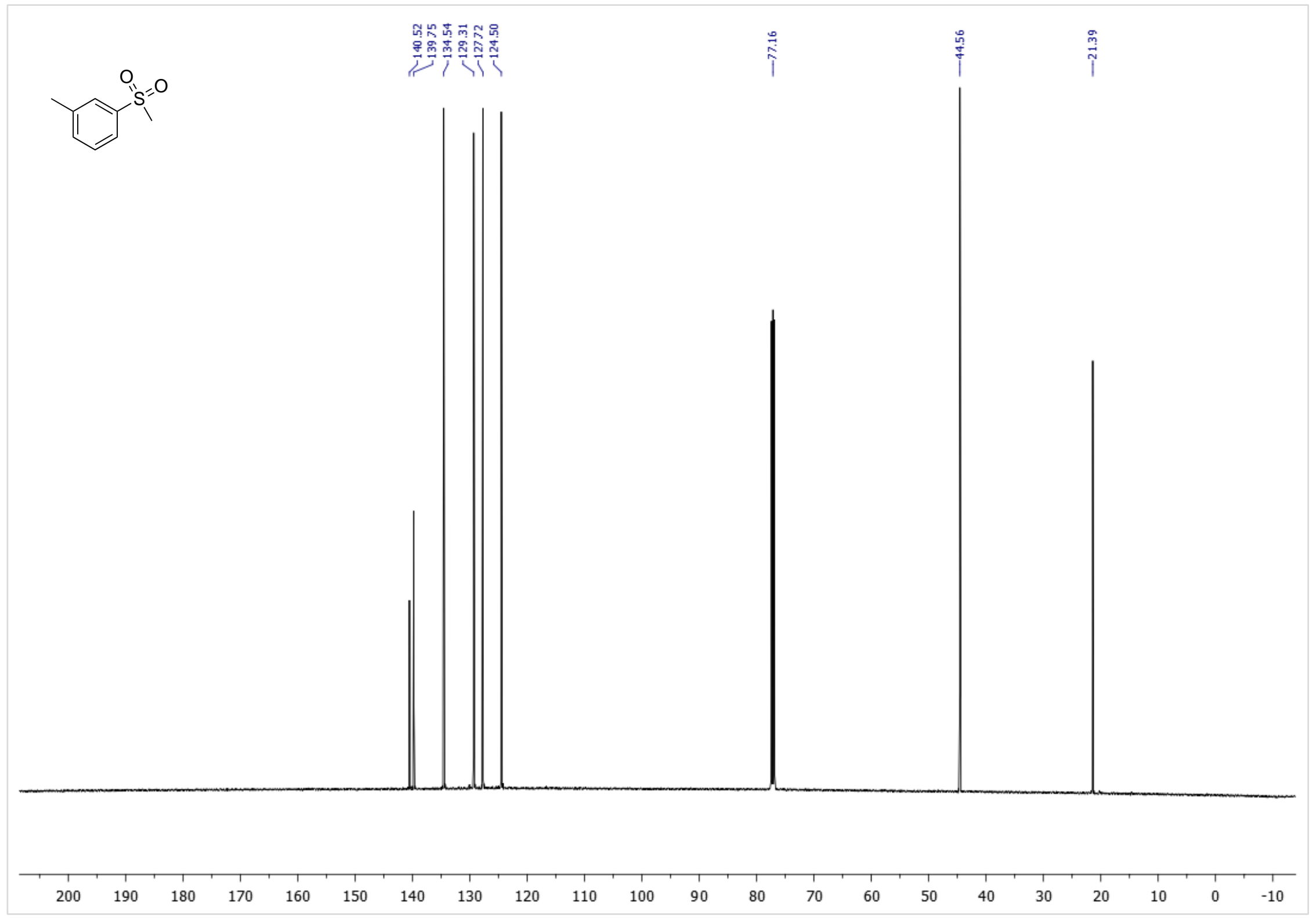

S86 
1-bromo-3-(methylsulfonyl)benzene (3k)

${ }^{1} \mathbf{H}$ NMR $\left(500 \mathrm{MHz}, \mathrm{CDCl}_{3}, 298 \mathrm{~K}\right)$

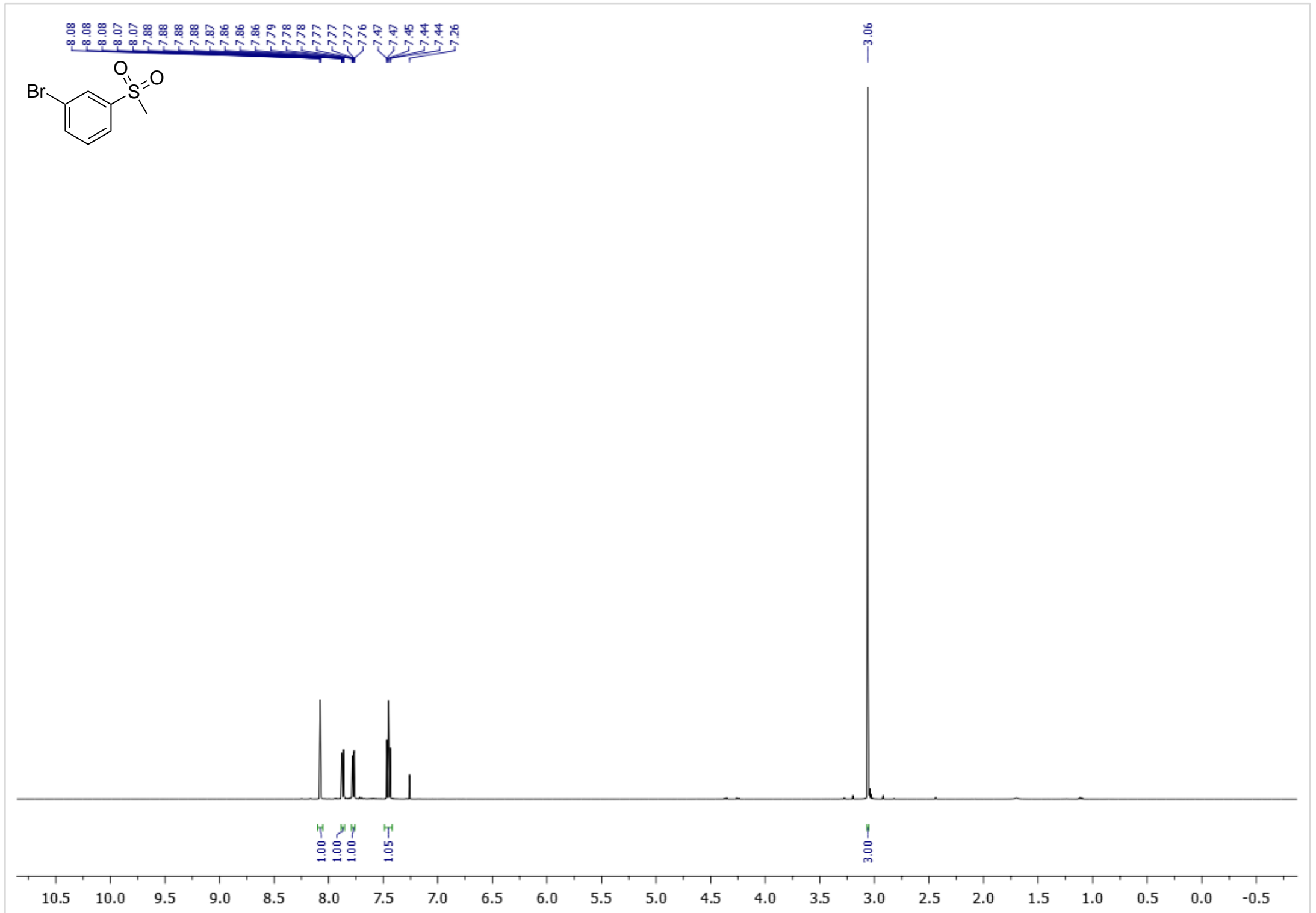


${ }^{13} \mathbf{C}\left\{{ }^{1} \mathrm{H}\right\}$ NMR $\left(126 \mathrm{MHz}, \mathrm{CDCl}_{3}, 298 \mathrm{~K}\right)$

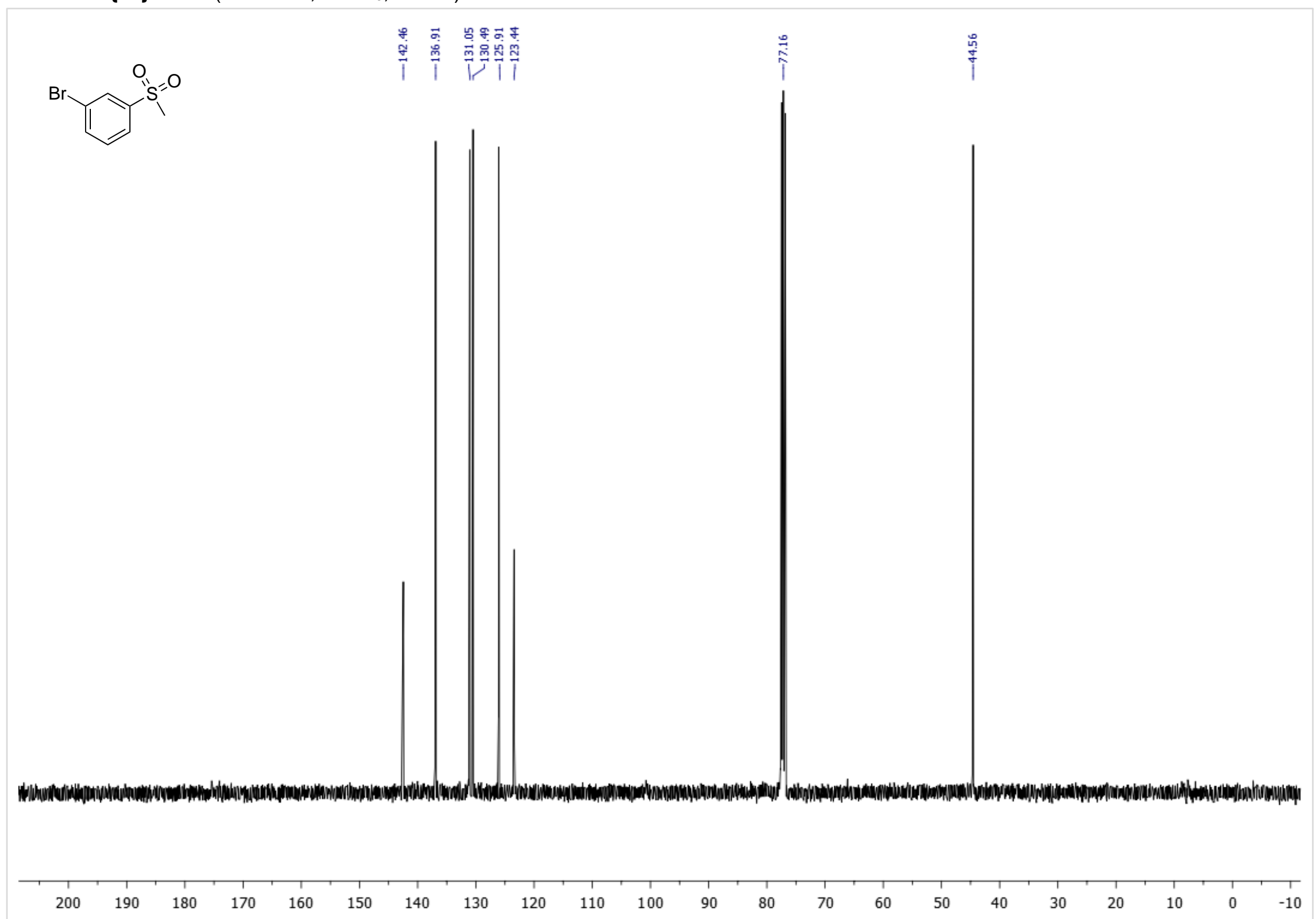


1-chloro-3-(methylsulfonyl)benzene (3I)

${ }^{1} \mathbf{H}$ NMR $\left(500 \mathrm{MHz}, \mathrm{CDCl}_{3}, 298 \mathrm{~K}\right)$

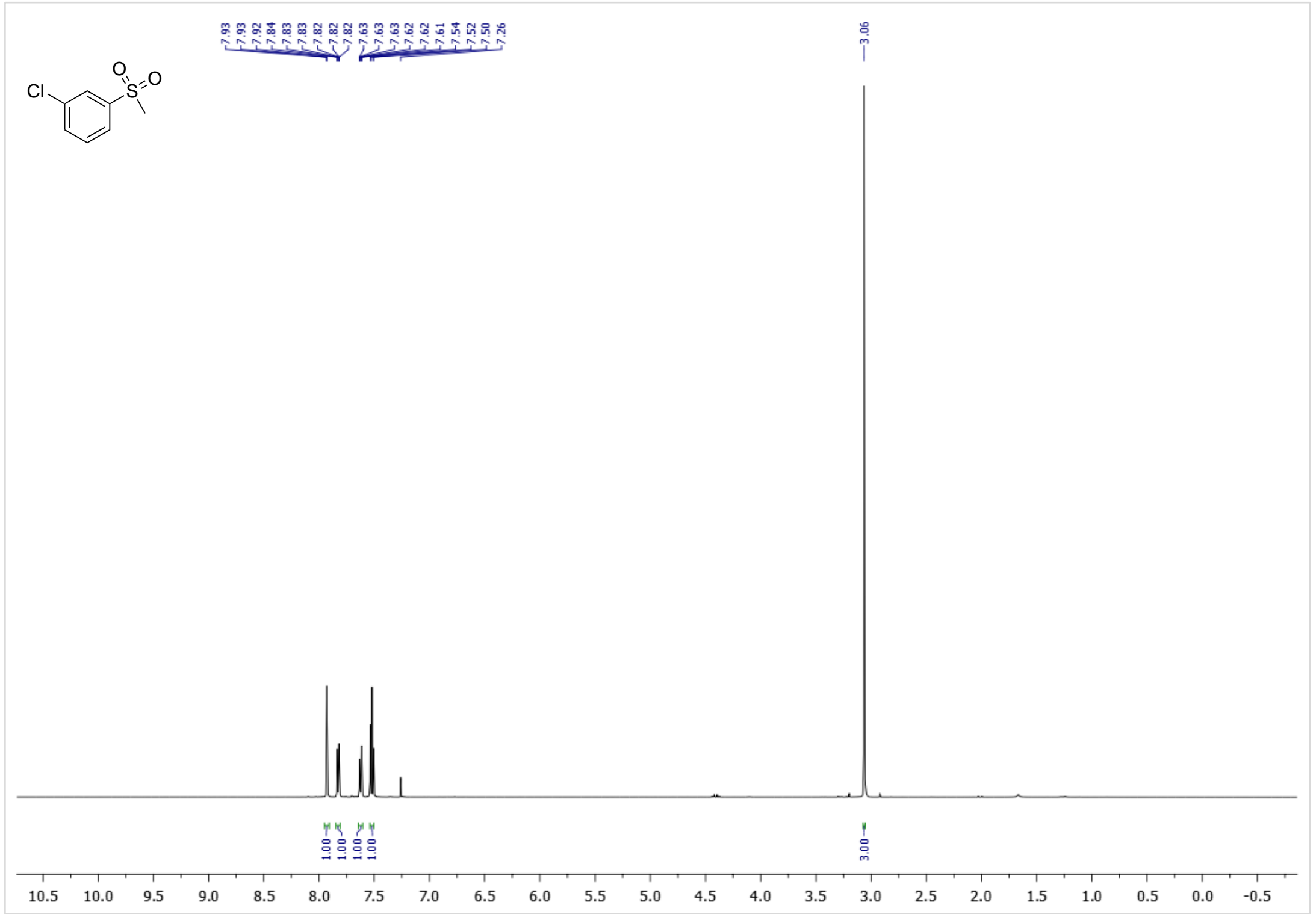


${ }^{13} \mathrm{C}\left\{{ }^{1} \mathrm{H}\right\}$ NMR $\left(126 \mathrm{MHz}, \mathrm{CDCl}_{3}, 298 \mathrm{~K}\right)$

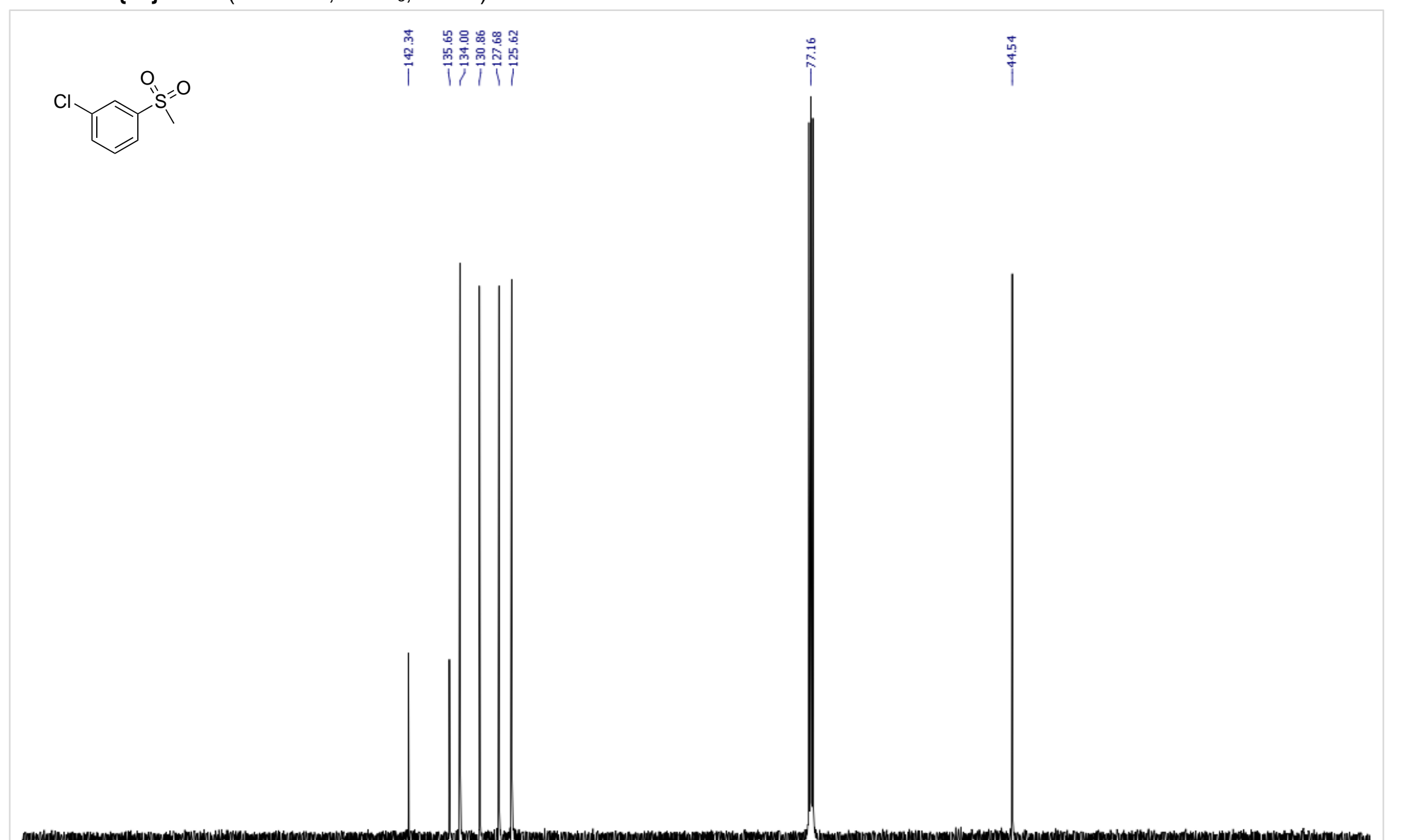

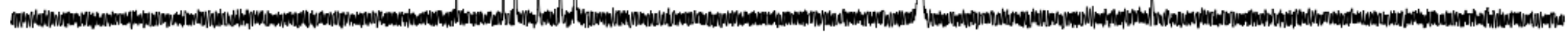


1-bromo-2-(methylsulfinyl)benzene (3m)

${ }^{1} \mathbf{H}$ NMR $\left(500 \mathrm{MHz}, \mathrm{CDCl}_{3}, 298 \mathrm{~K}\right)$

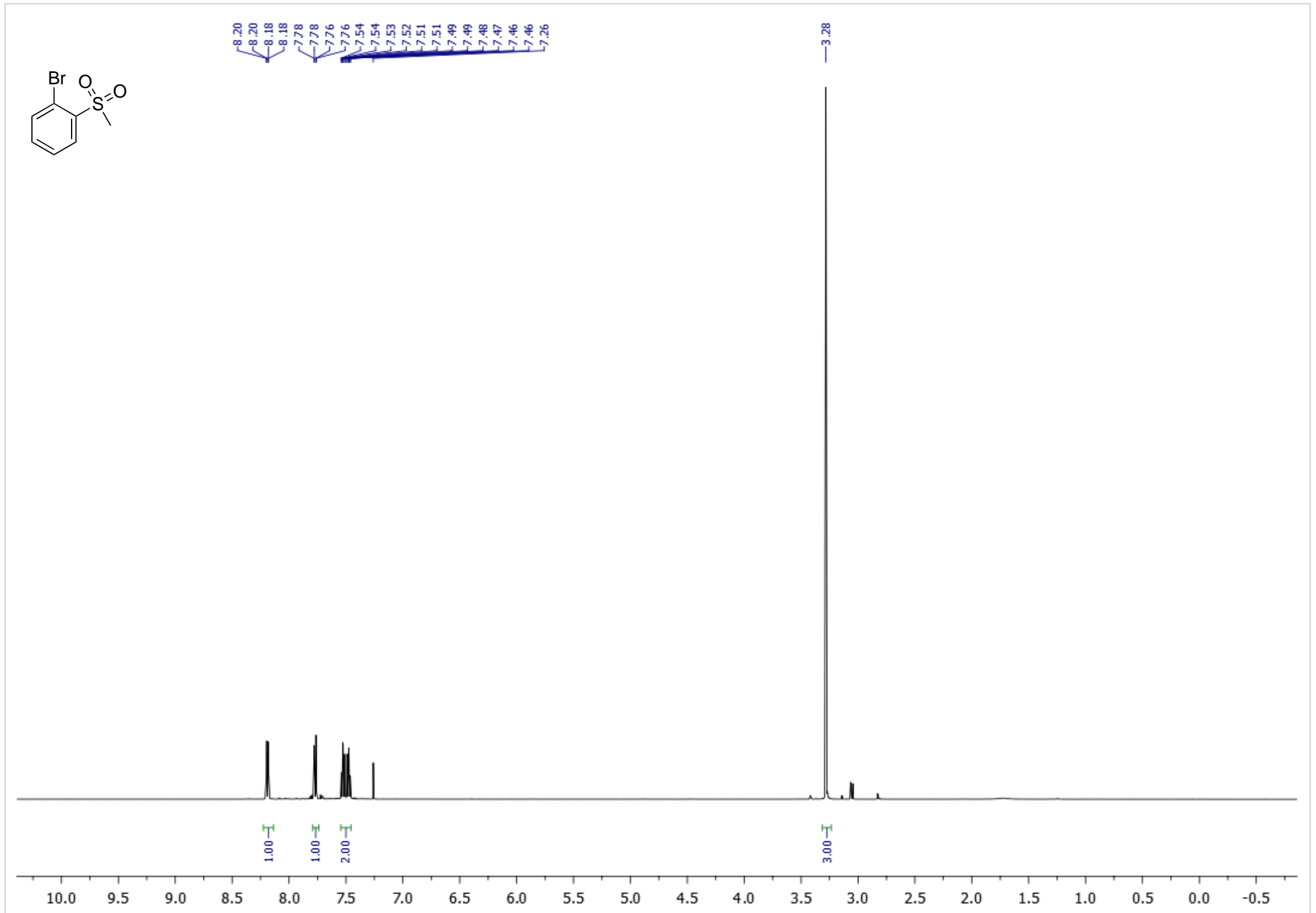


${ }^{13} \mathrm{C}\left\{{ }^{1} \mathrm{H}\right\}$ NMR $\left(126 \mathrm{MHz}, \mathrm{CDCl}_{3}, 298 \mathrm{~K}\right)$

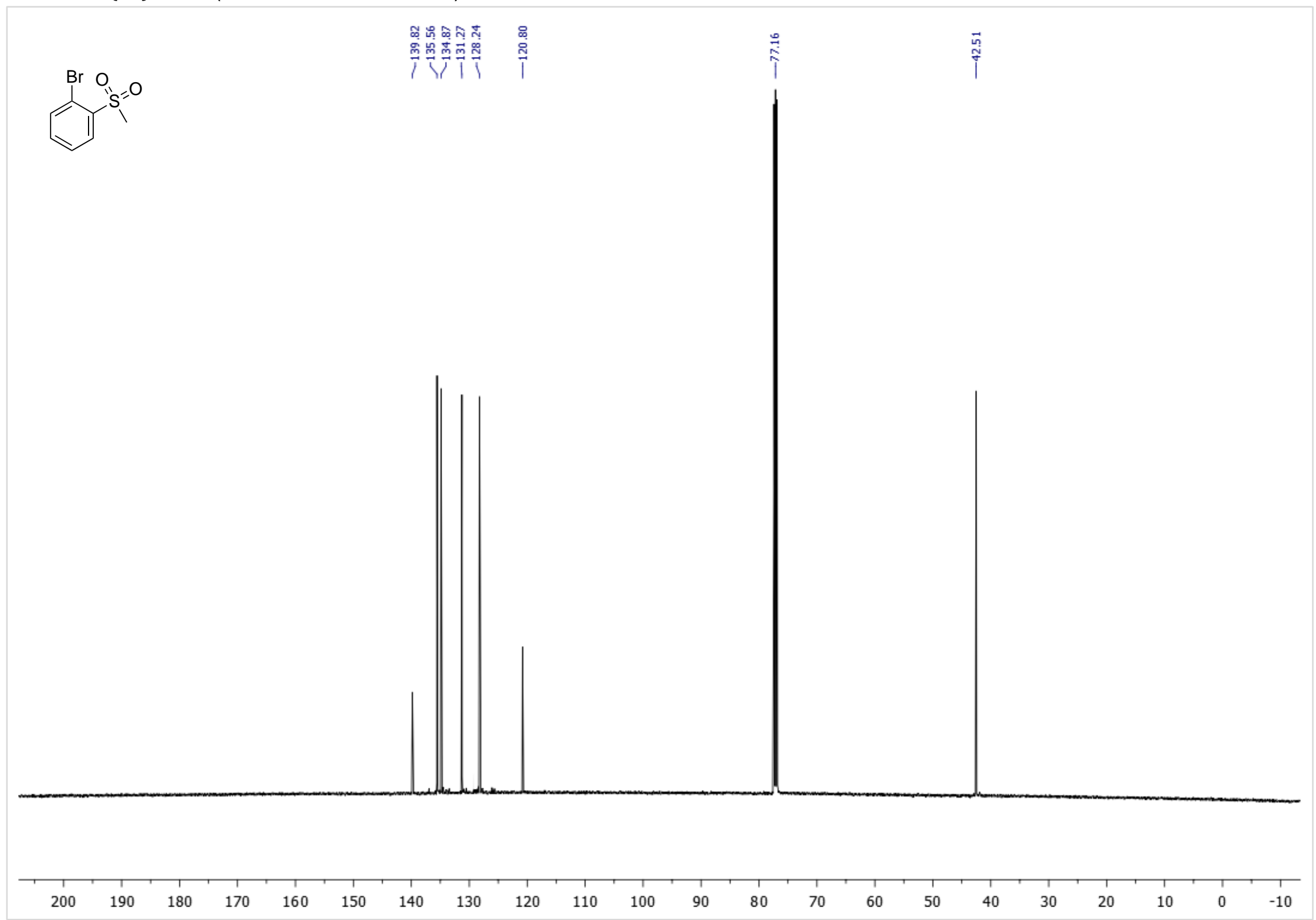


1-chloro-2-(methylsulfinyl)benzene (3n)

${ }^{1} \mathbf{H}$ NMR $\left(500 \mathrm{MHz}, \mathrm{CDCl}_{3}, 298 \mathrm{~K}\right)$

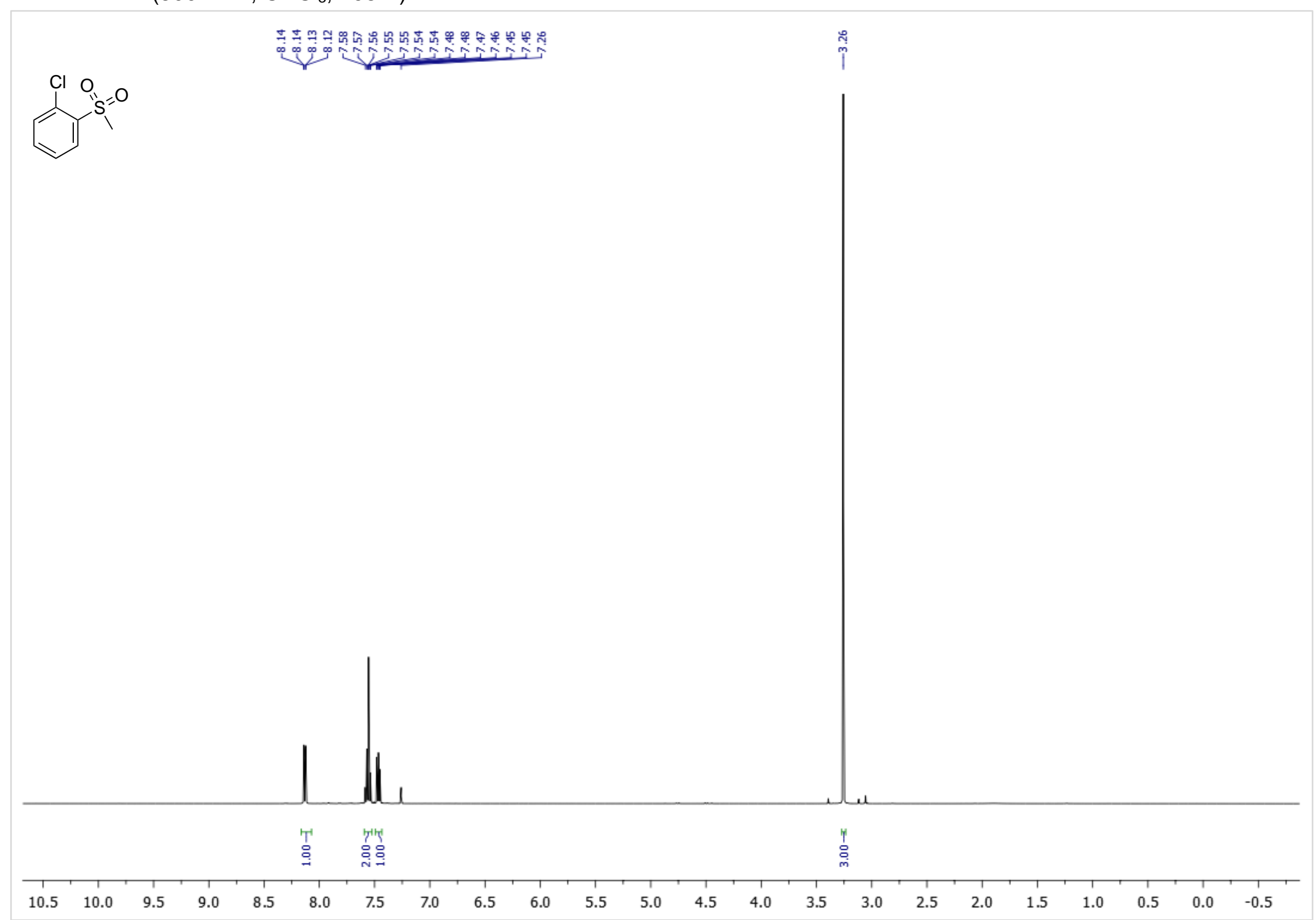


${ }^{13} \mathrm{C}\left\{{ }^{1} \mathrm{H}\right\}$ NMR $\left(126 \mathrm{MHz}, \mathrm{CDCl}_{3}, 298 \mathrm{~K}\right)$

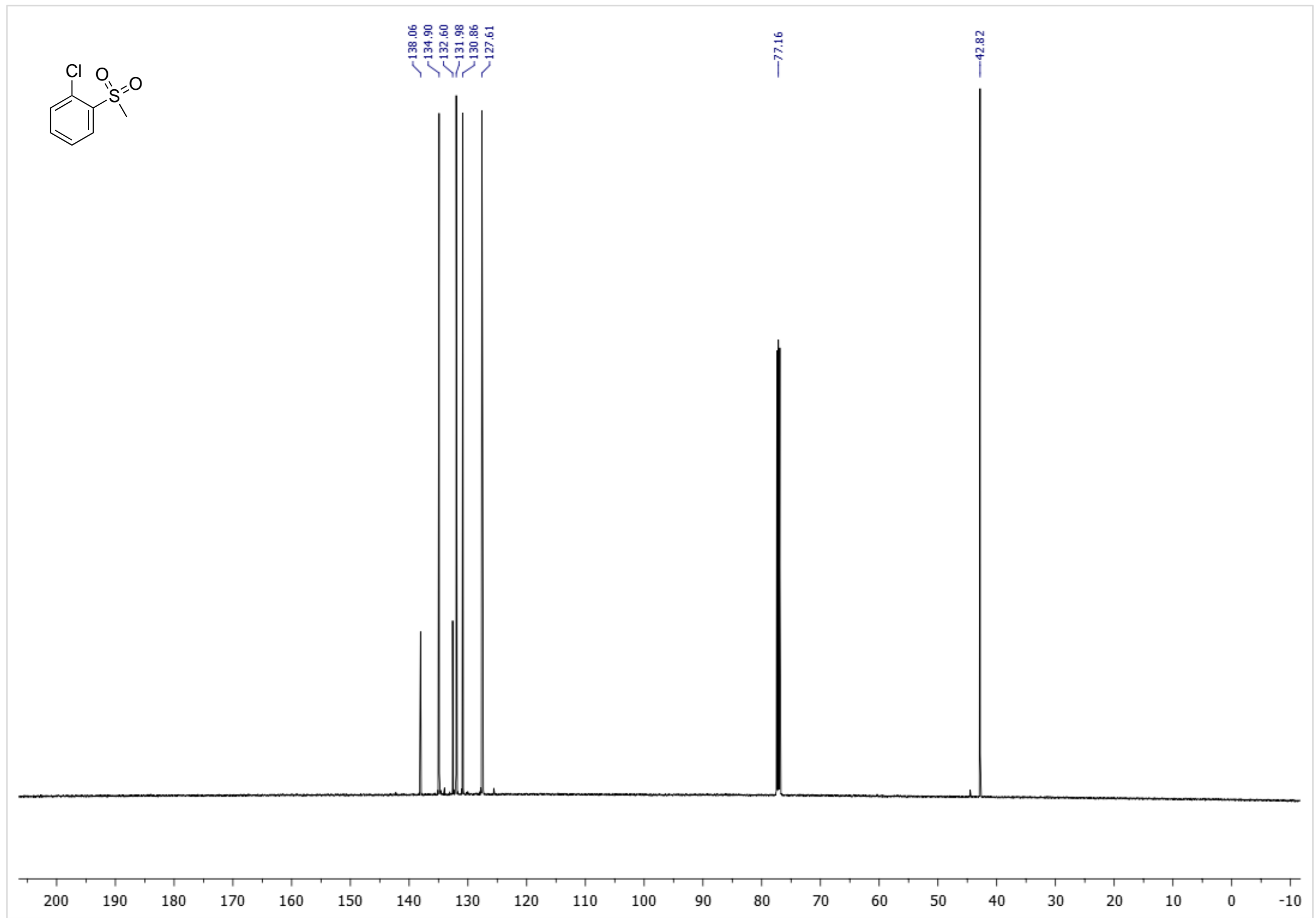


1-iodo-2-(methylsulfinyl)benzene (30)

${ }^{1} \mathbf{H}$ NMR $\left(500 \mathrm{MHz}, \mathrm{CDCl}_{3}, 298 \mathrm{~K}\right)$

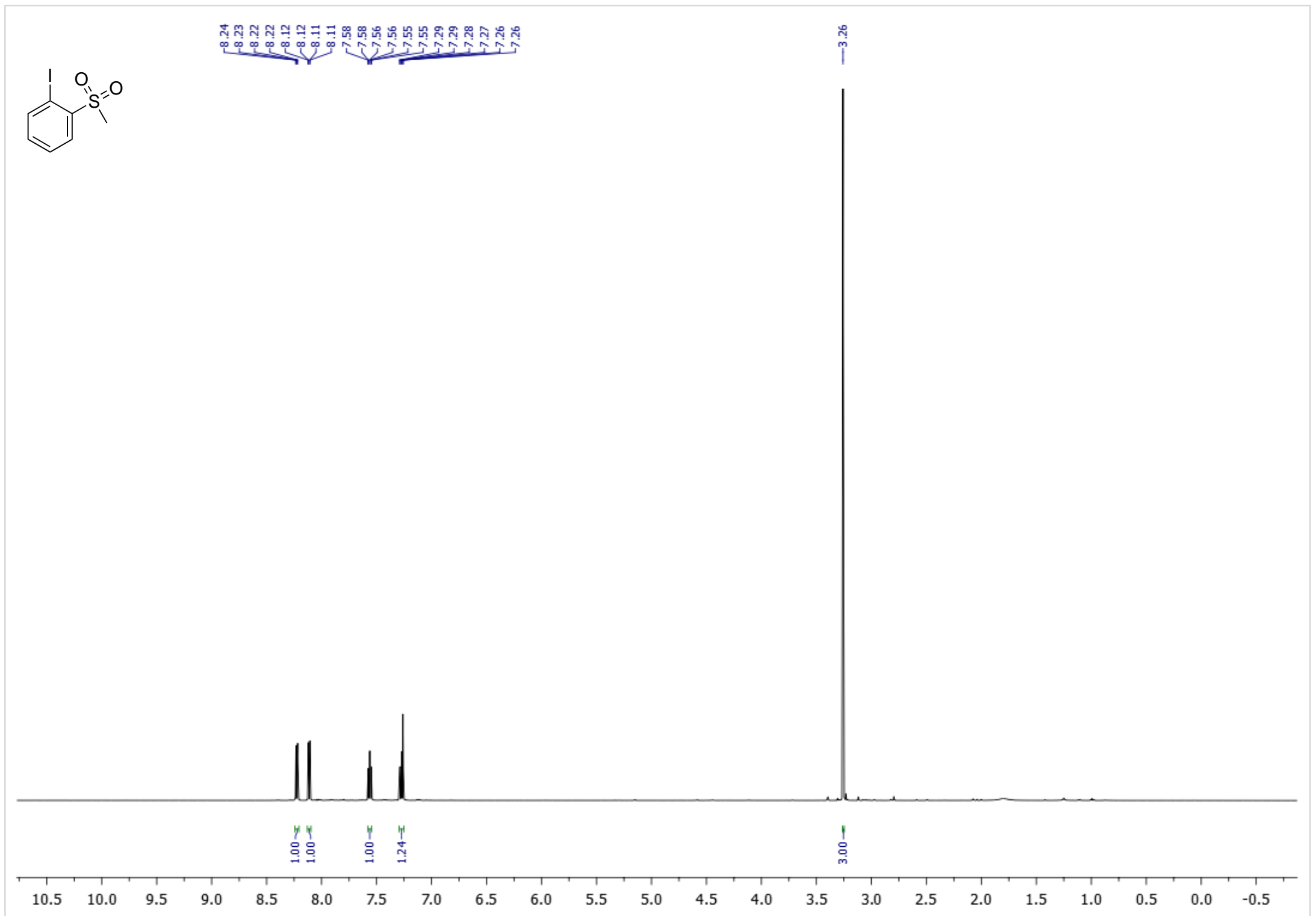


${ }^{13} \mathrm{C}\left\{{ }^{1} \mathrm{H}\right\}$ NMR $\left(126 \mathrm{MHz}, \mathrm{CDCl}_{3}, 298 \mathrm{~K}\right)$

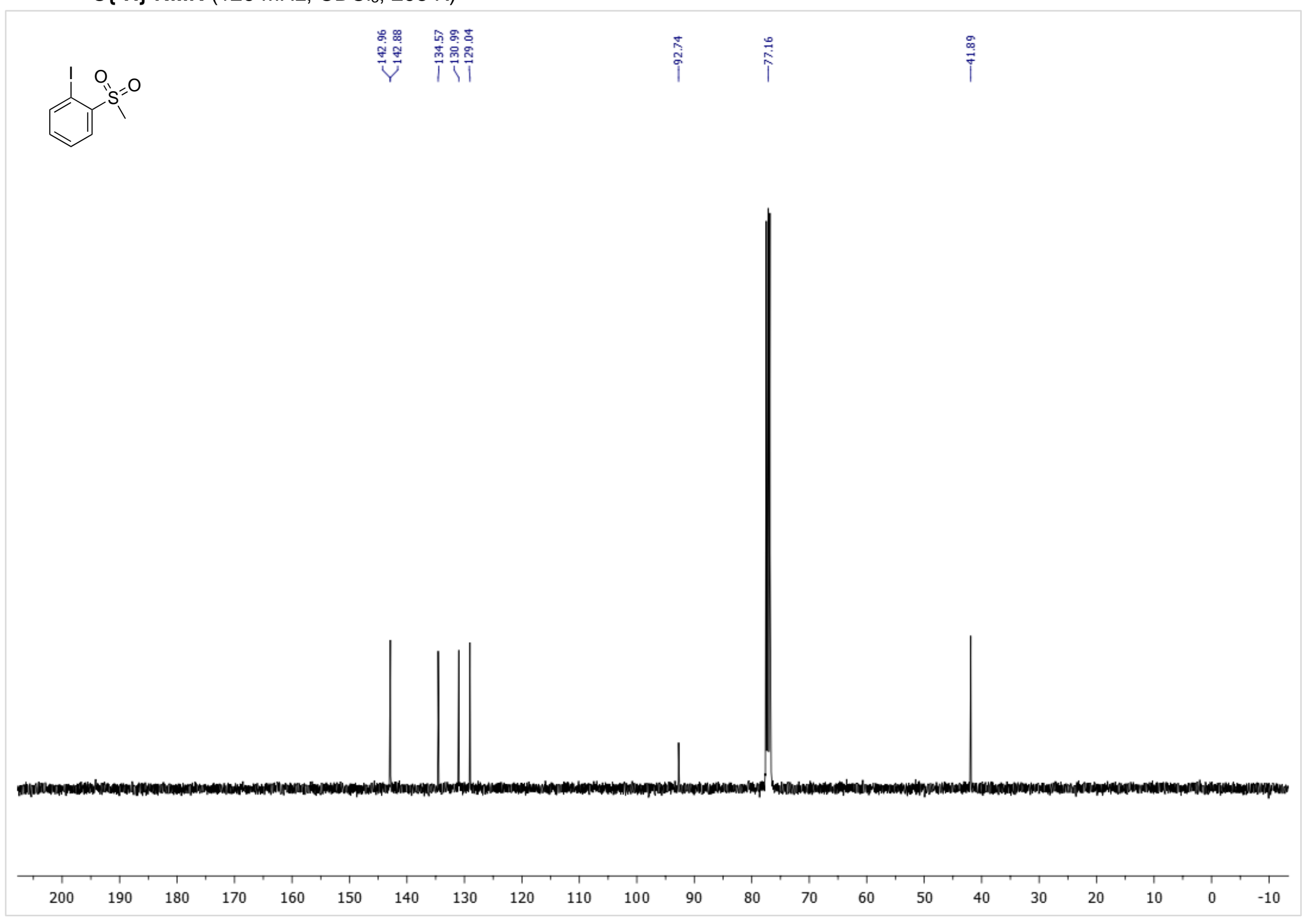


1,3-dichloro-5-(methylsulfinyl)benzene (3p)

${ }^{1} \mathrm{H}$ NMR $\left(500 \mathrm{MHz}, \mathrm{CDCl}_{3}, 298 \mathrm{~K}\right)$

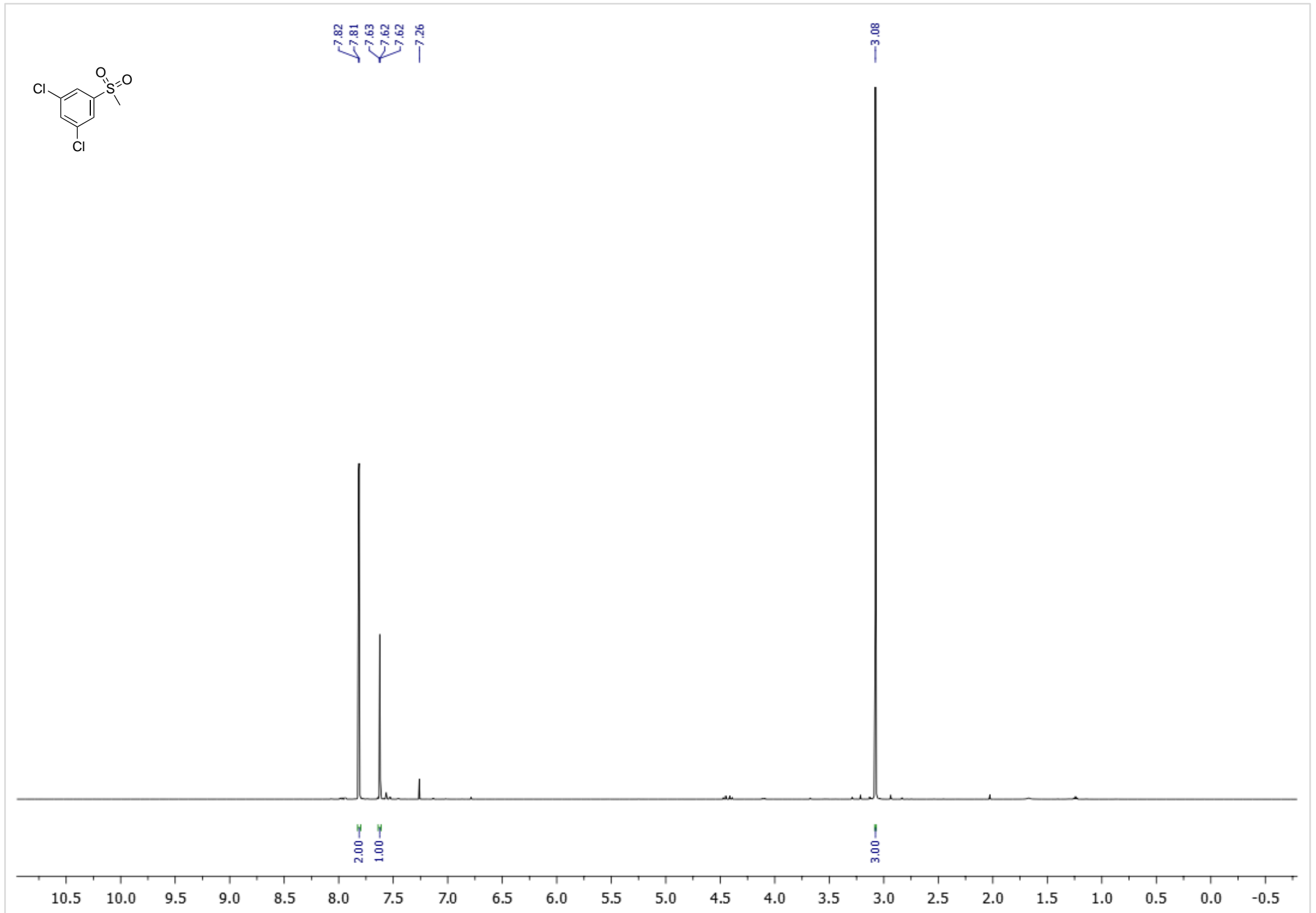


${ }^{13} \mathrm{C}\left\{{ }^{1} \mathrm{H}\right\}$ NMR $\left(126 \mathrm{MHz}, \mathrm{CDCl}_{3}, 298 \mathrm{~K}\right)$

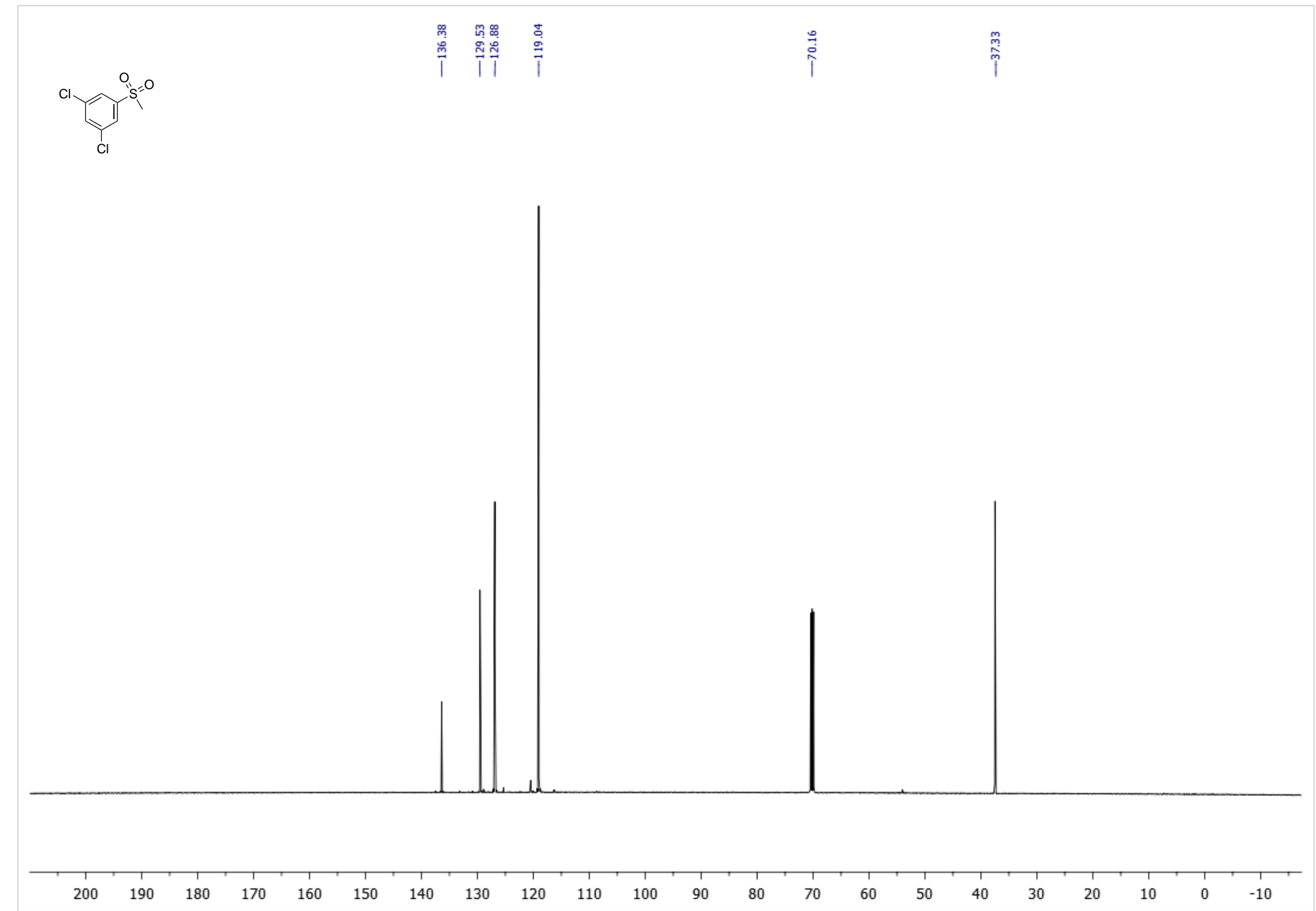


${ }^{1} \mathbf{H}$ NMR (500 MHz, $\left.\mathrm{CDCl}_{3}, 298 \mathrm{~K}\right)$

(ethylsulfinyl)benzene (3q)

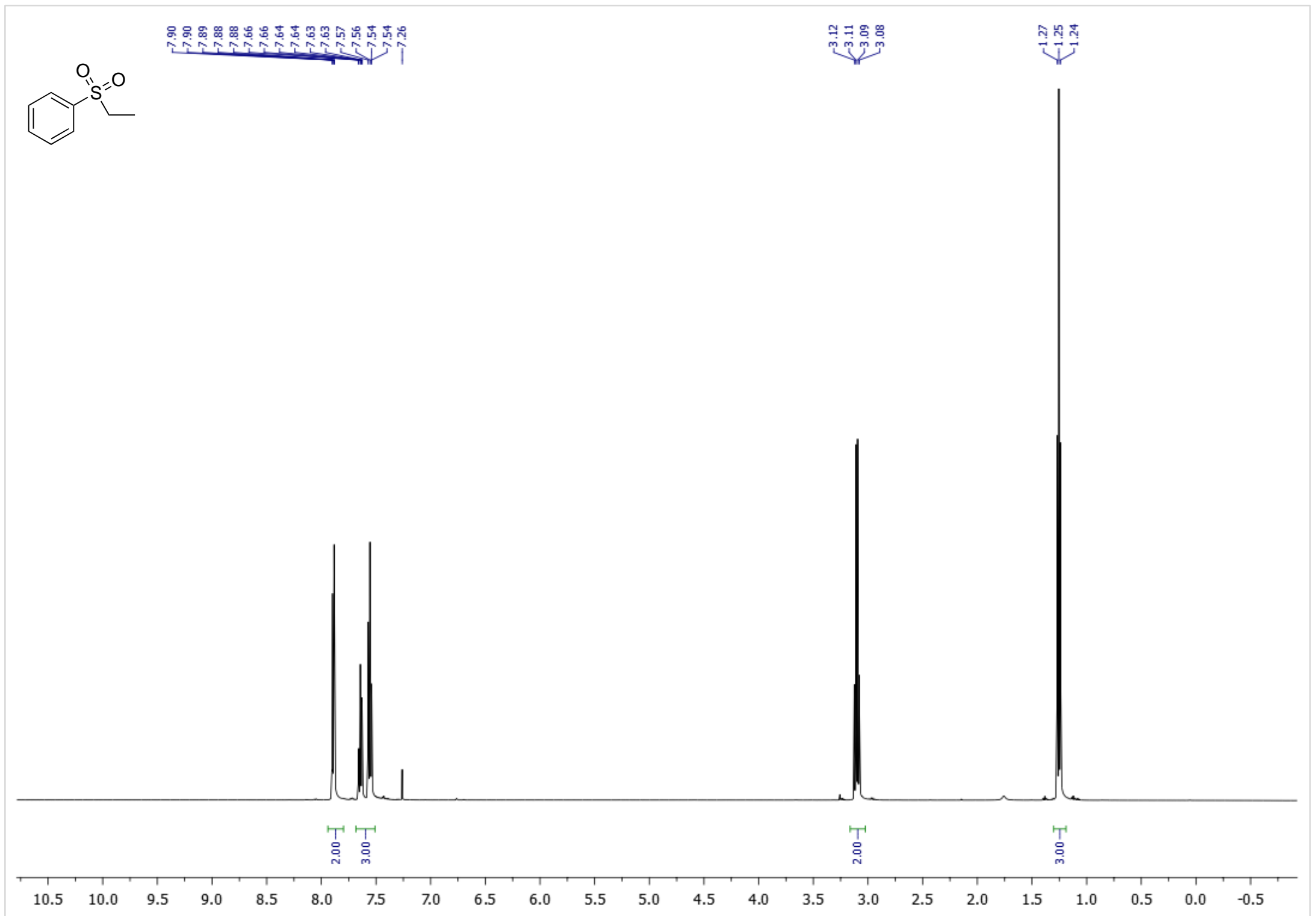


${ }^{13} \mathrm{C}\left\{{ }^{1} \mathrm{H}\right\}$ NMR $\left(126 \mathrm{MHz}, \mathrm{CDCl}_{3}, 298 \mathrm{~K}\right)$

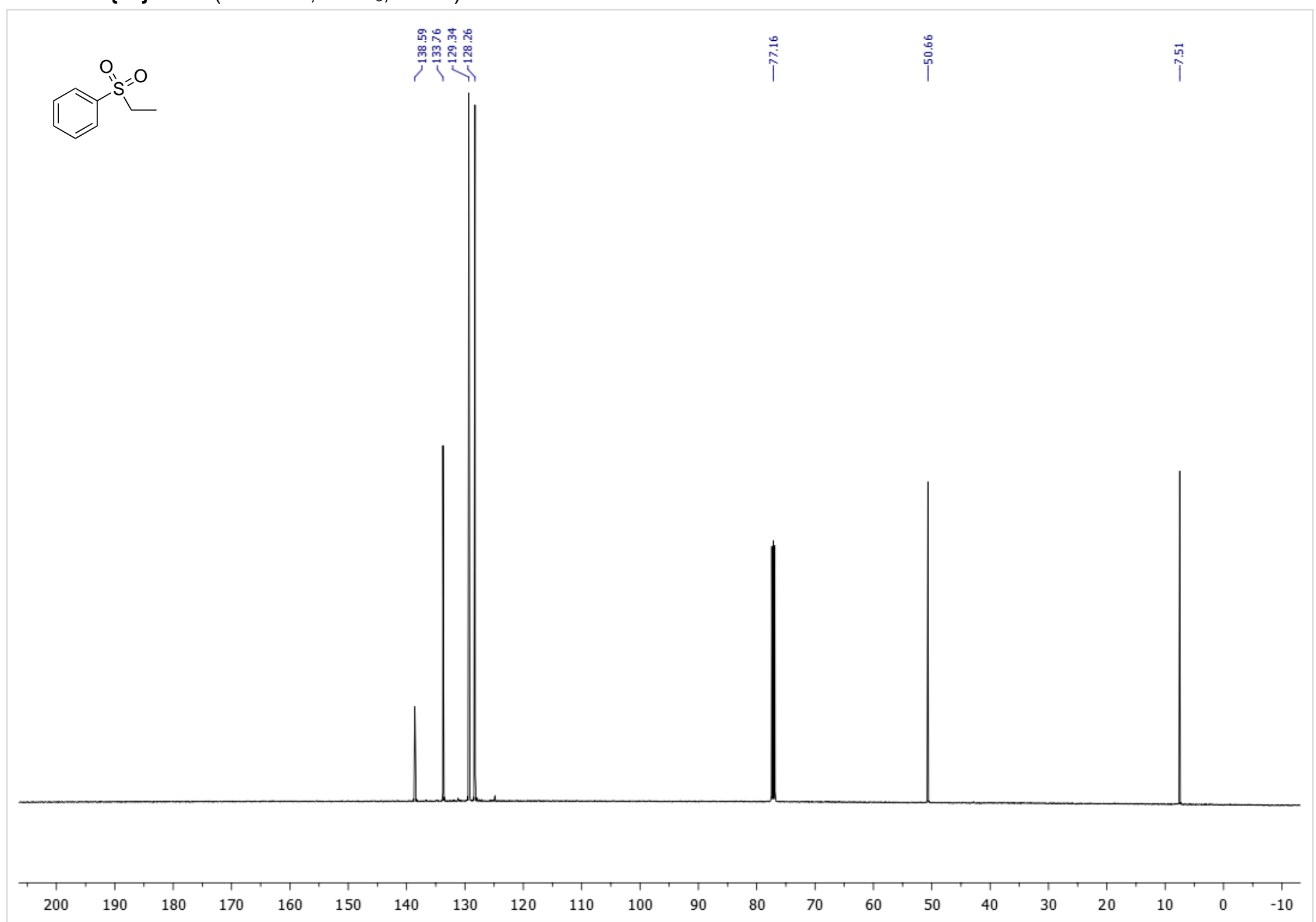


${ }^{1} \mathbf{H}$ NMR $\left(500 \mathrm{MHz}, \mathrm{CDCl}_{3}, 298 \mathrm{~K}\right)$

(propylsulfonyl)benzene (3r)

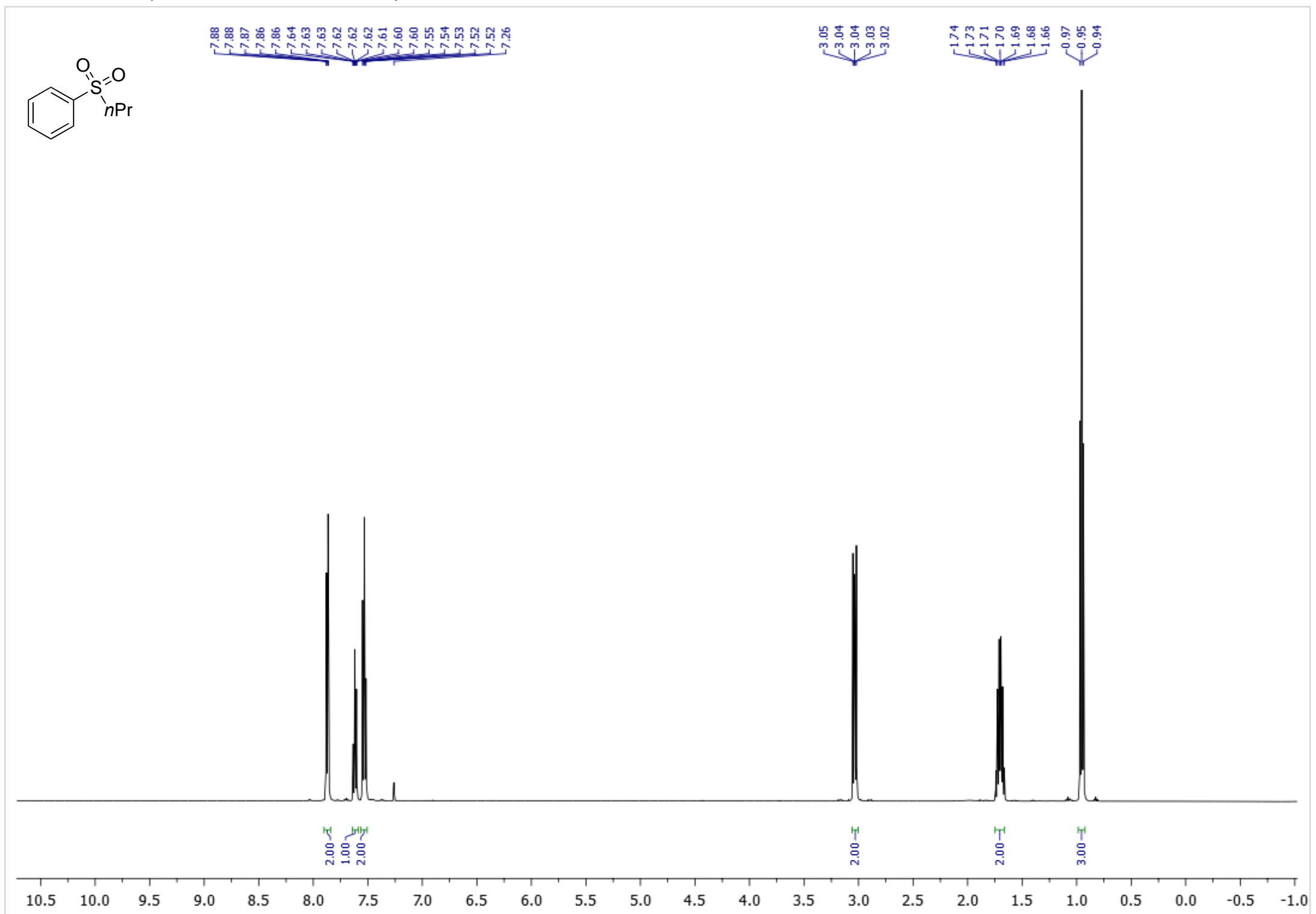

S101 
${ }^{13} \mathrm{C}\left\{{ }^{1} \mathrm{H}\right\}$ NMR $\left(126 \mathrm{MHz}, \mathrm{CDCl}_{3}, 298 \mathrm{~K}\right)$

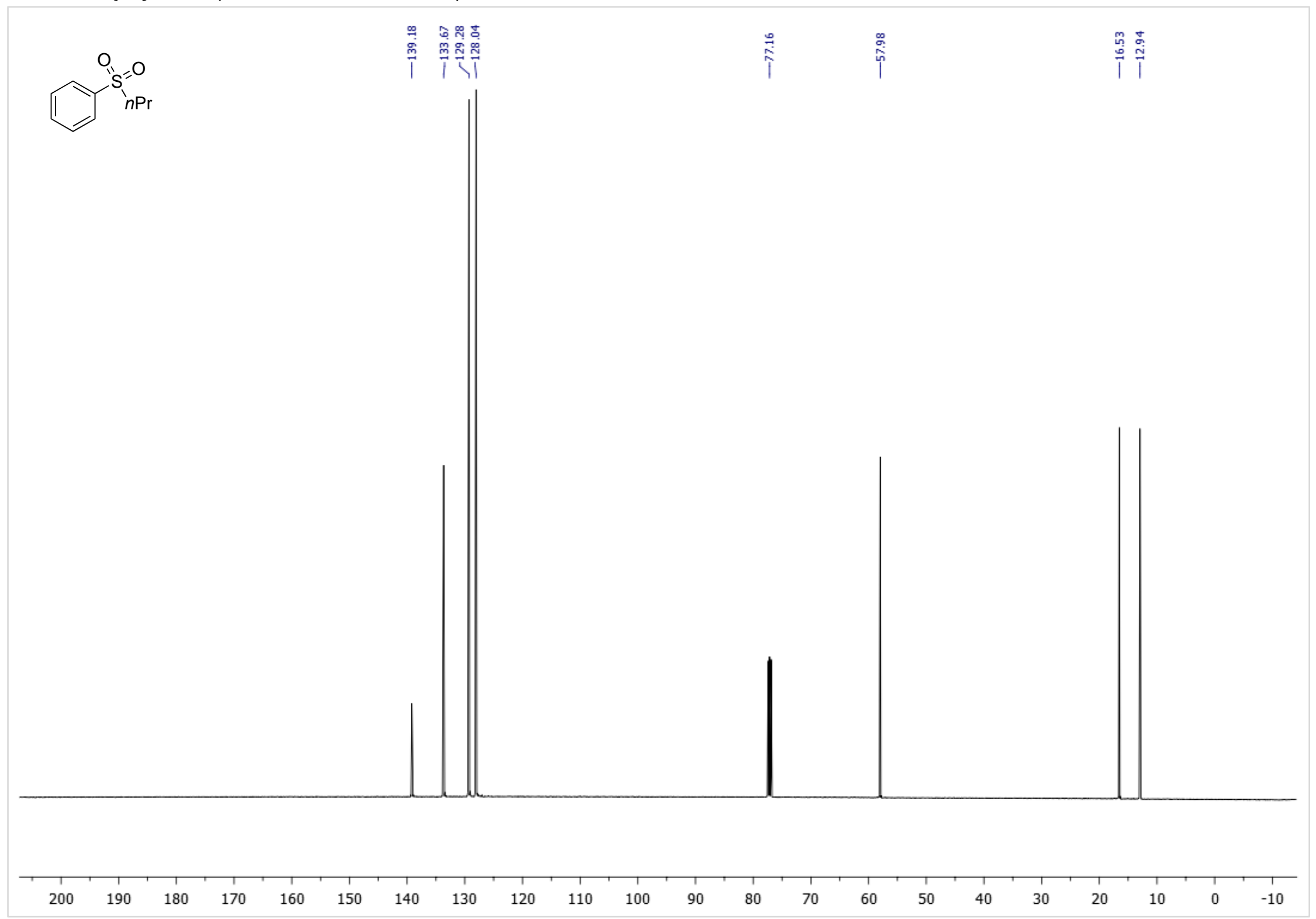


${ }^{1} \mathbf{H}$ NMR $\left(500 \mathrm{MHz}, \mathrm{CDCl}_{3}, 298 \mathrm{~K}\right)$

(cyclopropylsulfonyl)benzene (3s)

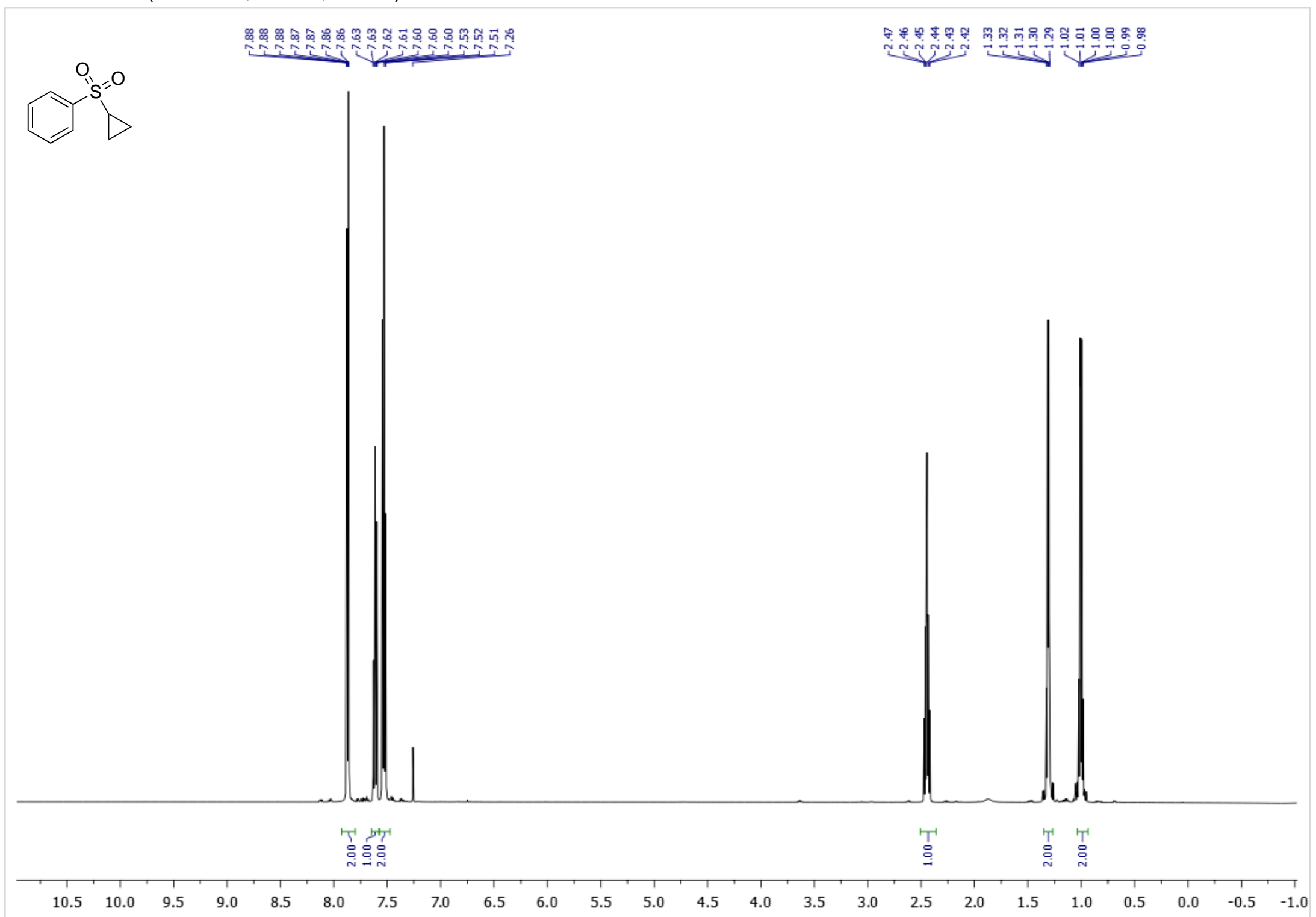

S103 
${ }^{13} \mathrm{C}\left\{{ }^{1} \mathrm{H}\right\}$ NMR $\left(126 \mathrm{MHz}, \mathrm{CDCl}_{3}, 298 \mathrm{~K}\right)$

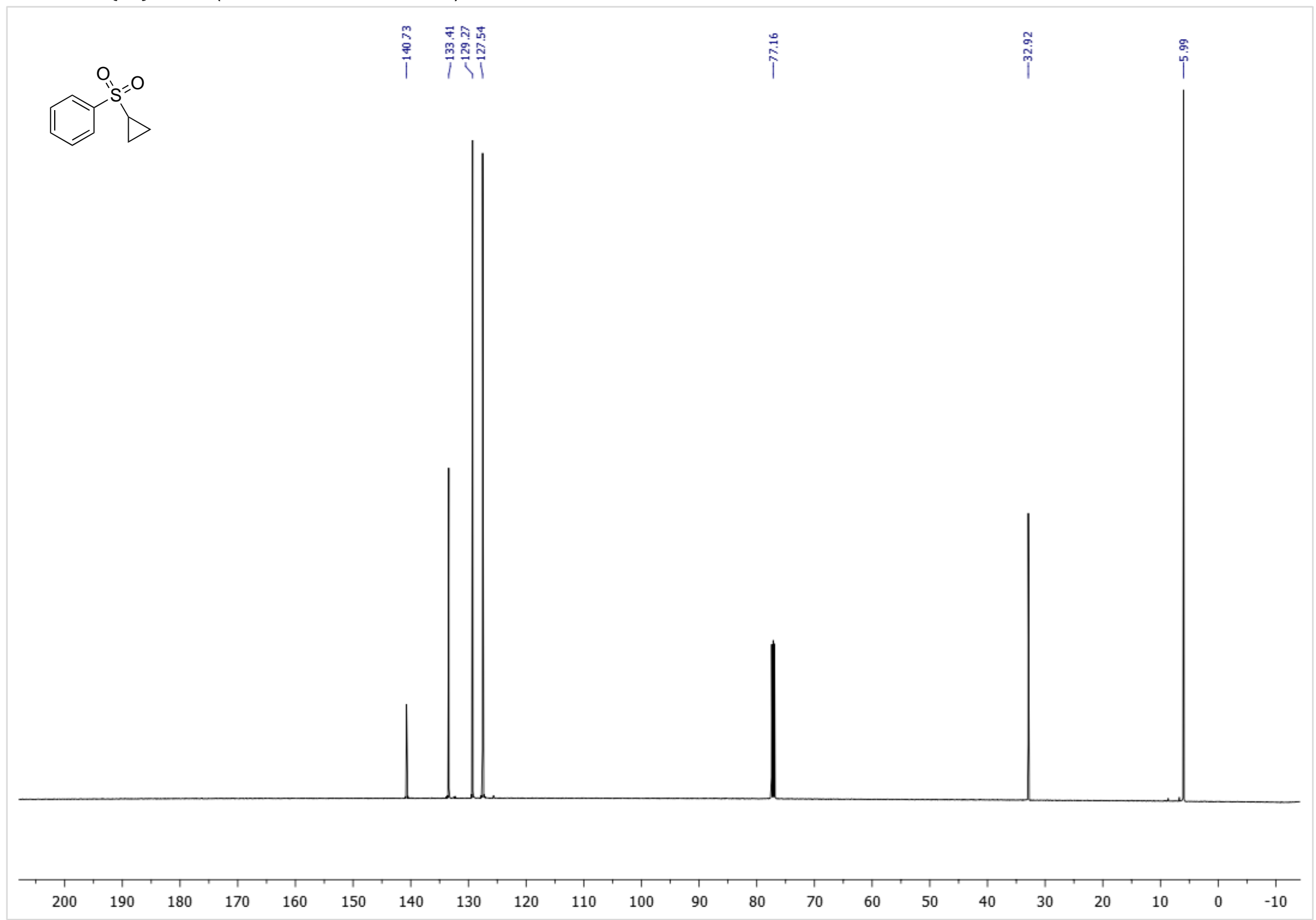


${ }^{\mathbf{1}} \mathbf{H}$ NMR $\left(500 \mathrm{MHz}, \mathrm{CDCl}_{3}, 298 \mathrm{~K}\right)$

(allylsulfonyl)benzene (3t)

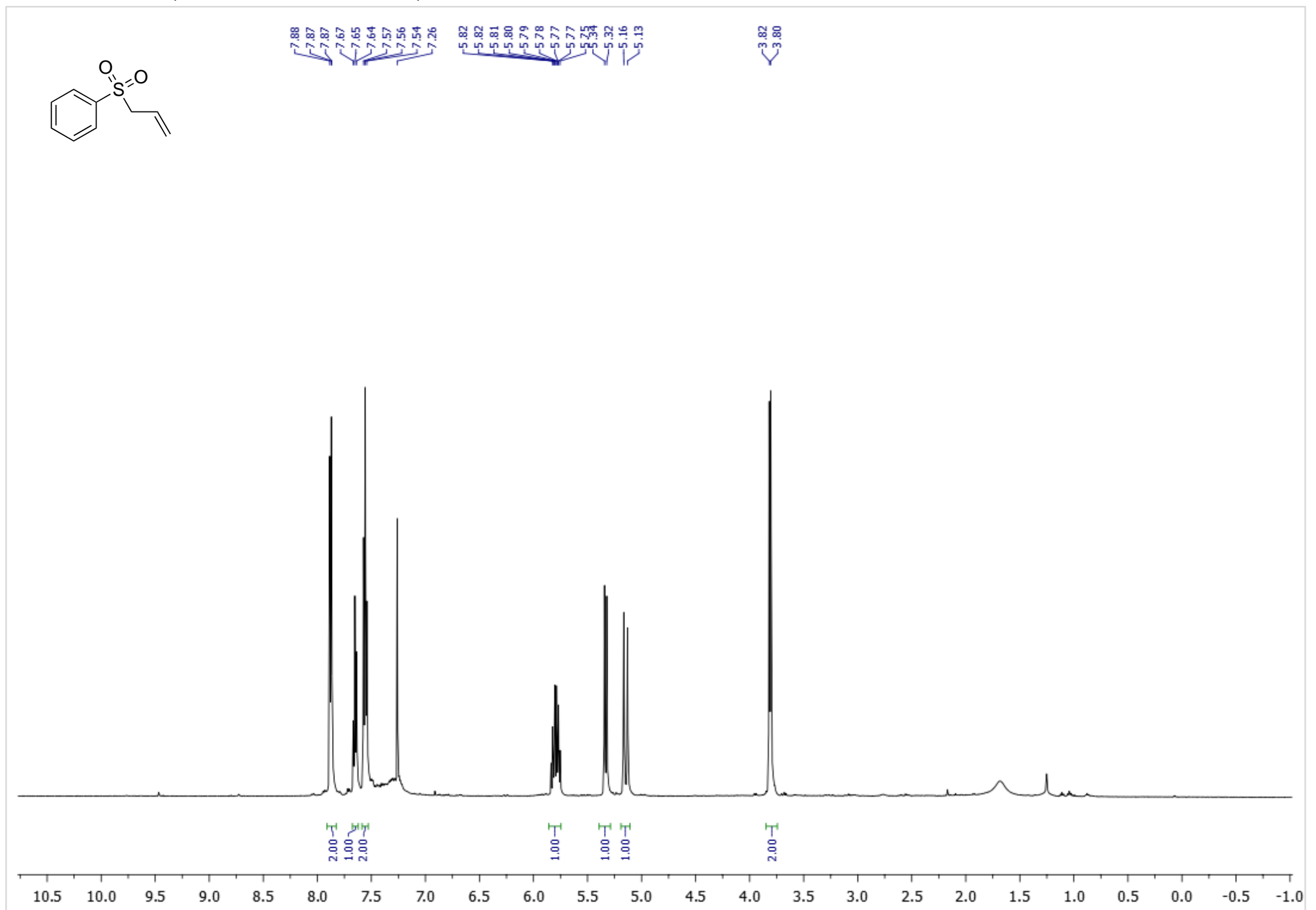

S105 
${ }^{13} \mathrm{C}\left\{{ }^{1} \mathrm{H}\right\}$ NMR $\left(126 \mathrm{MHz}, \mathrm{CDCl}_{3}, 298 \mathrm{~K}\right)$

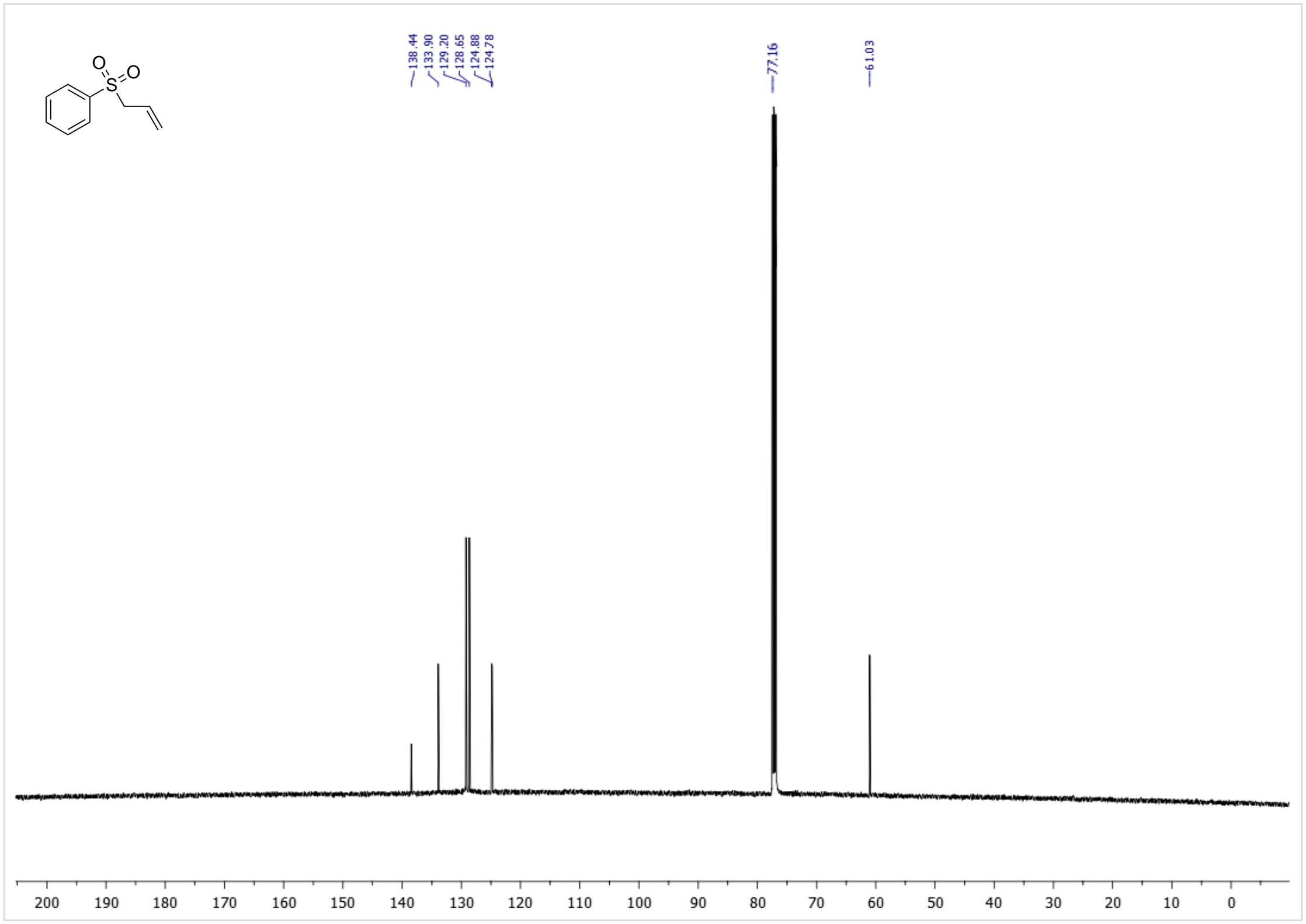

S106 
Sulfonyldibenzene (3v)

${ }^{1} \mathbf{H}$ NMR $\left(400 \mathrm{MHz}, \mathrm{CDCl}_{3}, 298 \mathrm{~K}\right)$
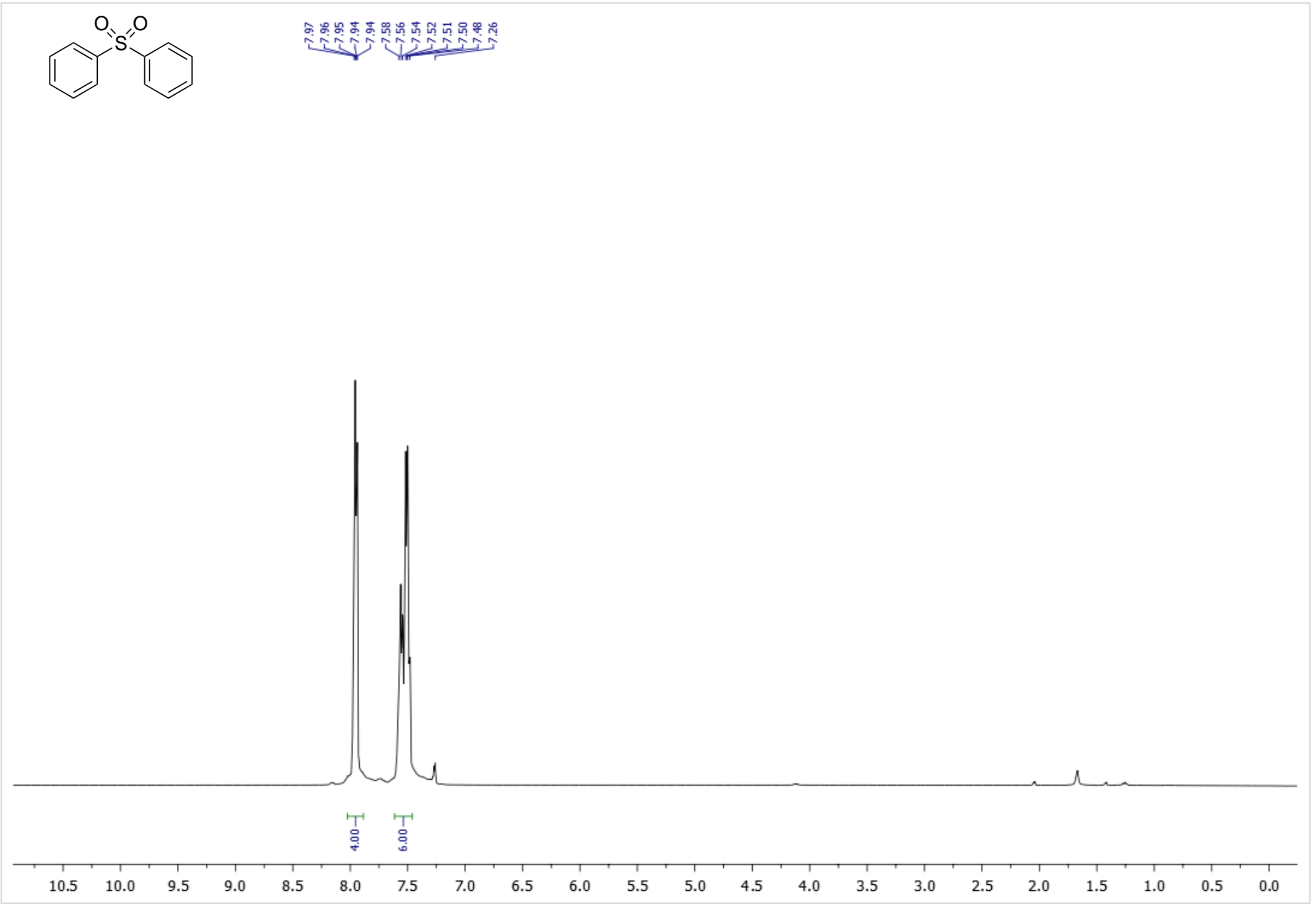

S107 
${ }^{13} \mathrm{C}\left\{{ }^{1} \mathrm{H}\right\}$ NMR $\left(101 \mathrm{MHz}, \mathrm{CDCl}_{3}, 298 \mathrm{~K}\right)$

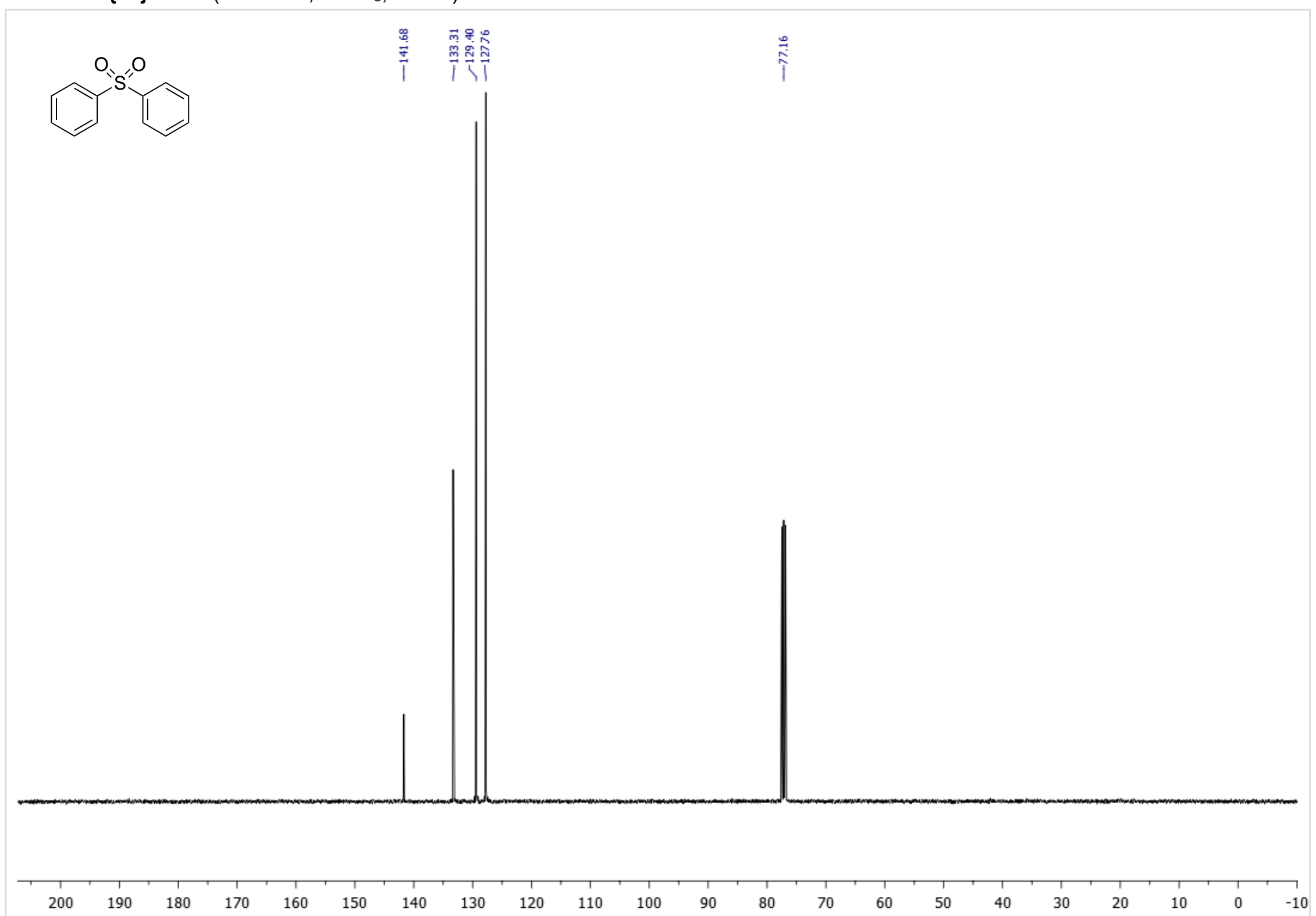


thiochroman-4-one 1,1-dioxide (3w)

${ }^{1} \mathbf{H}$ NMR $\left(500 \mathrm{MHz}, \mathrm{CDCl}_{3}, 298 \mathrm{~K}\right)$

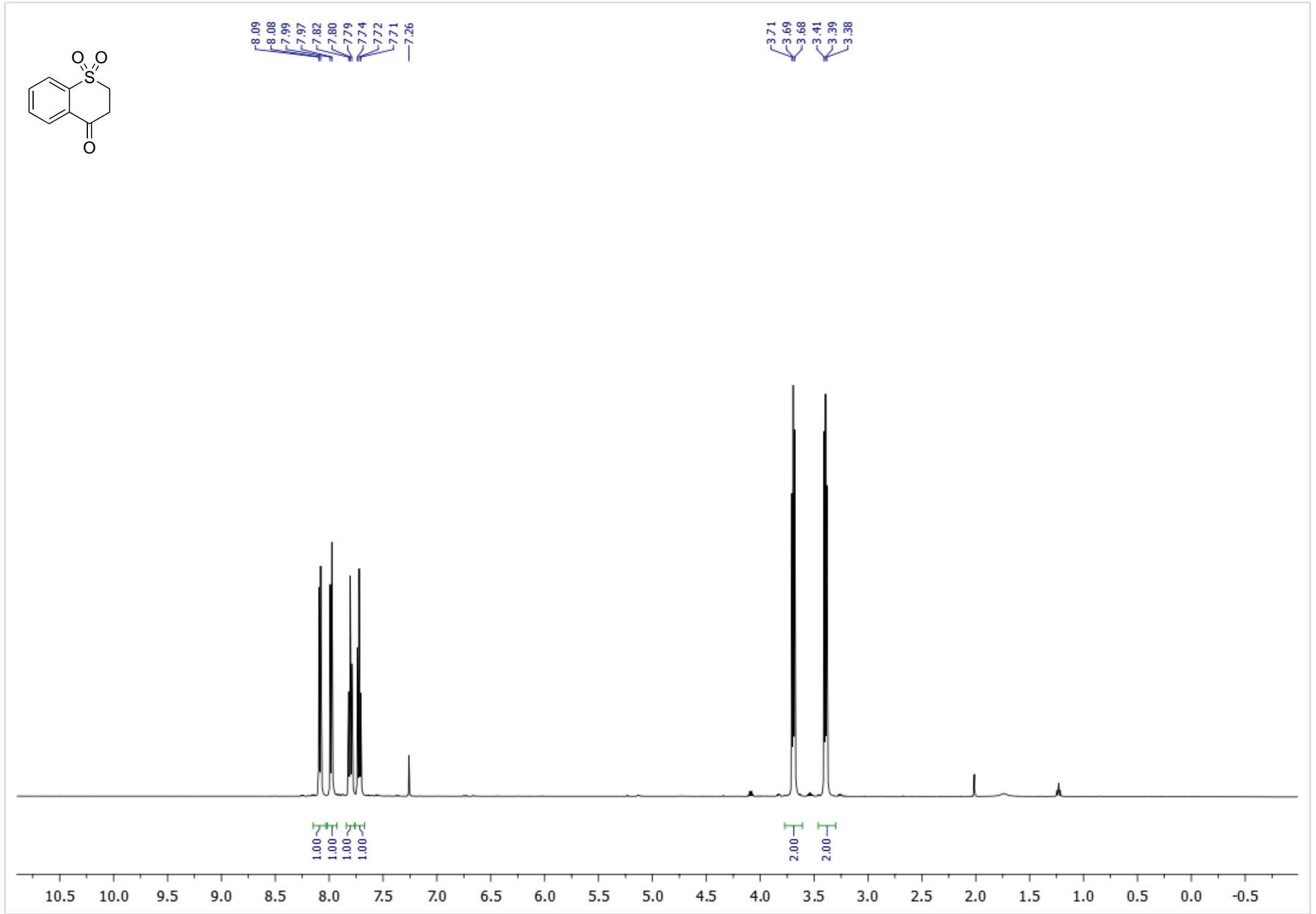

S109 
${ }^{13} \mathrm{C}\left\{{ }^{1} \mathrm{H}\right\}$ NMR $\left(126 \mathrm{MHz}, \mathrm{CDCl}_{3}, 298 \mathrm{~K}\right)$

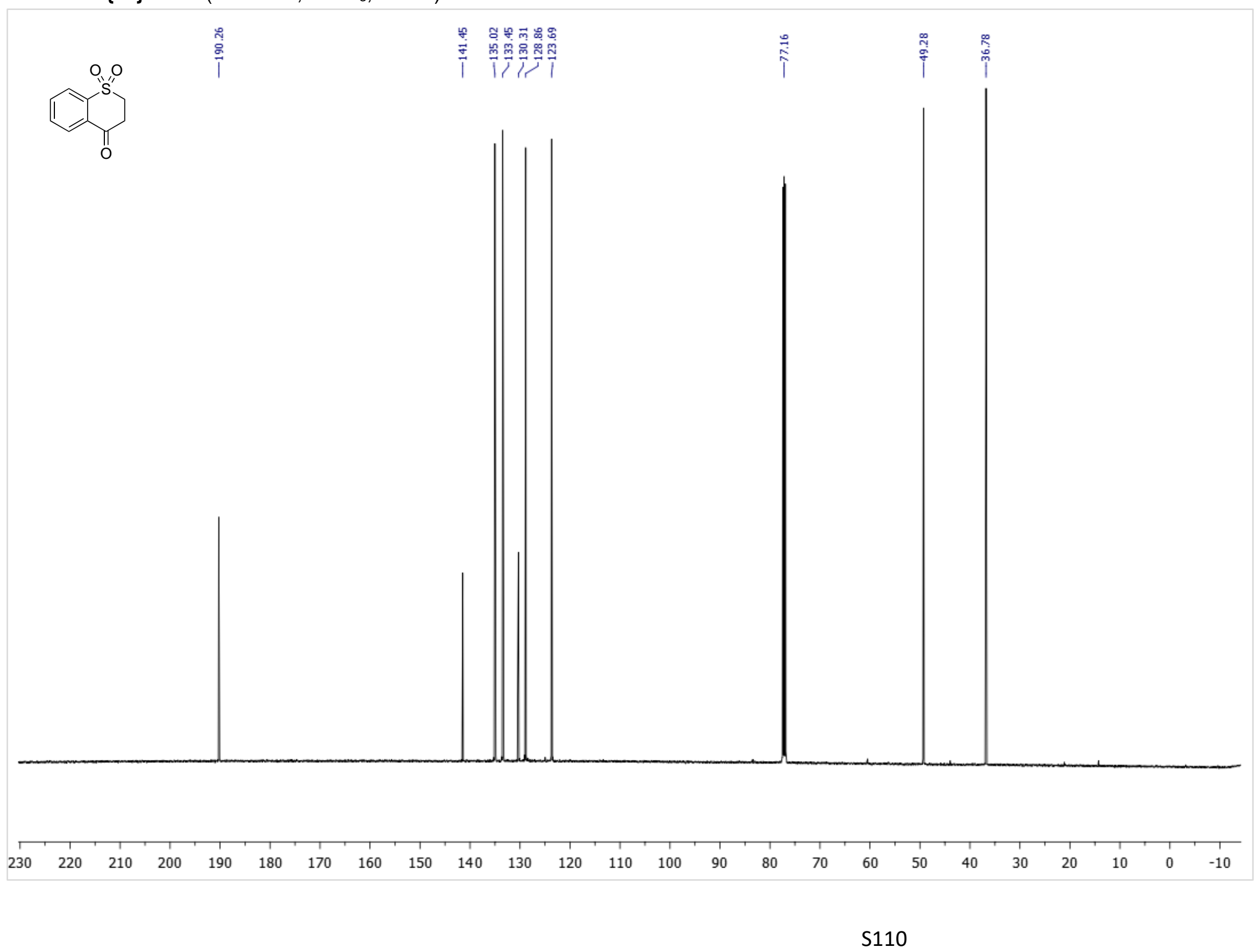


2-(methylsulfonyl)pyridine (3x)

${ }^{1} \mathbf{H}$ NMR $\left(500 \mathrm{MHz}, \mathrm{CDCl}_{3}, 298 \mathrm{~K}\right)$

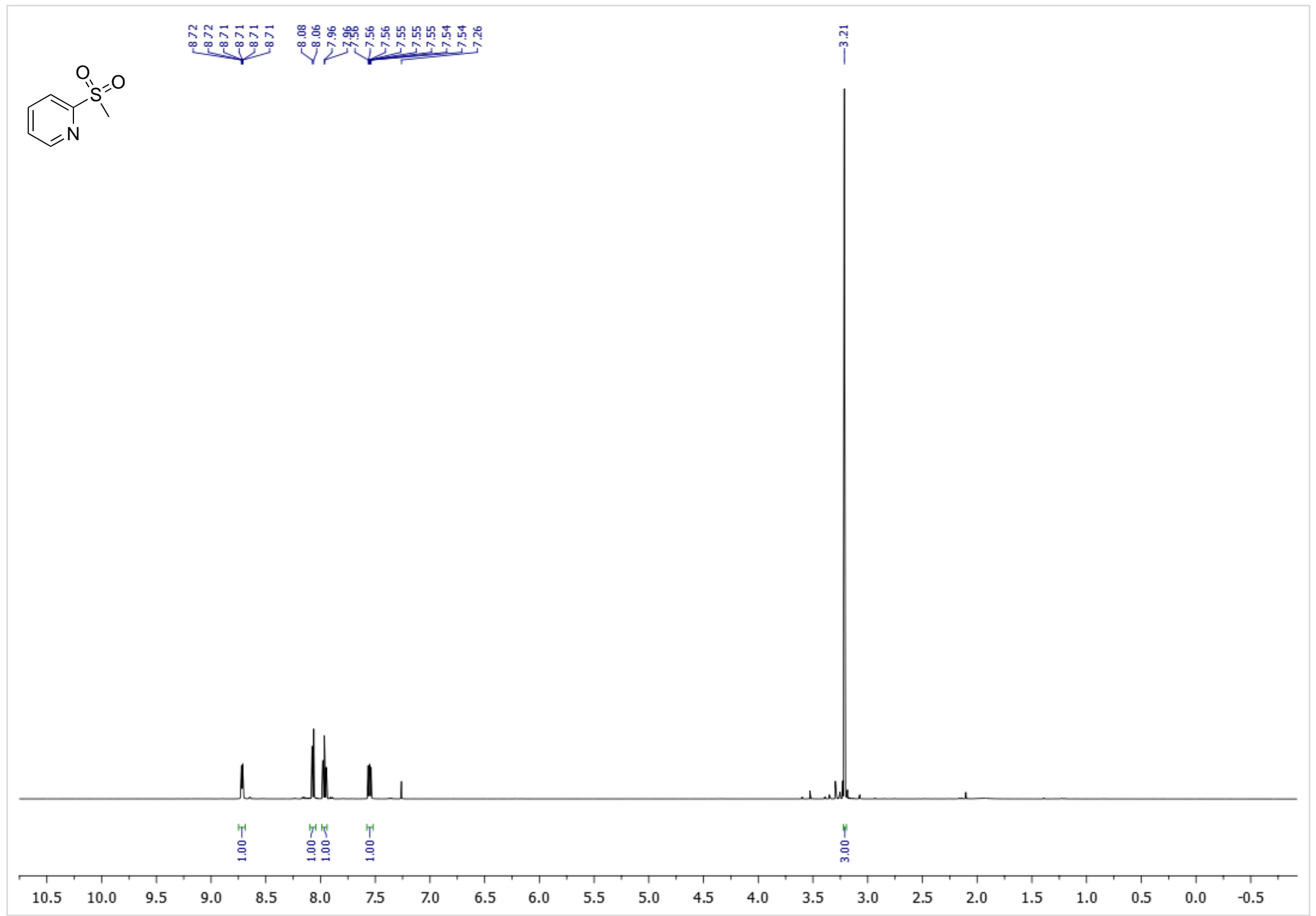

S111 
${ }^{13} \mathrm{C}\left\{{ }^{1} \mathrm{H}\right\}$ NMR $\left(101 \mathrm{MHz}, \mathrm{CDCl}_{3}, 298 \mathrm{~K}\right)$

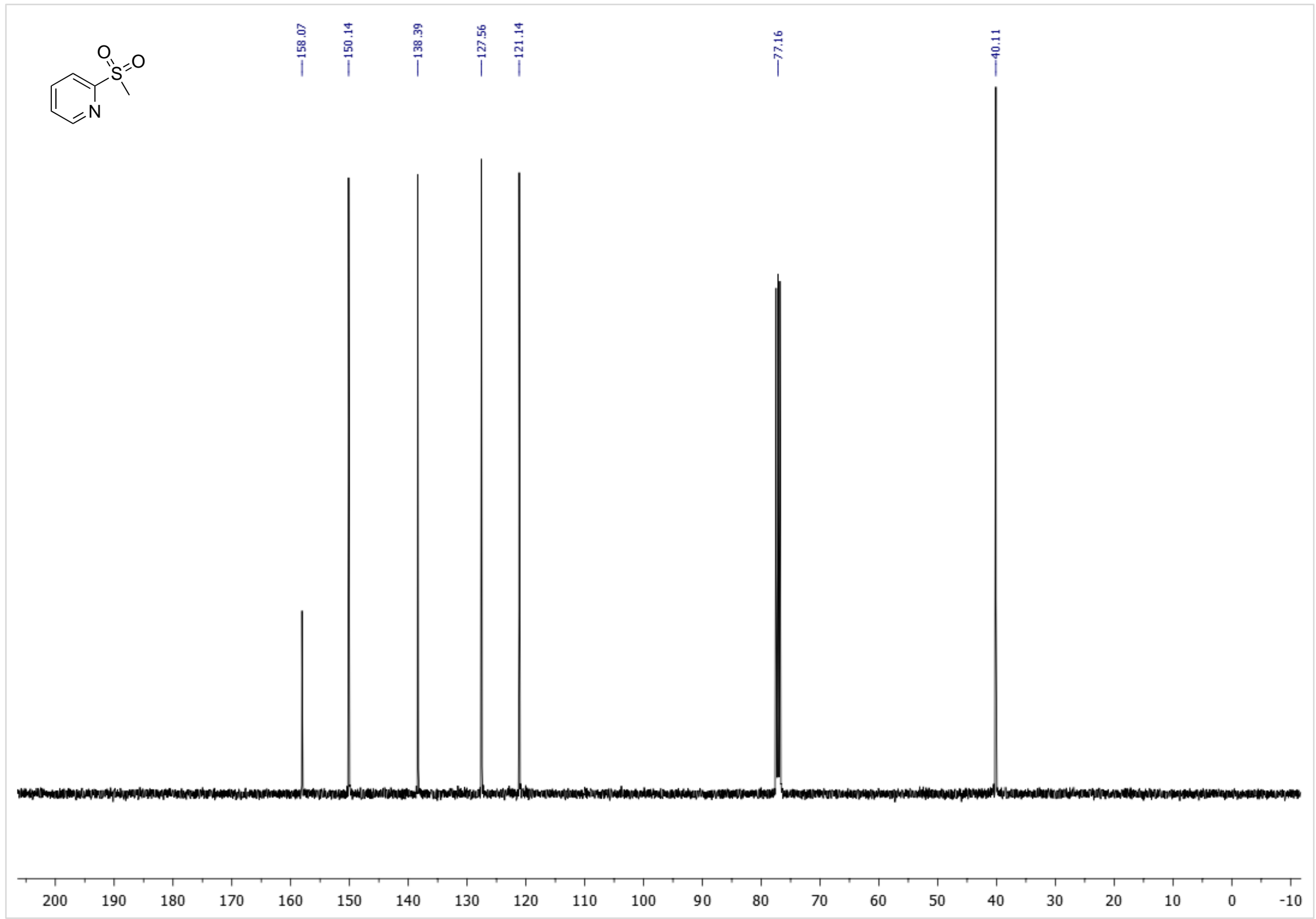

S112 
${ }^{1} \mathbf{H}$ NMR $\left(500 \mathrm{MHz}, \mathrm{CDCl}_{3}, 298 \mathrm{~K}\right)$

1-(butylsulfonyl)butane (3y)

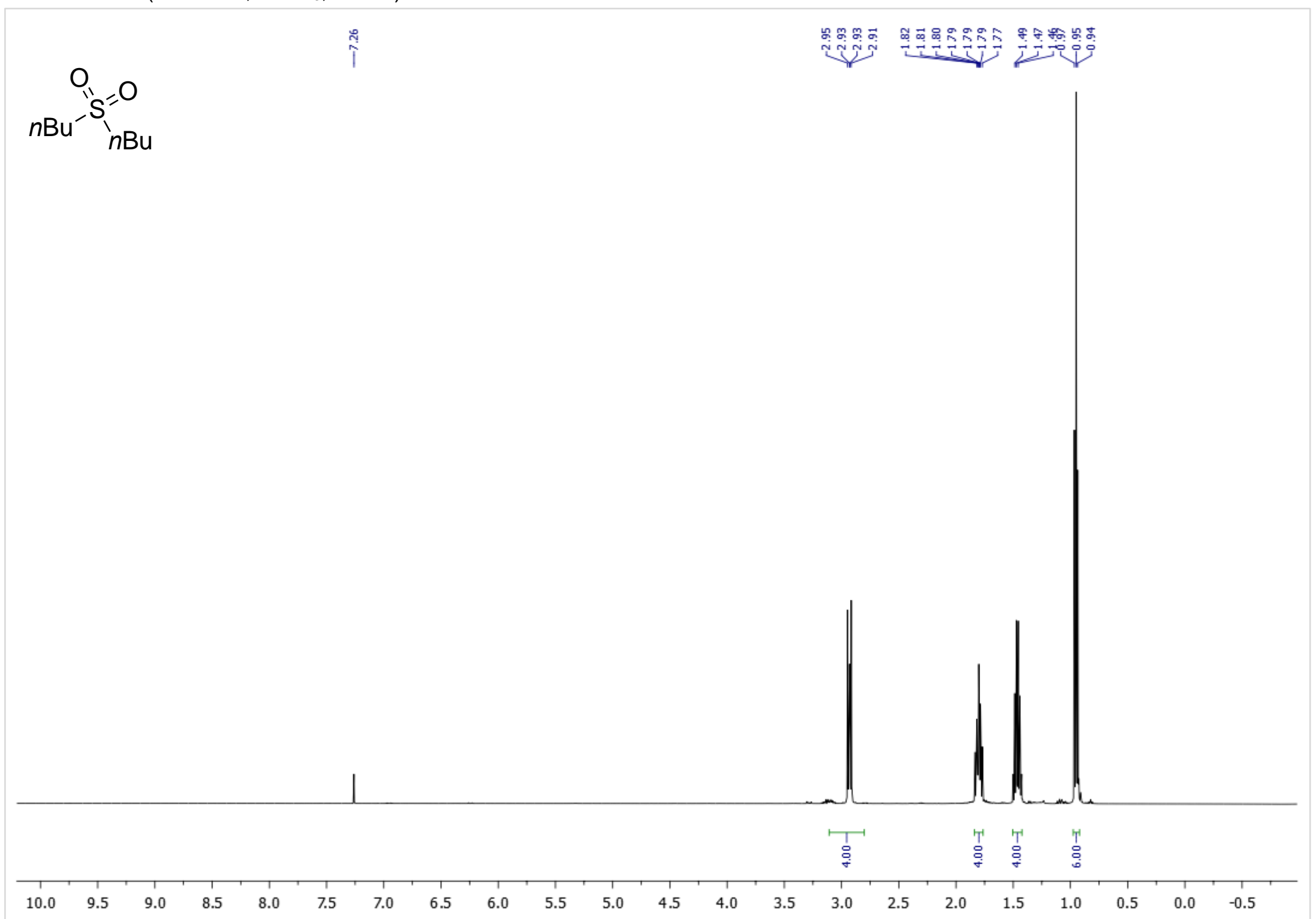

S113 
${ }^{13} \mathrm{C}\left\{{ }^{1} \mathrm{H}\right\}$ NMR $\left(126 \mathrm{MHz}, \mathrm{CDCl}_{3}, 298 \mathrm{~K}\right)$

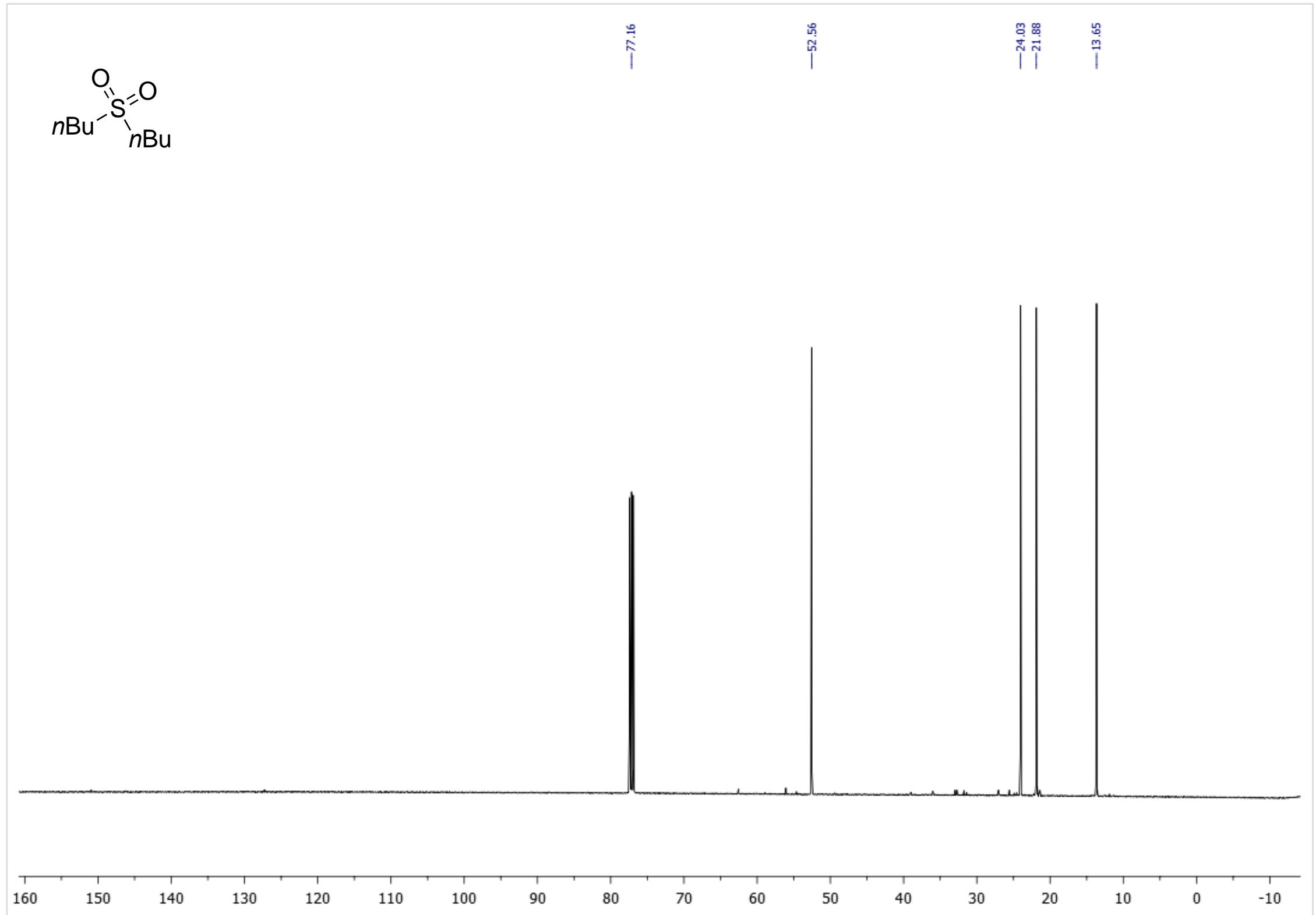


tetrahydro-2H-thiopyran 1,1-dioxide (3z)

${ }^{\mathbf{1}} \mathbf{H}$ NMR $\left(500 \mathrm{MHz}, \mathrm{CDCl}_{3}, 298 \mathrm{~K}\right)$

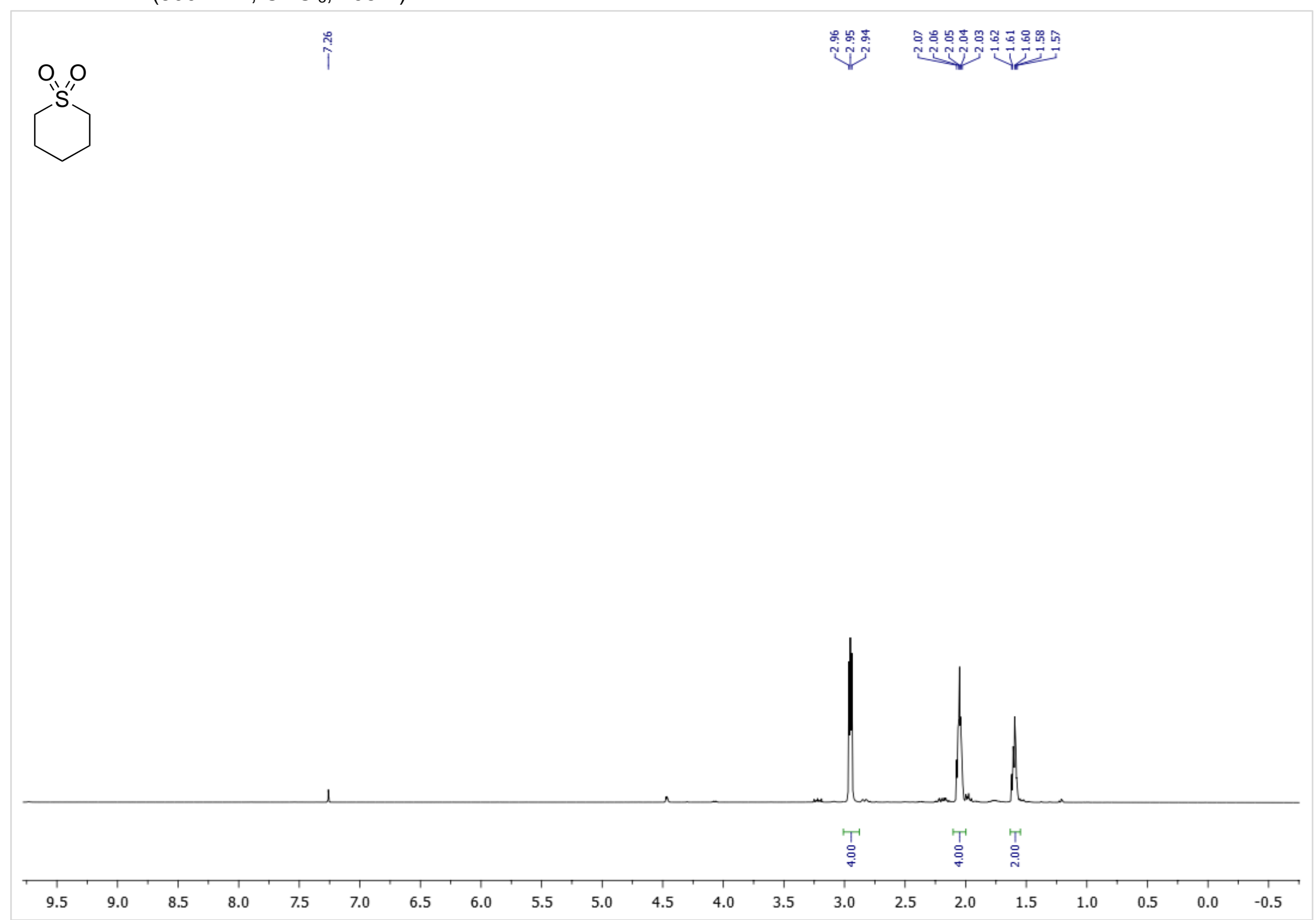


${ }^{13} \mathrm{C}\left\{{ }^{1} \mathrm{H}\right\}$ NMR $\left(101 \mathrm{MHz}, \mathrm{CDCl}_{3}, 298 \mathrm{~K}\right)$

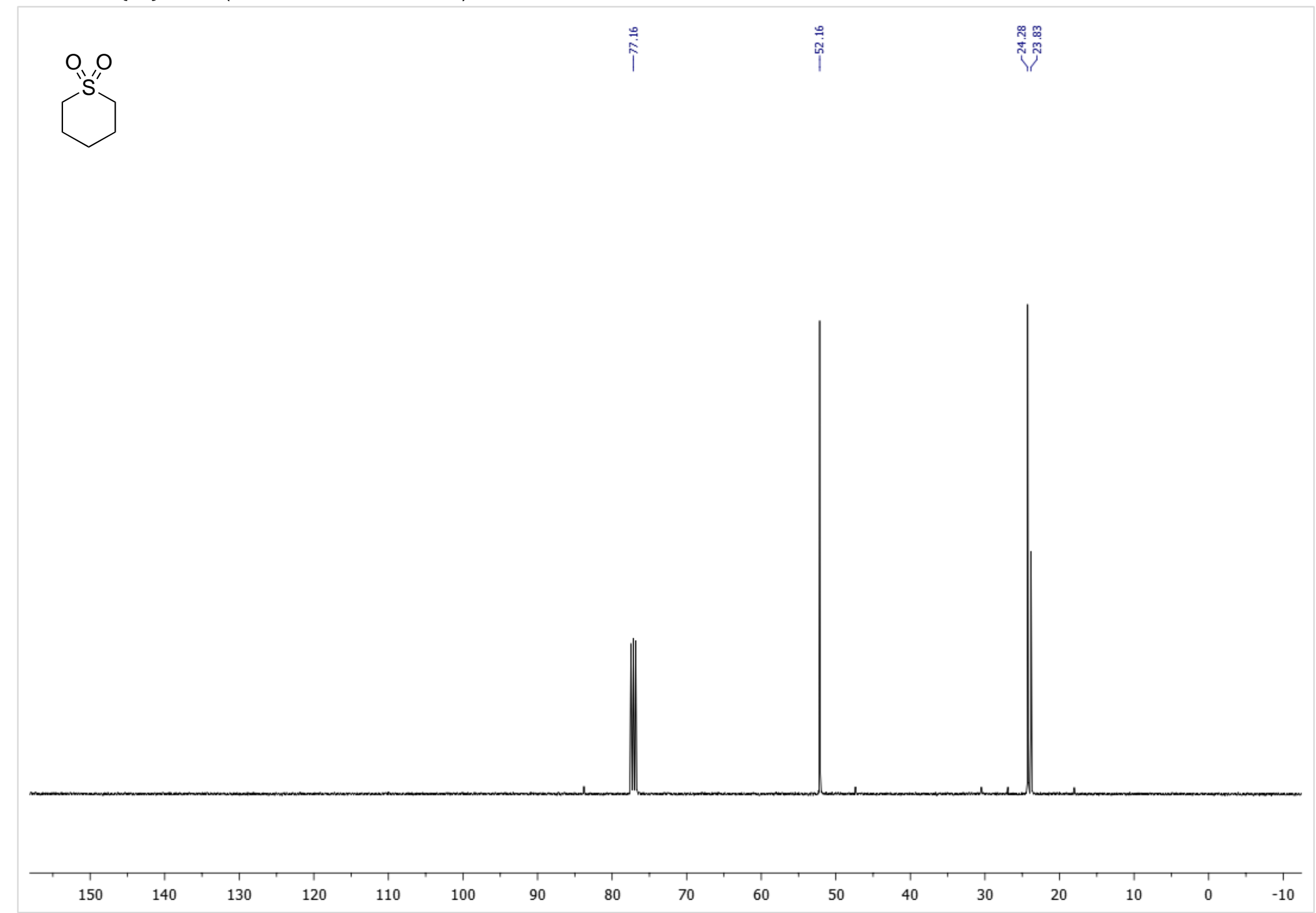


${ }^{1} \mathbf{H}$ NMR $\left(500 \mathrm{MHz}, \mathrm{CDCl}_{3}, 298 \mathrm{~K}\right)$

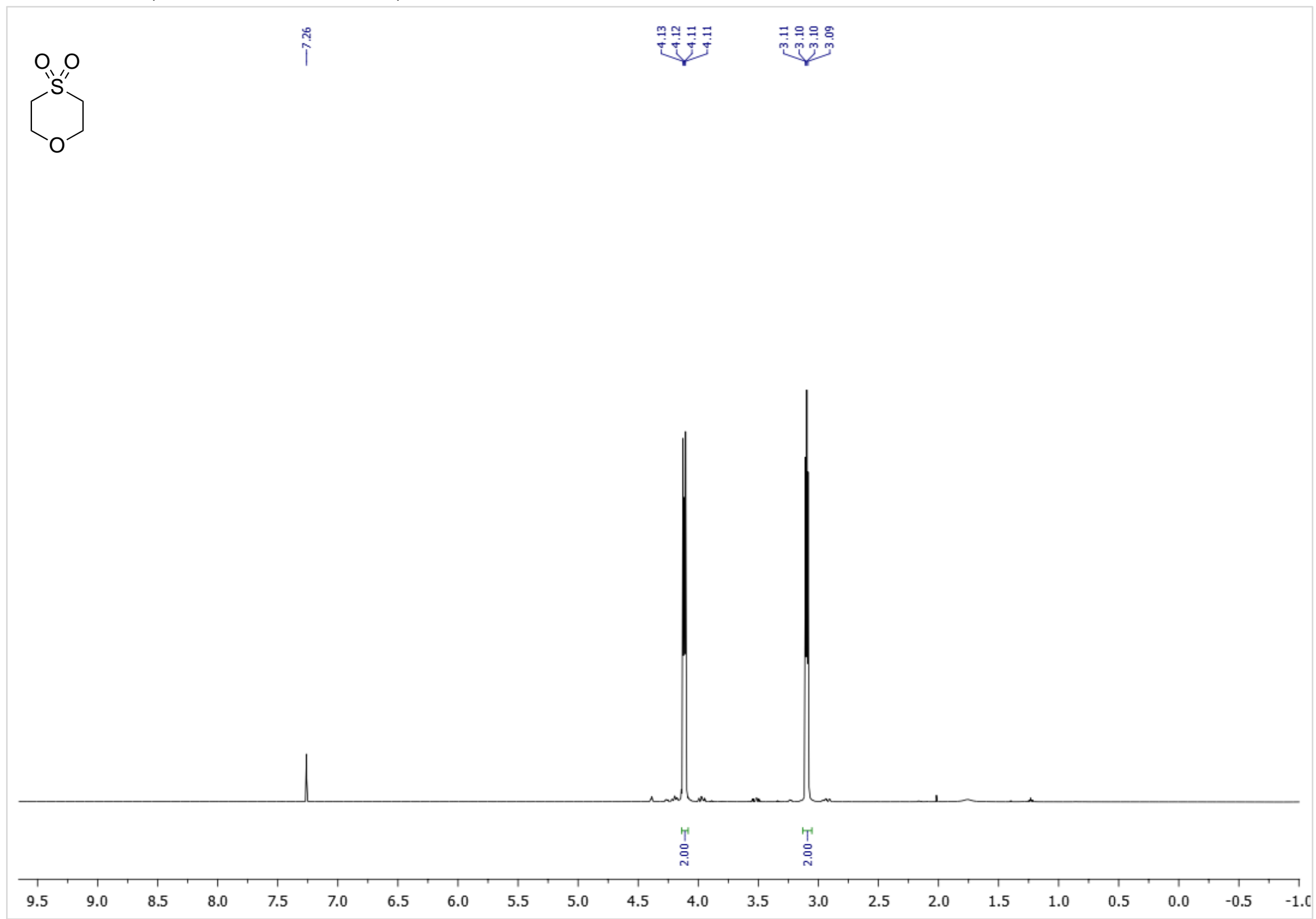


${ }^{13} \mathrm{C}\left\{{ }^{1} \mathrm{H}\right\}$ NMR $\left(101 \mathrm{MHz}, \mathrm{CDCl}_{3}, 298 \mathrm{~K}\right)$

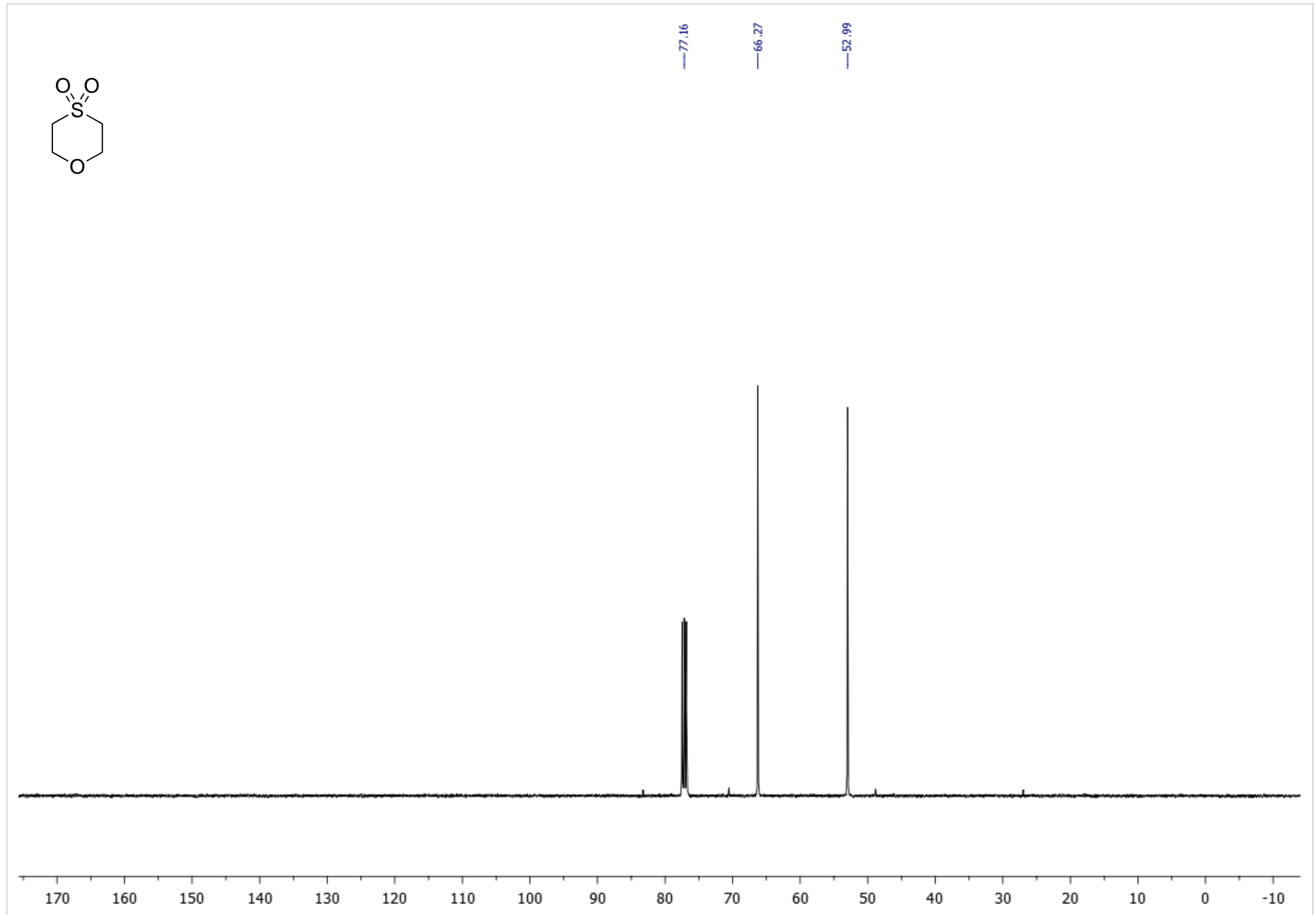


$N$-(methyl(oxo)(phenyl)- $\lambda^{6}$-sulfaneylidene)cyanamide (5a)

${ }^{1} \mathbf{H}$ NMR $\left(500 \mathrm{MHz}, \mathrm{CDCl}_{3}, 298 \mathrm{~K}\right)$

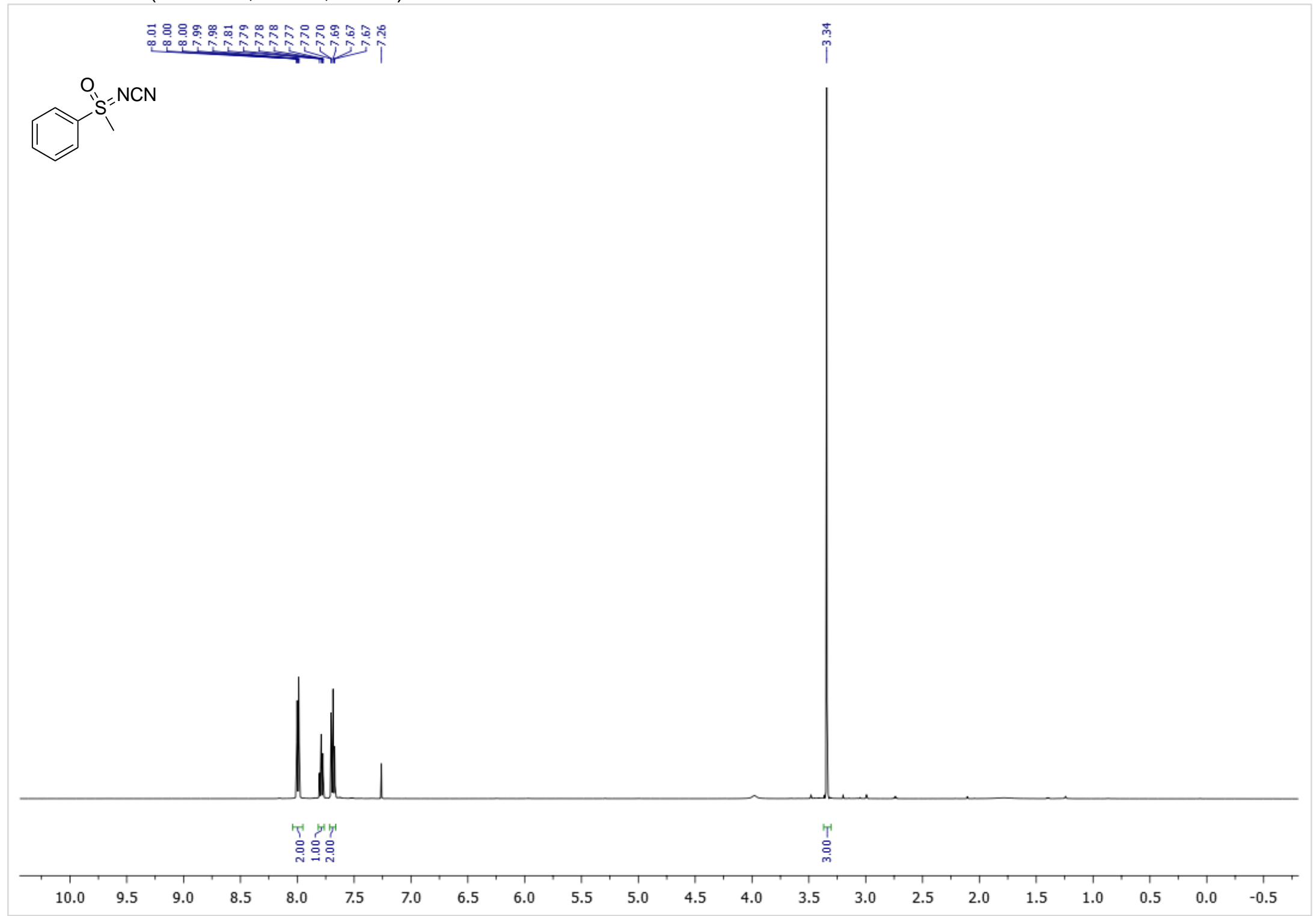


${ }^{13} \mathrm{C}\left\{{ }^{1} \mathrm{H}\right\}$ NMR $\left(126 \mathrm{MHz}, \mathrm{CDCl}_{3}, 298 \mathrm{~K}\right)$

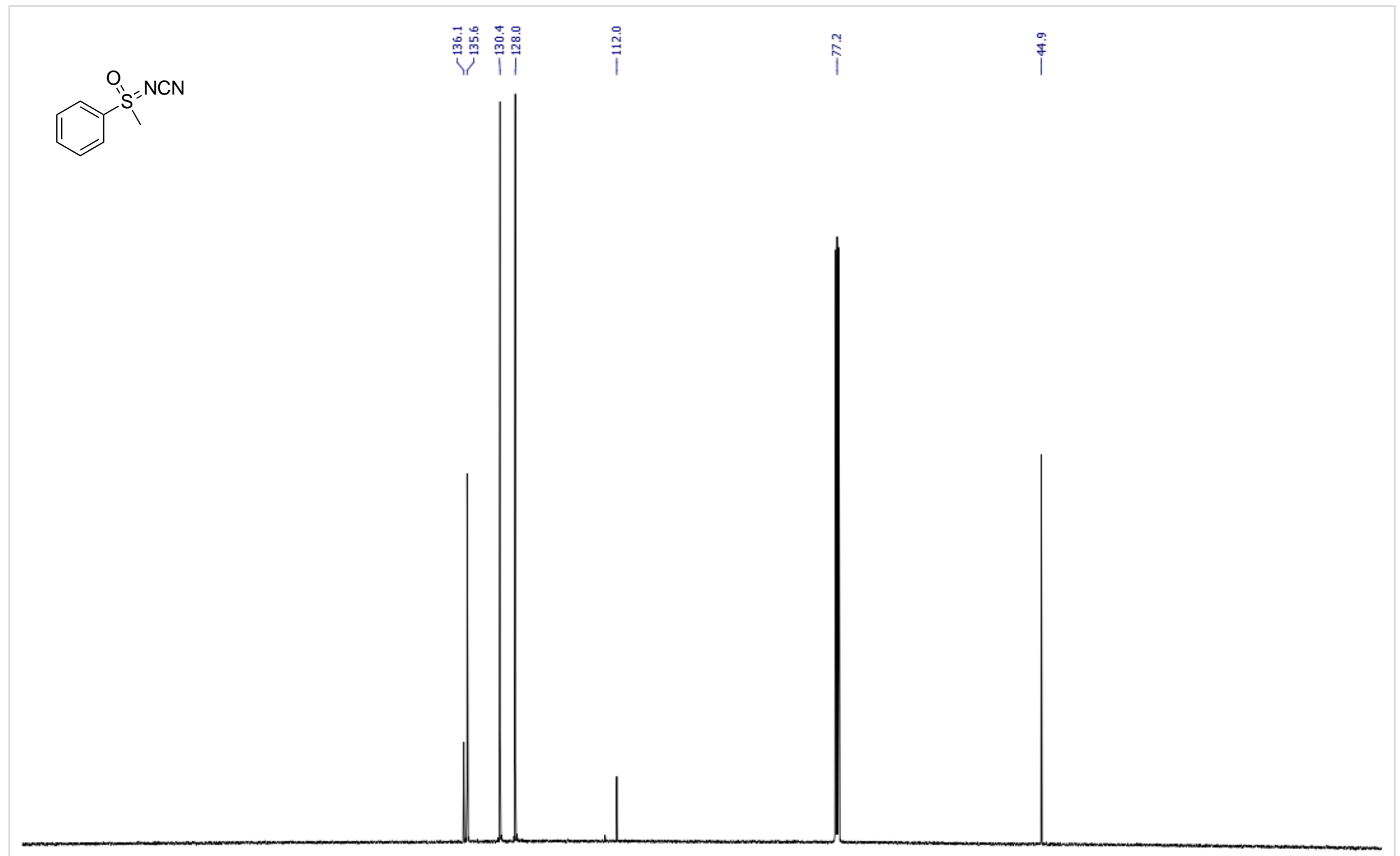

20

190

180

170 
$N$-(methyl(oxo)(p-tolyl)- $\lambda^{6}$-sulfaneylidene)cyanamide (5b)

${ }^{1} \mathrm{H}$ NMR $\left(500 \mathrm{MHz}, \mathrm{CDCl}_{3}, 298 \mathrm{~K}\right)$

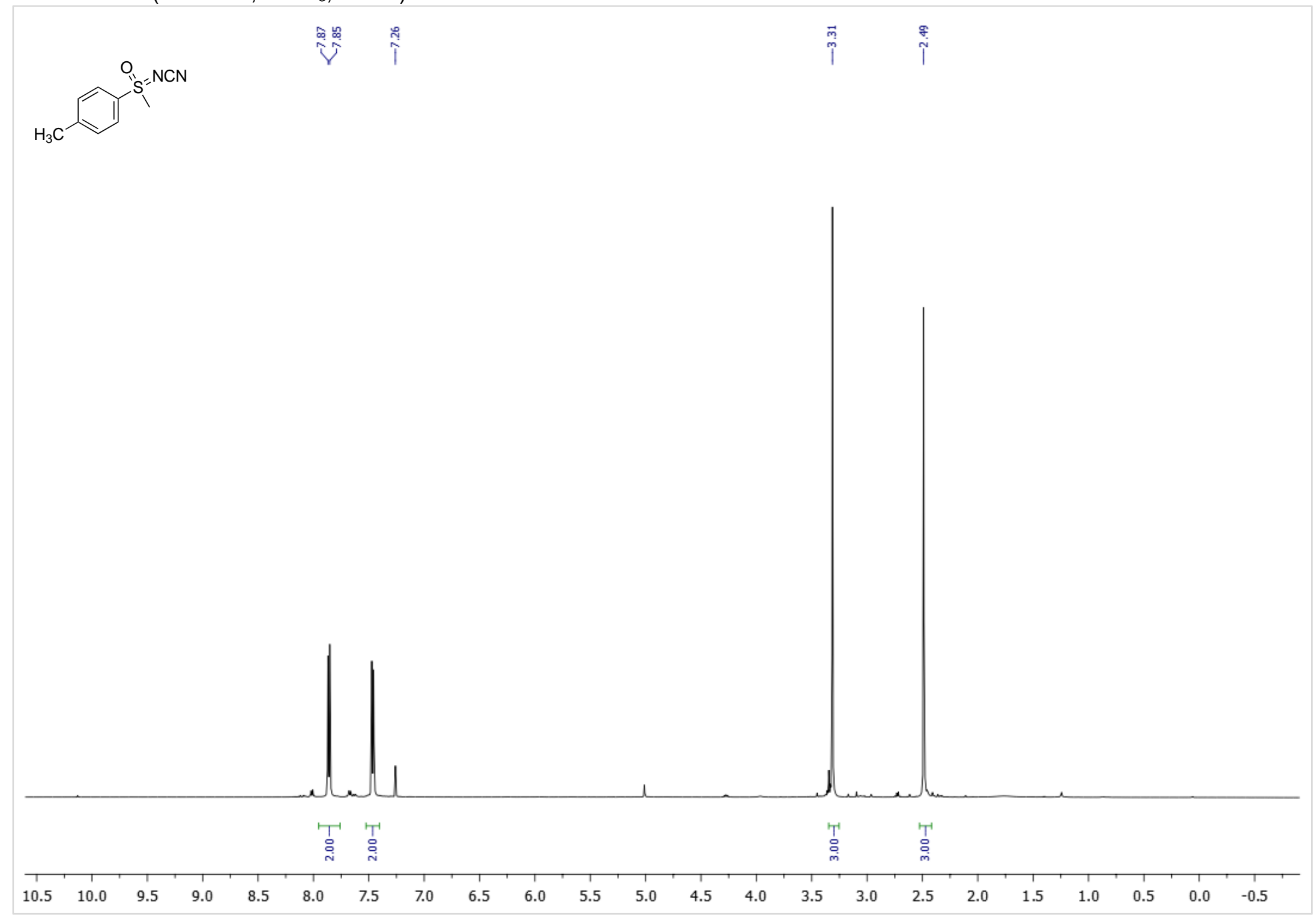


${ }^{13} \mathbf{C}\left\{{ }^{1} \mathrm{H}\right\}$ NMR $\left(126 \mathrm{MHz}, \mathrm{CDCl}_{3}, 298 \mathrm{~K}\right)$

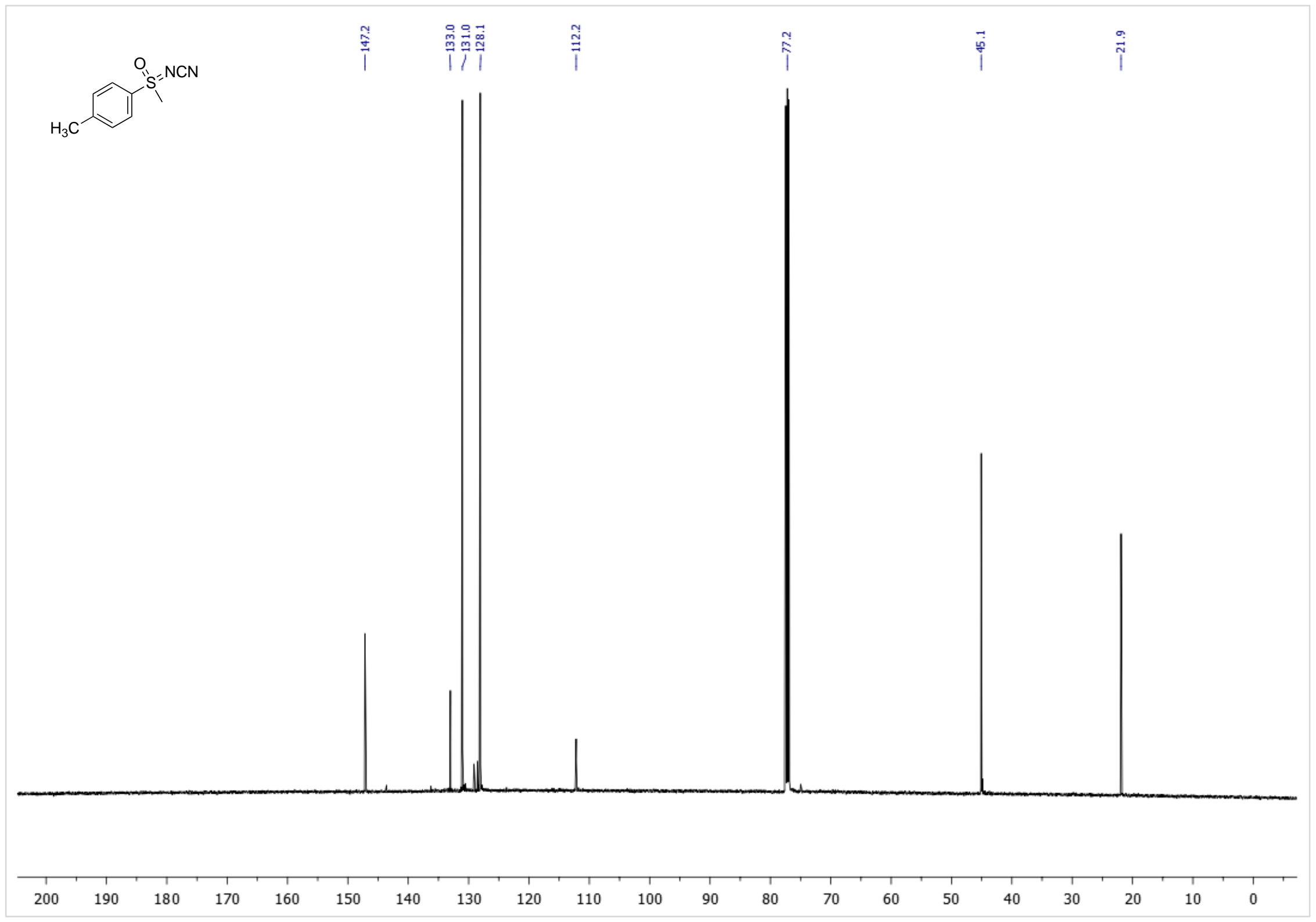

S122 
$N$-((4-bromophenyl)(methyl)(oxo)- $\lambda^{6}$-sulfaneylidene)cyanamide (5c)

${ }^{1} \mathrm{H}$ NMR $\left(500 \mathrm{MHz}, \mathrm{CDCl}_{3}, 298 \mathrm{~K}\right)$

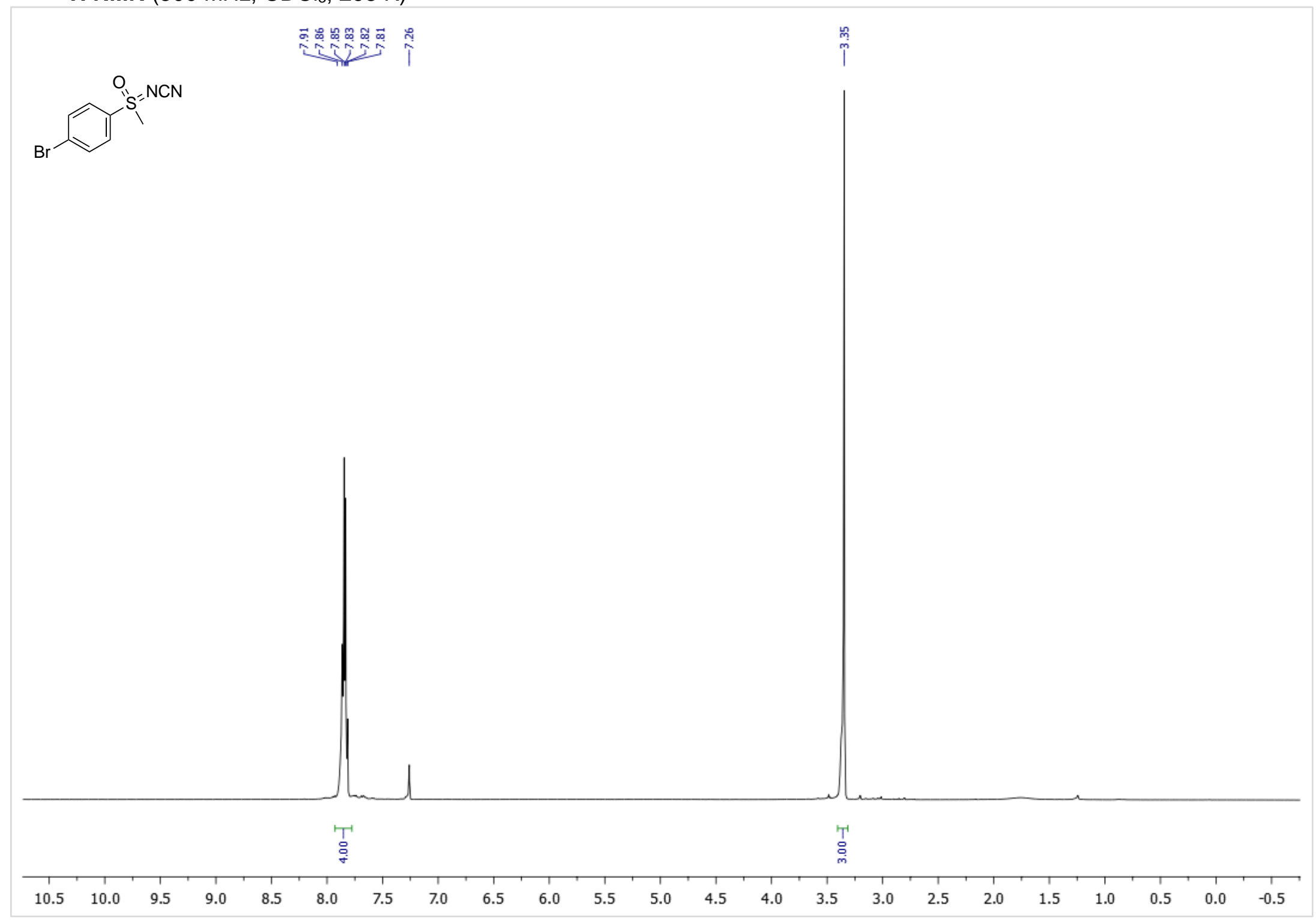


${ }^{13} \mathrm{C}\left\{{ }^{1} \mathrm{H}\right\}$ NMR $\left(126 \mathrm{MHz}, \mathrm{CDCl}_{3}, 298 \mathrm{~K}\right)$

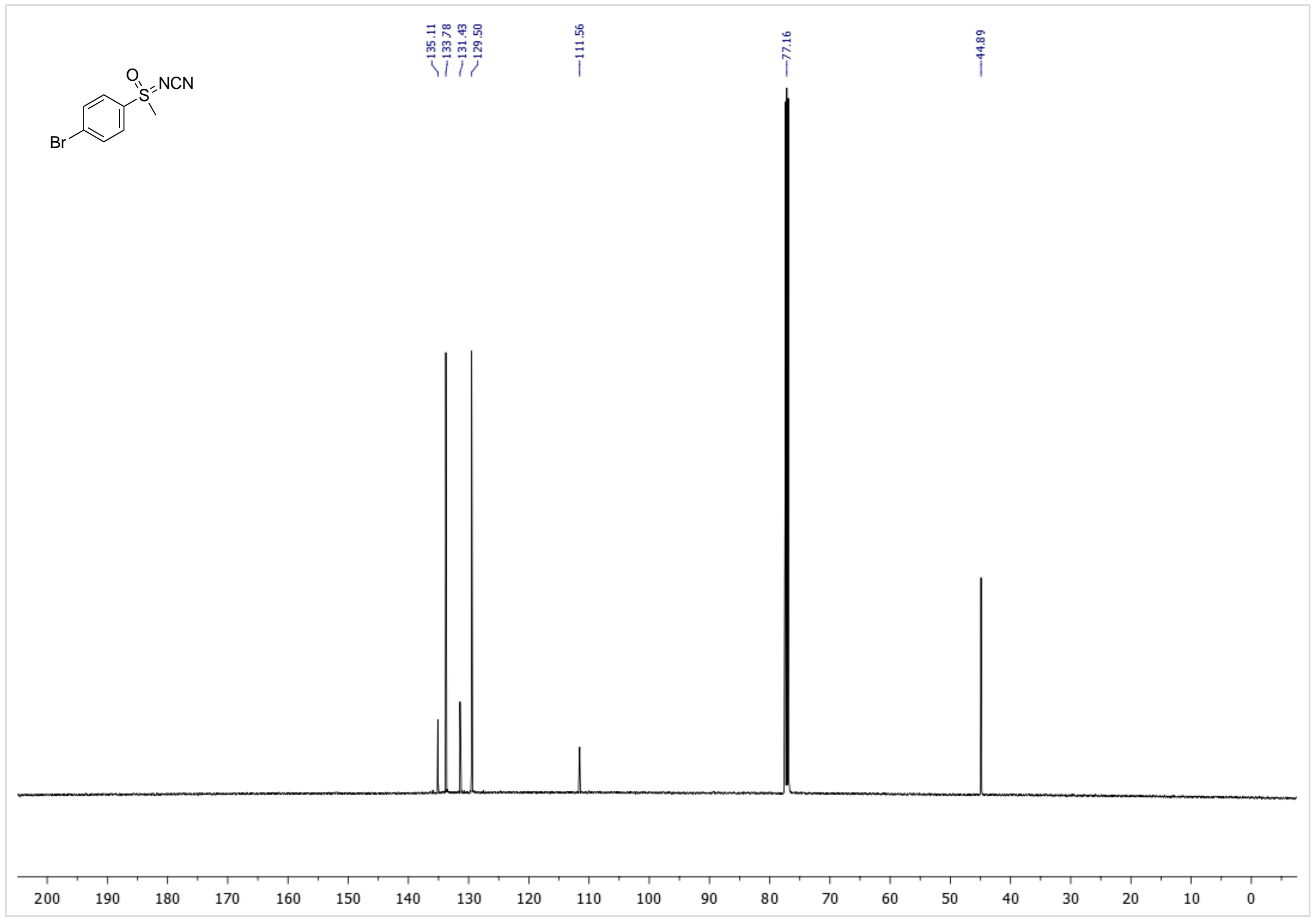

S124 
$N$-((4-chlorophenyl)(methyl)(oxo)- $\lambda^{6}$-sulfaneylidene)cyanamide (5d)

${ }^{1} \mathrm{H}$ NMR $\left(500 \mathrm{MHz}, \mathrm{CDCl}_{3}, 298 \mathrm{~K}\right)$

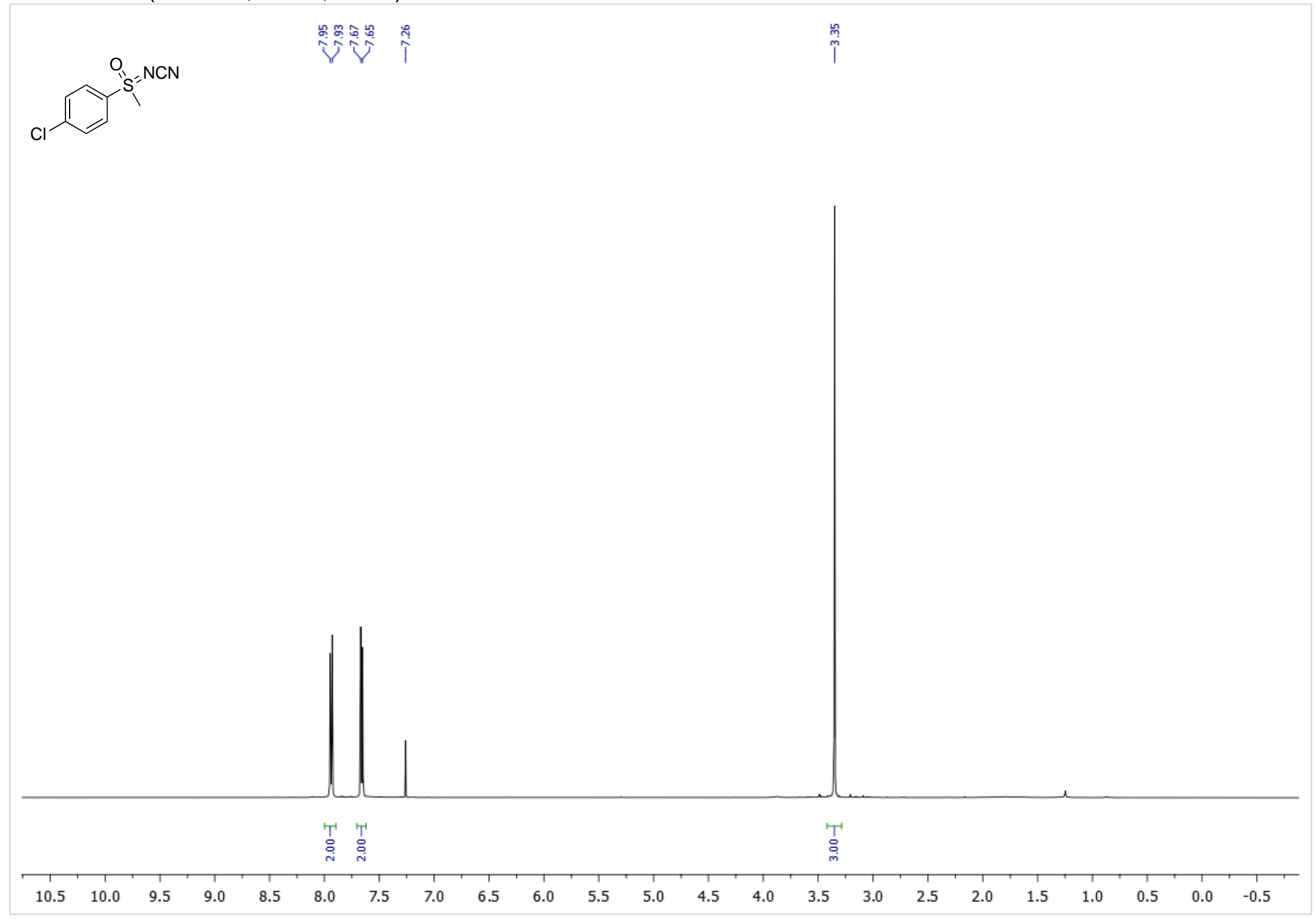


${ }^{13} \mathbf{C}\left\{{ }^{1} \mathrm{H}\right\}$ NMR $\left(126 \mathrm{MHz}, \mathrm{CDCl}_{3}, 298 \mathrm{~K}\right)$

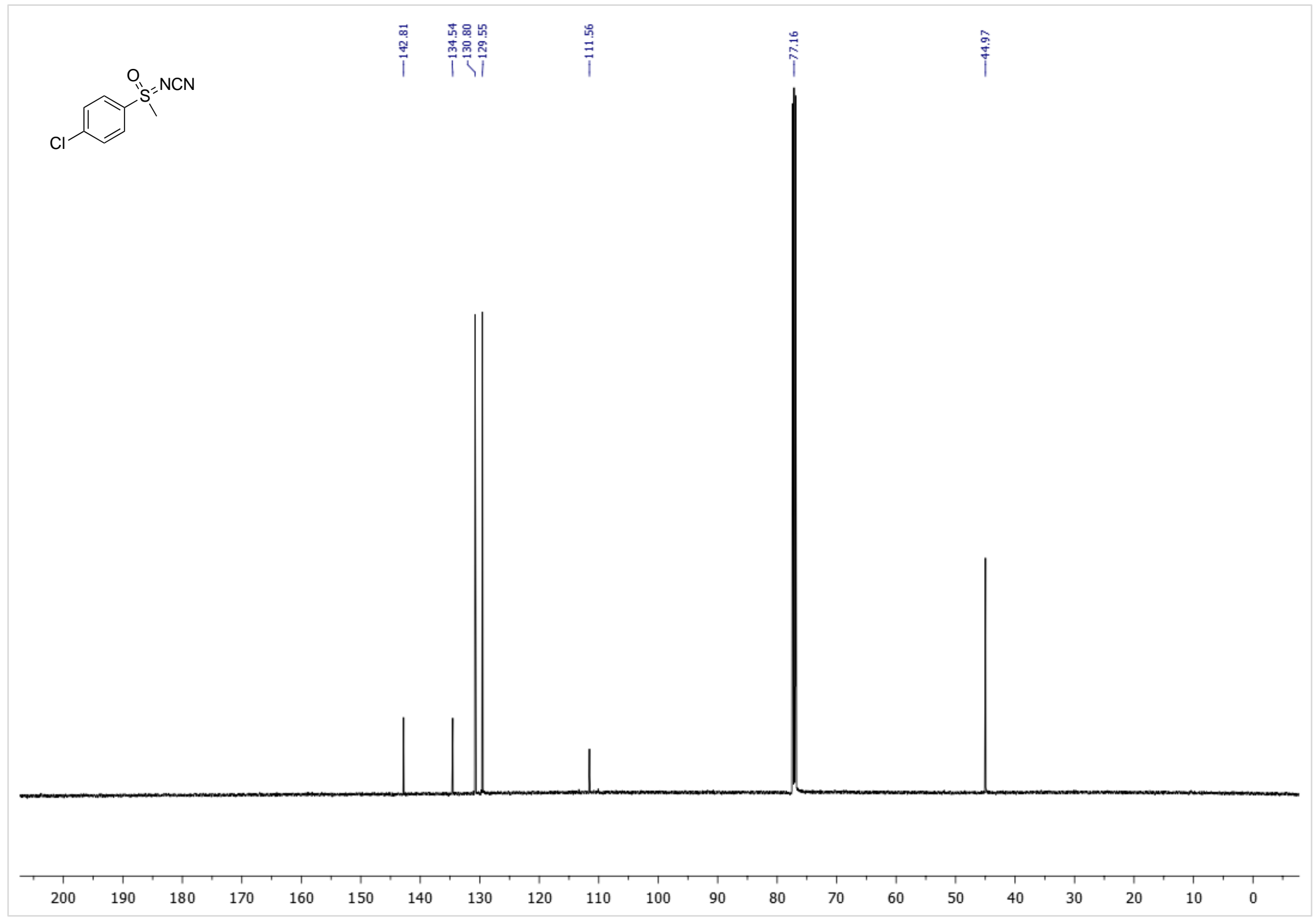

S126 
$N$-((4-fluorophenyl)(methyl)(oxo)- $\lambda^{6}$-sulfaneylidene)cyanamide (5e)

${ }^{1} \mathrm{H}$ NMR $\left(500 \mathrm{MHz}, \mathrm{CDCl}_{3}, 298 \mathrm{~K}\right)$

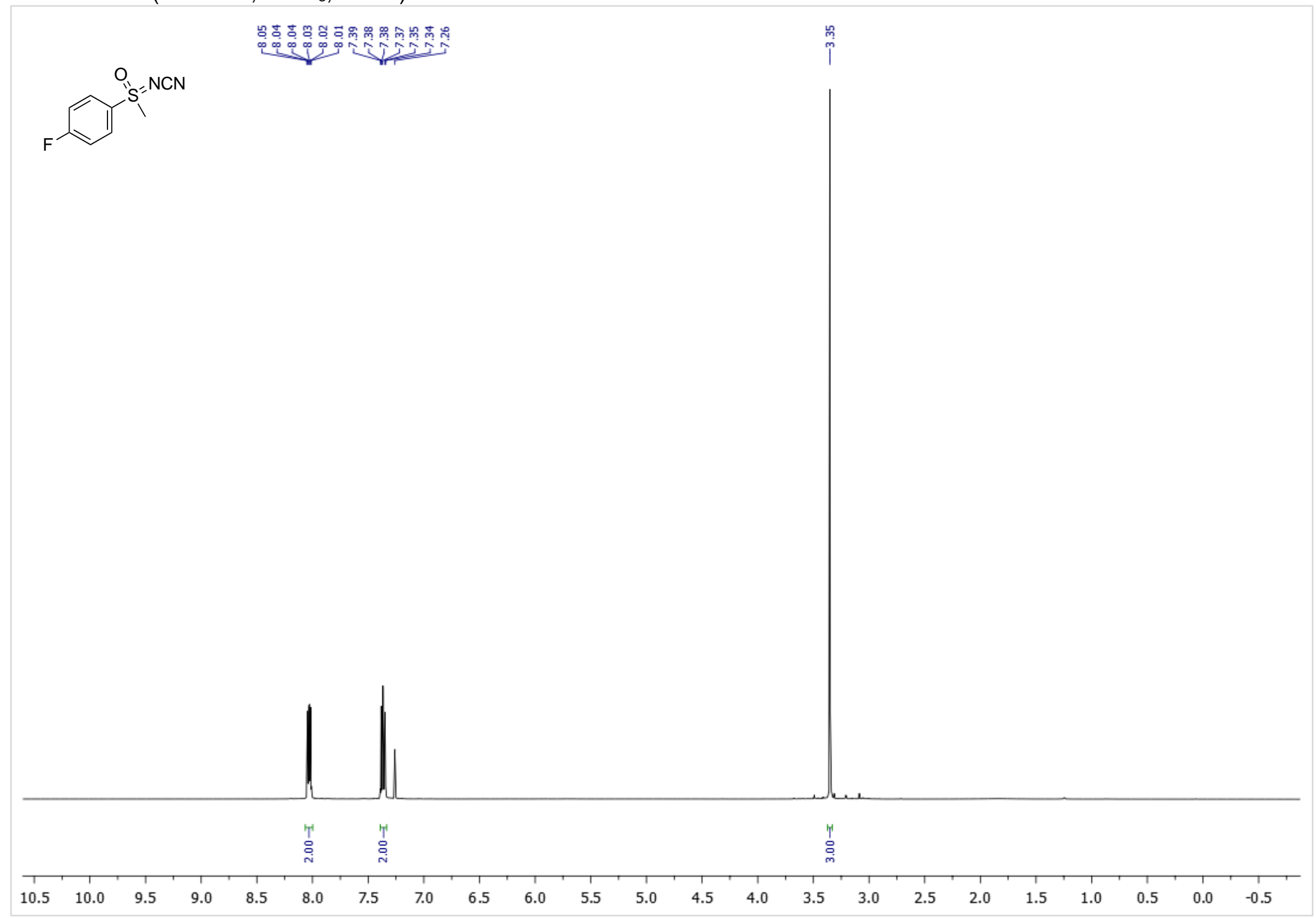


${ }^{13} \mathrm{C}\left\{{ }^{1} \mathrm{H}\right\}$ NMR $\left(126 \mathrm{MHz}, \mathrm{CDCl}_{3}, 298 \mathrm{~K}\right)$

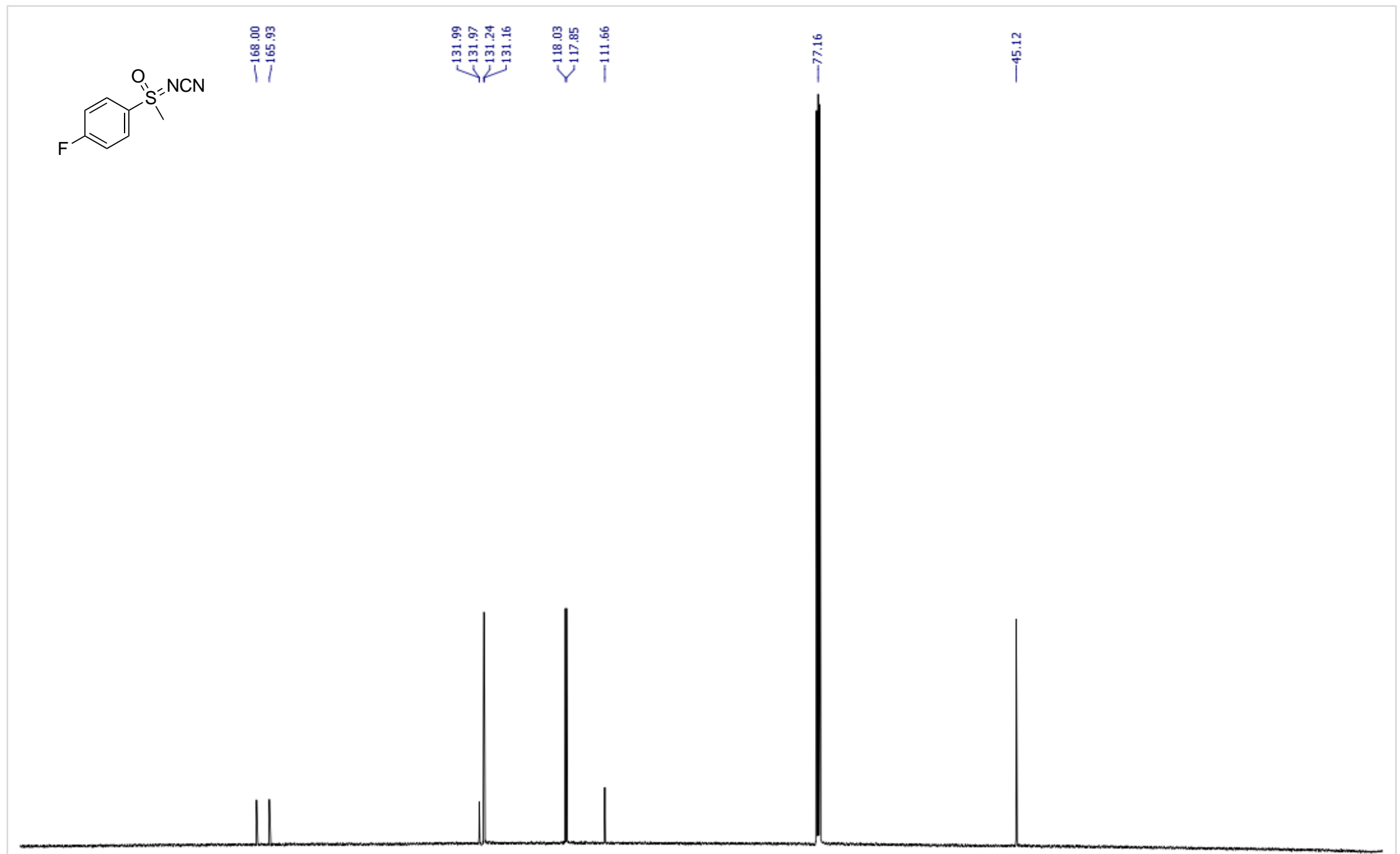


${ }^{19} \mathrm{~F}\left\{{ }^{1} \mathrm{H}\right\} \operatorname{NMR}\left(471 \mathrm{MHz}, \mathrm{CDCl}_{3}, 298 \mathrm{~K}\right)$

\section{i.}

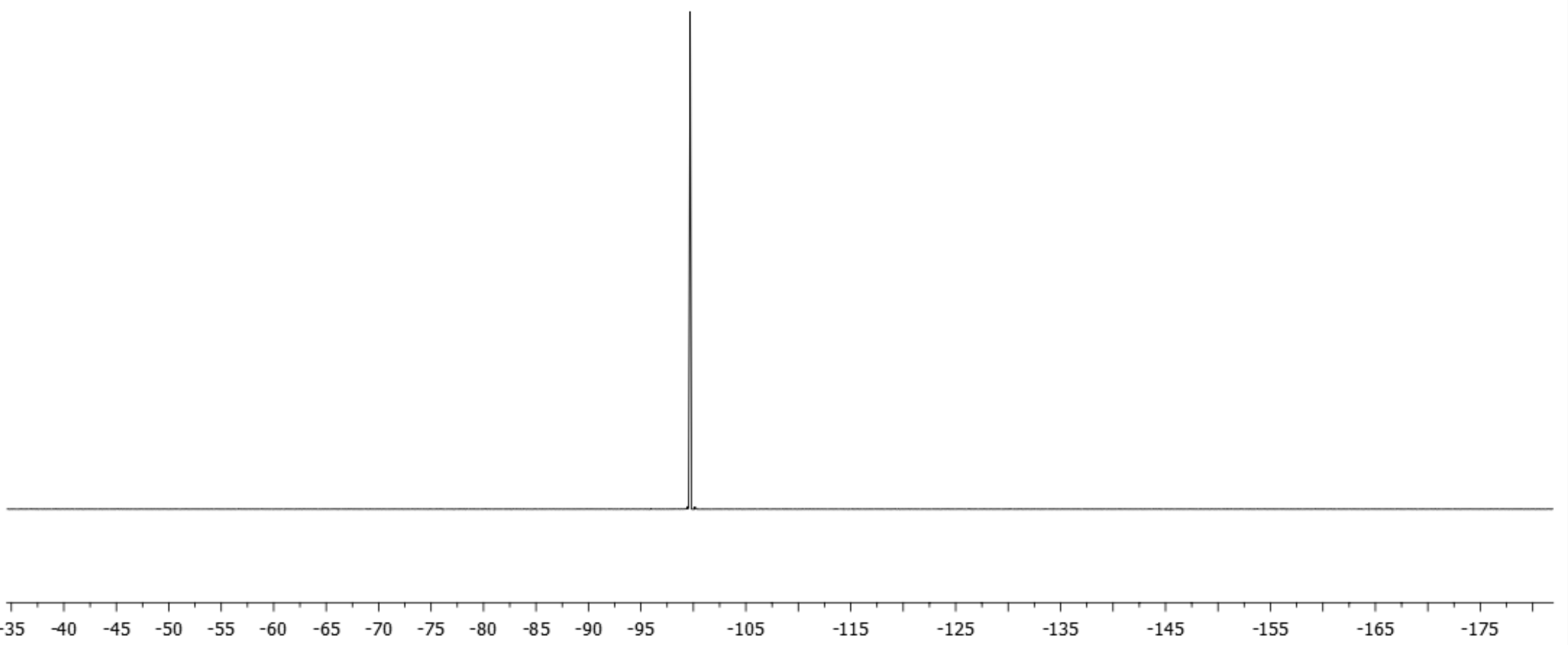


$N$-((4-cyanophenyl)(methyl)(oxo)- $\lambda^{6}$-sulfaneylidene)cyanamide (5f)

${ }^{1} \mathrm{H}$ NMR $\left(500 \mathrm{MHz}, \mathrm{CDCl}_{3}, 298 \mathrm{~K}\right)$

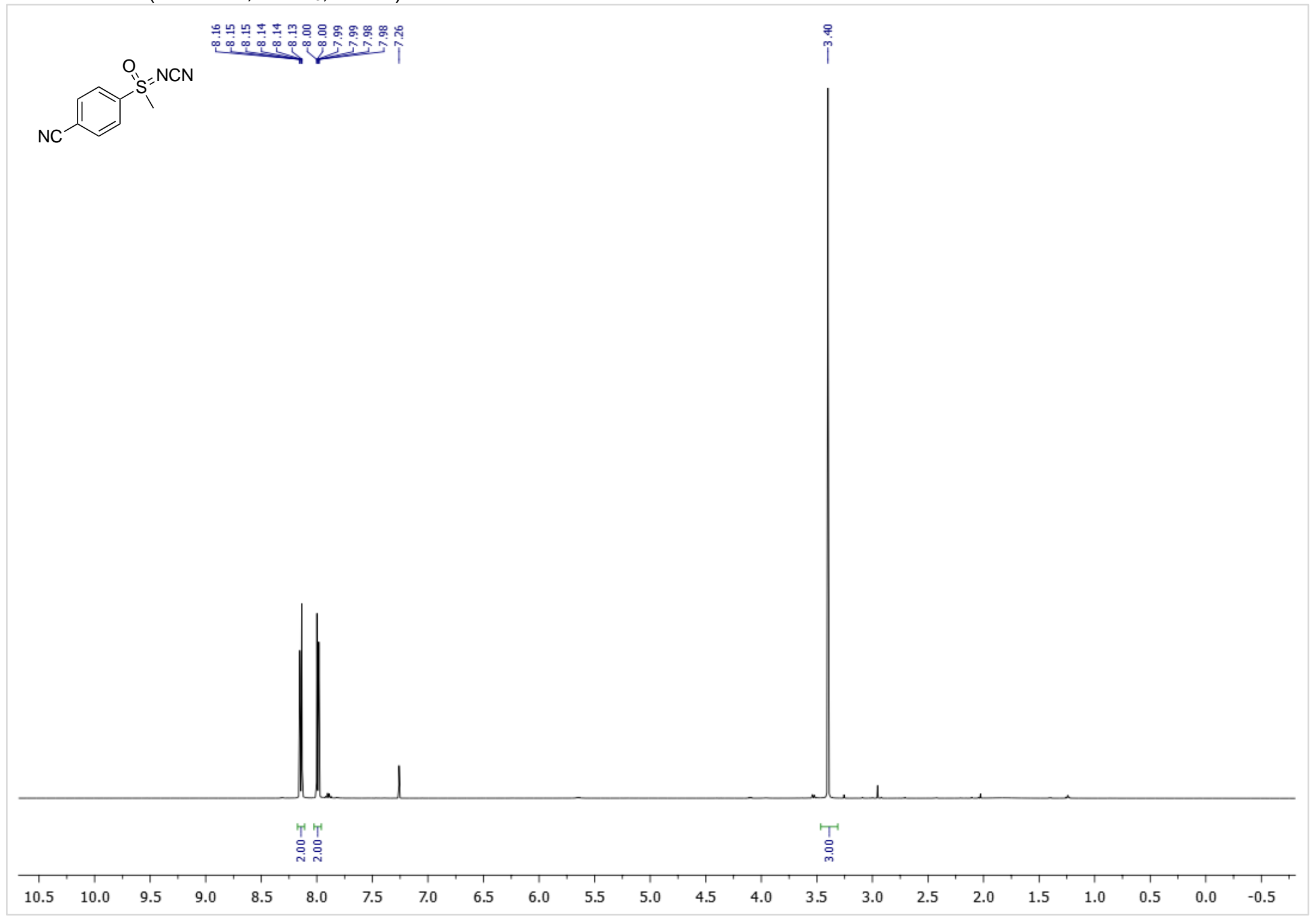


${ }^{13} \mathrm{C}\left\{{ }^{1} \mathrm{H}\right\}$ NMR $\left(126 \mathrm{MHz}, \mathrm{CDCl}_{3}, 298 \mathrm{~K}\right)$

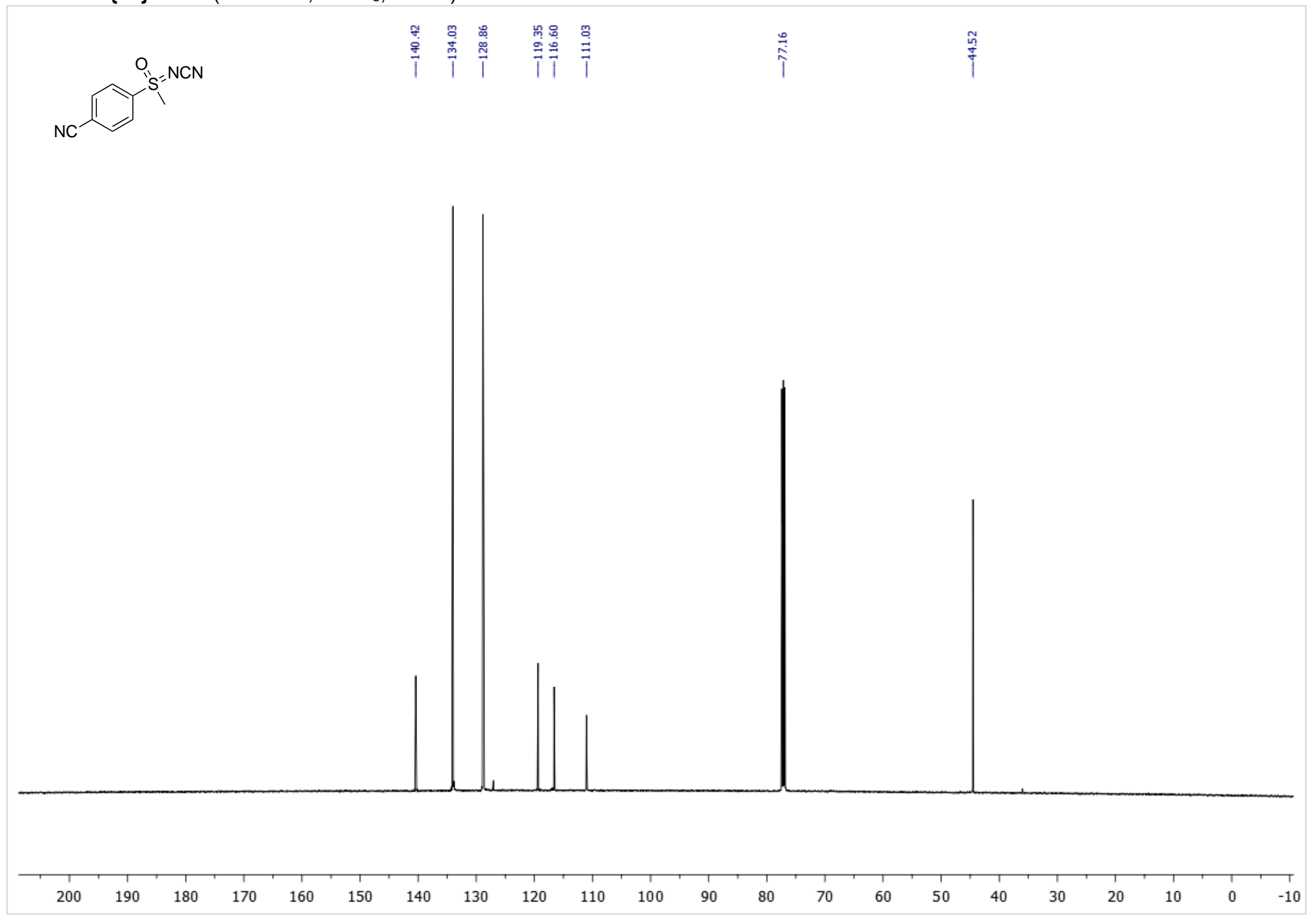

S131 
$N$-((4-methoxyphenyl)(methyl)(oxo)- $\lambda^{6}$-sulfaneylidene)cyanamide (5g)

${ }^{1} \mathrm{H}$ NMR $\left(500 \mathrm{MHz}, \mathrm{CDCl}_{3}, 298 \mathrm{~K}\right)$

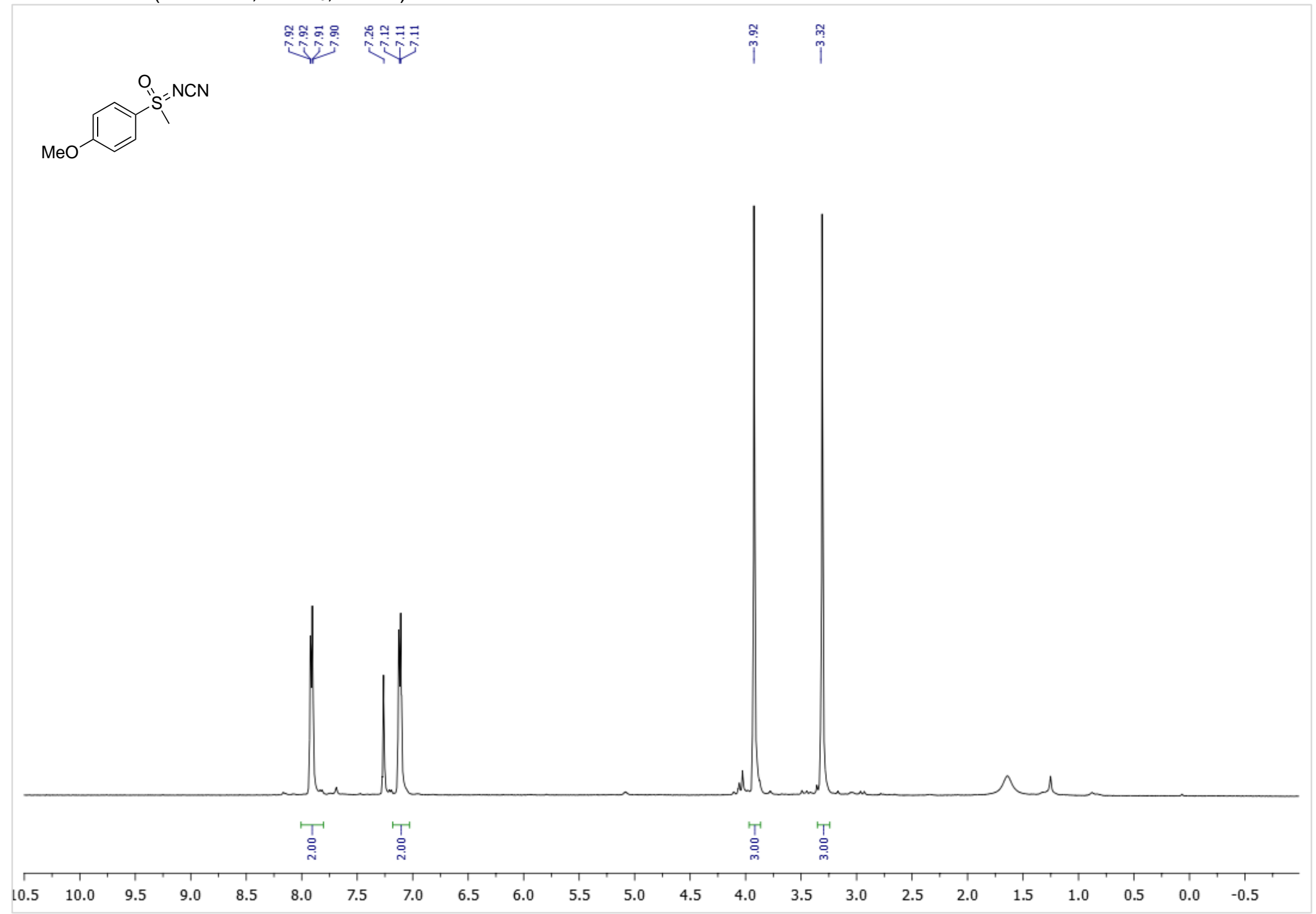


${ }^{13} \mathrm{C}\left\{{ }^{1} \mathrm{H}\right\}$ NMR $\left(126 \mathrm{MHz}, \mathrm{CDCl}_{3}, 298 \mathrm{~K}\right)$

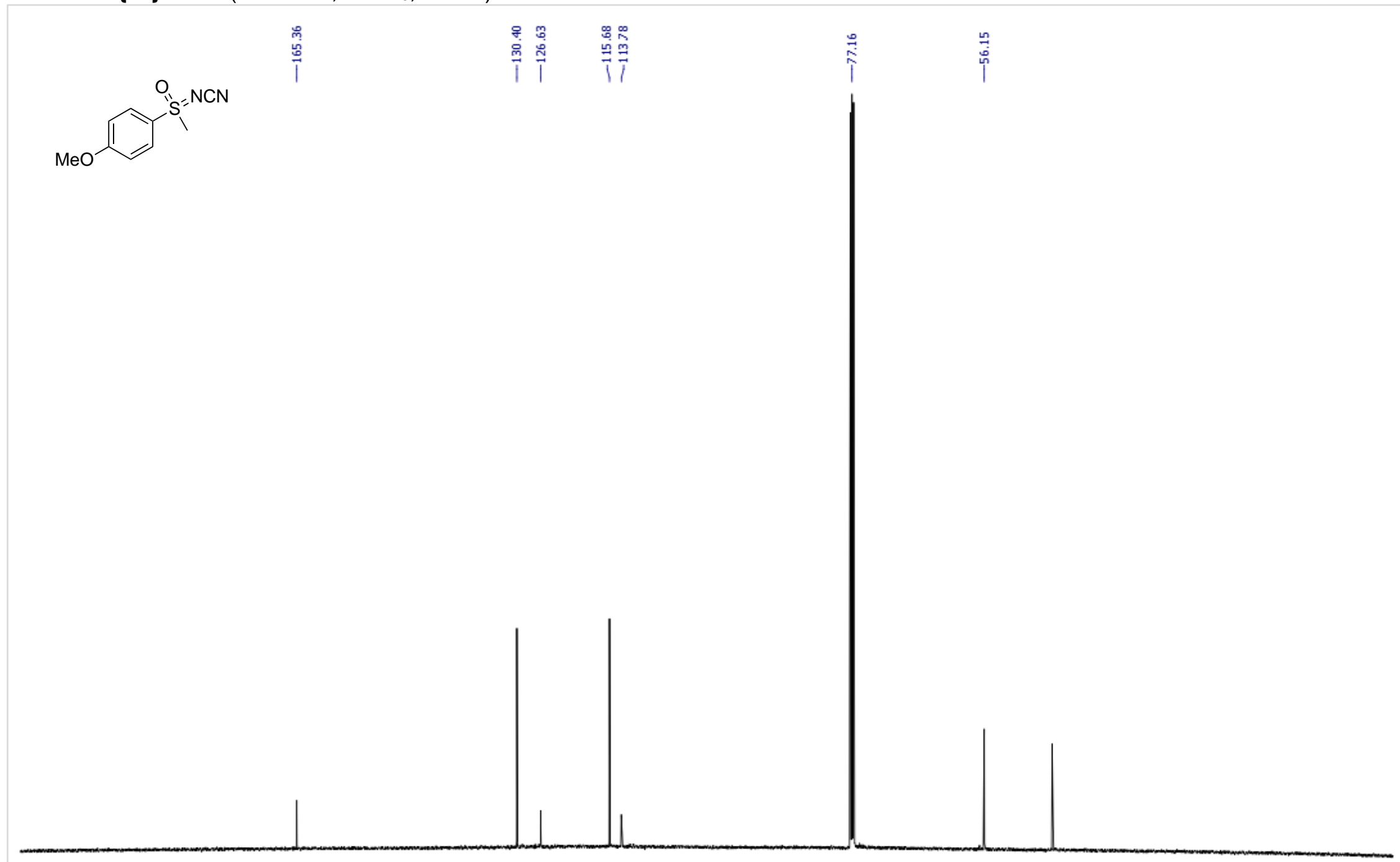

200

$\begin{array}{llll}190 & 180 & 170 & 160\end{array}$

$150 \quad 140$

$130 \quad 120$

$10 \quad 100$ 
$N$-((3-bromophenyl)(methyl)(oxo)- $\lambda^{6}$-sulfaneylidene)cyanamide (5h)

${ }^{1} \mathrm{H}$ NMR $\left(500 \mathrm{MHz}, \mathrm{CDCl}_{3}, 298 \mathrm{~K}\right)$

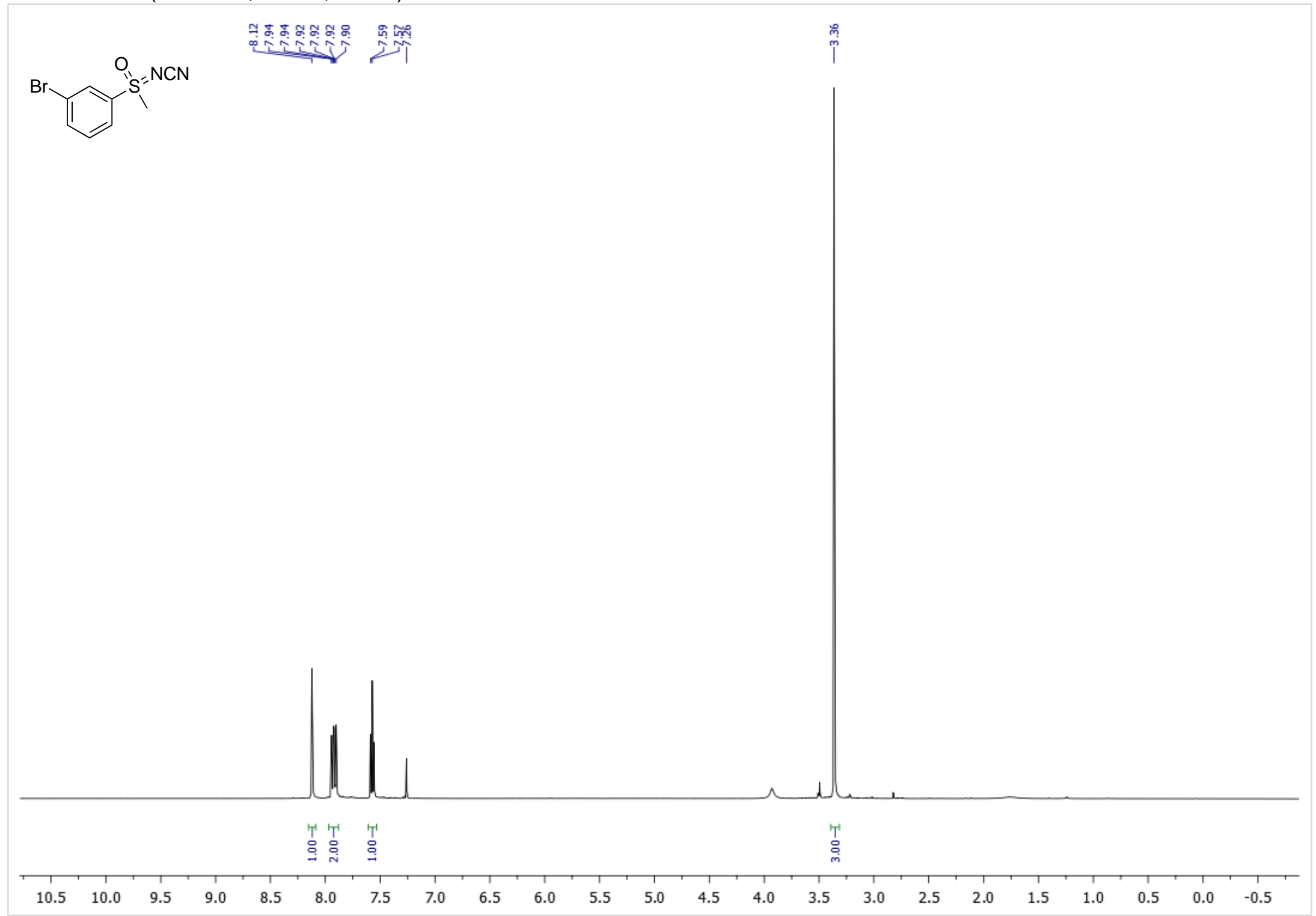


${ }^{13} \mathrm{C}\left\{{ }^{1} \mathrm{H}\right\}$ NMR $\left(126 \mathrm{MHz}, \mathrm{CDCl}_{3}, 298 \mathrm{~K}\right)$

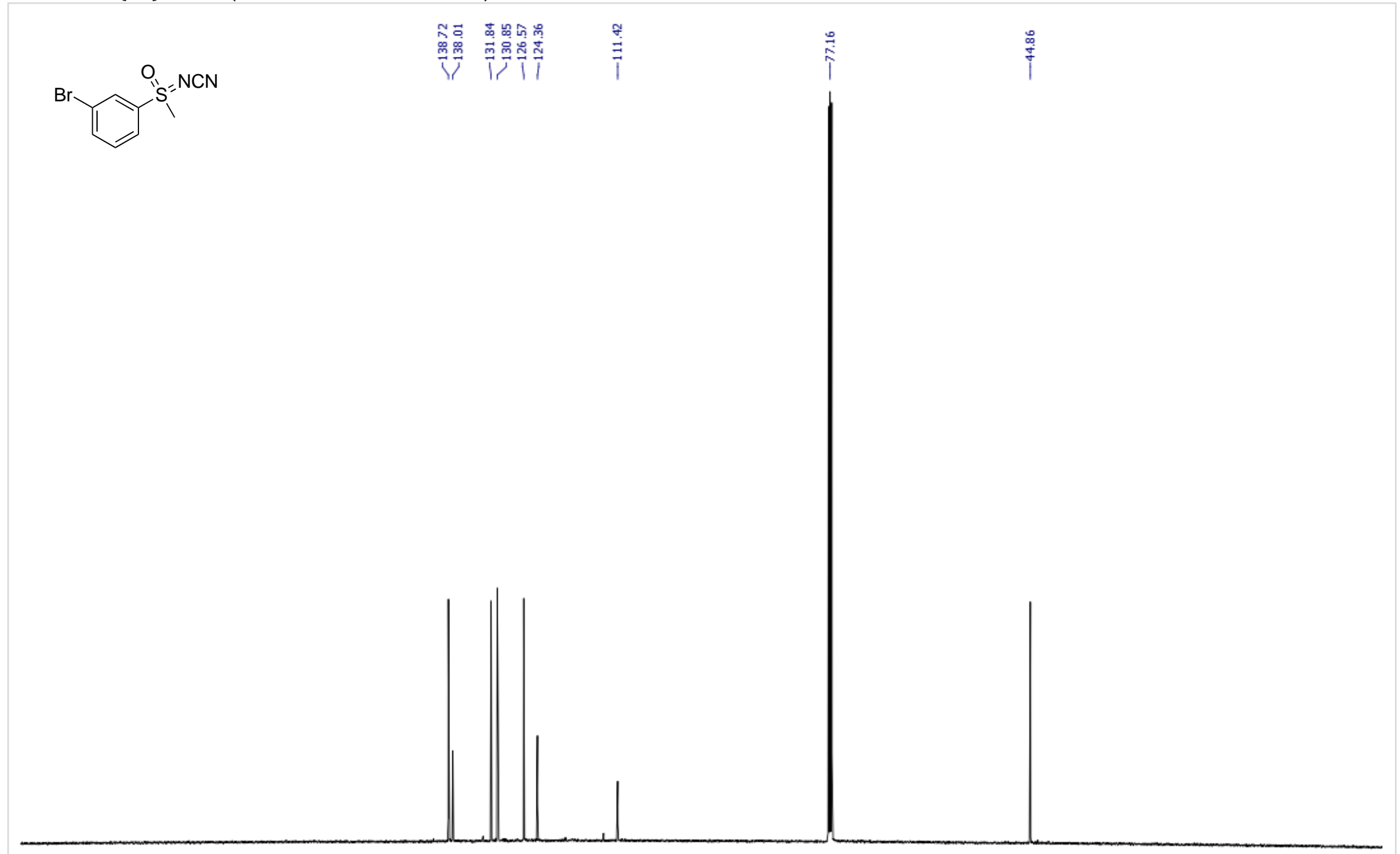


$N$-((3-chlorophenyl)(methyl)(oxo)- $\lambda^{6}$-sulfaneylidene)cyanamide (5i)

${ }^{1} \mathrm{H}$ NMR $\left(500 \mathrm{MHz}, \mathrm{CDCl}_{3}, 298 \mathrm{~K}\right)$

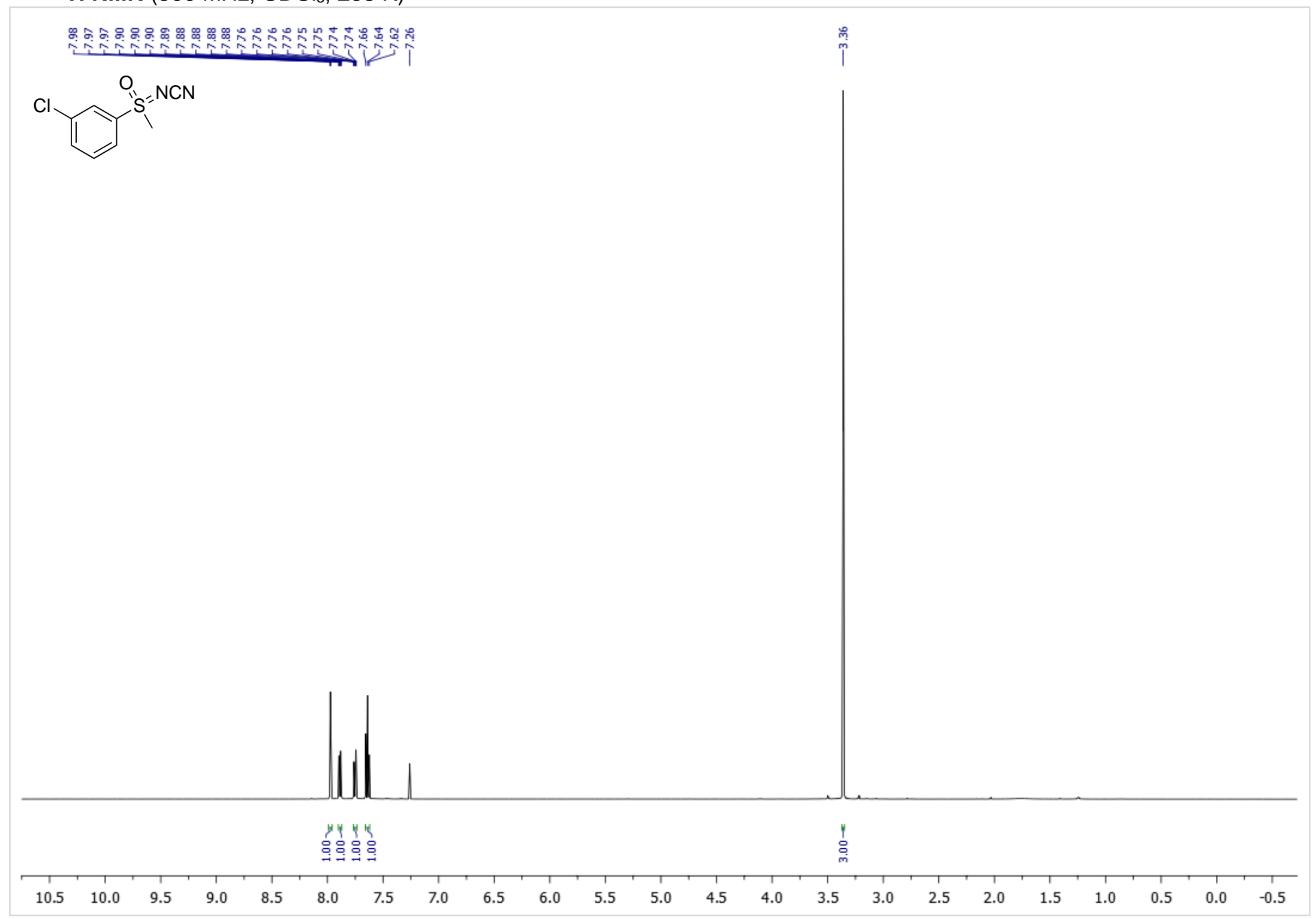


${ }^{13} \mathrm{C}\left\{{ }^{1} \mathrm{H}\right\}$ NMR $\left(101 \mathrm{MHz}, \mathrm{CDCl}_{3}, 298 \mathrm{~K}\right)$

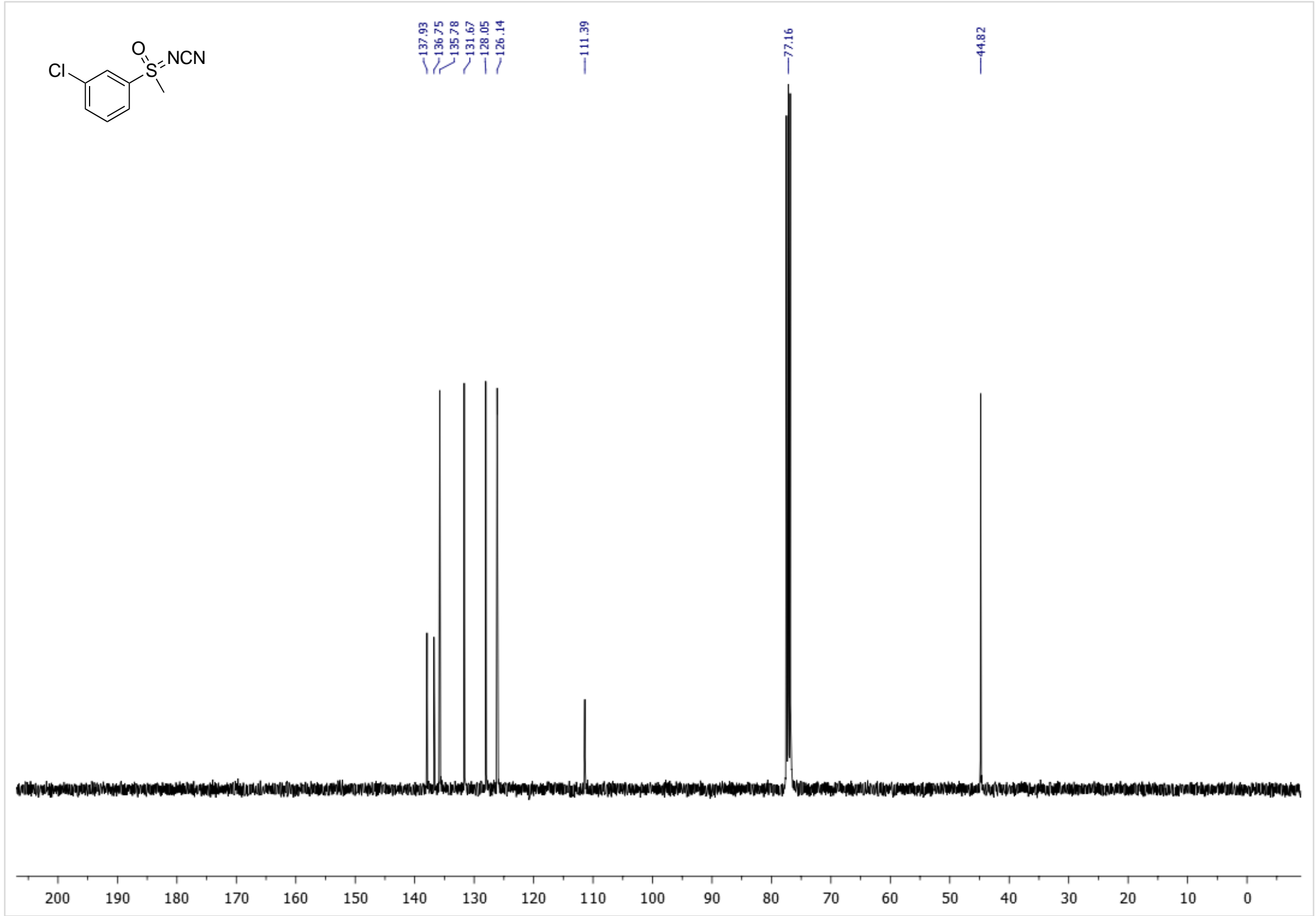


$N$-((3,5-dichlorophenyl)(methyl)(oxo)- $\lambda^{6}$-sulfaneylidene)cyanamide (5I)

${ }^{1} \mathrm{H}$ NMR $\left(500 \mathrm{MHz}, \mathrm{CDCl}_{3}, 298 \mathrm{~K}\right)$
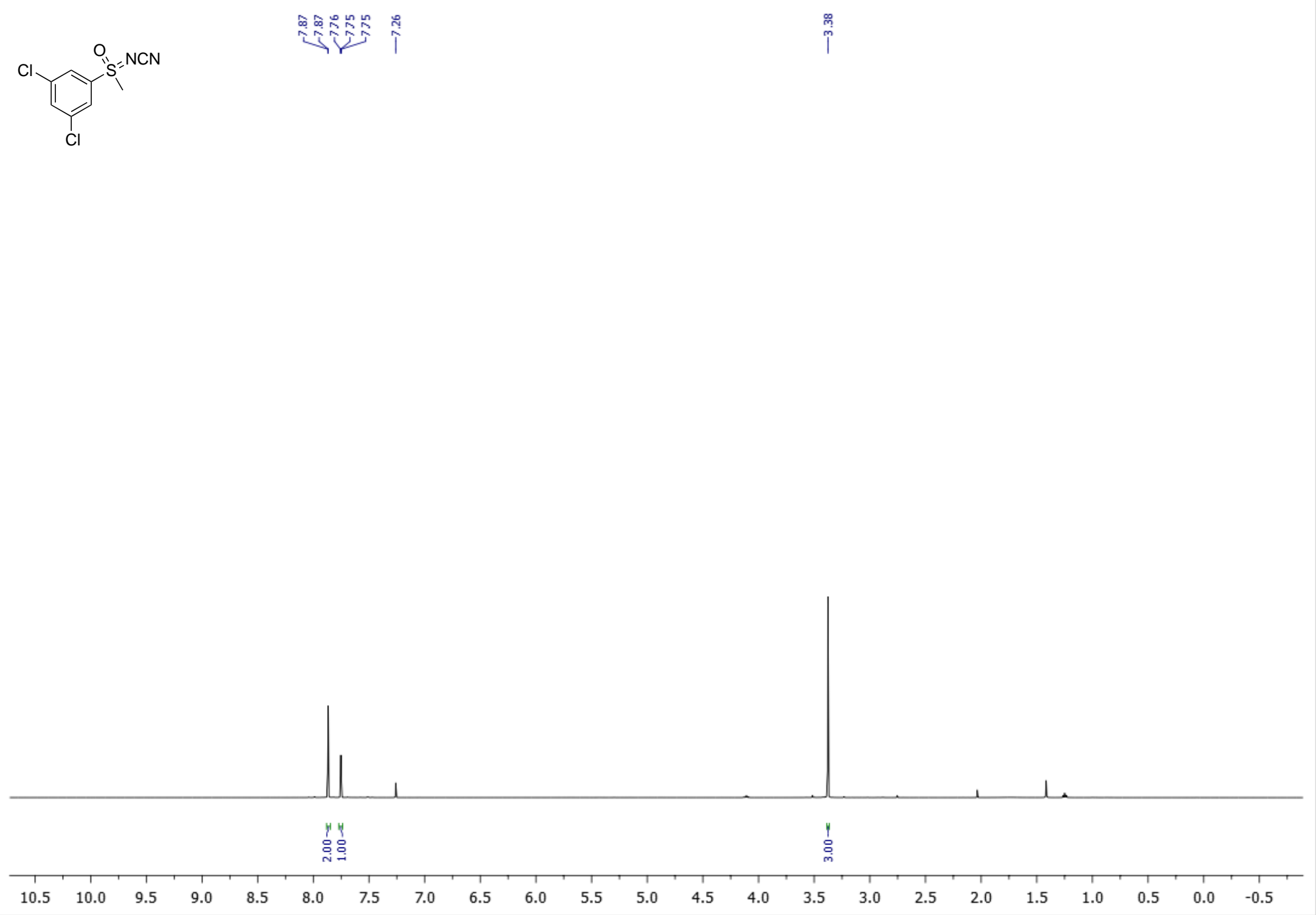
${ }^{13} \mathrm{C}\left\{{ }^{1} \mathrm{H}\right\}$ NMR $\left(126 \mathrm{MHz}, \mathrm{CDCl}_{3}, 298 \mathrm{~K}\right)$

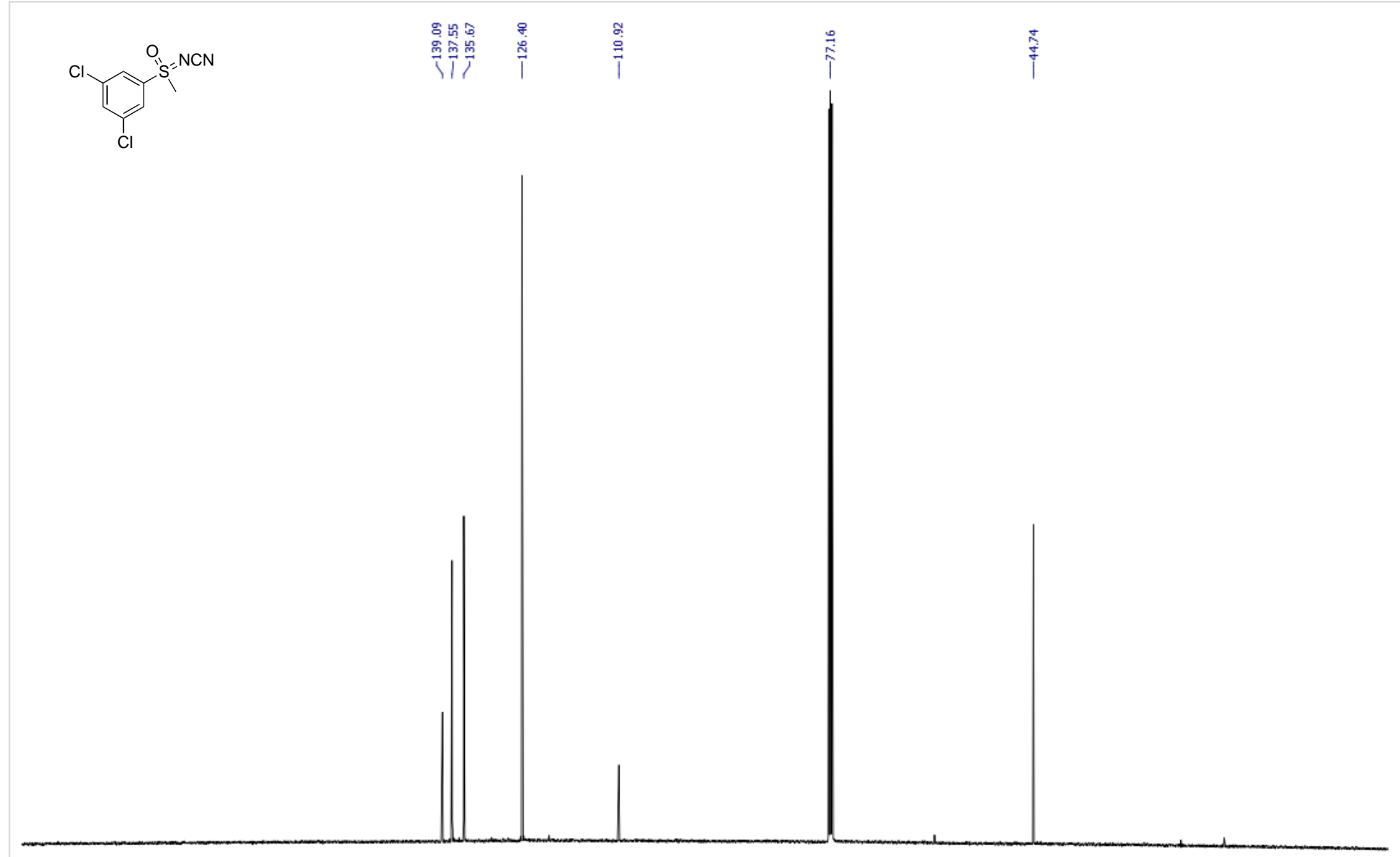


$N$-(ethyl(oxo)(phenyl)- $\lambda^{6}$-sulfaneylidene)cyanamide $(5 \mathrm{~m})$

${ }^{1} \mathrm{H}$ NMR $\left(500 \mathrm{MHz}, \mathrm{CDCl}_{3}, 298 \mathrm{~K}\right)$

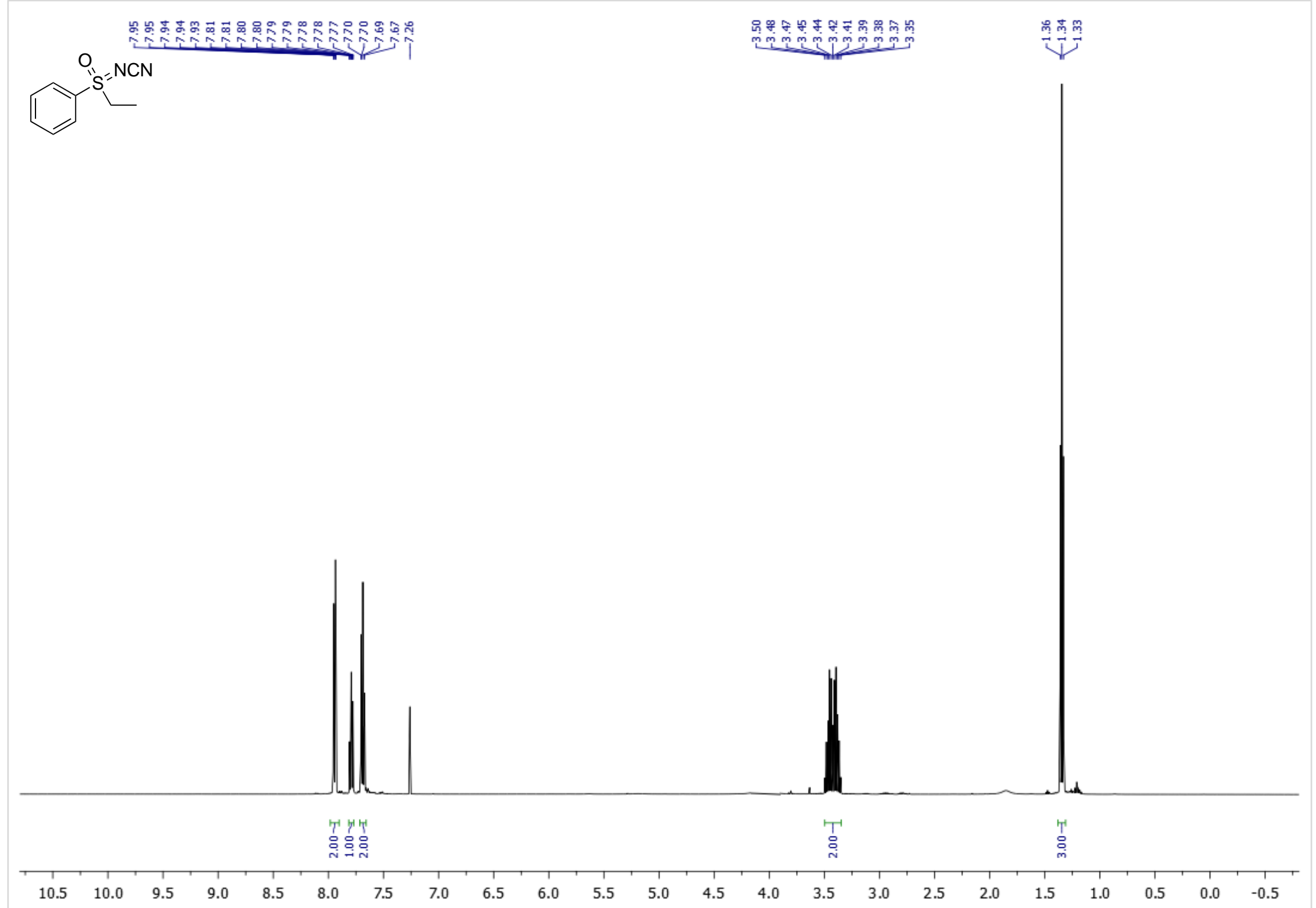


${ }^{13} \mathrm{C}\left\{{ }^{1} \mathrm{H}\right\}$ NMR $\left(126 \mathrm{MHz}, \mathrm{CDCl}_{3}, 298 \mathrm{~K}\right)$

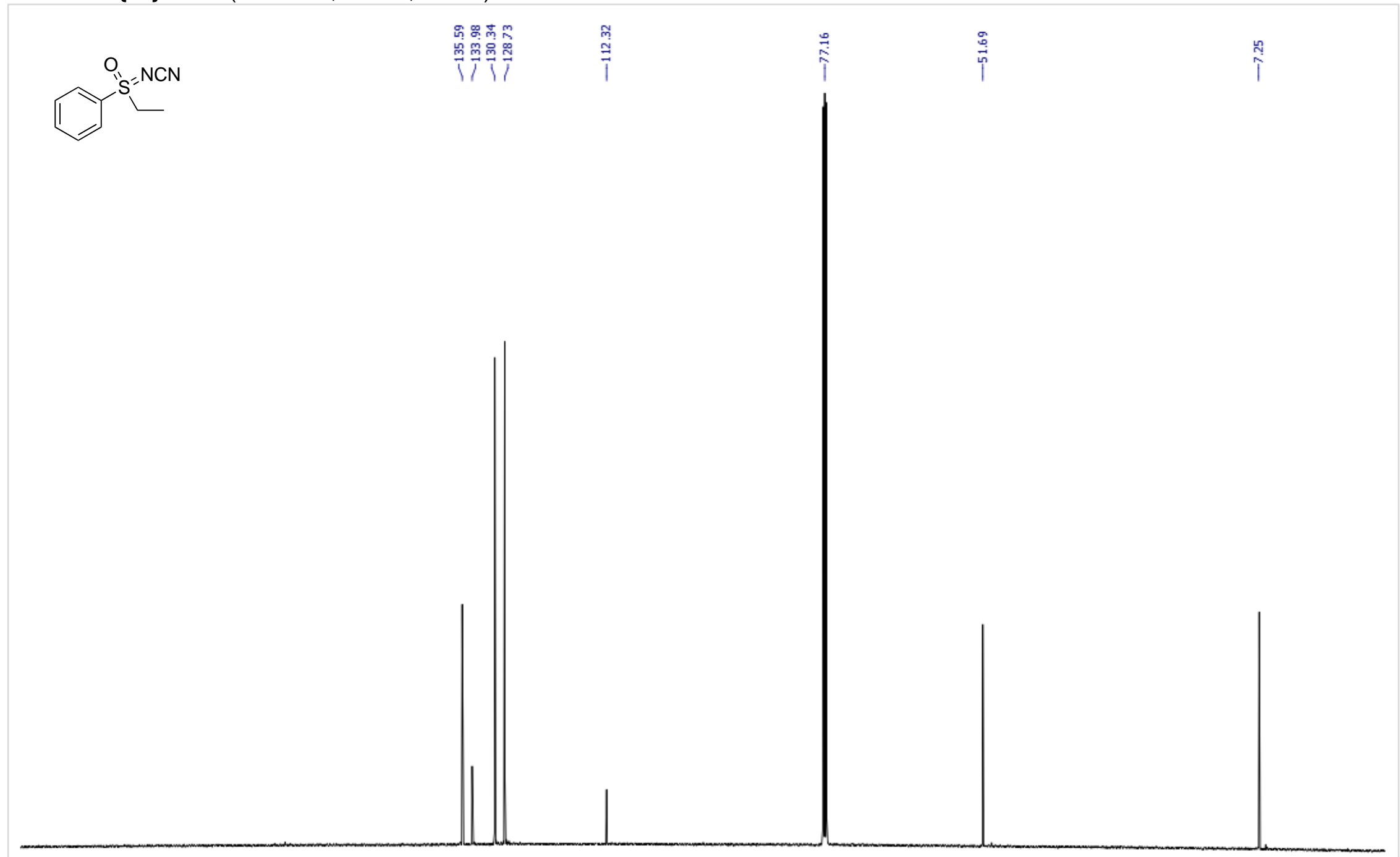


$N$-(oxo(phenyl)(propyl)- $\lambda^{6}$-sulfaneylidene)cyanamide (5n)

${ }^{1} \mathrm{H}$ NMR $\left(500 \mathrm{MHz}, \mathrm{CDCl}_{3}, 298 \mathrm{~K}\right)$

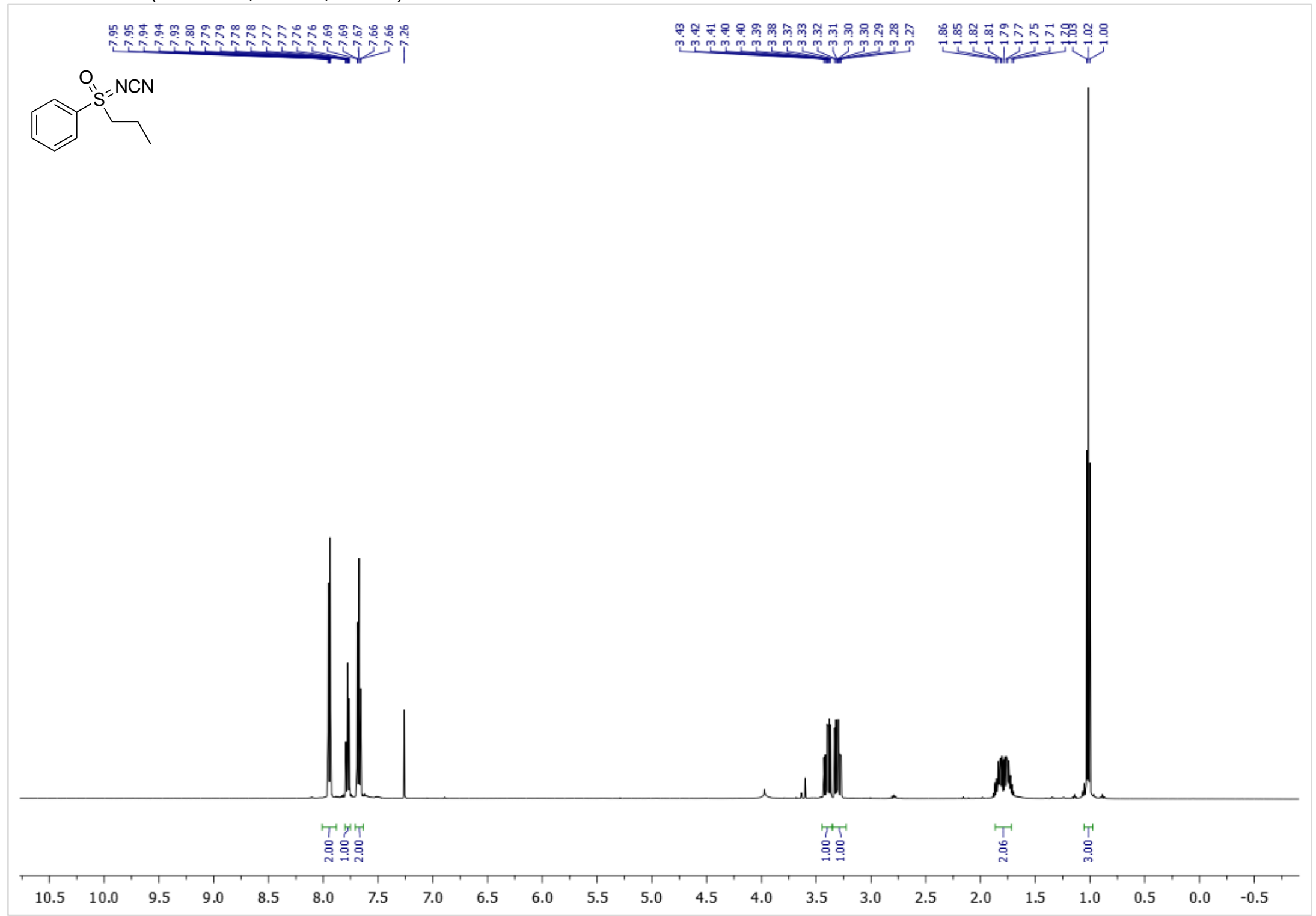


${ }^{13} \mathrm{C}\left\{{ }^{1} \mathrm{H}\right\}$ NMR $\left(126 \mathrm{MHz}, \mathrm{CDCl}_{3}, 298 \mathrm{~K}\right)$

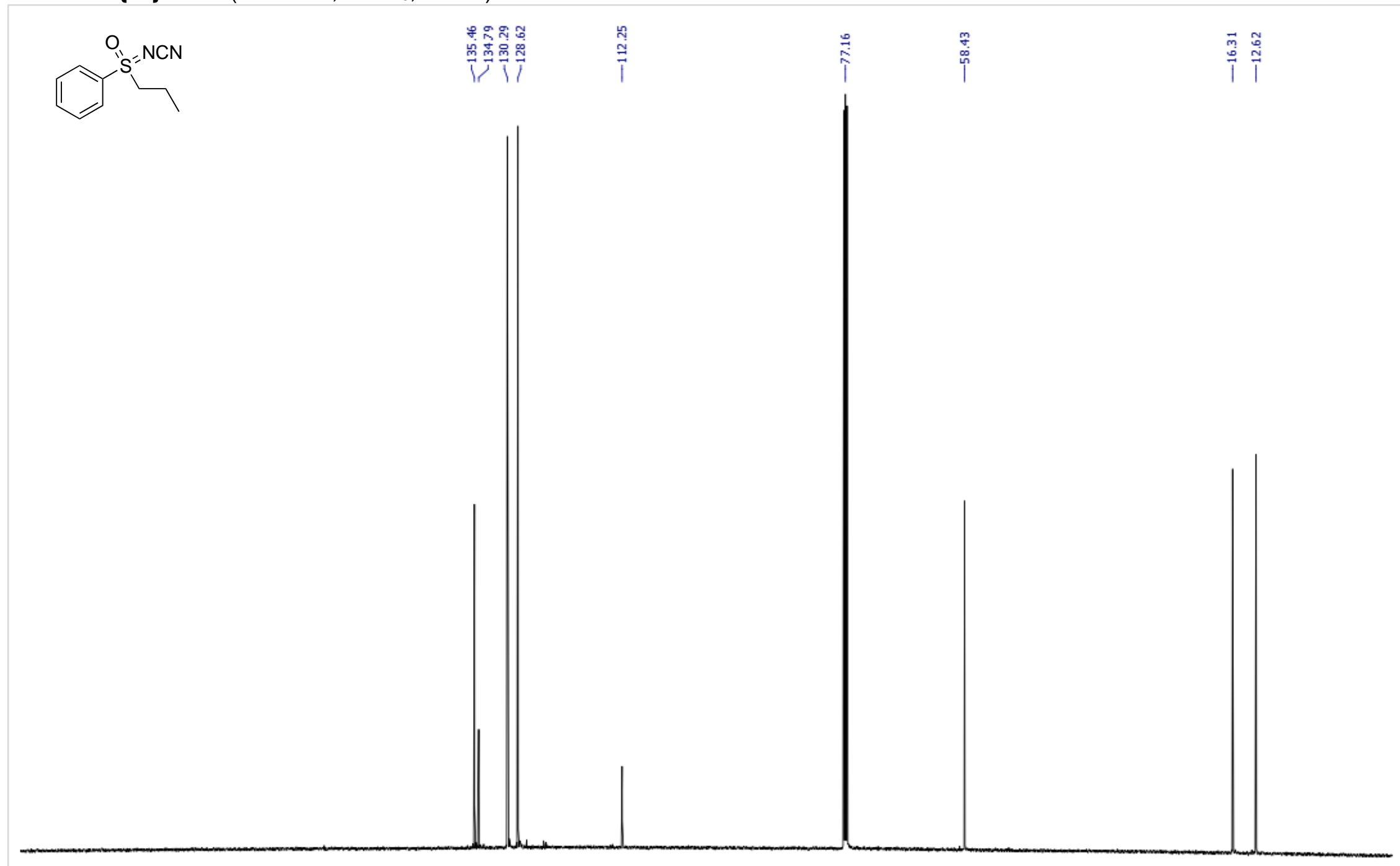

200

$\begin{array}{llll}190 & 180 & 170 & 160\end{array}$

150

140

130

$20 \quad 110$

100

$90 \quad 80$

$70 \quad 60$

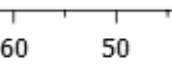

40 
$\boldsymbol{N}$-(cyclopropyl(oxo)(phenyl)- $\lambda^{6}$-sulfaneylidene)cyanamide (50)

${ }^{1} \mathrm{H}$ NMR $\left(500 \mathrm{MHz}, \mathrm{CDCl}_{3}, 298 \mathrm{~K}\right)$

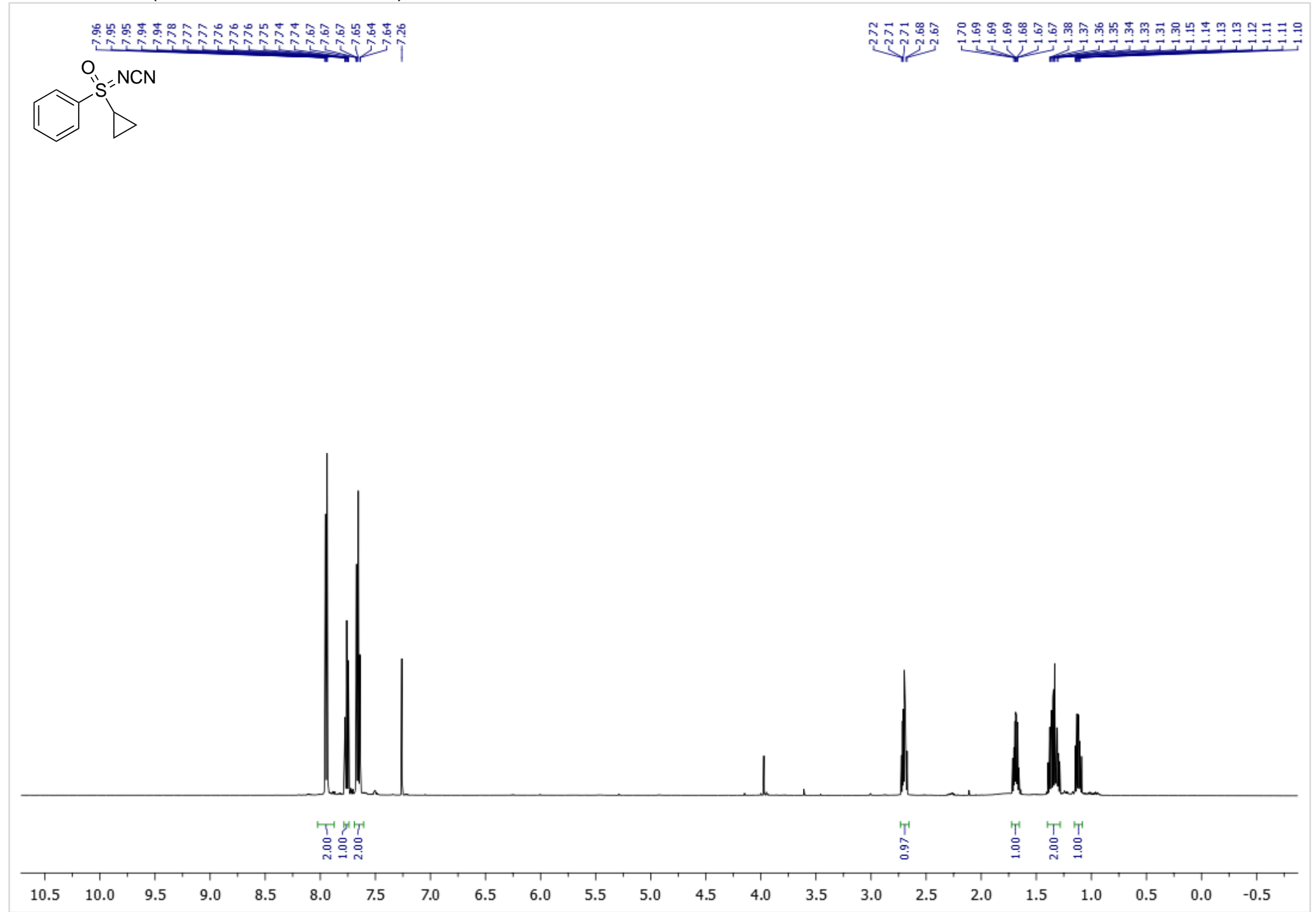


${ }^{13} \mathrm{C}\left\{{ }^{1} \mathrm{H}\right\}$ NMR $\left(126 \mathrm{MHz}, \mathrm{CDCl}_{3}, 298 \mathrm{~K}\right)$

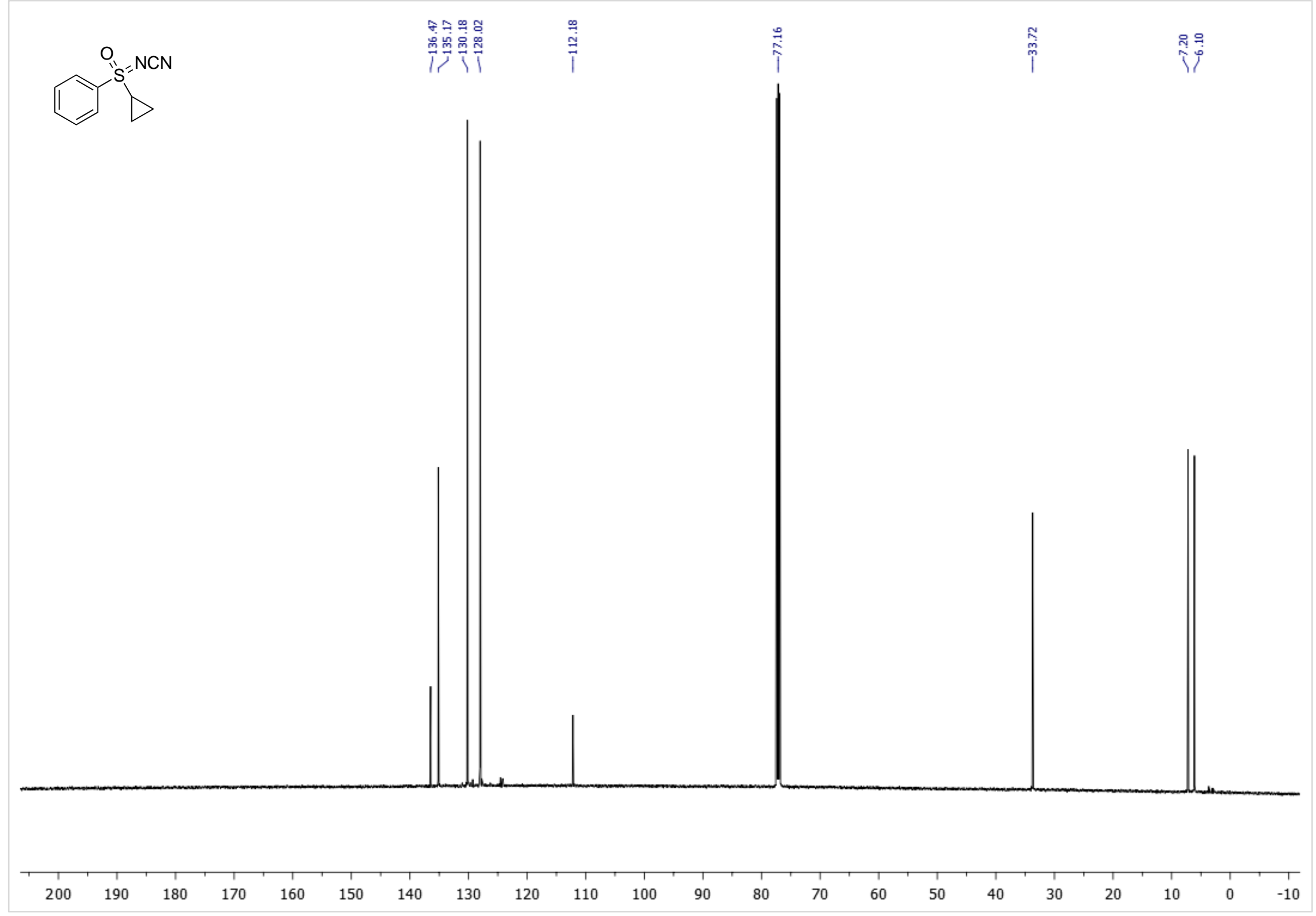

S145 
$N$-(methyl(oxo)(pyridin-2-yl)- $\lambda^{6}$-sulfaneylidene)cyanamide (5q)

${ }^{1} \mathrm{H}$ NMR $\left(500 \mathrm{MHz}, \mathrm{CDCl}_{3}, 298 \mathrm{~K}\right)$

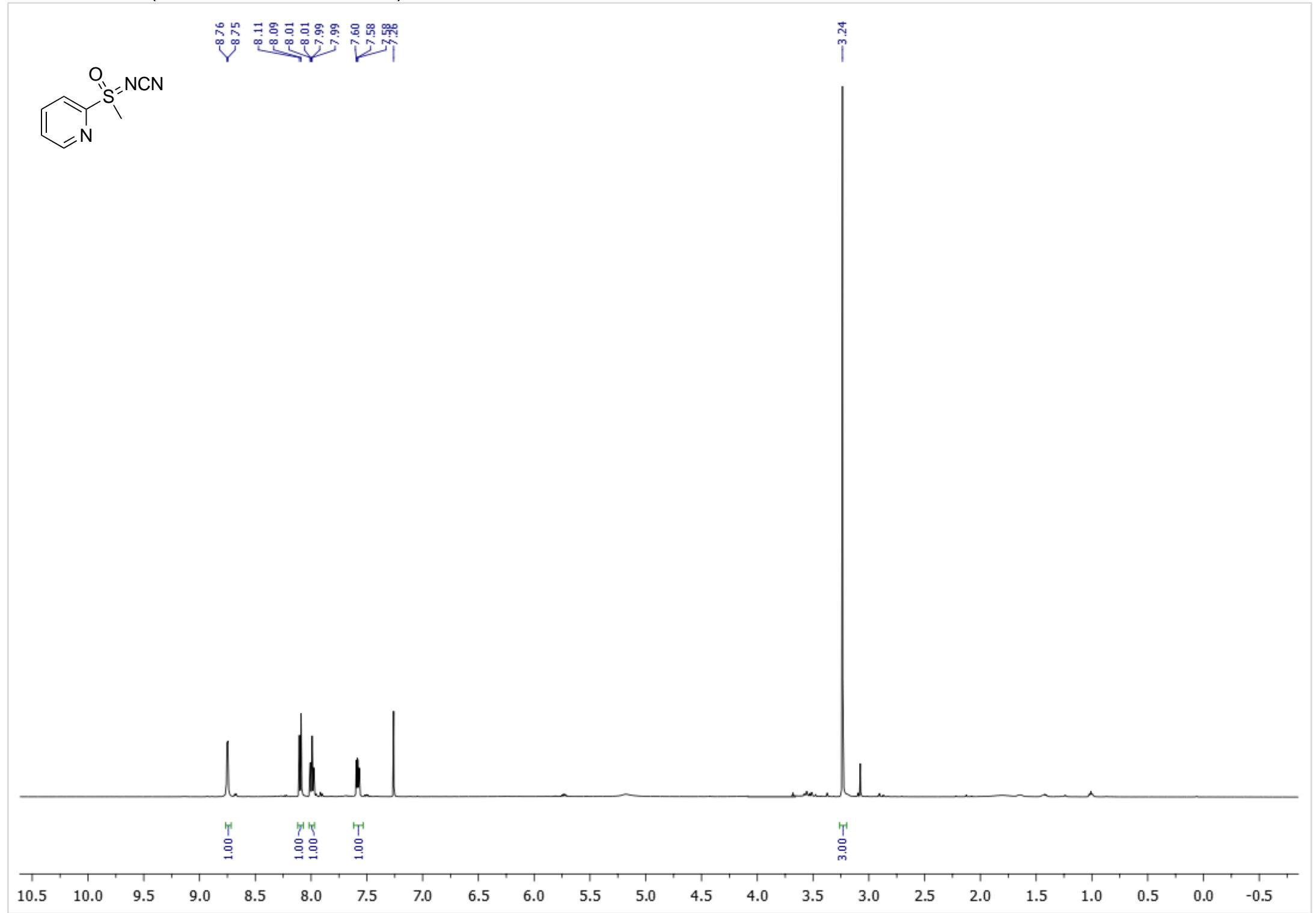


${ }^{13} \mathrm{C}\left\{{ }^{1} \mathrm{H}\right\}$ NMR $\left(126 \mathrm{MHz}, \mathrm{CDCl}_{3}, 298 \mathrm{~K}\right)$

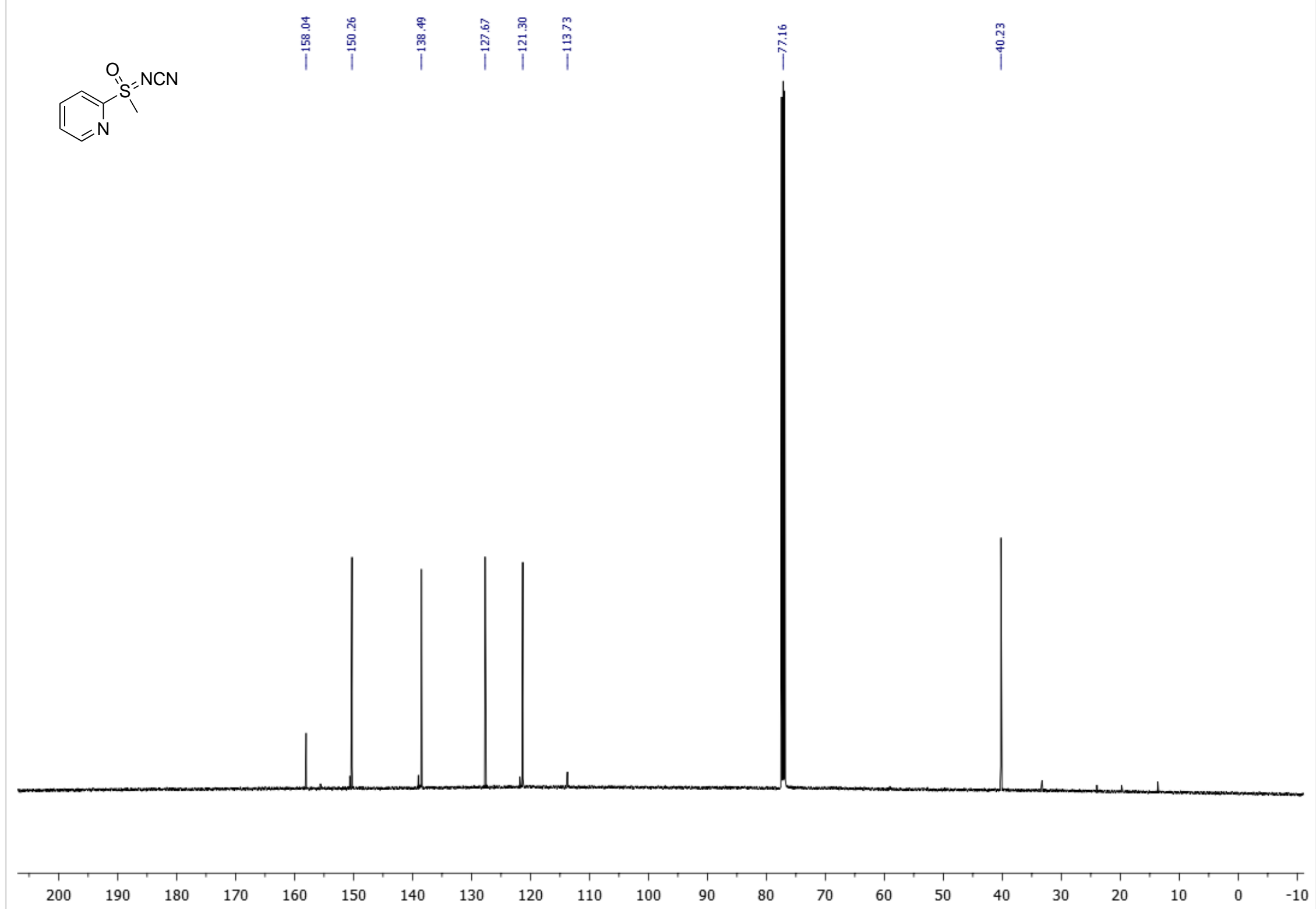


$N$-(oxodiphenyl- $\lambda^{6}$-sulfaneylidene)cyanamide (5p)

${ }^{1} \mathrm{H}$ NMR $\left(500 \mathrm{MHz}, \mathrm{CDCl}_{3}, 298 \mathrm{~K}\right)$

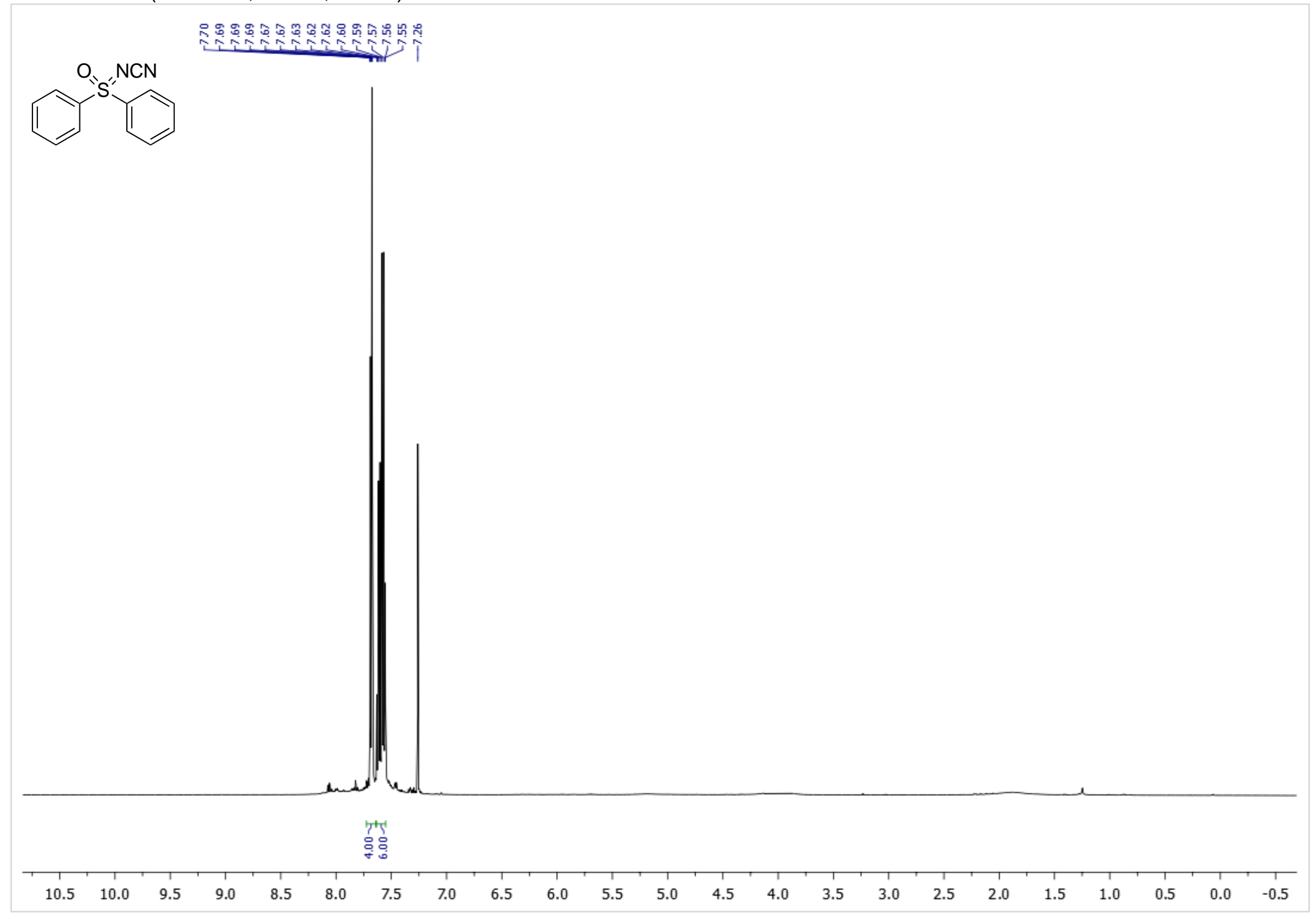


${ }^{13} \mathrm{C}\left\{{ }^{1} \mathrm{H}\right\}$ NMR $\left(126 \mathrm{MHz}, \mathrm{CDCl}_{3}, 298 \mathrm{~K}\right)$

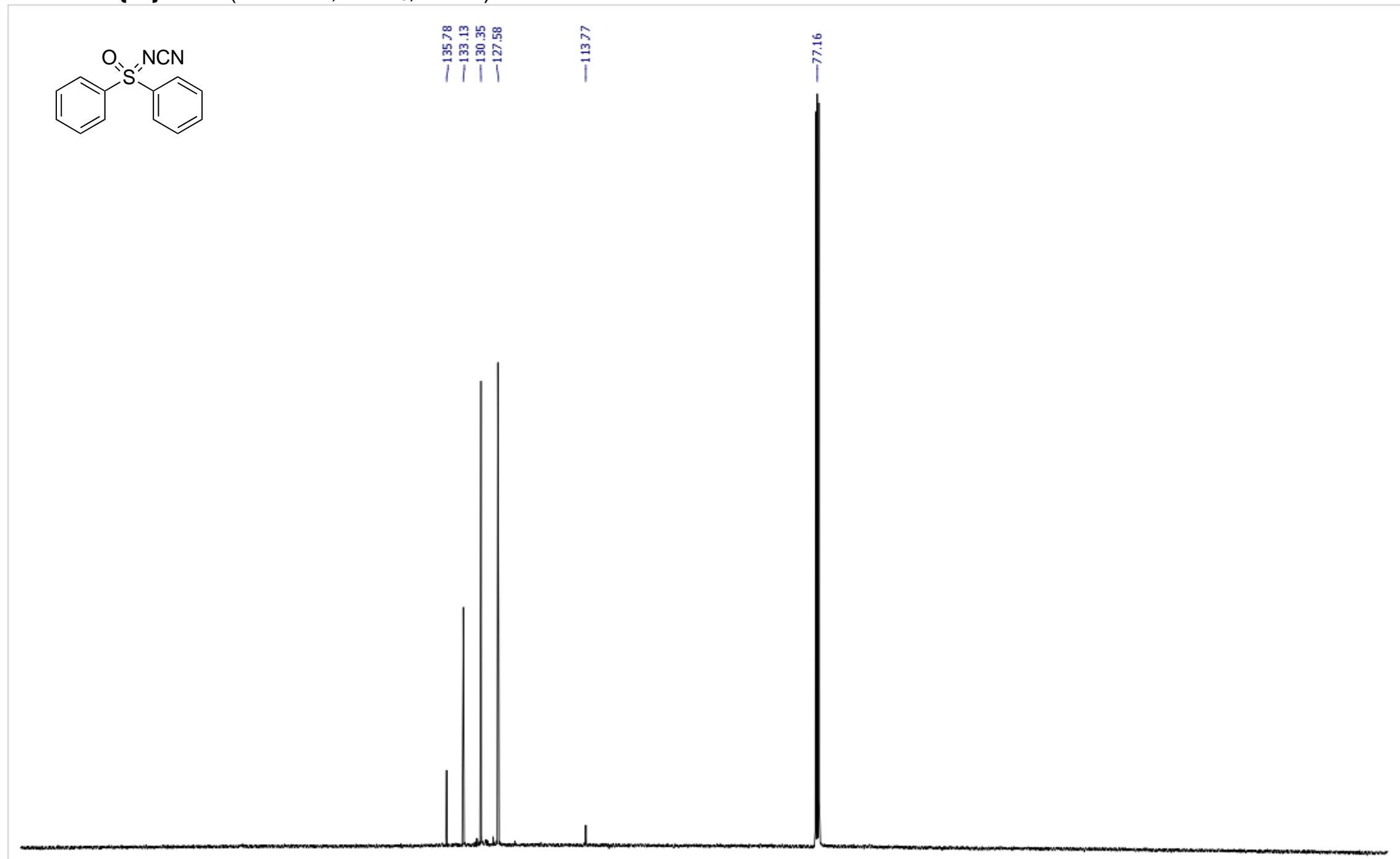

$$
200
$$

$\begin{array}{llll}190 & 180 & 170 & 160\end{array}$

150

$140 \quad 130$

120

$110 \quad 100$

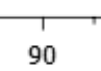

$80 \quad 70$

60 
$\mathrm{N}$-(1-oxidotetrahydro-2 $\mathrm{H}-1 \lambda^{6}$-thiopyran-1-ylidene)cyanamide (5r)

${ }^{1} \mathrm{H}$ NMR $\left(500 \mathrm{MHz}, \mathrm{CDCl}_{3}, 298 \mathrm{~K}\right)$

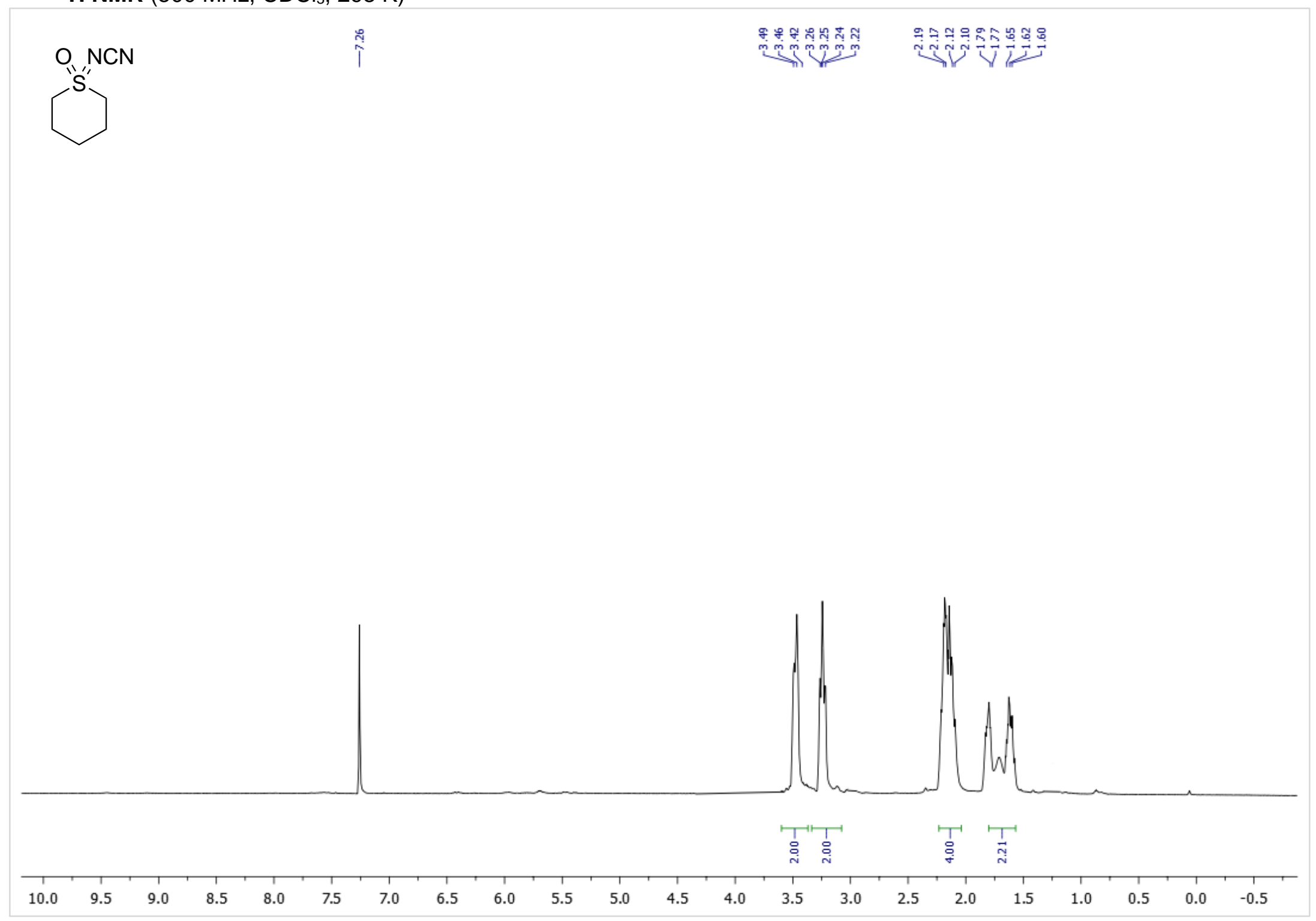


${ }^{13} \mathrm{C}\left\{{ }^{1} \mathrm{H}\right\}$ NMR $\left(126 \mathrm{MHz}, \mathrm{CDCl}_{3}, 298 \mathrm{~K}\right)$

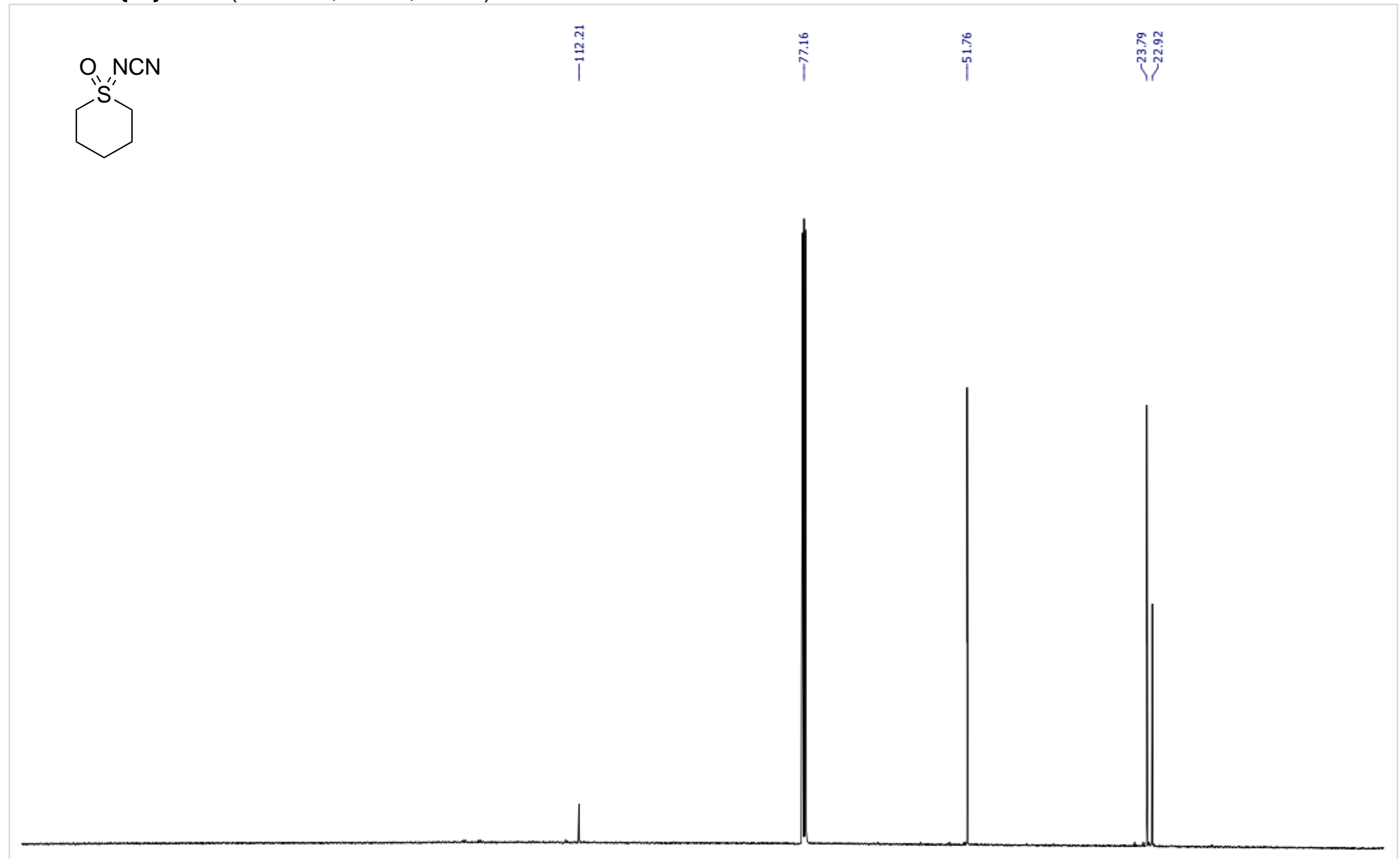


${ }^{1} \mathrm{H}$ NMR $\left(500 \mathrm{MHz}, \mathrm{CDCl}_{3}, 298 \mathrm{~K}\right)$

imino(methyl)(phenyl)- $\lambda^{6}$-sulfanone (6a)

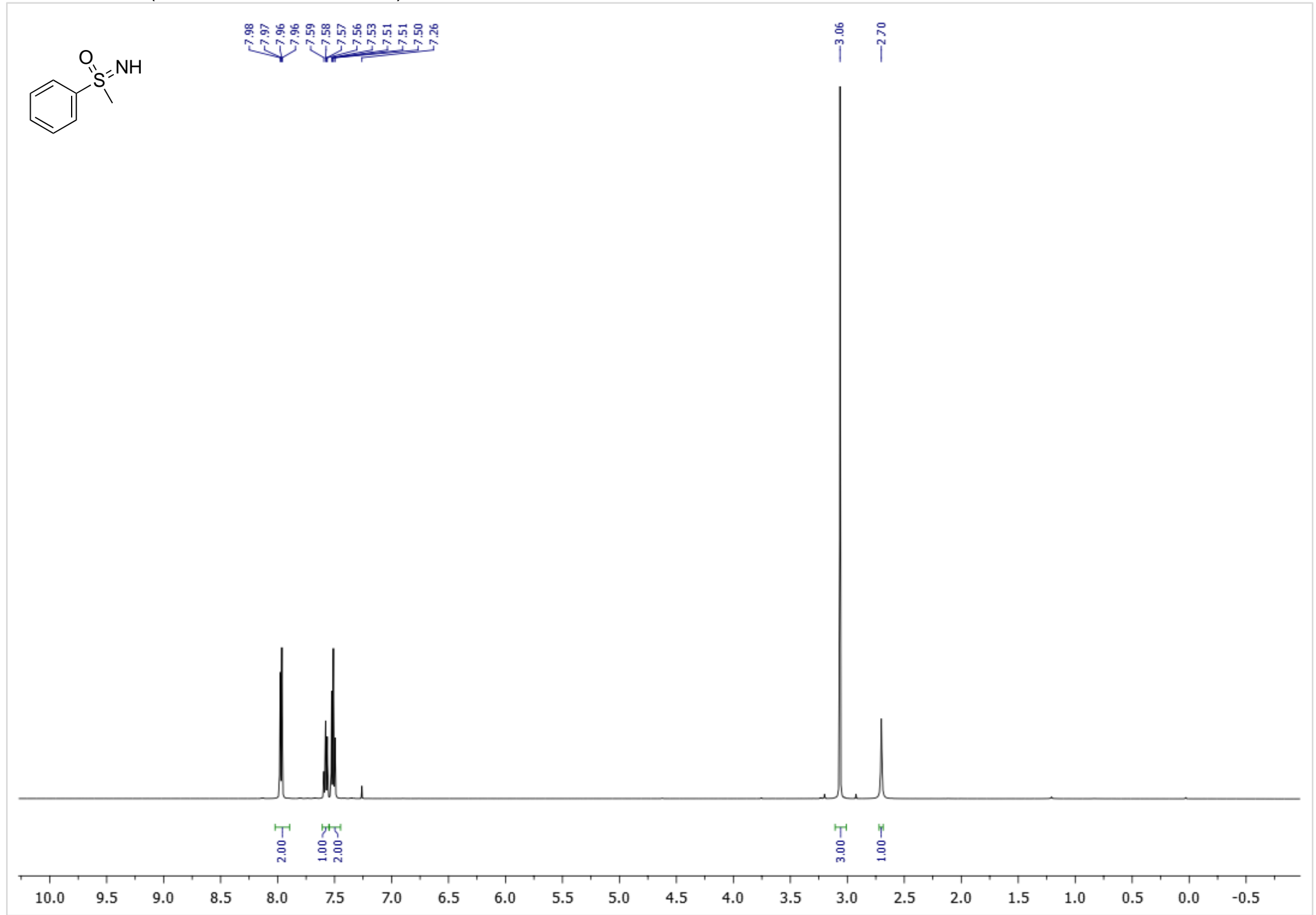

S152 
${ }^{13} \mathrm{C}\left\{{ }^{1} \mathrm{H}\right\}$ NMR $\left(126 \mathrm{MHz}, \mathrm{CDCl}_{3}, 298 \mathrm{~K}\right)$

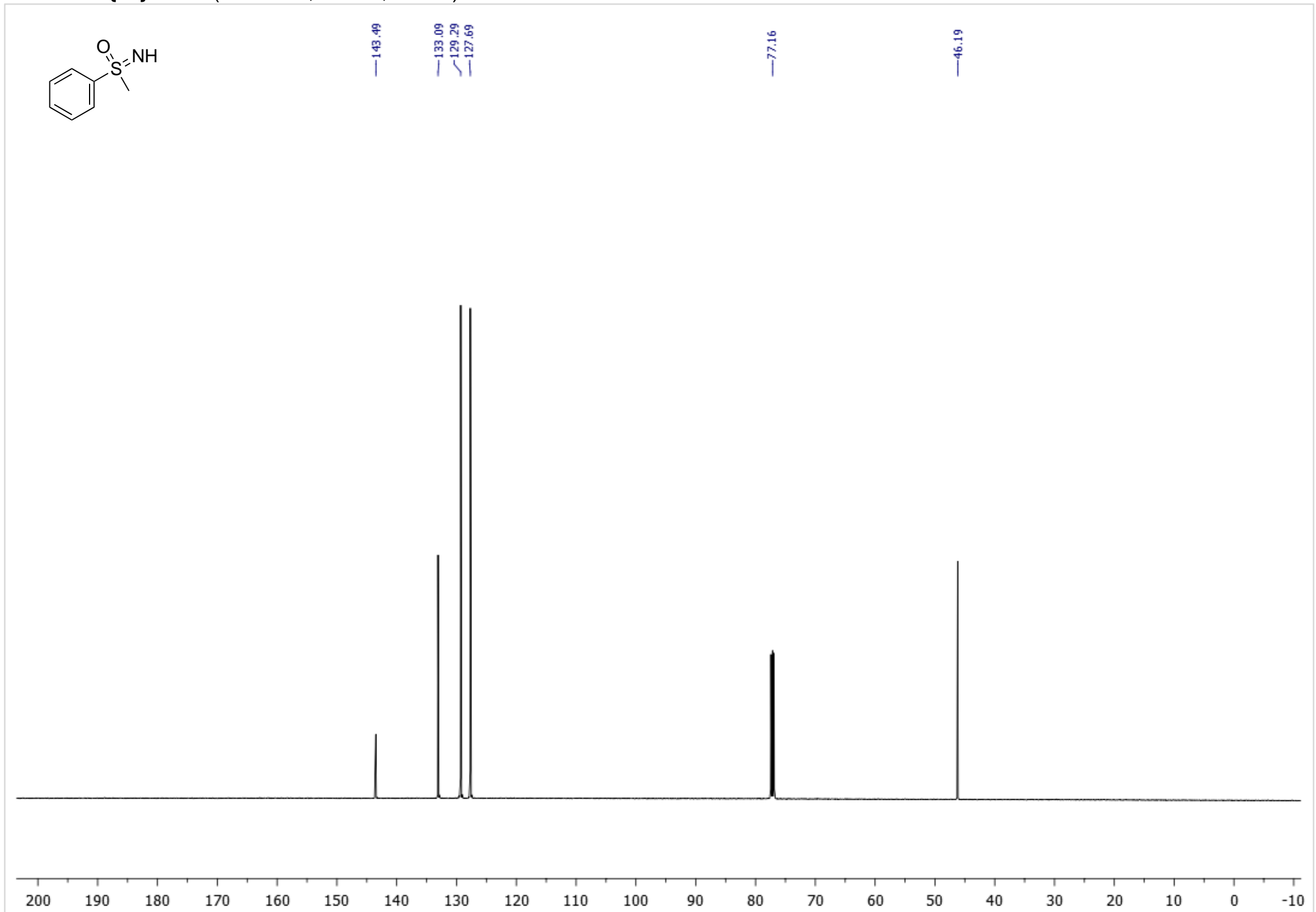

S153 
${ }^{1} \mathbf{H}$ NMR $\left(500 \mathrm{MHz}, \mathrm{CDCl}_{3}, 298 \mathrm{~K}\right)$

imino(methyl)(p-tolyl)- $\lambda^{6}$-sulfanone (6b)

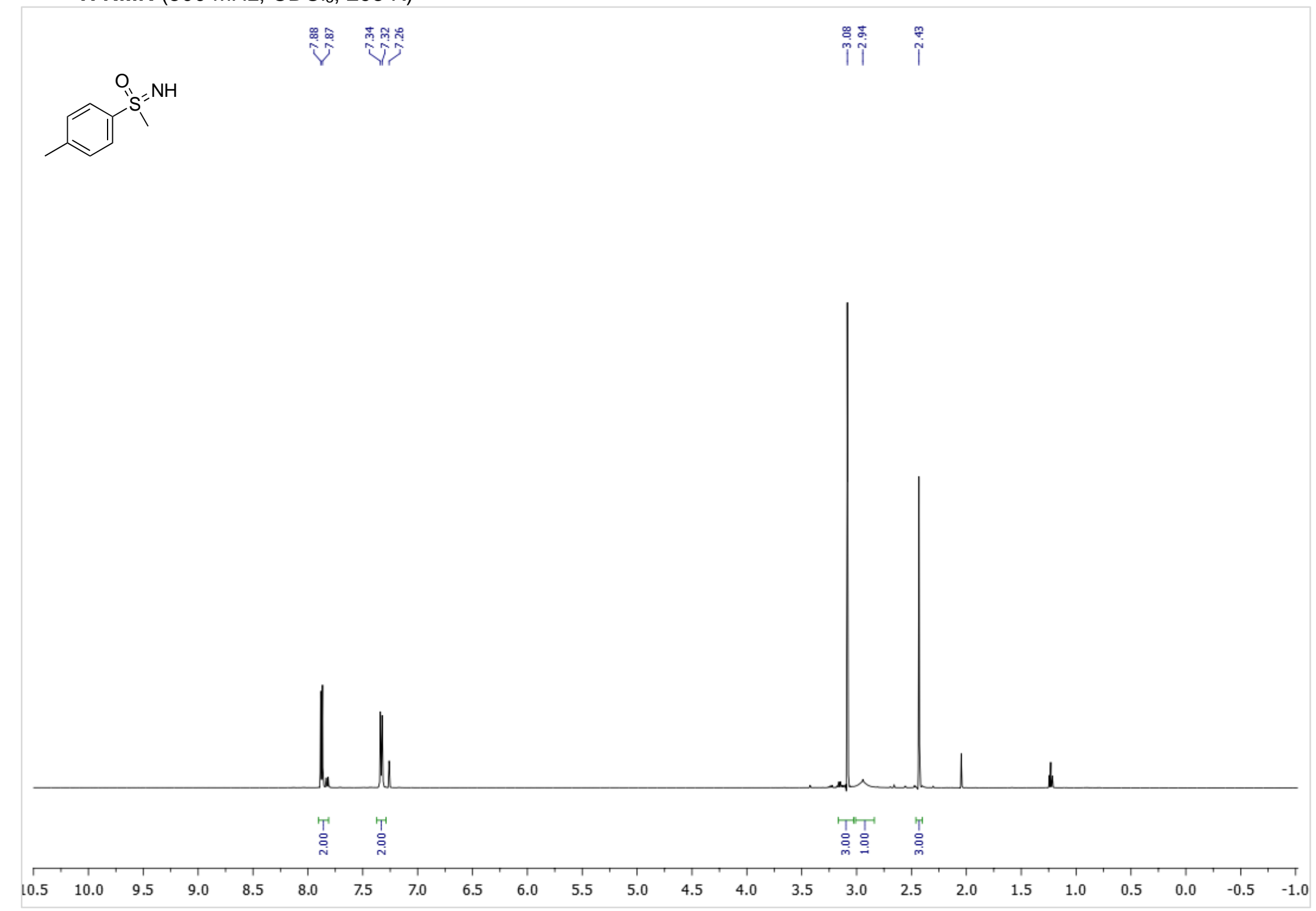


${ }^{13} \mathrm{C}\left\{{ }^{1} \mathrm{H}\right\}$ NMR $\left(126 \mathrm{MHz}, \mathrm{CDCl}_{3}, 298 \mathrm{~K}\right)$

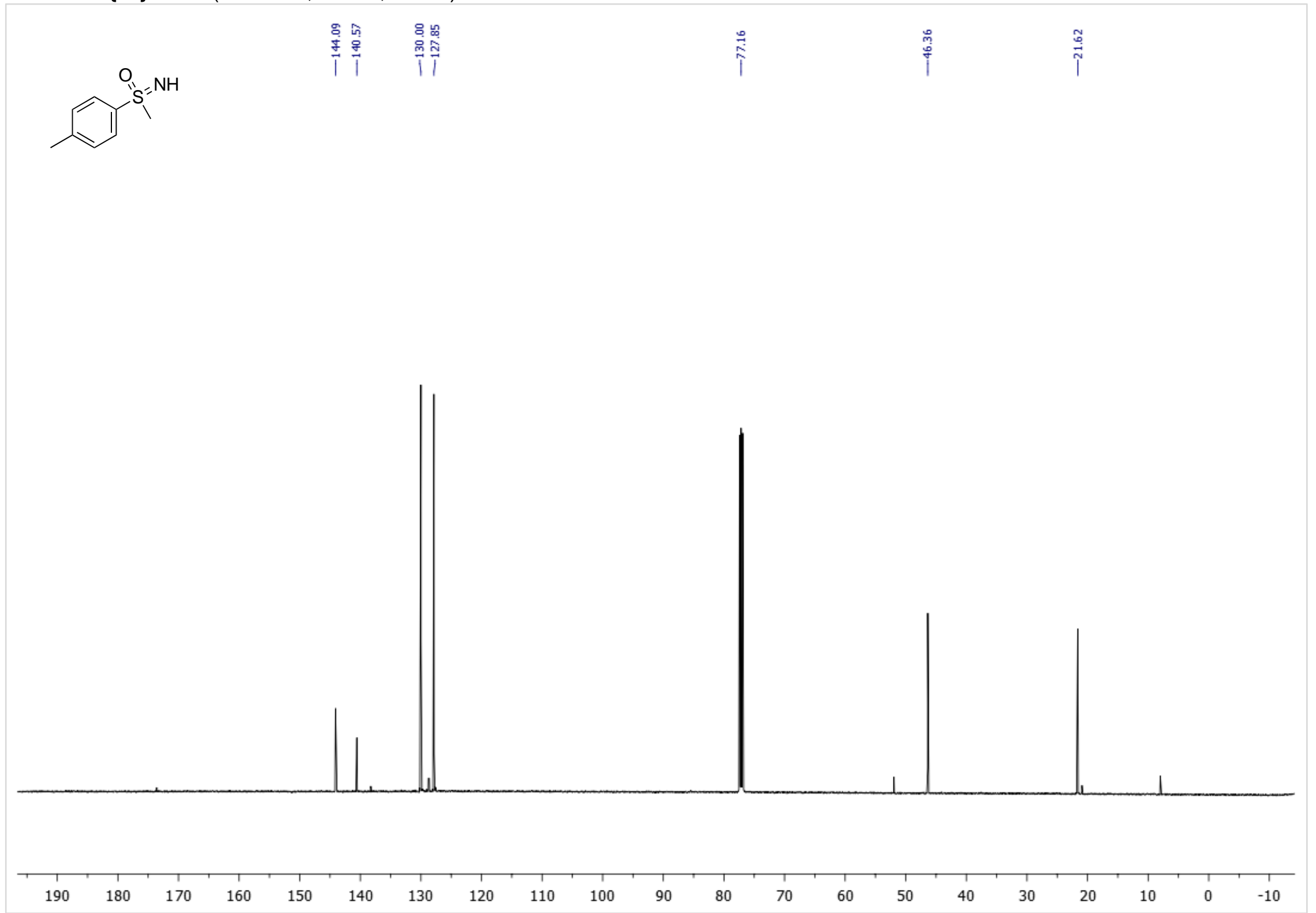

S155 
${ }^{1} \mathrm{H}$ NMR (500 MHz, $\left.\mathrm{CDCl}_{3}, 298 \mathrm{~K}\right)$

ethyl(imino)(phenyl)- $\lambda^{6}$-sulfanone (6c)

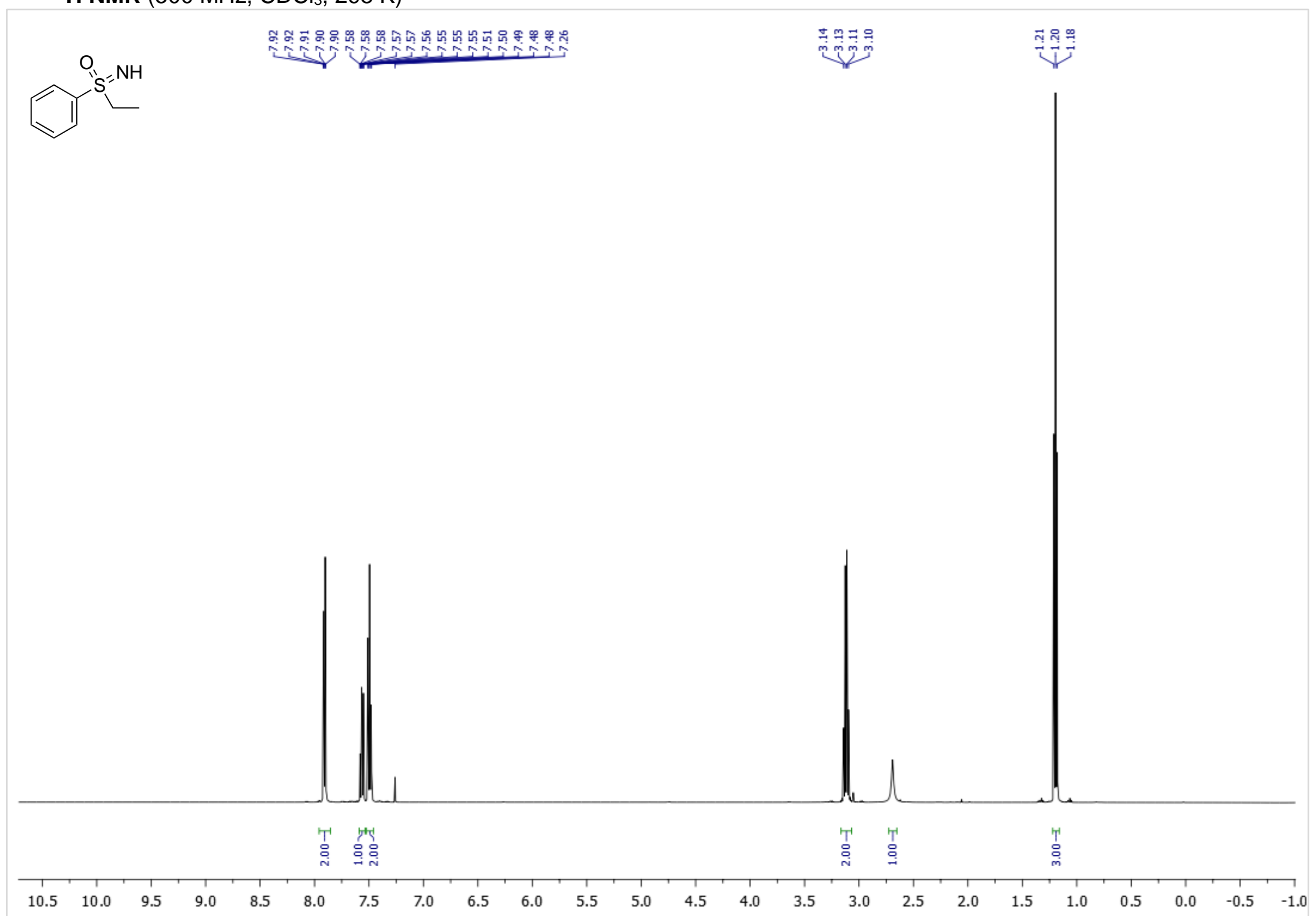


${ }^{13} \mathrm{C}\left\{{ }^{1} \mathrm{H}\right\}$ NMR $\left(126 \mathrm{MHz}, \mathrm{CDCl}_{3}, 298 \mathrm{~K}\right)$

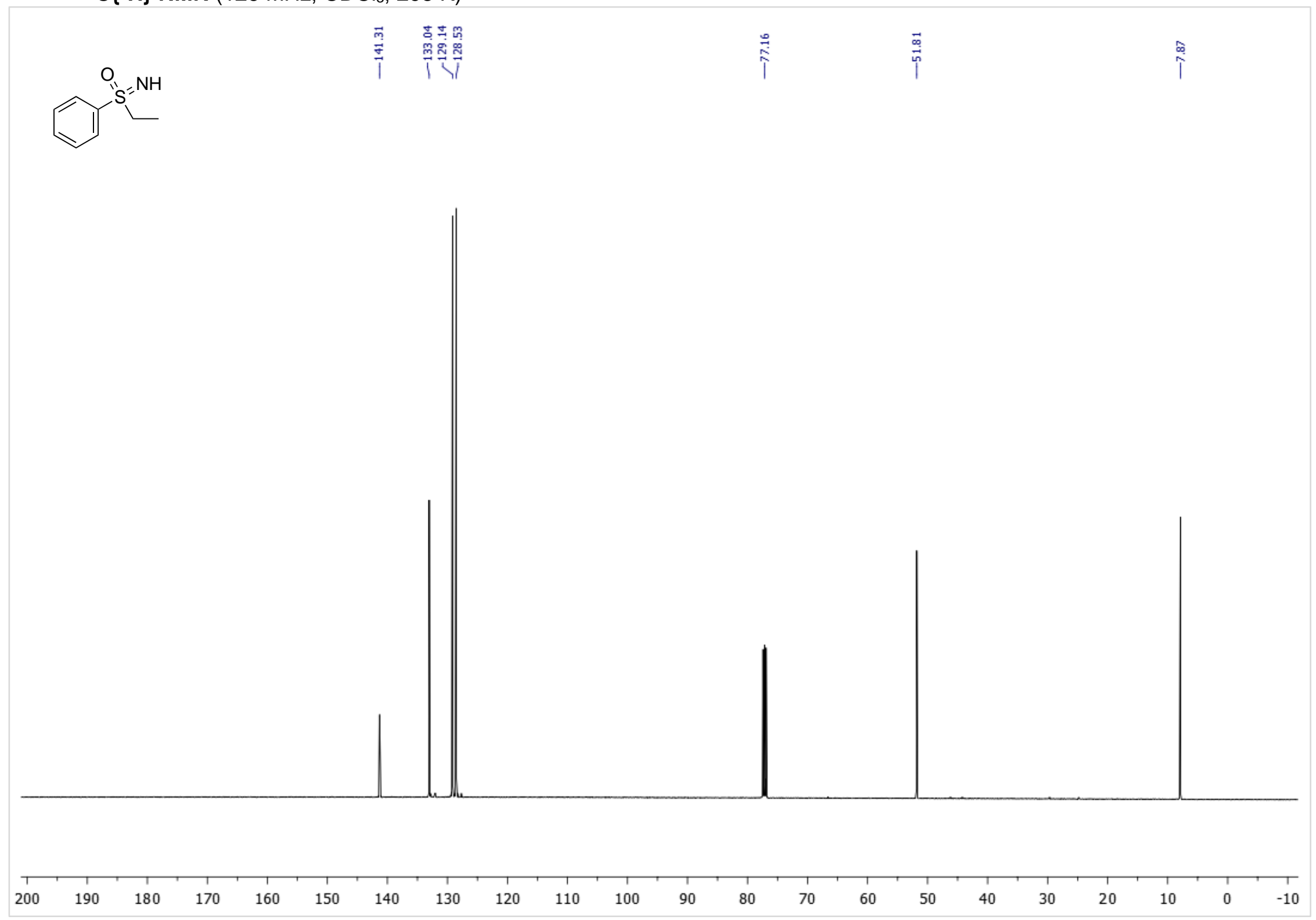




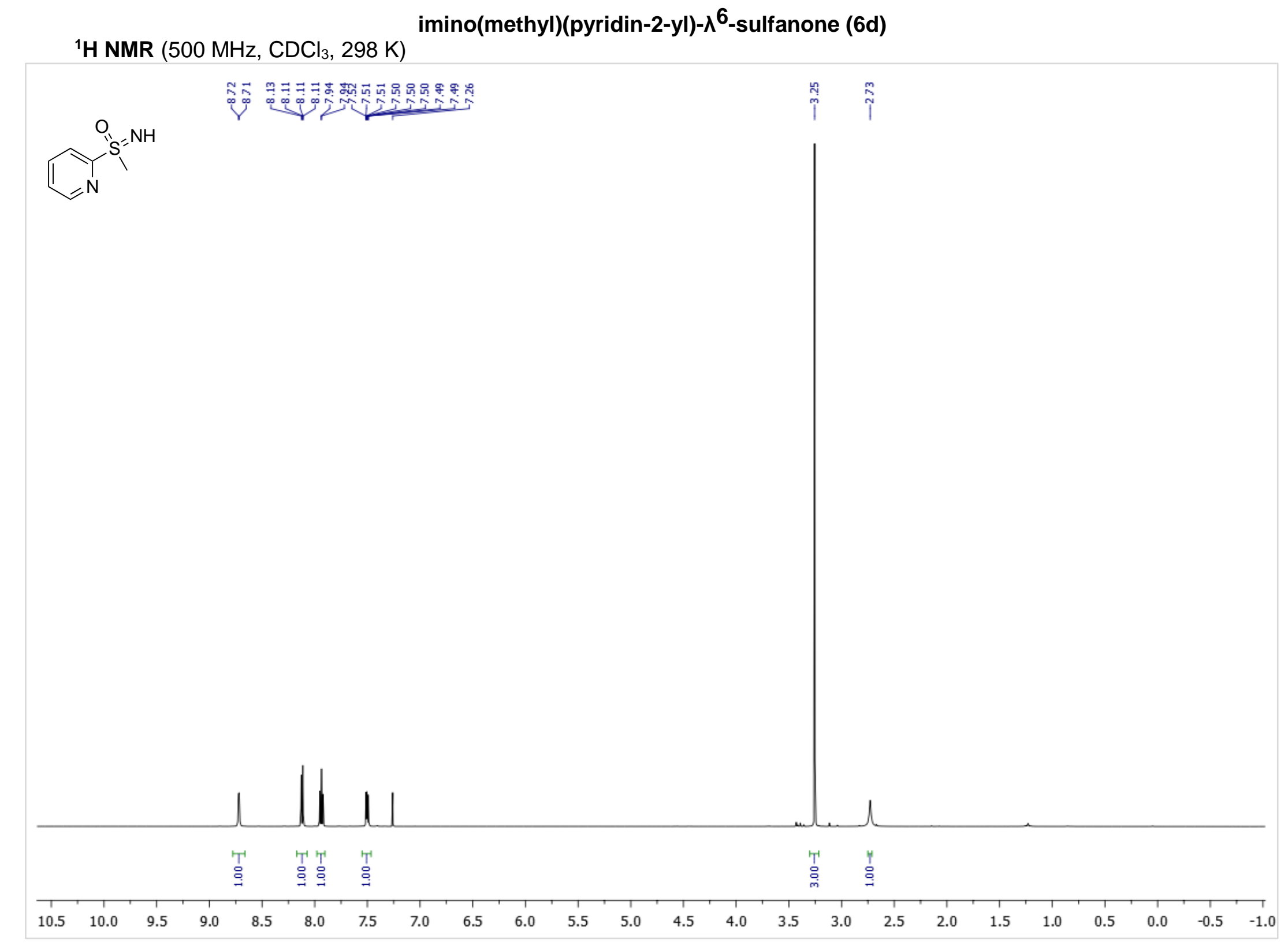


${ }^{13} \mathrm{C}\left\{{ }^{1} \mathrm{H}\right\}$ NMR $\left(126 \mathrm{MHz}, \mathrm{CDCl}_{3}, 298 \mathrm{~K}\right)$

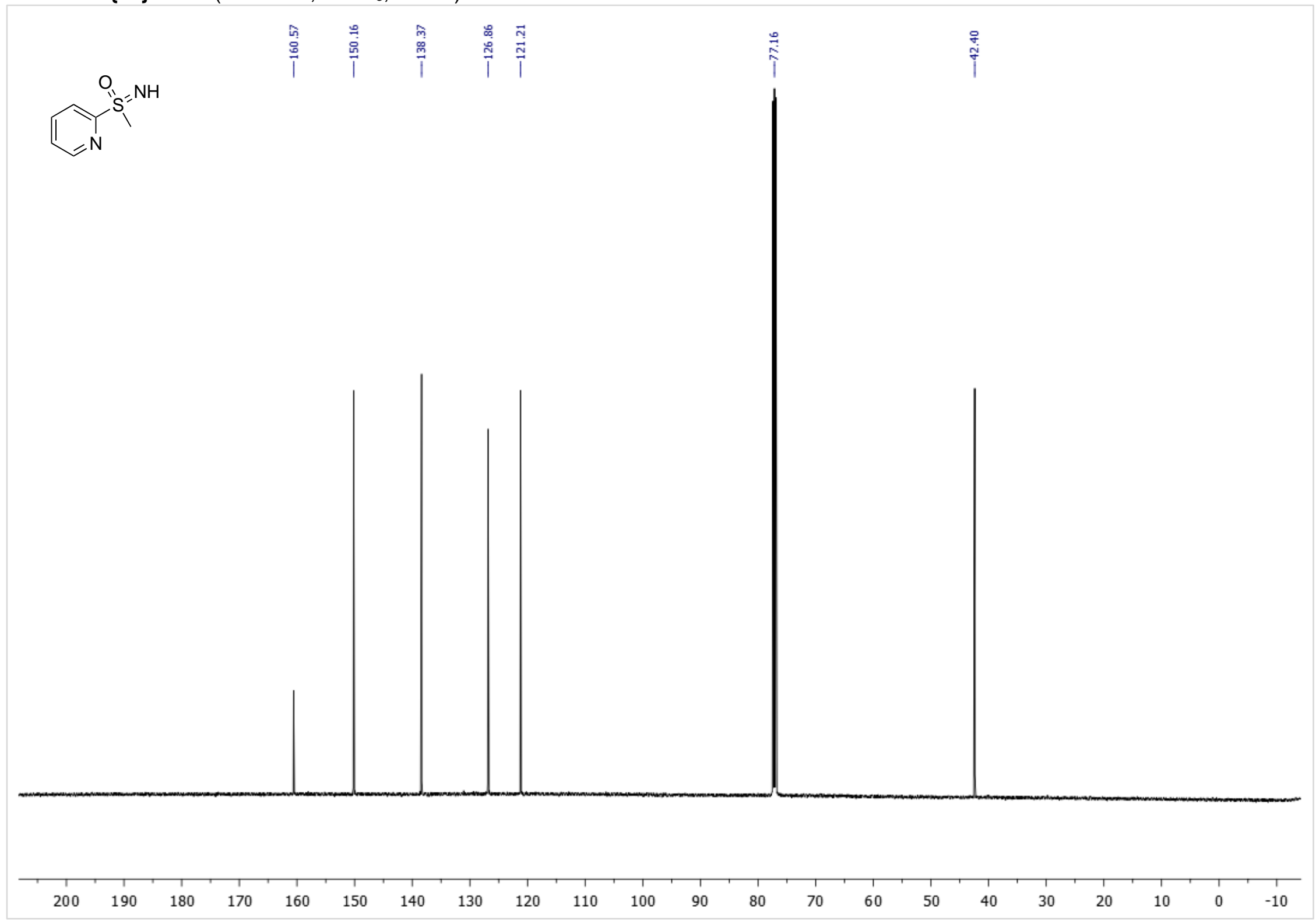

S159 